\title{
Covalent [2]catenane and [2]rotaxane synthesis via a $\delta$-amino acid template
}

Simone Pilon, Steen Ingemann Jørgensen and Jan H. van Maarseveen*

Van't Hoff Institute for Molecular Sciences, University of Amsterdam 1098 XH Amsterdam, The Netherlands e-mail: j.h.vanmaarseveen@uva.nl

\section{Supporting Information}




\section{Table of contents}

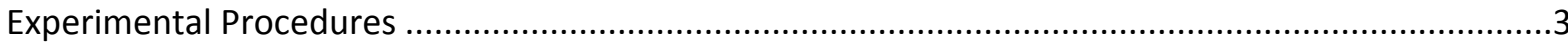

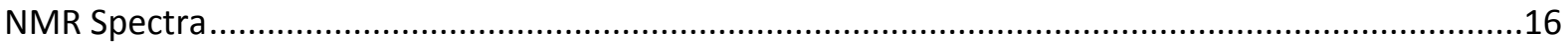

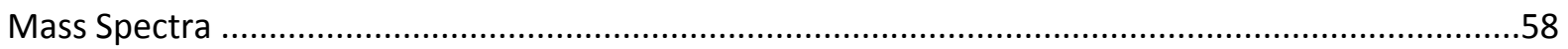

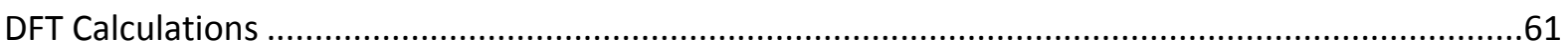

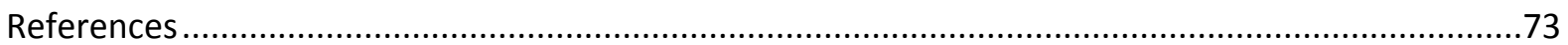




\section{Experimental Procedures}

\section{General methods and materials}

Reactions were carried out under air and without additional measures such as drying unless stated otherwise. Heating and stirring was performed using oil baths and standard thermostatized stirring plates and Teflon stirring beans. Thin layer chromatography (TLC) was performed on Merck TLC plates $(0.25 \mathrm{~mm})$ precoated with silica gel 60 F254. Flash column chromatography was performed using Macherey-Nagel Silica 60 (particle size 0.04-0.063 mm) under compressed air flow or a Buchi C-850 automatic column machine with FlashPure silica cartridges. Where PE is noted as an eluent, $40-60^{\circ} \mathrm{C}$ petroleum ether was used. Starting materials and reagents were used as supplied by commercial vendors. Anhydrous $\mathrm{CH}_{2} \mathrm{Cl}_{2}, \mathrm{MeCN}$ and THF were obtained from pre-dried materials via an MBRAUN SPS-800 machine and stored under $\mathrm{N}_{2}$ atmosphere, anhydrous $\mathrm{MeOH}$ was dried over $3 \AA \AA$ molecular sieves (MS) for 20 hours and stored over fresh $3 \AA \AA \mathrm{MS}$ under a $\mathrm{N}_{2}$ atmosphere. MS were dried at $250^{\circ} \mathrm{C}$ in vacuo for 8 hours before use and stored under $\mathrm{N}_{2}$ atmosphere. Bruker DRX-300, 400 and $500 \mathrm{MHz}$ instruments were used to record NMR spectra. Chemical shifts $(\delta)$ are reported in ppm relative to residual un-deuterated solvent peaks. Data of the recorded ${ }^{1} \mathrm{H}$ NMR spectra are described as follows: chemical shift (multiplicity, coupling constant when applicable, number of $\mathrm{H}$ ). The following abbreviations are used to report the multiplicities: $\mathrm{s}$ (singlet), $\mathrm{d}$ (doublet), $\mathrm{t}$ (triplet), $\mathrm{q}$ (quartet), quint (quintet), dd (doublet of doublet), $m$ (multiplet). High-resolution mass spectra (HRMS) were recorded on an AccuTOF GC v 4g, JMST100GCV mass spectrometer (JEOL, Japan) and HR-ToF Bruker Daltonik GmbH (Bremen, Germany) Impact II, an ESI-ToF MS capable of resolution of at least 40,000 FW HM. The FD/FI probe was equipped with an FD Emitter, Carbotec, FD $=10 \mu \mathrm{m}$. Current rate $=51.2 \mathrm{~mA} / \mathrm{min}$ over $1.2 \mathrm{~min}$ using field desorption (FD) as an ionization method. IR spectra were recorded on a Bruker Alpha FTIR apparatus.

\section{Azido ester 14:}<smiles>CC(=O)c1cc(CN)cc(C(C)=O)c1</smiles>

Trimesic acid (16.0 g, $76.1 \mathrm{mmol}, 1.0$ equiv.) was dissolved in $280 \mathrm{~mL}$ of $\mathrm{MeOH}$, to which $4 \mathrm{~mL}$ $\mathrm{H}_{2} \mathrm{SO}_{4}$ was added dropwise under stirring. The resulting mixture was refluxed for 16 hours, then cooled to $0^{\circ} \mathrm{C}$. The solid residue was obtained by filtration and dried under reduced pressure to remove $\mathrm{MeOH}$. The crude was then dissolved in $40 \mathrm{~mL} \mathrm{CH} \mathrm{Cl}_{2}$, washed $2 \times 20 \mathrm{~mL}$ aqueous saturated $\mathrm{NaHCO}_{3}$ solution, dried over $\mathrm{MgSO}_{4}$ and concentrated in vacuo to give trimethyl benzene-1,3,5-tricarboxylate $(16.8 \mathrm{~g}, 88 \%)$, which was used without further purification. ${ }^{1} \mathrm{H}$ NMR data matched those reported in literature. ${ }^{1}$

Trimethyl benzene-1,3,5-tricarboxylate $(8.00 \mathrm{~g}, 31.7 \mathrm{mmol}, 1.0$ equiv.) was suspended in $700 \mathrm{~mL}$ of $\mathrm{MeOH}$, to which 1.0M aqueous $\mathrm{NaOH}(28.5 \mathrm{~mL}, 28.5 \mathrm{mmol}, 0.9$ equiv.) was added dropwise. After stirring for 16 hours at room temperature, the crude mixture was concentrated in vacuo and the solid residue was partitioned between $100 \mathrm{~mL}$ $\mathrm{CH}_{2} \mathrm{Cl}_{2}$ and $100 \mathrm{~mL}$ aqueous saturated $\mathrm{NaHCO}_{3}$ solution. The organic layer was extracted $3 \times 100 \mathrm{~mL}$ aqueous saturated $\mathrm{NaHCO}_{3}$ and the combined aqueous layers were acidified to ca. $\mathrm{pH} 2$ with $32 \% \mathrm{HCl}$. The resulting precipitate was filtered and rinsed 3 times with AcOEt to give 3,5-bis(methoxycarbonyl)benzoic acid (5.79 g, 76\%), which was used without further purification. ${ }^{1} \mathrm{H}$ NMR data matched those reported in literature. ${ }^{1}$

3,5-bis(methoxycarbonyl)benzoic acid $(5.79 \mathrm{~g}, 24.3 \mathrm{mmol}, 1.0$ equiv.) was dissolved in $60 \mathrm{~mL}$ of anhydrous THF under a $\mathrm{N}_{2}$ atmosphere and a solution of $\mathrm{BH}_{3} \cdot \mathrm{Me}_{2} \mathrm{~S}(4.6 \mathrm{~mL}, 48.6 \mathrm{mmol}, 2.0$ equiv.) in $24 \mathrm{~mL}$ of THF was added dropwise. After stirring the resulting mixture for 16 hours at room temperature, $85 \mathrm{~mL}$ of MeOH were added and the mixture was stirred for an additional hour. The crude mixture was then concentrated in vacuo, diluted in 120 $\mathrm{mL}$ of AcOEt and washed with $100 \mathrm{~mL}$ of $\mathrm{H}_{2} \mathrm{O}, 100 \mathrm{~mL}$ of saturated aqueous $\mathrm{NaHCO}_{3}$ and $100 \mathrm{~mL}$ of brine, dried over $\mathrm{MgSO}_{4}$ and concentrated in vacuo to give dimethyl 5-(hydroxymethyl)isophthalate $(4.22 \mathrm{~g}, 77 \%)$, which was used without further purification. ${ }^{1} \mathrm{H}$ NMR data matched those reported in literature. ${ }^{1}$

Dimethyl 5-(hydroxymethyl)isophthalate ( $2.22 \mathrm{~g}, 9.89 \mathrm{mmol}, 1.0$ equiv.) was dissolved in $\mathrm{SOCl}_{2}(1.44 \mathrm{~mL}, 19.8 \mathrm{mmol}$, 2.0 equiv.) and the mixture was brought to reflux for 1.5 hours, then diluted in $40 \mathrm{~mL} \mathrm{CHCl}$. The resulting solution was washed with $50 \mathrm{~mL}$ of $1 \mathrm{M} \mathrm{NaOH}$, the aqueous phase was extracted with $20 \mathrm{~mL} \mathrm{CHCl}$. The combined organic layers were then washed with brine, dried over $\mathrm{MgSO}_{4}$ and concentrated in vacuo. The crude chloride product obtained was immediately used in the following step, by first dissolving it in $35 \mathrm{~mL}$ of acetone and $12 \mathrm{~mL}$ of $\mathrm{H}_{2} \mathrm{O}$, then adding $\mathrm{NaN}_{3}(3.99 \mathrm{~g}, 61.3 \mathrm{mmol}, 6.0$ equiv.). After refluxing the mixture for 15 hours, the crude product was diluted in $90 \mathrm{~mL}$ of $\mathrm{CHCl}_{3}$, washed 2 times with brine, dried over $\mathrm{MgSO}_{4}$ and concentrated in vacuo to give azido ester template 14 (2.24 g, 88\%), which was used without further purification. ${ }^{1} \mathrm{H}$ NMR data matched those reported in literature. ${ }^{1}$

\section{2-allyloxy-4-methoxybenzaldehyde 15:}<smiles>COc1ccc(C=O)c(OC)c1</smiles>

2-Hydroxy-4-methoxybenzaldehyde (3.04 g, $20.0 \mathrm{mmol}, 1.0$ equiv.) was dissolved in $40 \mathrm{~mL}$ of anhydrous DMF, to which $\mathrm{K}_{2} \mathrm{CO}_{3}(5.52 \mathrm{~g}, 40.0 \mathrm{mmol}, 2.0$ equiv.) and allyl bromide $(3.50 \mathrm{~mL}$, $40.0 \mathrm{mmol}, 2.0$ equiv.) were added. The resulting suspension was stirred for 24 hours at room 
temperature under a $\mathrm{N}_{2}$ atmosphere, then diluted in $200 \mathrm{~mL}$ of a 4:1 PE/AcOEt mixture, washed $3 \times 100 \mathrm{~mL}$ of $\mathrm{H}_{2} \mathrm{O}$, washed with brine, dried over $\mathrm{MgSO}_{4}$ and concentrated in vacuo to give 2-allyloxy-4-methoxybenzaldehyde 15 (3.65 g, 95\%) as a yellow solid which was used without further purification. ${ }^{1} \mathrm{H}$ NMR data matched those reported in literature. $^{2}$

\section{Secondary amine 16:}<smiles>COc1ccc(CNCc2cc(C(C)=O)cc(C(C)=O)c2)c(OC)c1</smiles>

Azido ester 14 ( $2.24 \mathrm{~g}, 8.99 \mathrm{mmol}, 1.0$ equiv.) was dissolved in $50 \mathrm{~mL}$ of $\mathrm{Et}_{2} \mathrm{O}$, to which $\mathrm{PPh}_{3}$ ( $2.59 \mathrm{~g}, 9.89 \mathrm{mmol}, 1.1$ equiv.) was added in portions. After 20 minutes $\mathrm{H}_{2} \mathrm{O}(178 \mu \mathrm{L}, 9.89 \mathrm{mmol}, 1.1$ equiv.) was added. After stirring for 5 hours at room temperature, the crude mixture was concentrated in vacuo, then redissolved in $30 \mathrm{~mL}$ of $\mathrm{MeOH}$. 2-allyloxy-4-methoxybenzaldehyde 15 (1.90 g, $9.89 \mathrm{mmol}, 1.1$ equiv.) was added and the mixture stirred at $35^{\circ} \mathrm{C}$ for 18 hours. Then the solution was cooled to $0^{\circ} \mathrm{C}$ and $\mathrm{NaBH}_{4}$ ( $749 \mathrm{mg}, 19.8 \mathrm{mmol}, 2.2$ equiv.) was added in portions. After 30 minutes, the mixture was allowed to room temperature and stirred for an additional 2 hours, then concentrated in vacuo and partitioned between $100 \mathrm{~mL}$ of AcOEt and $100 \mathrm{~mL}$ aqueous saturated $\mathrm{NaHCO}_{3}$. The aqueous layer was extracted $2 \times 50 \mathrm{~mL}$ AcOEt and the combined organic layers were dried over $\mathrm{MgSO}_{4}$ and concentrated in vacuo. The crude

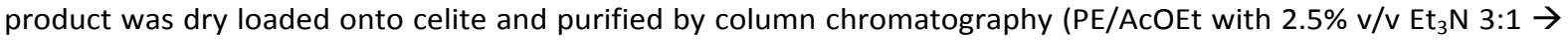
$2: 1)$ to give secondary amine $16(2.99 \mathrm{~g}, 83 \%)$ as a faint yellow oil, which slowly solidifies at room temperature. $R_{\mathrm{f}}=0.34\left(\mathrm{PE} / \mathrm{AcOEt} 1: 1+2.5 \% \mathrm{Et}_{3} \mathrm{~N}\right) ;{ }^{1} \mathrm{H}$ NMR $\left(400 \mathrm{MHz}, \mathrm{CDCl}_{3}\right) \delta 8.55(\mathrm{~s}, 1 \mathrm{H}), 8.19(\mathrm{~d}, \mathrm{~J}=1.7 \mathrm{~Hz}, 2 \mathrm{H}), 7.11(\mathrm{~d}, \mathrm{~J}=8.8$ $\mathrm{Hz}, 1 \mathrm{H}), 6.47-6.39(\mathrm{~m}, 2 \mathrm{H}), 6.00(\mathrm{~m}, 1 \mathrm{H}), 5.36(\mathrm{dd}, \mathrm{J}=17.3,1.6 \mathrm{~Hz}, 1 \mathrm{H}), 5.24(\mathrm{dd}, \mathrm{J}=10.5,1.5 \mathrm{~Hz}, 1 \mathrm{H}), 4.51(\mathrm{~d}, \mathrm{~J}=$ $5.1 \mathrm{~Hz}, 2 \mathrm{H}), 3.92(\mathrm{~s}, 6 \mathrm{H}), 3.83(\mathrm{~s}, 2 \mathrm{H}), 3.77(\mathrm{~s}, 3 \mathrm{H}), 3.76(\mathrm{~s}, 2 \mathrm{H}) ;{ }^{13} \mathrm{C} N M R\left(101 \mathrm{MHz}, \mathrm{CDCl}_{3}\right) \delta$ 166.3, 160.1, 157.6, 141.7, 133.6, 133.1, 130.7, 130.6, 129.3, 120.6, 117.4, 104.1, 99.7, 68.7, 55.4, 52.3, 52.1, 48.4; IR (neat): $v_{\max } / \mathrm{cm}^{-1}$ 2951, 2838, 1722, 1611, 1588, 1505, 1433, 1327, 1289, 1242, 1201, 1164, 1128, 1043, 1004, 928, 830, 786, 755, 721; HRMS (FD') $\mathrm{m} / z$ calcd for $\mathrm{C}_{22} \mathrm{H}_{25} \mathrm{~N}_{1} \mathrm{O}_{6}\left[\mathrm{M}^{\bullet+}\right]$ 399.1676, found 399.1677.

\section{Tertiary amide 17:}

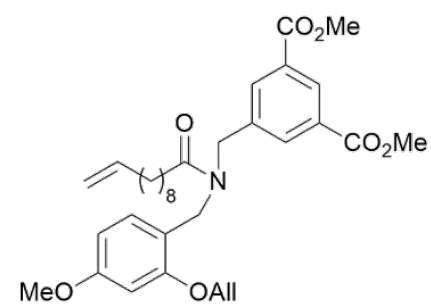

10-undecenoic acid ( $2.76 \mathrm{~g}, 15.0 \mathrm{mmol}, 2.0$ equiv.) was dissolved in $20 \mathrm{~mL} \mathrm{CHCl}$ and $\mathrm{SOCl}_{2}\left(2.20 \mathrm{~mL}, 30.0 \mathrm{mmol}, 4.0\right.$ equiv.) was added to it. After stirring at $60^{\circ} \mathrm{C}$ for 2.5 hours, the crude acyl chloride was concentrated in vacuo, diluted in 30 $\mathrm{mL}$ of anhydrous $\mathrm{CH}_{2} \mathrm{Cl}_{2}$ and added dropwise to a solution of secondary amine 16 (2.99 g, $7.49 \mathrm{mmol}, 1.0$ equiv.) and $\mathrm{Et}_{3} \mathrm{~N}(4.20 \mathrm{~mL}, 30.0 \mathrm{mmol}, 4.0$ equiv.) in $70 \mathrm{~mL}$ of anhydrous $\mathrm{CH}_{2} \mathrm{Cl}_{2}$ kept at $0^{\circ} \mathrm{C}$ under a $\mathrm{N}_{2}$ atmosphere. Once addition was complete, the reaction mixture was allowed to room temperature and stirred for 18 hours, then dry loaded onto silica and purified by column chromatography (PE/AcOEt 3:1 $\rightarrow$ 2:1) to give tertiary amide $17(3.81 \mathrm{~g}, 90 \%)$ as a faint yellow oil as a 7:3 mixture of rotamers.

$R_{\mathrm{f}}=0.37(\mathrm{PE} / \mathrm{AcOEt} 2: 1) ;{ }^{1} \mathrm{H}$ NMR $\left(400 \mathrm{MHz}, \mathrm{CDCl}_{3}\right) \delta 8.59(\mathrm{~s}, 0.3 \mathrm{H}), 8.55(\mathrm{~s}, 0.7 \mathrm{H}), 8.01(\mathrm{~s}, 1.4 \mathrm{H}), 7.97(\mathrm{~s}, 0.6 \mathrm{H}), 7.31$ $(\mathrm{d}, \mathrm{J}=8.4 \mathrm{~Hz}, 0.3 \mathrm{H}), 6.96(\mathrm{~d}, \mathrm{~J}=8.3 \mathrm{~Hz}, 0.7 \mathrm{H}), 6.46(\mathrm{dd}, \mathrm{J}=8.3,2.4 \mathrm{~Hz}, 1 \mathrm{H}), 6.38(\mathrm{dd}, \mathrm{J}=12.6,2.3 \mathrm{~Hz}, 1 \mathrm{H}), 6.07-5.90$ $(\mathrm{m}, 1 \mathrm{H}), 5.90-5.75(\mathrm{~m}, 1 \mathrm{H}), 5.39-5.15(\mathrm{~m}, 2 \mathrm{H}), 5.05-4.90(\mathrm{~m}, 2 \mathrm{H}), 4.67-4.61(\mathrm{~m}, 2 \mathrm{H}), 4.48-4.43(\mathrm{~m}, 3 \mathrm{H}), 4.40$ $(\mathrm{d}, \mathrm{J}=5.2 \mathrm{~Hz}, 1 \mathrm{H}), 3.96(\mathrm{~s}, 1.8 \mathrm{H}), 3.94(\mathrm{~s}, 4.2 \mathrm{H}), 3.80(\mathrm{~s}, 2.1 \mathrm{H}), 3.79(\mathrm{~s}, 0.9 \mathrm{H}), 2.52(\mathrm{t}, \mathrm{J}=7.6 \mathrm{~Hz}, 1.4 \mathrm{H}), 2.33(\mathrm{t}, \mathrm{J}=7.5$ $\mathrm{Hz}, 0.6 \mathrm{H}), 2.10-1.99(\mathrm{~m}, 2 \mathrm{H}), 1.78-1.63(\mathrm{~m}, 2 \mathrm{H}), 1.43-1.23(\mathrm{~m}, 10 \mathrm{H}) ;{ }^{13} \mathrm{C} \mathrm{NMR}\left(101 \mathrm{MHz}, \mathrm{CDCl}_{3}\right) \delta 174.1,173.6$, $166.2,165.9,160.6,160.2,157.5,157.3,139.3,139.2,138.9,133.1,132.8,132.8,131.7,131.5,131.1,130.7,129.7$, 129.4, 128.6, 118.2, 118.2, 117.5, 116.7, 114.1, 104.8, 104.2, 99.6, 99.2, 68.9, 68.8, 55.4, 55.3, 52.4, 52.3, 50.3, 47.8, 46.4, 43.1, 33.8, 33.8, 33.4, 33.2, 29.5, 29.4, 29.3, 29.1, 29.1, 28.9, 28.9, 25.4, 25.3; IR (neat): $v_{\max } / \mathrm{cm}^{-1} 2925,2853$, $1723,1641,1611,1588,1506,1458,1432,1359,1326,1290,1239,1200,1166,1125,1042,1002,926,833,754$, 721; HRMS (FD ${ }^{+}$) $\mathrm{m} / z$ calcd for $\mathrm{C}_{33} \mathrm{H}_{43} \mathrm{~N}_{1} \mathrm{O}_{7}\left[\mathrm{M}^{\bullet+}\right]$ 565.3034, found 565.3060.

Carboxylic acid 18:<smiles>C=CC(=O)N(Cc1cc(C(C)=O)cc(C(=O)O)c1)Cc1ccc(OC)cc1OC</smiles>

Diester 17 ( $2.81 \mathrm{~g}, 4.97 \mathrm{mmol}, 1.0$ equiv.) was dissolved in $100 \mathrm{~mL}$ of $\mathrm{MeOH}$, to which $1.0 \mathrm{M} \mathrm{NaOH}(5.10 \mathrm{~mL}, 5.10 \mathrm{mmol}, 1.05$ equiv.) was added dropwise. The resulting mixture was stirred under reflux for 16 hours, then cooled to room temperature and acidified to $\mathrm{pH} 1$ with ca. $10 \mathrm{~mL} 1 \mathrm{M} \mathrm{KHSO}$. The mixture was then partitioned between $200 \mathrm{~mL} \mathrm{H} \mathrm{H}_{2} \mathrm{O}$ and $200 \mathrm{~mL} \mathrm{AcOEt}$. The aqueous layer was extracted $2 \times 100 \mathrm{~mL}$ AcOEt and the combined organic layers were washed with brine, dried over $\mathrm{MgSO}_{4}$ and concentrated in vacuo. The crude product was dry loaded onto silica and purified by column chromatography (PE/AcOEt 2:1 $\rightarrow$ 1:1 
$\rightarrow 1: 2)$ to give carboxylic acid $18(2.21 \mathrm{~g}, 81 \%)$ as a colorless glass as a 3:1 mixture of rotamers, as well as the diester starting material 17 (334 mg, 12\%).

$R_{\mathrm{f}}=0.20(\mathrm{PE} / \mathrm{AcOEt} 1: 1) ;{ }^{1} \mathrm{H} \mathrm{NMR}\left(400 \mathrm{MHz}, \mathrm{CDCl}_{3}\right) \delta 8.65(\mathrm{~s}, 0.25 \mathrm{H}), 8.59(\mathrm{~s}, 0.75 \mathrm{H}), 8.10-7.95(\mathrm{~m}, 2 \mathrm{H}), 7.29(\mathrm{~d}, \mathrm{~J}=$ $8.4 \mathrm{~Hz}, 0.25 \mathrm{H}), 6.96(\mathrm{~d}, \mathrm{~J}=8.3 \mathrm{~Hz}, 0.75 \mathrm{H}), 6.45(\mathrm{dd}, \mathrm{J}=8.3,2.3 \mathrm{~Hz}, 1 \mathrm{H}), 6.40-6.30(\mathrm{~m}, 1 \mathrm{H}), 6.06-5.70(\mathrm{~m}, 2 \mathrm{H}), 5.38$ $-5.13(\mathrm{~m}, 2 \mathrm{H}), 5.02-4.85(\mathrm{~m}, 2 \mathrm{H}), 4.70-4.61(\mathrm{~m}, 2.5 \mathrm{H}), 4.50-4.42(\mathrm{~m}, 3 \mathrm{H}), 4.39(\mathrm{~d}, \mathrm{~J}=5.2 \mathrm{~Hz}, 0.5 \mathrm{H}), 3.95(\mathrm{~s}$, $0.75 \mathrm{H}), 3.92(\mathrm{~s}, 2.25 \mathrm{H}), 3.78(\mathrm{~s}, 2.25 \mathrm{H}), 3.76(\mathrm{~s}, 0.75 \mathrm{H}), 2.55(\mathrm{t}, \mathrm{J}=7.5 \mathrm{~Hz}, 1.5 \mathrm{H}), 2.36(\mathrm{t}, \mathrm{J}=7.6 \mathrm{~Hz}, 0.5 \mathrm{H}), 2.00(\mathrm{p}, \mathrm{J}=$ $6.7 \mathrm{~Hz}, 2 \mathrm{H}), 1.78-1.62(\mathrm{~m}, 2 \mathrm{H}), 1.41-1.18(\mathrm{~m}, 10 \mathrm{H}) ;{ }^{13} \mathrm{C} \mathrm{NMR}\left(101 \mathrm{MHz}, \mathrm{CDCl}_{3}\right) \delta$ 174.6, 174.1, 169.9, 169.6, 166.1, $165.9,160.6,160.3,157.5,157.3,139.2$, 139.2, 138.8, 133.7, 132.8, 132.7, 132.1, 132.1, 131.8, 131.2, 130.8, 130.8, $130.3,130.2,130.0,128.8,118.3,118.0,117.6,116.5,114.1,114.1,104.8,104.3,99.6,99.3,68.9,68.8,55.4,55.3$, $52.5,52.3,50.3,47.9,46.6,43.2,33.8,33.8,33.8,33.5,33.3,31.9,29.7,29.7,29.6,29.5,29.5,29.4,29.4,29.3,29.2$, 29.1, 29.1, 29.0, 28.9, 28.9, 25.4, 22.7, 14.1; IR (neat): $v_{\max } / \mathrm{cm}^{-1} 2925,2853,1723,1640,1610,1588,1507,1461$,

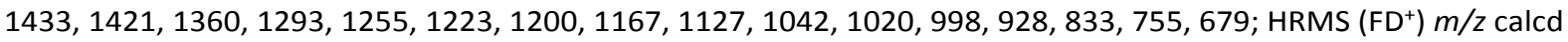
for $\mathrm{C}_{32} \mathrm{H}_{41} \mathrm{~N}_{1} \mathrm{O}_{7}\left[\mathrm{M}^{\bullet+}\right]$ 551.2878, found 551.2886.

Amine 19:<smiles>C=CCNCc1ccc(OC)cc1O</smiles>

10-Undecen-1-amine ${ }^{3}(2.02 \mathrm{~g}, 12.0 \mathrm{mmol}, 1.0$ equiv.) was dissolved in $60 \mathrm{~mL}$ of absolute $\mathrm{MeOH}$, to which 2-allyloxy-4-methoxybenzaldehyde $(2.31 \mathrm{~g}, 12.0 \mathrm{mmol}, 1.0$ equiv.) was added. After stirring at room temperature for 16 hours, the crude mixture was cooled to $0^{\circ} \mathrm{C}$ and $\mathrm{NaBH}_{4}(908 \mathrm{mg}, 24.0 \mathrm{mmol}, 2.0$ equiv.) was added in portions. The resulting mixture was stirred for 1 hour, then warmed to room temperature and stirred for 1 additional hour. Subsequently, the crude was concentrated in vacuo and partitioned between $80 \mathrm{~mL}$ of AcOEt and $80 \mathrm{~mL}$ of a NaHCO saturated aqueous solution. The aqueous layer was extracted $2 \times 40 \mathrm{~mL}$ of AcOEt and the combined organic layers were washed with brine, dried over $\mathrm{MgSO}_{4}$ and concentrated in vacuo to give secondary amine $19(4.07 \mathrm{~g}, 98 \%)$ as a dark amber oil which was used without further purification.

${ }^{1} \mathrm{H}$ NMR $\left(400 \mathrm{MHz}, \mathrm{CDCl}_{3}\right) \delta 7.19-7.12(\mathrm{~m}, 1 \mathrm{H}), 6.48-6.40(\mathrm{~m}, 2 \mathrm{H}), 6.11-5.98(\mathrm{~m}, 1 \mathrm{H}), 5.87-5.73(\mathrm{~m}, 1 \mathrm{H}), 5.41$ $(\mathrm{dd}, \mathrm{J}=17.3,1.7 \mathrm{~Hz}, 1 \mathrm{H}), 5.28(\mathrm{dd}, \mathrm{J}=10.5,1.5 \mathrm{~Hz}, 1 \mathrm{H}), 5.03-4.89(\mathrm{~m}, 2 \mathrm{H}), 4.53(\mathrm{~d}, \mathrm{~J}=5.1 \mathrm{~Hz}, 2 \mathrm{H}), 3.78(\mathrm{~s}, 3 \mathrm{H}), 3.76$ $(\mathrm{s}, 2 \mathrm{H}), 2.58(\mathrm{t}, \mathrm{J}=7.3 \mathrm{~Hz}, 2 \mathrm{H}), 2.08-1.98(\mathrm{~m}, 2 \mathrm{H}), 1.50(\mathrm{t}, \mathrm{J}=7.2 \mathrm{~Hz}, 2 \mathrm{H}), 1.36(\mathrm{t}, \mathrm{J}=7.3 \mathrm{~Hz}, 2 \mathrm{H}), 1.31-1.23(\mathrm{~m}$, $10 \mathrm{H}) ;{ }^{13} \mathrm{C} \mathrm{NMR}\left(101 \mathrm{MHz} \mathrm{CDCl}_{3}\right) \delta 160.0,157.6,139.2,133.2,130.7,120.7,117.3,114.1,104.1,99.7,68.7,55.3$, $48.9,48.8,33.8,29.8,29.5,29.5,29.4,29.1,28.9,27.4$; IR (neat): $v_{\max } / \mathrm{cm}^{-1} 3076,2923,2852,1639,1612,1588$, $1505,1461,1442,1422,1361,1332,1287,1258,1198,1163,1127,1044,1025,996,910,832,788,722,632,568$; HRMS (FD $\left.{ }^{+}\right) \mathrm{m} / z$ calcd for $\mathrm{C}_{22} \mathrm{H}_{35} \mathrm{~N}_{1} \mathrm{O}_{2}\left[\mathrm{M}^{\bullet+}\right]$ 345.2662, found 345.2677.

Bisamide 6:

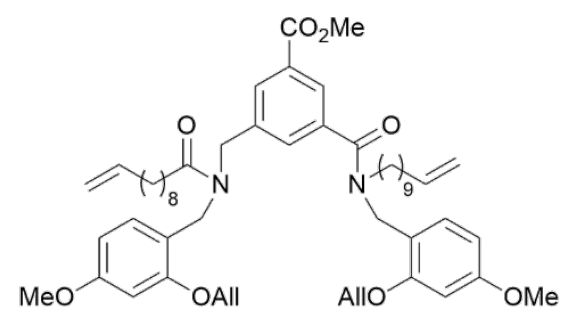

Carboxylic acid 18 (2.21 g, $4.01 \mathrm{mmol}, 1.0$ equiv.) was suspended in $100 \mathrm{~mL}$ of anhydrous $\mathrm{CH}_{2} \mathrm{Cl}_{2}$ under a $\mathrm{N}_{2}$ atmosphere and HOBt (596 $\mathrm{mg}, 4.41 \mathrm{mmol}, 1.1$ equiv.), DMAP ( $49 \mathrm{mg}, 0.40 \mathrm{mmol}, 0.1$ equiv.), DCC ( $2.72 \mathrm{~g}, 13.2 \mathrm{mmol}, 3.3$ equiv.) were added. Finally, amine 19 (1.52 g, $4.41 \mathrm{mmol}, 1.1$ equiv.) in $10 \mathrm{~mL}$ of anhydrous $\mathrm{CH}_{2} \mathrm{Cl}_{2}$ was added and the resulting mixture was stirred at room temperature for 17 hours. The crude mixture was dry loaded onto silica and purified by column chromatography (PE/AcOEt 3:1 $\rightarrow 2: 1 \rightarrow 1: 1$ ) to give bisamide 6 (3.22 $\mathrm{g}, 91 \%)$ as a colorless oil as a mixture of 4 rotamers.

$R_{\mathrm{f}}=0.64(\mathrm{PE} / \mathrm{AcOEt} 1: 1) ;{ }^{1} \mathrm{H} \mathrm{NMR}\left(400 \mathrm{MHz}, \mathrm{CDCl}_{3}\right) \delta 8.03-7.89(\mathrm{~m}, 1 \mathrm{H}), 7.85-7.72(\mathrm{~m}, 1 \mathrm{H}), 7.42(\mathrm{~s}, 1 \mathrm{H}), 7.38-7.27$ $(\mathrm{m}, 0.75 \mathrm{H}), 7.25-7.16(\mathrm{~m}, 0.25 \mathrm{H}), 6.99(\mathrm{~d}, \mathrm{~J}=8.2 \mathrm{~Hz}, 0.5 \mathrm{H}), 6.95-6.82(\mathrm{~m}, 0.5 \mathrm{H}), 6.55-6.31(\mathrm{~m}, 4 \mathrm{H}), 6.12-5.70$ $(\mathrm{m}, 4 \mathrm{H}), 5.48-5.09(\mathrm{~m}, 4 \mathrm{H}), 5.04-4.86(\mathrm{~m}, 4 \mathrm{H}), 4.74(\mathrm{~s}, 1 \mathrm{H}), 4.65-4.30(\mathrm{~m}, 9 \mathrm{H}), 3.96-3.84(\mathrm{~m}, 3 \mathrm{H}), 3.83-3.70$ $(\mathrm{m}, 6 \mathrm{H}), 3.54-3.31(\mathrm{~m}, 1.25 \mathrm{H}), 3.11-3.00(\mathrm{~m}, 0.75 \mathrm{H}), 2.51-2.39(\mathrm{~m}, 1.25 \mathrm{H}), 2.33-2.17(\mathrm{~m}, 0.75 \mathrm{H}), 2.10-1.96$ $(\mathrm{m}, 3 \mathrm{H}), 1.96-1.85(\mathrm{~m}, 1 \mathrm{H}), 1.75-1.53(\mathrm{~m}, 3 \mathrm{H}), 1.47(\mathrm{~s}, 1 \mathrm{H}), 1.42-0.96(\mathrm{~m}, 22 \mathrm{H}) ;{ }^{13} \mathrm{C} \mathrm{NMR}\left(101 \mathrm{MHz}, \mathrm{CDCl}_{3}\right) \delta$ $174.1,173.4,170.7,166.3,166.1,160.5,160.4,160.2,157.6,157.5,157.3,157.2,156.9,139.3,139.2,139.2,139.1$, 139.0, 138.8, 137.7, 133.1, 132.9, 132.9, 132.8, 131.4, 130.8, 130.5, 130.3, 129.5, 129.1, 128.6, 128.6, 128.3, 128.3, $128.1,126.9,126.5,118.2,118.1,118.0,117.8,117.6,117.5,117.3,117.0,116.7,114.1,104.7,104.3,104.2,99.8$, $99.7,99.6,99.5,99.3,69.0,68.9,68.8,68.7,55.4,55.4,55.3,52.3,52.2,52.2,50.2,49.0,48.3,47.8,47.6,46.0,45.0$, $44.7,43.1,41.6,33.9,33.8,33.8,33.4,33.1,31.9,29.7,29.7,29.6,29.5,29.5,29.4,29.4,29.3,29.2,29.1,29.1,28.9$, 28.3, 27.2, 27.1, 26.6, 25.6, 25.4, 24.9, 22.7, 14.1; IR (neat): $v_{\max } / \mathrm{cm}^{-1} 2924,2852,1725,1637,1612,1588,1506$,

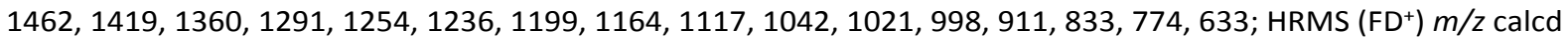
for $\mathrm{C}_{54} \mathrm{H}_{74} \mathrm{~N}_{2} \mathrm{O}_{8}\left[\mathrm{M}^{\bullet+}\right]$ 878.5440, found 878.5433. 
<smiles>C=CNC(=O)N(Cc1cc(C(=O)OC)cc(C(=O)OC)c1)Cc1ccc(OC)cc1OCC</smiles>

Methyl ester 6 ( $1.71 \mathrm{~g}, 1.95 \mathrm{mmol}, 1.0$ equiv.) was dissolved in $200 \mathrm{~mL}$ of a $\mathrm{THF} / \mathrm{H}_{2} \mathrm{O} / \mathrm{MeOH}$ 2:2:1 mixture, to which a $1 \mathrm{M} \mathrm{NaOH}(19.5 \mathrm{~mL}$, $19.5 \mathrm{mmol}, 10$ equiv.) solution was added. After stirring at room temperature for 17 hours, the crude mixture was concentrated in vacuo to remove most of the THF and $\mathrm{MeOH}$ and subsequently acidified to $\mathrm{pH} 1$ with $1 \mathrm{M} \mathrm{KHSO}_{4}$. The resulting mixture was extracted with $3 \times 125 \mathrm{~mL}$ EtOAc, then the combined organic layers were washed with brine, dried over $\mathrm{MgSO}_{4}$ and concentrated in vacuo. The crude carboxylic acid was dissolved in $60 \mathrm{~mL}$ of anhydrous $\mathrm{CH}_{2} \mathrm{Cl}_{2}$ under a $\mathrm{N}_{2}$ atmosphere and pentafluorophenol (718 mg, $3.90 \mathrm{mmol}, 2.0$ equiv.), DiPEA (1.02 mL, $5.85 \mathrm{mmol}, 3.0$ equiv.) and HBTU (1.11 g, $2.93 \mathrm{mmol}, 1.5$ equiv.) were added. The resulting mixture was stirred for 23 hours at room temperature and subsequently dry loaded onto silica and purified by column chromatography (PE/EtOAc 4:1 $\rightarrow$ 3:1 $\rightarrow 2: 1)$ to give pentafluorophenol ester $20(1.87 \mathrm{~g}, 93 \%)$ as a faint yellow oil.

$R_{\mathrm{f}}=0.29$ (PE/AcOEt 3:1); ${ }^{1} \mathrm{H}$ NMR $\left(400 \mathrm{MHz}, \mathrm{CDCl}_{3}\right) \delta 8.19-8.05(\mathrm{~m}, 1 \mathrm{H}), 7.98-7.87(\mathrm{~m}, 1 \mathrm{H}), 7.62-7.46(\mathrm{~m}, 1 \mathrm{H})$, $7.39-7.21(\mathrm{~m}, 1 \mathrm{H}), 7.08-6.88(\mathrm{~m}, 1 \mathrm{H}), 6.59-6.33(\mathrm{~m}, 4 \mathrm{H}), 6.15-5.73(\mathrm{~m}, 4 \mathrm{H}), 5.50-5.14(\mathrm{~m}, 4 \mathrm{H}), 5.06-4.89$ $(\mathrm{m}, 4 \mathrm{H}), 4.79(\mathrm{~s}, 1 \mathrm{H}), 4.71-4.33(\mathrm{~m}, 9 \mathrm{H}), 3.87-3.72(\mathrm{~m}, 6 \mathrm{H}), 3.52-3.39(\mathrm{~m}, 1 \mathrm{H}), 3.16-3.05(\mathrm{~m}, 1 \mathrm{H}), 2.59-2.42$ $(\mathrm{m}, 2 \mathrm{H}), 2.11-1.97(\mathrm{~m}, 4 \mathrm{H}), 1.83-1.47(\mathrm{~m}, 4 \mathrm{H}), 1.45-1.01(\mathrm{~m}, 22 \mathrm{H}) ;{ }^{13} \mathrm{C} \mathrm{NMR}\left(101 \mathrm{MHz}, \mathrm{CDCl}_{3}\right) \delta 174.2,170.1$, $161.9,160.6,160.5,160.3,157.5,157.4,157.2,142.6,139.9,139.7,139.2,139.2,138.1,136.7,133.0,132.8,132.8$, $132.7,132.2,131.0,130.5,129.0,128.6,128.6,127.9,127.1,118.3,118.0,117.9,117.8,117.7,117.1,116.5,114.1$, 104.8, 104.3, 99.7, 99.6, 99.5, 69.0, 68.9, 68.8, 55.4, 55.3, 50.2, 48.4, 48.1, 47.8, 46.4, 45.1, 41.7, 33.8, 33.8, 33.2, 29.5, 29.5, 29.4, 29.4, 29.3, 29.2, 29.1, 29.1, 28.9, 28.9, 28.4, 27.2, 27.1, 26.6, 25.4; IR (neat): $v_{\max } / \mathrm{cm}^{-1} 3077,2925$, 2853, 1761, 1637, 1612, 1588, 1519, 1507, 1463, 1419, 1360, 1291, 1257, 1222, 1199, 1165, 1124, 1077, 1041, 994, $911,833,788,744,628,556$; $\mathrm{HRMS}\left(\mathrm{FD}^{+}\right) \mathrm{m} / z$ calcd for $\mathrm{C}_{59} \mathrm{H}_{71} \mathrm{~F}_{5} \mathrm{~N}_{2} \mathrm{O}_{8}\left[\mathrm{M}^{\bullet+}\right]$ 1030.5125, found 1030.5108 .

\section{dibenzyl alcohol 21:}

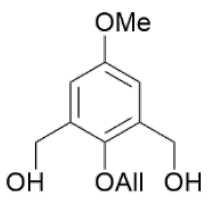

2-Hydroxy-5-methoxy-1,3-benzenedimethanol ${ }^{4}(10.5 \mathrm{~g}, 57.1 \mathrm{mmol}, 1.0$ equiv.) was dissolved in $80 \mathrm{~mL}$ of anhydrous DMF under a $\mathrm{N}_{2}$ atmosphere. $\mathrm{K}_{2} \mathrm{CO}_{3}(20.7 \mathrm{~g}, 150 \mathrm{mmol}, 3.0$ equiv.) was added, followed by dropwise addition of Allyl bromide $(4.9 \mathrm{~mL}, 57.1 \mathrm{mmol}, 1.0$ equiv.) in $15 \mathrm{~mL}$ anhydrous DMF. After stirring for 24 hours at room temperature, the mixture was slowly poured into $150 \mathrm{~mL} \mathrm{H} \mathrm{H}_{2}$ and extracted with $3 \times 100 \mathrm{~mL}$ EtOAc. The combined organic layers were washed with $2 \times 100 \mathrm{~mL}$ aqueous saturated $\mathrm{NH}_{4} \mathrm{Cl}, 2 \times 100 \mathrm{~mL} \mathrm{H}_{2} \mathrm{O}$, brine, dried over $\mathrm{MgSO}_{4}$ and concentrated in vacuo to give dibenzyl alcohol 21 (10.4 g, 81\%) which was used without further purification. ${ }^{1} \mathrm{H}$ NMR $\left(300 \mathrm{MHz}, \mathrm{CDCl}_{3}\right) \delta 6.88(\mathrm{~s}, 2 \mathrm{H}), 6.19-6.00(\mathrm{~m}, 1 \mathrm{H}), 5.42(\mathrm{dd}, \mathrm{J}=17.2,1.6 \mathrm{~Hz}, 1 \mathrm{H}), 5.29(\mathrm{dd}, \mathrm{J}=10.4,1.4$ $\mathrm{Hz}, 1 \mathrm{H}), 4.70(\mathrm{~s}, 4 \mathrm{H}), 4.39(\mathrm{dt}, \mathrm{J}=5.6,1.2 \mathrm{~Hz}, 2 \mathrm{H}), 3.80(\mathrm{~s}, 3 \mathrm{H}) ;{ }^{13} \mathrm{C} \mathrm{NMR}\left(101 \mathrm{MHz}, \mathrm{CDCl}_{3}\right) \delta 156.2,148.3,135.2$, 133.6, 117.9, 113.5, 75.7, 61.1, 55.6; IR (neat): $v_{\max } / \mathrm{cm}^{-1} 3264,2917,2873,2831,1478,1453,1414,1350,1308$, $1236,1211,1191,1145,1055,1036,982,948,931,853,812,700,663,617,559,537 ; \mathrm{HRMS}^{(\mathrm{Fl}} \mathrm{I}^{+} \mathrm{m} / \mathrm{z}$ calcd for $\mathrm{C}_{12} \mathrm{H}_{16} \mathrm{O}_{4}\left[\mathrm{M}^{\bullet+}\right] 224.1043$, found 224.1044.

\section{Allyl protected dialkyne 22:}<smiles>C#CCOCc1cc(OC)cc(COC#C)c1OC</smiles>

Dibenzyl alcohol 21 (1.12 g, $5.00 \mathrm{mmol}, 1.0$ equiv.) was dissolved in $40 \mathrm{~mL}$ of anhydrous $\mathrm{CH}_{2} \mathrm{Cl}_{2}$ and cooled to $-10^{\circ} \mathrm{C}$ under a $\mathrm{N}_{2}$ atmosphere. Then $\mathrm{Et}_{3} \mathrm{~N}(1.7$ $\mathrm{mL}, 12 \mathrm{mmol}, 2.4$ equiv.) was added, followed by dropwise addition of $\mathrm{MsCl}$ $\left(0.85 \mathrm{~mL}, 11.0 \mathrm{mmol}, 2.2\right.$ equiv.). After stirring at $-10^{\circ} \mathrm{C}$ for 3 hours, the reaction was quenched with $20 \mathrm{~mL}$ of $\mathrm{H}_{2} \mathrm{O}$. The aqueous layer was then separated and extracted $2 \times 20 \mathrm{~mL} \mathrm{CH} \mathrm{Cl}_{2}$ and the combined organic layers were washed with $2 \times 20 \mathrm{~mL} \mathrm{H}_{2} \mathrm{O}$, brine, dried over $\mathrm{MgSO}_{4}$ and concentrated in vacuo at room temperature. This intermediate mesylate product is unstable and can degrade upon storage, so it was used immediately.

4-pentyn-1-ol (1.34 g, $16.5 \mathrm{mmol}, 3.3$ equiv.) was dissolved in $10 \mathrm{~mL}$ of anhydrous THF under a $\mathrm{N}_{2}$ atmosphere and cooled to $0^{\circ} \mathrm{C}$. Then $60 \% \mathrm{NaH}$ in mineral oil $(0.600 \mathrm{~g}, 15.0 \mathrm{mmol}, 3$ equiv.) was added in portions and the resulting suspension was stirred for 30 minutes before being added dropwise to a solution of crude mesylate product in 30 $\mathrm{mL}$ of anhydrous THF kept at $0^{\circ} \mathrm{C}$. Once addition was complete, the reaction mixture was heated to reflux for 2 hours and then cooled to room temperature. The crude mixture was poured into $100 \mathrm{~mL}$ of saturated aqueous $\mathrm{NH}_{4} \mathrm{Cl}$ and extracted $3 \times 20 \mathrm{~mL}$ EtOAc. The combined organic layers were washed with brine, dried over $\mathrm{MgSO}_{4}$ and concentrated in vacuo. The crude product was dry loaded onto silica and purified by column chromatography (PE/EtOAc 9:1) to give allyl protected dialkyne $22(1.47 \mathrm{~g}, 83 \%)$ as a colorless oil.

$R_{\mathrm{f}}=0.44$ (PE/AcOEt 8:2); ${ }^{1} \mathrm{H} \mathrm{NMR}\left(400 \mathrm{MHz}, \mathrm{CDCl}_{3}\right) \delta 6.89(\mathrm{~s}, 2 \mathrm{H}), 6.06(\mathrm{~d}, \mathrm{~J}=17.2 \mathrm{~Hz}, 1 \mathrm{H}), 5.41(\mathrm{dd}, \mathrm{J}=17.2,1.7 \mathrm{~Hz}$, $1 \mathrm{H}), 5.25(\mathrm{dd}, \mathrm{J}=10.5,1.5 \mathrm{~Hz}, 1 \mathrm{H}), 4.52(\mathrm{~s}, 4 \mathrm{H}), 4.32(\mathrm{~d}, \mathrm{~J}=5.5 \mathrm{~Hz}, 2 \mathrm{H}), 3.79(\mathrm{~s}, 3 \mathrm{H}), 3.59(\mathrm{t}, \mathrm{J}=6.2 \mathrm{~Hz}, 4 \mathrm{H}), 2.31(\mathrm{td}$, 
$\mathrm{J}=7.1,2.7 \mathrm{~Hz}, 4 \mathrm{H}), 1.94(\mathrm{t}, \mathrm{J}=2.7 \mathrm{~Hz}, 2 \mathrm{H}), 1.83(\mathrm{p}, \mathrm{J}=6.6 \mathrm{~Hz}, 4 \mathrm{H}) ;{ }^{13} \mathrm{C} \mathrm{NMR}\left(101 \mathrm{MHz}, \mathrm{CDCl}_{3}\right) \delta$ 156.0, 148.7, 133.9, 132.6, 117.1, 114.0, 83.9, 75.9, 69.0, 68.6, 67.9, 55.5, 28.7, 15.3; IR (neat): $v_{\max } / \mathrm{cm}^{-1} 3292,2935,2863,1606,1468$, $1440,1409,1361,1321,1287,1248,1198,1152,1100,1056,987,930,858,805,635,558 ; \mathrm{HRMS}^{\left(F D^{+}\right)} \mathrm{m} / \mathrm{z}$ calcd for $\mathrm{C}_{22} \mathrm{H}_{28} \mathrm{O}_{4}\left[\mathrm{M}^{\bullet+}\right]$ 356.1982, found 356.1993.

\section{Dialkyne ring fragment 5:}<smiles>C#COCc1cc(OC)cc(CO[As]C#C)c1O</smiles>

Allyl protected dialkyne 20 ( $1.40 \mathrm{~g}, 3.92 \mathrm{mmol}, 1.0$ equiv.) was dissolved in 20 $\mathrm{mL}$ of absolute $\mathrm{MeOH}$ under a $\mathrm{N}_{2}$ atmosphere, then $\mathrm{K}_{2} \mathrm{CO}_{3}(1.51 \mathrm{~g}, 11.0 \mathrm{mmol}$, 3.0 equiv.) was added. The resulting suspension was sparged with $\mathrm{N}_{2}$ for 15 minutes, then $\mathrm{Pd}\left(\mathrm{PPh}_{3}\right)_{4}(42.2 \mathrm{mg}, 36.5 \mu \mathrm{mol}, 1.0 \mathrm{~mol} \%)$ was added. After stirring at room temperature for 15 hours, $1 \mathrm{M} \mathrm{KHSO}_{4}$ was added until a $\mathrm{pH}$ of ca. 6 was achieved, then $200 \mathrm{~mL}$ of $\mathrm{H}_{2} \mathrm{O}$ was added and the resulting mixture was extracted $3 \times 40 \mathrm{~mL}$ EtOAc. The combined organic layers were then washed with brine, dried over $\mathrm{MgSO}_{4}$ and concentrated in vacuo. The crude product was dry loaded onto silica and purified by column chromatography (PE/EtOAc 19:1 $\rightarrow$ 9:1 $\rightarrow$ 8:2) to give dialkyne ring fragment $5(1.09 \mathrm{~g}, 88 \%)$ as a colorless oil.

$R_{\mathrm{f}}=0.59(\mathrm{PE} / \mathrm{AcOEt} 7: 3) ;{ }^{1} \mathrm{H} \mathrm{NMR}\left(400 \mathrm{MHz}, \mathrm{CDCl}_{3}\right) \delta 7.30(\mathrm{~s}, 1 \mathrm{H}), 6.71(\mathrm{~s}, 2 \mathrm{H}), 4.61(\mathrm{~s}, 4 \mathrm{H}), 3.75(\mathrm{~s}, 3 \mathrm{H}), 3.63(\mathrm{t}, \mathrm{J}=$ $6.2 \mathrm{~Hz}, 4 \mathrm{H}), 2.32(\mathrm{td}, \mathrm{J}=7.1,2.7 \mathrm{~Hz}, 4 \mathrm{H}), 1.95(\mathrm{t}, \mathrm{J}=2.7 \mathrm{~Hz}, 2 \mathrm{H}), 1.91-1.79(\mathrm{~m}, 4 \mathrm{H}) ;{ }^{13} \mathrm{C} \mathrm{NMR}\left(101 \mathrm{MHz}, \mathrm{CDCl}_{3}\right) \delta$ $152.7,147.6,124.7,113.3,83.6,70.1,69.1,68.7,55.8,28.5,15.3$; IR (neat): $v_{\max } / \mathrm{cm}^{-1} 3384,3290,2929,2865,1611$, $1483,1440,1366,1323,1222,1192,1151,1098,1078,1056,944,860,786,638$; HRMS (ESI $) ~ m / z$ calcd for $\mathrm{C}_{19} \mathrm{H}_{23} \mathrm{O}_{4}$ [M-H] 315.1602 , found 315.1605 .

Ester 23:

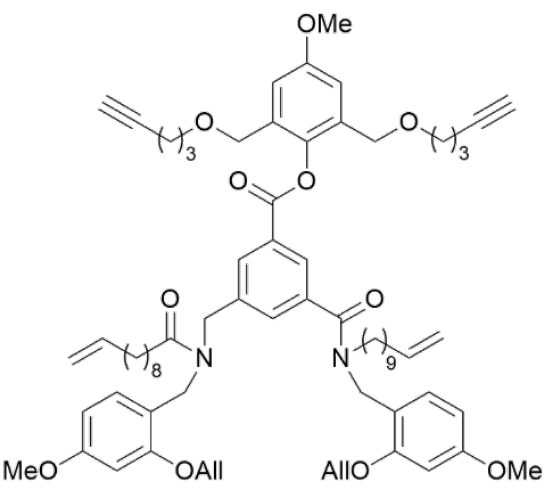

Pentafluorophenol ester 20 (1.87 g, $1.81 \mathrm{mmol}, 1.0$ equiv.) was dissolved in $90 \mathrm{~mL}$ of anhydrous $\mathrm{MeCN}$ under a $\mathrm{N}_{2}$ atmosphere, to which phenol 7 (860 mg, $2.72 \mathrm{mmol}, 1.5$ equiv.), $\mathrm{Cs}_{2} \mathrm{CO}_{3}$ (3.54 g, 10.9 mmol, 6.0 equiv.) and $4.5 \mathrm{~g}$ of $4 \AA$ molecular sieves were added. After stirring for 21 hours at room temperature, the suspension was filtered over celite with some EtOAc and the filtrate was dry loaded onto silica and purified by column chromatography (PE/EtOAc 4:1 $\rightarrow 3: 1 \rightarrow 2: 1$ ) to give ester $23(1.95 \mathrm{~g}, 93 \%)$ as a faint yellow oil.

$R_{\mathrm{f}}=0.47$ (PE/AcOEt 1:2); ${ }^{1} \mathrm{H}$ NMR $\left(500 \mathrm{MHz}, \mathrm{CDCl}_{3}\right) \delta 8.20-8.04(\mathrm{~m}$, $1 \mathrm{H}), 8.00-7.90(\mathrm{~m}, 1 \mathrm{H}), 7.57-7.43(\mathrm{~m}, 1 \mathrm{H}), 7.35-7.28(\mathrm{~m}, 0.5 \mathrm{H}), 7.23$ $-7.19(\mathrm{~m}, 0.5 \mathrm{H}), 7.08-6.86(\mathrm{~m}, 3 \mathrm{H}), 6.54-6.35(\mathrm{~m}, 4 \mathrm{H}), 6.12-5.86$ $(\mathrm{m}, 2 \mathrm{H}), 5.85-5.72(\mathrm{~m}, 2 \mathrm{H}), 5.47-5.20(\mathrm{~m}, 4 \mathrm{H}), 5.04-4.88(\mathrm{~m}, 4 \mathrm{H})$, $4.77(\mathrm{~s}, 1 \mathrm{H}), 4.69-4.29(\mathrm{~m}, 13 \mathrm{H}), 3.88-3.68(\mathrm{~m}, 9 \mathrm{H}), 3.52-3.33(\mathrm{~m}$, $5 \mathrm{H}), 3.10(\mathrm{~s}, 1 \mathrm{H}), 2.45(\mathrm{~d}, \mathrm{~J}=7.3 \mathrm{~Hz}, 2 \mathrm{H}), 2.21(\mathrm{~s}, 4 \mathrm{H}), 2.02(\mathrm{~d}, \mathrm{~J}=6.7 \mathrm{~Hz}, 4 \mathrm{H}), 1.86(\mathrm{~s}, 2 \mathrm{H}), 1.72(\mathrm{~d}, \mathrm{~J}=6.3 \mathrm{~Hz}, 8 \mathrm{H})$, $1.42-0.98(\mathrm{~m}, 22 \mathrm{H}) ;{ }^{13} \mathrm{C} \mathrm{NMR}\left(126 \mathrm{MHz}, \mathrm{CDCl}_{3}\right) \delta$ 174.3, 170.6, 164.1, 160.5, 160.4, 160.2, 157.7, 157.5, 157.3, 157.2 , 139.6, 139.3, 139.2, 139.2, 138.0, 133.1, 132.9, 132.9, 132.8, 132.2, 132.2, 131.0, 130.9, 130.0, 129.9, 129.3, 129.0, 128.4, 128.3, 127.2, 118.3, 117.9, 117.6, 116.6, 114.1, 113.3, 113.1, 104.7, 104.7, 104.4, 104.2, 99.7, 99.7, $99.5,83.8,83.8,69.0,68.9,68.8,68.5,67.9,67.8,55.6,55.4,55.3,47.9,47.8,46.3,44.9,41.7,33.8,33.8,33.1,29.5$, 29.5, 29.5, 29.4, 29.4, 29.4, 29.3, 29.1, 28.9, 28.9, 28.6, 28.4, 27.1, 27.1, 26.7, 25.4, 25.4, 15.2; IR (neat): $v_{\max } / \mathrm{cm}^{-1}$ $3296,2924,2853,1738,1636,1611,1588,1533,1506,1464,1440,1419,1362,1291,1257,1227,1195,1169$, $1116,1102,1043,1020,997,912,833,788,749,722,633,557$; HRMS (FD+) $\mathrm{m} / z$ calcd for $\mathrm{C}_{72} \mathrm{H}_{94} \mathrm{~N}_{2} \mathrm{O}_{11}\left[\mathrm{M}^{\bullet+}\right]$ 1162.6852 , found 1162.6864 .<smiles>C#C[As]OCc1cc(OC)cc(CO[14C]#C)c1OC(=O)c1cc(CN(Cc2ccc(OC)cc2O)C(=O)C=C)cc(C(=O)N(CC=C)Cc2ccc(OC)cc2O)c1</smiles>

Allyl protected bisphenol 23 ( $1.17 \mathrm{~g}, 1.00 \mathrm{mmol}, 1$ equiv.) was dissolved in $40 \mathrm{~mL}$ of anhydrous $\mathrm{CH}_{2} \mathrm{Cl}_{2}$ under a $\mathrm{N}_{2}$ atmosphere, to which $\mathrm{Pd}\left(\mathrm{PPh}_{3}\right)_{4}(57.8 \mathrm{mg}, 50.0 \mu \mathrm{mol}, 5.0 \mathrm{~mol} \%)$ was added. Subsequently, a solution of $\mathrm{PhSiH}_{3}(260 \mathrm{mg}, 2.41 \mathrm{mmol}, 2.4$ equiv.) in $10 \mathrm{~mL}$ of anhydrous $\mathrm{CH}_{2} \mathrm{Cl}_{2}$ was added dropwise over 30 minutes. After stirring at room temperature for an additional hour, the crude mixture was dry loaded onto silica and purified by column chromatography (PE/EtOAC $4: 1 \rightarrow 3: 1 \rightarrow 2: 1 \rightarrow 1: 1)$ to give bisphenol 7 (1.04 g, 96\%) as a faint yellow oil. Note that the progress of the reaction was monitored by ${ }^{1} \mathrm{H}$ 
NMR, since product and starting material could not be separated on a TLC plate.

$R_{\mathrm{f}}=0.68$ (PE/AcOEt 1:1); ${ }^{1} \mathrm{H} \mathrm{NMR}\left(400 \mathrm{MHz}, \mathrm{CDCl}_{3}\right) \delta 9.65(\mathrm{~s}, 1 \mathrm{H}), 9.54(\mathrm{~s}, 1 \mathrm{H}), 8.20(\mathrm{~s}, 1 \mathrm{H}), 8.07(\mathrm{~s}, 1 \mathrm{H}), 7.52(\mathrm{~s}, 1 \mathrm{H})$, $7.04(\mathrm{~d}, \mathrm{~J}=8.4 \mathrm{~Hz}, 1 \mathrm{H}), 6.97(\mathrm{~s}, 2 \mathrm{H}), 6.84(\mathrm{~d}, \mathrm{~J}=8.4 \mathrm{~Hz}, 1 \mathrm{H}), 6.56(\mathrm{~d}, \mathrm{~J}=2.6 \mathrm{~Hz}, 1 \mathrm{H}), 6.49(\mathrm{~d}, \mathrm{~J}=2.6 \mathrm{~Hz}, 1 \mathrm{H}), 6.43(\mathrm{dd}$, $\mathrm{J}=8.3,2.6 \mathrm{~Hz}, 1 \mathrm{H}), 6.33(\mathrm{dd}, \mathrm{J}=8.3,2.6 \mathrm{~Hz}, 1 \mathrm{H}), 5.86-5.71(\mathrm{~m}, 2 \mathrm{H}), 5.02-4.87(\mathrm{~m}, 4 \mathrm{H}), 4.63(\mathrm{~d}, \mathrm{~J}=20.4 \mathrm{~Hz}, 4 \mathrm{H})$, $4.41(\mathrm{~s}, 6 \mathrm{H}), 3.85(\mathrm{~s}, 3 \mathrm{H}), 3.79(\mathrm{~s}, 3 \mathrm{H}), 3.72(\mathrm{~s}, 3 \mathrm{H}), 3.46(\mathrm{t}, \mathrm{J}=6.1 \mathrm{~Hz}, 4 \mathrm{H}), 3.24(\mathrm{t}, \mathrm{J}=7.9 \mathrm{~Hz}, 2 \mathrm{H}), 2.35(\mathrm{t}, \mathrm{J}=7.5 \mathrm{~Hz}$, $2 \mathrm{H}), 2.20(\mathrm{td}, \mathrm{J}=7.1,2.6 \mathrm{~Hz}, 4 \mathrm{H}), 2.01(\mathrm{q}, \mathrm{J}=6.7 \mathrm{~Hz}, 4 \mathrm{H}), 1.85(\mathrm{t}, \mathrm{J}=2.6 \mathrm{~Hz}, 2 \mathrm{H}), 1.77-1.60(\mathrm{~m}, 8 \mathrm{H}), 1.40-1.12(\mathrm{~m}$, $22 \mathrm{H}) ;{ }^{13} \mathrm{C} \mathrm{NMR}\left(101 \mathrm{MHz}, \mathrm{CDCl}_{3}\right) \delta 176.1,171.9,163.5,161.8,161.7,157.7,157.6,139.9,139.2,139.1,137.8,136.8$, $132.4,132.1,132.0,132.0,130.6,129.9,129.6,128.0,114.2,114.2,113.9,113.7,113.7,106.1,105.8,102.7,83.8$, $68.8,68.6,68.2,55.6,55.3,55.2,50.0,48.8,46.3,45.4,33.8,33.8,33.3,29.5,29.4,29.4,29.3,29.3,29.1,29.1,28.9$, 28.6, 28.3, 26.9, 25.2, 15.2; IR (neat): $v_{\max } / \mathrm{cm}^{-1} 3289,3075,2924,2853,1739,1612,1507,1466,1436,1363,1323$, $1289,1231,1170,1102,1053,1036,997,964,911,849,786,750,724,637 ; \mathrm{HRMS}^{\left(E S{ }^{+}\right.}$) $\mathrm{m} / z$ calcd for $\mathrm{C}_{66} \mathrm{H}_{87} \mathrm{~N}_{2} \mathrm{O}_{11}$ $\left[\mathrm{M}+\mathrm{H}^{+}\right]$1083.6304, found 1083.7099 .

\section{Methyl ester template 24:}<smiles>COc1cc(OC)c(C(C)=O)cc1O[Al]=[W]</smiles>

3-azido-1-methanesulfonate-1-propanol ${ }^{5}(5.77 \mathrm{~g}, 32.2 \mathrm{mmol}, 2.5$ equiv.) was dissolved in $65 \mathrm{~mL}$ of anhydrous DMF under a $\mathrm{N}_{2}$ atmosphere, to which dimethyl 2,5dihydroxyterephthalate ${ }^{6}\left(2.91 \mathrm{~g}, 12.9 \mathrm{mmol}, 1.0\right.$ equiv.) and $\mathrm{K}_{2} \mathrm{CO}_{3}(4.45 \mathrm{~g}, 32.2 \mathrm{mmol}$, 2.5 equiv.) were added. After stirring for 16 hours at $80^{\circ} \mathrm{C}$, the suspension was cooled to room temperature and partitioned between $100 \mathrm{~mL}$ of $\mathrm{H}_{2} \mathrm{O}$ and $100 \mathrm{~mL}$ of EtOAc. The aqueous layer was extracted $2 \times 50 \mathrm{~mL}$ EtOAc and the combined organic layers were washed with $2 \times 50 \mathrm{~mL} \mathrm{H}_{2} \mathrm{O}$, brine, dried over $\mathrm{MgSO}_{4}$ and concentrated in vacuo. The crude product was dry loaded onto silica and purified by column chromatography (PE/EtOAc 4:1) to give methyl ester template 24 (4.30 g, 85\%) as a faint yellow oil.

$R_{\mathrm{f}}=0.24(\mathrm{PE} / \mathrm{AcOEt} 3: 1) ;{ }^{1} \mathrm{H} \mathrm{NMR}\left(300 \mathrm{MHz}, \mathrm{CDCl}_{3}\right) \delta 7.39(\mathrm{~s}, 2 \mathrm{H}), 4.10(\mathrm{t}, \mathrm{J}=5.8 \mathrm{~Hz}, 4 \mathrm{H}), 3.89(\mathrm{~s}, 6 \mathrm{H}), 3.56(\mathrm{t}, \mathrm{J}=6.5$ $\mathrm{Hz}, 4 \mathrm{H}), 2.06(\mathrm{p}, \mathrm{J}=6.2 \mathrm{~Hz}, 4 \mathrm{H}) ;{ }^{13} \mathrm{C} \mathrm{NMR}\left(75 \mathrm{MHz} \mathrm{CDCl}_{3}\right) \delta 165.6,151.7,124.4,116.9,66.3,52.3,48.0,28.8 ; \mathrm{IR}$ (neat): $v_{\max } / \mathrm{cm}^{-1} 2951,2880,2091,1726,1707,1502,1467,1435,1408,1382,1345,1299,1231,1196,1098,1044$, 979, 919, 882, 787, 751, 626, 556; HRMS $\left(\mathrm{FD}^{+}\right) \mathrm{m} / z$ calcd for $\mathrm{C}_{16} \mathrm{H}_{20} \mathrm{~N}_{6} \mathrm{O}_{6}\left[\mathrm{M}^{\bullet+}\right]$ 392.1439, found 392.1453.

\section{Pentafluorophenol ester template 4:}

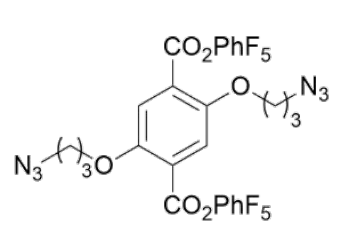

Methyl ester template 24 ( $4.30 \mathrm{~g}, 11.0 \mathrm{mmol}, 1.0$ equiv.) was dissolved in $75 \mathrm{~mL}$ of a 2:1 $\mathrm{THF} / \mathrm{MeOH}$ mixture, to which a solution of $\mathrm{KOH}(2.46 \mathrm{~g}, 43.8 \mathrm{mmol}, 4.0$ equiv.) in $25 \mathrm{~mL}$ $\mathrm{H}_{2} \mathrm{O}$ was added. After stirring for 16 hours at room temperature, most of the volatile solvents were removed in vacuo and the resulting crude mixture was acidified to $\mathrm{pH} 1$ with $1 \mathrm{M} \mathrm{KHSO}_{4}$. The solid precipitate was filtered and washed with $2 \times 20 \mathrm{~mL} \mathrm{H} 2 \mathrm{O}, 10 \mathrm{~mL}$ $\mathrm{EtOH}$, suspended in $20 \mathrm{~mL}$ of toluene and concentrated in vacuo to give the crude diacid

(3.22 g, 80\%) which was used without further purification.

The crude diacid ( $3.22 \mathrm{~g}, 8.84 \mathrm{mmol}, 1$ equiv.) was suspended in $90 \mathrm{~mL}$ of anhydrous $\mathrm{CH}_{2} \mathrm{Cl}_{2}$ under a $\mathrm{N}_{2}$ atmosphere, to which DiPEA ( $6.2 \mathrm{~mL}, 35.4 \mathrm{mmol}, 4$ equiv.), pentafluorophenol ( $4.88 \mathrm{~g}, 26.5 \mathrm{mmol}, 3$ equiv.) and HBTU (10.1 g, $26.5 \mathrm{mmol}, 3$ equiv.) were added. After stirring at room temperature for 16 hours the crude mixture was dry loaded on silica and purified by column chromatography (PE/EtOAC 9:1 $\rightarrow$ 8:2) over a short column to remove baseline impurities. The product was then recrystallized from EtOAc and heptane to give pentafluorophenol ester template 4 (5.10 g, 83\%) as a white solid.

$R_{\mathrm{f}}=0.31$ (PE/AcOEt 9:1); ${ }^{1} \mathrm{H} \mathrm{NMR}\left(400 \mathrm{MHz}, \mathrm{CDCl}_{3}\right) \delta 7.66(\mathrm{~s}, 2 \mathrm{H}), 4.22(\mathrm{t}, \mathrm{J}=5.8 \mathrm{~Hz}, 4 \mathrm{H}), 3.57(\mathrm{t}, \mathrm{J}=6.5 \mathrm{~Hz}, 4 \mathrm{H}), 2.11$ $(p, J=6.2 \mathrm{~Hz}, 4 \mathrm{H}) ;{ }^{13} \mathrm{C} \mathrm{NMR}\left(101 \mathrm{MHz}, \mathrm{CDCl}_{3}\right) \delta 160.5,152.7,122.2,117.2,66.4,47.9,28.7$; IR (neat): $v_{\max } / \mathrm{cm}^{-1} 2100$, $1771,1519,1470,1415,1387,1298,1231,1185,1143,1034,996$; HRMS (FD ${ }^{+}$) $m / z$ calcd for $\mathrm{C}_{26} \mathrm{H}_{14} \mathrm{~F}_{10} \mathrm{~N}_{6} \mathrm{O}_{6}\left[\mathrm{M}^{\bullet+}\right.$ ] 696.0810, found 696.0840 .

Bislactone 8:

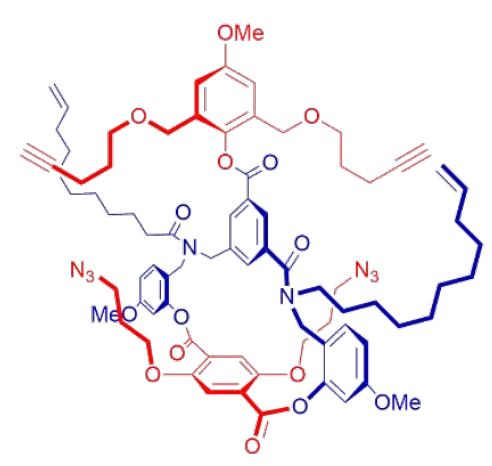

Bisphenol 7 (837 mg, $772 \mu \mathrm{mol}, 1.0$ equiv.) was dissolved in $386 \mathrm{~mL}$ of anhydrous $\mathrm{MeCN}$ under a $\mathrm{N}_{2}$ atmosphere, to which $8.0 \mathrm{~g}$ of $4 \AA \mathrm{AS}$ and $\mathrm{Cs}_{2} \mathrm{CO}_{3}$ ( $2.52 \mathrm{~g}, 7.72 \mathrm{mmol}, 10$ equiv.) were added. Finally, pentafluorophenol ester template 4 ( $565 \mathrm{mg}, 811 \mu \mathrm{mol}, 1.05$ equiv.) was added and the suspension was stirred for 16 hours at $45^{\circ} \mathrm{C}$ before being filtered over celite with some EtOAc. The crude filtrate was dry loaded onto silica and purified by column chromatography (PE/EtOAc 9:1 $\rightarrow$ 8:2 $\rightarrow$ 2:1) to give bislactone 8 (838 mg, $77 \%)$ as a colorless oil.

$R_{\mathrm{f}}=0.34$ (PE/AcOEt 1:1); ${ }^{1} \mathrm{H}$ NMR $\left(400 \mathrm{MHz}, \mathrm{CDCl}_{3}\right) \delta 7.96(\mathrm{~s}, 0.5 \mathrm{H}), 7.88-7.73$ $(\mathrm{m}, 2 \mathrm{H}), 7.65-7.41(\mathrm{~m}, 2 \mathrm{H}), 7.36-7.27(\mathrm{~m}, 2 \mathrm{H}), 7.02-6.77(\mathrm{~m}, 6 \mathrm{H}), 6.42-$ 
$6.23(\mathrm{~m}, 0.5 \mathrm{H}), 5.87-5.69(\mathrm{~m}, 2 \mathrm{H}), 5.04-4.86(\mathrm{~m}, 4 \mathrm{H}), 4.75-4.52(\mathrm{~m}, 1 \mathrm{H}), 4.37(\mathrm{~s}, 4.5 \mathrm{H}), 4.29-4.09(\mathrm{~m}, 4.5 \mathrm{H})$, $3.92-3.76(\mathrm{~m}, 9 \mathrm{H}), 3.64-3.52(\mathrm{~m}, 2 \mathrm{H}), 3.52-3.35(\mathrm{~m}, 6 \mathrm{H}), 3.12-2.82(\mathrm{~m}, 2 \mathrm{H}), 2.42-2.27(\mathrm{~m}, 1.5 \mathrm{H}), 2.26-2.06$ $(\mathrm{m}, 5.5 \mathrm{H}), 2.06-1.91(\mathrm{~m}, 6 \mathrm{H}), 1.90-1.78(\mathrm{~m}, 2 \mathrm{H}), 1.78-1.55(\mathrm{~m}, 7 \mathrm{H}), 1.54-0.93(\mathrm{~m}, 28 \mathrm{H}) ;{ }^{13} \mathrm{C} \mathrm{NMR}(101 \mathrm{MHz}$, $\left.\mathrm{CDCl}_{3}\right) \delta 173.5,169.0,163.7,160.7,159.7,157.7,151.7,150.7,149.6,139.8,139.2,139.1,138.6,138.1,135.1$, 132.7, 132.1, 130.0, 127.0, 121.1, 119.6, 117.8, 115.6, 114.2, 114.1, 113.4, 113.1, 111.9, 111.8, 109.3, 107.7, 83.8, $76.3,68.9,68.8,68.6,68.0,66.3,55.6,55.6,55.6,50.9,48.0,47.8,46.6,44.2,34.0,33.8,33.3,31.4,30.2,29.7,29.5$, 29.4, 29.3, 29.2, 29.1, 29.1, 28.9, 28.9, 28.6, 27.8, 26.5, 25.4, 25.1, 22.7, 15.2, 14.1; IR (neat): $v_{\max } / \mathrm{cm}^{-1} 3295,2925$, 2853, 2096, 1740, 1618, 1504, 1464, 1412, 1388, 1362, 1288, 1254, 1224, 1180, 1151, 1101, 1035, 1010, 950, 912, 874, 747, 633, 556, 465; HRMS $\left(\mathrm{ESI}^{+}\right) \mathrm{m} / \mathrm{z}$ calcd for $\mathrm{C}_{80} \mathrm{H}_{99} \mathrm{~N}_{8} \mathrm{O}_{15}\left[\mathrm{M}+\mathrm{H}^{+}\right]$1411.7224, found 1411.7260.

Triazole macrocycle 1:

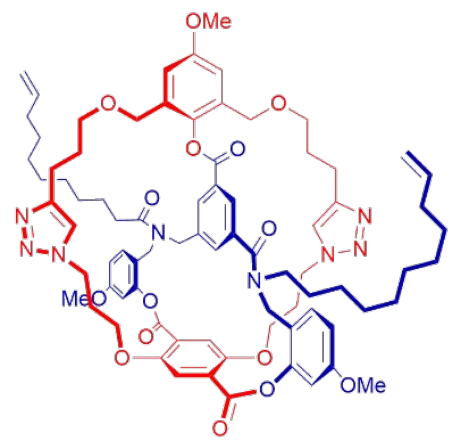

Azidoalkyne 8 ( $838 \mathrm{mg}, 594 \mu \mathrm{mol}, 1.0$ equiv.) was dissolved in $600 \mathrm{~mL}$ of $\mathrm{CH}_{2} \mathrm{Cl}_{2}$ under a $\mathrm{N}_{2}$ atmosphere, to which TBTA (94.6 mg, $178 \mu \mathrm{mol}, 0.3$ equiv.) was added. The resulting solution was sparged with $\mathrm{N}_{2}$ for 1 hour before addition of $\mathrm{Cu}\left(\mathrm{MeCN}_{4}\right) \mathrm{BF}_{4}(56.0 \mathrm{mg}, 178 \mu \mathrm{mol}, 0.3$ equiv.). After heating at reflux for 16 hours, the solvent was removed in vacuo and the crude mixture dry loaded onto silica and purified by column chromatography (ACOEt/PE 2:1 $\rightarrow$ 3:1 $\rightarrow$ 4:1 $\rightarrow$ AcOEt) to give triazole macrocycle 1 (652 mg, 78\%) as a white foam.

$R_{\mathrm{f}}=0.24$ (AcOEt/PE 2:1); ${ }^{1} \mathrm{H}$ NMR $\left(400 \mathrm{MHz}, \mathrm{CDCl}_{3}\right) \delta 8.30-7.72(\mathrm{~m}, 4 \mathrm{H}), 7.72-$ $7.43(\mathrm{~m}, 1.5 \mathrm{H}), 7.39-7.20(\mathrm{~m}, 1.5 \mathrm{H}), 7.15-6.94(\mathrm{~m}, 1 \mathrm{H}), 6.94-6.71(\mathrm{~m}, 5 \mathrm{H})$, $6.61(\mathrm{~s}, 1 \mathrm{H}), 6.11-5.90(\mathrm{~m}, 1 \mathrm{H}), 5.88-5.69(\mathrm{~m}, 2 \mathrm{H}), 5.65-5.25(\mathrm{~m}, 1 \mathrm{H}), 5.05$ $-4.83(\mathrm{~m}, 4 \mathrm{H}), 4.83-3.93(\mathrm{~m}, 14 \mathrm{H}), 3.93-3.69(\mathrm{~m}, 9 \mathrm{H}), 3.67-3.17(\mathrm{~m}, 4.5 \mathrm{H})$, $2.82-2.09(\mathrm{~m}, 10 \mathrm{H}), 2.09-1.90(\mathrm{~m}, 4.5 \mathrm{H}), 1.88-1.54(\mathrm{~m}, 6 \mathrm{H}), 1.41-0.78(\mathrm{~m}$, $28 \mathrm{H}) ;{ }^{13} \mathrm{C} \mathrm{NMR}\left(101 \mathrm{MHz}, \mathrm{CDCl}_{3}\right) \delta 173.5,168.8,165.1,163.3,160.8,159.5,157.2,152.7,151.5,151.1,150.5,150.2$, $149.2,142.6,142.2,142.1,139.3,139.2$, 139.1, 139.0, 137.8, 136.0, 132.8, 132.0, 131.6, 129.5, 124.8, 120.8, 119.5, $115.5,115.2,115.0,114.3,114.2,114.1,114.1,114.1,114.0,112.0,111.4,109.7,108.0,76.4,71.0,70.8,70.5,66.5$, 65.7, 55.7, 55.6, 55.6, 51.6, 46.1, 45.2, 43.3, 34.9, 33.8, 33.8, 33.8, 33.8, 33.2, 31.9, 31.4, 30.2, 29.7, 29.7, 29.6, 29.5, 29.5, 29.4, 29.4, 29.4, 29.3, 29.2, 29.2, 29.1, 29.1, 29.1, 29.0, 29.0, 29.0, 28.9, 28.9, 28.9, 27.9, 26.9, 26.8, 25.5, 25.4, 25.2, 22.7, 22.2, 14.1; IR (neat): $v_{\max } / \mathrm{cm}^{-1} 2922,2852,1741,1632,1506,1465,1414,1256,1194,1105,1051$; HRMS $\left(\mathrm{FD}^{+}\right) \mathrm{m} / \mathrm{z}$ calcd for $\mathrm{C}_{80} \mathrm{H}_{99} \mathrm{~N}_{8} \mathrm{O}_{15}\left[\mathrm{M}+\mathrm{H}^{+}\right]$1411.7224, found 1411.7169 .

Alkene bismacrocycle 25:

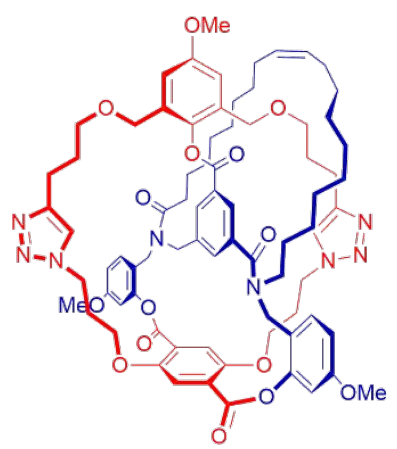

Triazole macrocycle 1 ( $200 \mathrm{mg}, 142 \mu \mathrm{mol}, 1.0$ equiv.) was dissolved in $140 \mathrm{~mL}$ of dichloroethane under a $\mathrm{N}_{2}$ atmosphere. After sparging the solution with $\mathrm{N}_{2}$ for 1 hour, Grubbs $2^{\text {nd }}$ generation catalyst $(48.2 \mathrm{mg}, 56.8 \mu \mathrm{mol}, 0.4$ equiv.) was added and the reaction mixture sparged for an additional 10 minutes. After heating at reflux for 20 hours, the solvents were removed in vacuo and the crude product was purified by column chromatography (EtOAc in $\mathrm{CH}_{2} \mathrm{Cl}_{2} 33 \% \rightarrow 55 \% \rightarrow 80 \%$ ) to give bismacrocycle $25(82.7 \mathrm{mg}, 42 \%)$ as a brown glass, as well as triazole macrocycle starting material 1 (50.1 $\mathrm{mg}, 25 \%)$ which was subjected to identical reaction conditions to give bismacrocycle 25 ( $14.4 \mathrm{mg}, 29 \%)$. Longer reaction times lead to decomposition of the product and overall lower yields.

$R_{\mathrm{f}}=0.18\left(\mathrm{AcOEt} / \mathrm{PE} \mathrm{9:1)} ;{ }^{1} \mathrm{H} \mathrm{NMR}\left(400 \mathrm{MHz}, \mathrm{CDCl}_{3}\right) \delta 8.07(\mathrm{~s}, 0.5 \mathrm{H}), 7.97(\mathrm{~d}, \mathrm{~J}=6.0\right.$ $\mathrm{Hz}, 1 \mathrm{H}), 7.91-7.74(\mathrm{~m}, 2.5 \mathrm{H}), 7.68(\mathrm{~s}, 1 \mathrm{H}), 7.30(\mathrm{~d}, \mathrm{~J}=6.3 \mathrm{~Hz}, 1 \mathrm{H}), 6.93(\mathrm{dd}, \mathrm{J}=6.1$, $2.6 \mathrm{~Hz}, 1 \mathrm{H}), 6.91-6.75(\mathrm{~m}, 5 \mathrm{H}), 6.69(\mathrm{~s}, 0.5 \mathrm{H}), 6.55(\mathrm{~s}, 0.5 \mathrm{H}), 6.20(\mathrm{~s}, 0.5 \mathrm{H}), 5.96(\mathrm{dd}, \mathrm{J}=14.9,8.9 \mathrm{~Hz}, 1 \mathrm{H}), 5.48-$ $5.17(\mathrm{~m}, 2.5 \mathrm{H}), 4.94(\mathrm{~d}, \mathrm{~J}=17.1 \mathrm{~Hz}, 1 \mathrm{H}), 4.85-4.68(\mathrm{~m}, 2 \mathrm{H}), 4.68-4.15(\mathrm{~m}, 11 \mathrm{H}), 3.88(\mathrm{~s}, 3 \mathrm{H}), 3.85-3.79(\mathrm{~m}, 6 \mathrm{H})$, $3.79-3.63(\mathrm{~m}, 1.5 \mathrm{H}), 3.60-3.36(\mathrm{~m}, 3 \mathrm{H}), 3.35-3.21(\mathrm{~m}, 1 \mathrm{H}), 3.08-2.96(\mathrm{~m}, 0.5 \mathrm{H}), 2.77-2.06(\mathrm{~m}, 10 \mathrm{H}), 2.03-$ $1.57(\mathrm{~m}, 10 \mathrm{H}), 1.51-0.61(\mathrm{~m}, 28 \mathrm{H}) ;{ }^{13} \mathrm{C} \mathrm{NMR}\left(101 \mathrm{MHz}, \mathrm{CDCl}_{3}\right) \delta 174.0,174.0,169.1,169.0,168.8,163.7,163.6$, $163.5,163.3,162.2,162.1,162.1,162.0,160.8,159.6,159.6,157.2,157.2,157.1,152.4,152.2,151.7,151.6,151.6$, $150.6,150.5,150.5,149.4,149.4,149.3,147.2,146.9,146.2,146.1,145.5,145.5,142.8,142.7,142.2,140.6,140.0$, $139.9,138.4,138.1,136.0,136.0,132.8,132.7,132.3,132.2,132.2,131.5,131.4,131.2,131.1,130.8,130.5,130.3$, $130.2,130.1,130.0,129.5,128.4,128.3,128.1,127.8,127.6,127.4,125.4,125.2,124.5,124.0,123.9,123.9,121.3$, $121.1,121.1,121.0,120.9,120.8,120.0,119.6,119.4,118.8,116.0,115.9,115.9,115.5,115.2,115.1,111.9,111.5$, 111.4, 109.8, 109.7, 108.1, 108.0, 71.5, 71.4, 71.0, 70.8, 70.6, 70.5, 70.3, 69.8, 66.4, 66.4, 65.9, 65.4, 60.4, 55.7, $55.7,55.6,51.9,51.7,46.4,46.2,46.0,45.8,45.5,45.1,45.0,43.5,43.2,33.3,33.1,32.6,32.6,32.3,32.1,30.6,30.5$, 30.0, 29.9, 29.8, 29.8, 29.7, 29.6, 29.5, 29.5, 29.4, 29.3, 29.3, 29.3, 29.1, 29.0, 28.9, 28.9, 28.8, 28.8, 28.4, 28.3, 28.1, $27.9,27.7,27.4,27.3,27.1,25.8,25.6,25.4,25.2,22.4,22.2,22.1,21.8,21.7,14.2$; IR (neat): $v_{\max } / \mathrm{cm}^{-1} 2924,2853$, 
$1739,1619,1505,1465,1432,1414,1389,1363,1320,1288,1253,1186,1151,1105,1049,966,912,731 ;$ HRMS $\left(\mathrm{FD}^{+}\right) \mathrm{m} / z$ calcd for $\mathrm{C}_{78} \mathrm{H}_{95} \mathrm{~N}_{8} \mathrm{O}_{15}\left[\mathrm{M}+\mathrm{H}^{+}\right]$1383.6911, found 1383.6917 .

Precatenane 9:

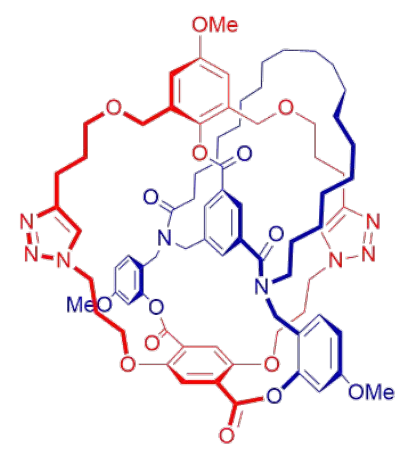

Alkene bismacrocycle 25 ( $99.2 \mathrm{mg}, 71.7 \mu \mathrm{mol}, 1.0$ equiv.) was dissolved in $8 \mathrm{~mL}$ of THF under a $\mathrm{N}_{2}$ atmosphere, to which $4 \mathrm{~mL}$ of absolute MeOH were added, followed by $10 \% \mathrm{Pd} / \mathrm{C}$ ( $22.9 \mathrm{mg}, 21.5 \mu \mathrm{mol}, 0.3$ equiv.). The suspension was sparged with $\mathrm{H}_{2}$ for 10 minutes, then stirred for 20 hours at $50^{\circ} \mathrm{C}$ under a $\mathrm{H}_{2}$ atmosphere. The crude reaction mixture was then cooled to room temperature, flushed with $\mathrm{N}_{2}$ and filtered over celite with some THF. The crude product was purified by column chromatography (AcOEt/ $\mathrm{CH}_{2} \mathrm{Cl}_{2}$ 1:1 $\rightarrow$ 7:3 $\rightarrow$ 8:2) to give precatenane 9 (65.4 $\mathrm{mg}$, $66 \%)$ as a colorless glass.

$R_{\mathrm{f}}=0.20\left(\mathrm{AcOEt} / \mathrm{CH}_{2} \mathrm{Cl}_{2} 7: 3\right) ;{ }^{1} \mathrm{H} \mathrm{NMR}\left(400 \mathrm{MHz}, \mathrm{CDCl}_{3}\right) \delta 8.07(\mathrm{~s}, 0.5 \mathrm{H}), 7.97(\mathrm{~s}, 1 \mathrm{H})$, $7.90-7.66(\mathrm{~m}, 3 \mathrm{H}), 7.29(\mathrm{~d}, \mathrm{~J}=8.5 \mathrm{~Hz}, 1 \mathrm{H}), 6.94(\mathrm{t}, \mathrm{J}=2.9 \mathrm{~Hz}, 1 \mathrm{H}), 6.91-6.74(\mathrm{~m}$, $5 \mathrm{H}), 6.69(\mathrm{~s}, 0.5 \mathrm{H}), 6.51(\mathrm{~s}, 0.5 \mathrm{H}), 6.19(\mathrm{~s}, 0.5 \mathrm{H}), 5.95(\mathrm{dd}, \mathrm{J}=14.9,8.6 \mathrm{~Hz}, 1 \mathrm{H}), 5.42$ $(\mathrm{s}, 0.5 \mathrm{H}), 5.32(\mathrm{~s}, 0.5 \mathrm{H}), 5.02(\mathrm{~d}, \mathrm{~J}=17.0 \mathrm{~Hz}, 0.5 \mathrm{H}), 4.89-4.02(\mathrm{~m}, 14.5 \mathrm{H}), 3.87(\mathrm{~s}$, $3 \mathrm{H}), 3.84-3.78(\mathrm{~m}, 6 \mathrm{H}), 3.74(\mathrm{dd}, \mathrm{J}=15.1,9.2 \mathrm{~Hz}, 1.5 \mathrm{H}), 3.57-3.36(\mathrm{~m}, 3 \mathrm{H}), 3.30(\mathrm{dd}, \mathrm{J}=15.6,9.4 \mathrm{~Hz}, 1 \mathrm{H}), 3.03-$ $2.91(\mathrm{~m}, 0.5 \mathrm{H}), 2.75-2.07(\mathrm{~m}, 12 \mathrm{H}), 2.06-1.86(\mathrm{~m}, 2 \mathrm{H}), 1.85-1.55(\mathrm{~m}, 5 \mathrm{H}), 1.54-0.60(\mathrm{~m}, 32 \mathrm{H}) ;{ }^{13} \mathrm{C} \mathrm{NMR}(101$ $\left.\mathrm{MHz}, \mathrm{CDCl}_{3}\right) \delta 174.1,169.2,168.8,163.8,163.7,163.6,163.3,162.3,162.1,160.8,159.6,159.6,157.3,157.2,152.3$, $152.1,151.6,151.6,150.5,150.5,149.4,149.4,147.1,146.9,146.1,145.3,142.8,142.2,140.6,140.0,138.3,138.1$, $136.0,136.0,132.8,132.7,132.3,132.2,131.4,131.2,131.0,130.6,128.6,128.2,127.7,127.4,125.3,125.1,124.4$, $124.2,124.0,123.9,121.4,121.1,121.0,121.0,121.0,120.8,120.0,119.6,119.4,118.7,116.1,116.1,116.0,115.6$, $115.3,115.2,111.9,111.5,111.5,109.8,109.6,108.1,108.0,71.4,71.2,71.0,70.7,70.6,70.5,70.3,69.7,66.5,66.4$, 66.0, 65.4, 55.7, 55.7, 55.6, 52.0, 51.7, 46.4, 46.2, 46.1, 46.0, 45.9, 45.6, 45.1, 44.9, 43.4, 43.1, 33.6, 33.4, 31.9, 30.3, $30.2,30.1,30.0,30.0,29.8,29.8,29.7,29.7,29.6,29.5,29.5,29.4,29.4,29.3,29.3,29.2,29.1,29.1,29.0,28.9,28.9$, $28.8,28.4,28.2,28.0,27.8,27.6,27.3,27.0,25.7,25.5,24.9,22.7,22.4,22.1,21.7,14.1$; IR (neat): $v_{\max } / \mathrm{cm}^{-1} 2926$, 2853, 1740, 1619, 1505, 1465, 1432, 1414, 1389, 1363, 1320, 1289, 1254, 1186, 1151, 1105, 1049, 965, 913, 730; HRMS $\left(\mathrm{ESI}^{+}\right) \mathrm{m} / z$ calcd for $\mathrm{C}_{78} \mathrm{H}_{97} \mathrm{~N}_{8} \mathrm{O}_{15}\left[\mathrm{M}+\mathrm{H}^{+}\right]$1385.7068, found 1385.7056 .

\section{Trisphenol [2]catenane 10:}

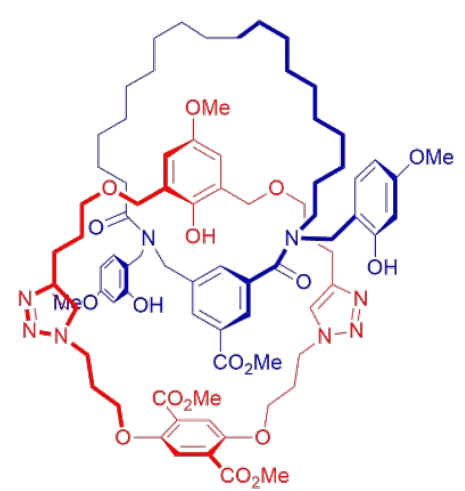

Precatenane 9 (65.4 mg, $47.2 \mu \mathrm{mol}, 1.0$ equiv.) was dissolved in $5 \mathrm{~mL}$ of warm anhydrous THF under a $\mathrm{N}_{2}$ atmosphere, to which $5 \mathrm{~mL}$ of anhydrous $\mathrm{MeOH}$ was added, followed by $\mathrm{K}_{2} \mathrm{CO}_{3}(2.45 \mathrm{~g}, 17.7 \mathrm{mmol}, 375$ equiv.). The resulting suspension was stirred vigorously for 5 hours at room temperature and then neutralized with ca. $2 \mathrm{~mL} \mathrm{AcOH}$ and subsequently dry loaded onto silica. The crude product was purified by column chromatography ( $\mathrm{MeOH}$ in $\mathrm{CH}_{2} \mathrm{Cl}_{2} 1 \% \rightarrow$ $2 \% \rightarrow 5 \%$ ) to give trisphenol [2]catenane 10 (58.8 mg, 84\%) as a colorless glass. $R_{\mathrm{f}}=0.42\left(\mathrm{MeOH}\right.$ in $\left.\mathrm{CH}_{2} \mathrm{Cl}_{2} 5 \%\right) ;{ }^{1} \mathrm{H} \mathrm{NMR}(400 \mathrm{MHz}, \mathrm{CDCl} 3) \delta 9.95-9.49(\mathrm{~m}, 2 \mathrm{H})$, $8.09-7.85(\mathrm{~m}, 1 \mathrm{H}), 7.72(\mathrm{~s}, 0.5 \mathrm{H}), 7.62-7.29(\mathrm{~m}, 3.5 \mathrm{H}), 7.19(\mathrm{~d}, \mathrm{~J}=14.0 \mathrm{~Hz}$, $1 \mathrm{H}), 7.10-6.97(\mathrm{~m}, 1 \mathrm{H}), 6.76(\mathrm{~d}, \mathrm{~J}=8.1 \mathrm{~Hz}, 1 \mathrm{H}), 6.67-6.24(\mathrm{~m}, 5 \mathrm{H}), 5.34(\mathrm{~s}$, $0.5 \mathrm{H}), 4.95-4.24(\mathrm{~m}, 9 \mathrm{H}), 4.15(\mathrm{~d}, \mathrm{~J}=9.9 \mathrm{~Hz}, 2.5 \mathrm{H}), 3.88(\mathrm{~d}, \mathrm{~J}=12.5 \mathrm{~Hz}, 9 \mathrm{H})$, $3.78(\mathrm{~d}, \mathrm{~J}=13.6 \mathrm{~Hz}, 6 \mathrm{H}), 3.66(\mathrm{~s}, 3 \mathrm{H}), 3.55(\mathrm{~s}, 4 \mathrm{H}), 3.21(\mathrm{~s}, 3 \mathrm{H}), 2.67(\mathrm{~s}, 2 \mathrm{H}), 2.53$ $-2.12(\mathrm{~m}, 6.5 \mathrm{H}), 1.92(\mathrm{~s}, 4 \mathrm{H}), 1.62(\mathrm{~d}, \mathrm{~J}=7.9 \mathrm{~Hz}, 4 \mathrm{H}), 1.44(\mathrm{~s}, 1.5 \mathrm{H}), 1.36-0.69$ (m, 37H); ${ }^{13} \mathrm{C}$ NMR $\left(101 \mathrm{MHz}, \mathrm{CDCl}_{3}\right) \delta$ 177.3, 176.2, 172.3, 165.6, 165.1, 165.0, 161.6, 161.5, 157.6, 157.6, 152.3, 151.6, 146.7, 146.1, 137.9, 136.4, 132.5, 132.3, 131.2, 127.2, 127.0, 125.1, 123.4, 121.9, 115.9, 115.3, 114.4, 106.0, 105.4, 102.6, 76.2, 71.7, 70.9, 70.3, 68.8, 63.9, 63.5, 55.6, 55.3, 52.5, 52.4, 52.3, 49.3, 49.1, 45.7, 45.6, 45.4, 33.8, $32.9,31.9,31.9,31.9,29.8,29.7,29.7,29.6,29.5,29.5,29.5,29.4,29.3,29.3,29.2,29.1,28.9,28.7,28.5,27.2,27.1$, 25.1, 24.8, 22.7, 22.7, 22.6, 14.1; IR (neat): $v_{\max } / \mathrm{cm}^{-1} 3138,2925,2853,1723,1615,1506,1465,1436,1389,1290$, $1239,1207,1161,1101,1038,964,848,787,774,732$; HRMS $\left(\right.$ FD $\left.^{+}\right) \mathrm{m} / z$ calcd for $\mathrm{C}_{81} \mathrm{H}_{109} \mathrm{~N}_{8} \mathrm{O}_{18}\left[\mathrm{M}+\mathrm{H}^{+}\right]$1481.7854, found 1481.7841 .

[2]catenane 2:

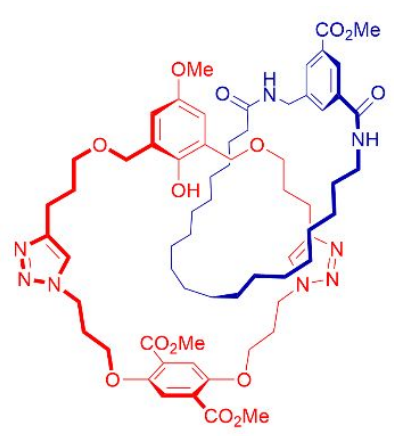

Trisphenol [2]catenane 10 (58.8 mg, $39.7 \mu \mathrm{mol}, 1.0$ equiv.) was dissolved in $2 \mathrm{~mL}$ of $\mathrm{CHCl}_{3}$ and the solution was cooled to $0^{\circ} \mathrm{C}$. Subsequently, $\mathrm{Et}_{3} \mathrm{SiH}(63.4 \mu \mathrm{L}, 397 \mu \mathrm{mol}$, 10 equiv.) was added, followed by dropwise addition of $2 \mathrm{~mL}$ of TFA. The mixture was allowed to warm to room temperature and stirred over a period of 2 hours before $24 \mathrm{~mL}$ of toluene were added and the crude was quickly concentrated in vacuo to remove all solvents. The crude product was purified by column 
chromatography ( $\mathrm{MeOH}$ in $\mathrm{CH}_{2} \mathrm{Cl}_{2} 1 \% \rightarrow 2 \% \rightarrow 3 \% \rightarrow 4 \%$ ) to give [2]catenane 2 (32.6 $\mathrm{mg}, 68 \%$ ) as a colorless glass. $R_{\mathrm{f}}=0.32\left(\mathrm{MeOH}\right.$ in $\left.\mathrm{CH}_{2} \mathrm{Cl}_{2} 5 \%\right) ;{ }^{1} \mathrm{H}$ NMR $\left(500 \mathrm{MHz}, \mathrm{CDCl}_{3}\right) \delta 8.27(\mathrm{~s}, 1 \mathrm{H}), 7.99(\mathrm{~s}, 1 \mathrm{H}), 7.81(\mathrm{~s}, 1 \mathrm{H}), 7.55(\mathrm{~s}, 1 \mathrm{H}), 7.40$ $(\mathrm{s}, 2 \mathrm{H}), 7.34(\mathrm{~s}, 1 \mathrm{H}), 6.73(\mathrm{~s}, 1 \mathrm{H}), 6.60(\mathrm{~s}, 1 \mathrm{H}), 4.70(\mathrm{~s}, 4 \mathrm{H}), 4.50(\mathrm{~s}, 2 \mathrm{H}), 4.38(\mathrm{~s}, 3 \mathrm{H}), 4.18(\mathrm{~s}, 1 \mathrm{H}), 3.87(\mathrm{~d}, \mathrm{~J}=9.5 \mathrm{~Hz}$, $12 \mathrm{H}), 3.73(\mathrm{~s}, 4 \mathrm{H}), 3.59(\mathrm{~s}, 2 \mathrm{H}), 3.41(\mathrm{~s}, 2 \mathrm{H}), 3.24(\mathrm{~s}, 1 \mathrm{H}), 3.02(\mathrm{~s}, 1 \mathrm{H}), 2.74(\mathrm{~s}, 2 \mathrm{H}), 2.56-2.28(\mathrm{~m}, 6 \mathrm{H}), 1.96(\mathrm{~s}, 4 \mathrm{H})$, $1.62(\mathrm{~s}, 2 \mathrm{H}), 1.42(\mathrm{~s}, 2 \mathrm{H}), 1.25(\mathrm{~s}, 4 \mathrm{H}), 1.19-0.83(\mathrm{~m}, 28 \mathrm{H}) ;{ }^{13} \mathrm{C} \mathrm{NMR}\left(75 \mathrm{MHz}, \mathrm{CDCl}_{3}\right) \delta 173.4,166.4,166.3,165.1$, 165.0, 152.5, 151.6, 151.4, 147.9, 146.8, 146.6, 139.1, 135.6, 132.4, 131.8, 130.5, 127.6, 124.6, 124.1, 124.0, 123.4, $122.5,116.1,114.8,113.6,71.1,70.5,70.2,69.3,64.1,63.8,55.8,52.3,52.3,45.9,45.8,43.3,40.0,36.1,29.6,29.5$, $29.4,29.3,29.3,29.3,29.1,29.1,29.0,29.0,28.5,26.7,25.4,22.4,22.2$; IR (neat): $v_{\max } / \mathrm{cm}^{-1} 3331,2925,2852,1722$, 1655, 1600, 1541, 1505, 1436, 1408, 1388, 1311, 1239, 1206, 1132, 1099, 1057, 980, 918, 836, 788, 754, 730, 645, 508; HRMS $\left(\mathrm{FD}^{+}\right) \mathrm{m} / \mathrm{z}$ calcd for $\mathrm{C}_{65} \mathrm{H}_{93} \mathrm{~N}_{8} \mathrm{O}_{14}\left[\mathrm{M}+\mathrm{H}^{+}\right] 1209.6805$, found 1209.6889 .

Prerotaxane 12:

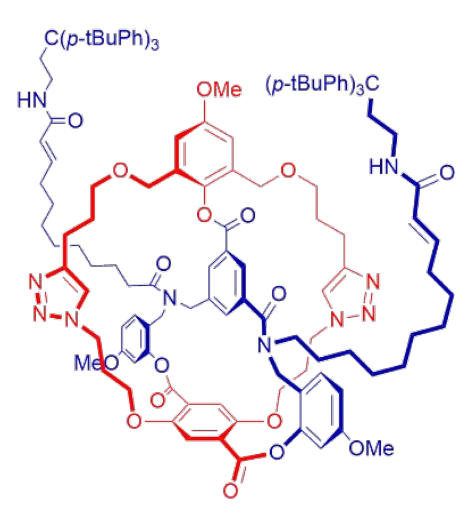

Triazole macrocycle 1 (100 mg, $70.8 \mu \mathrm{mol}, 1.0$ equiv.) and alkene stopper $\mathbf{1 1}^{7}$ (144 mg, $283 \mu \mathrm{mol}, 4$ equiv.) were dissolved in $23 \mathrm{~mL}$ of $\mathrm{CH}_{2} \mathrm{Cl}_{2}$ under a $\mathrm{N}_{2}$ atmosphere and the resulting solution was sparged with $\mathrm{N}_{2}$ for 30 minutes. Then Grubbs $2^{\text {nd }}$ generation catalyst $(12.0 \mathrm{mg}, 14.2 \mu \mathrm{mol}, 0.2$ equiv.) was added and sparging was continued for 10 minutes. After stirring for 19 hours at reflux the crude reaction mixture was concentrated, dry loaded onto silica and purified by column chromatography (EtOAc in $\mathrm{CH}_{2} \mathrm{Cl}_{2} 20 \% \rightarrow 40 \% \rightarrow 60 \%$ $\rightarrow 80 \%)$ to give prerotaxane $12(127.2 \mathrm{mg}, 76 \%)$ as a brown glass.

$R_{\mathrm{f}}=0.38(\mathrm{EtOAC} / \mathrm{PE} 9: 1) ;{ }^{1} \mathrm{H} \mathrm{NMR}\left(400 \mathrm{MHz}, \mathrm{CDCl}_{3}\right) \delta 8.17-7.74(\mathrm{~m}, 4 \mathrm{H}), 7.72-$ $7.55(\mathrm{~m}, 1 \mathrm{H}), 7.38-7.13(\mathrm{~m}, 24 \mathrm{H}), 6.98(\mathrm{~s}, 1 \mathrm{H}), 6.93-6.57(\mathrm{~m}, 8 \mathrm{H}), 6.54-6.46$ $(\mathrm{m}, 0.5 \mathrm{H}), 6.30-6.17(\mathrm{~m}, 0.5 \mathrm{H}), 6.10-5.93(\mathrm{~m}, 1 \mathrm{H}), 5.62-5.28(\mathrm{~m}, 3 \mathrm{H}), 5.28$ $-5.02(\mathrm{~m}, 2 \mathrm{H}), 4.73-4.05(\mathrm{~m}, 14 \mathrm{H}), 3.90-3.68(\mathrm{~m}, 11 \mathrm{H}), 3.64-3.21(\mathrm{~m}, 4 \mathrm{H})$, $3.13(\mathrm{~s}, 4 \mathrm{H}), 2.87-2.65(\mathrm{~m}, 4 \mathrm{H}), 2.62-1.89(\mathrm{~m}, 18 \mathrm{H}), 1.77-1.58(\mathrm{~m}, 4 \mathrm{H}), 1.53$ - 0.99 (m, 80H); ${ }^{13} \mathrm{C}$ NMR (101 MHz, $\left.\mathrm{CDCl}_{3}\right) \delta 173.5,171.1,168.8,166.0,166.0,165.9,165.1,163.3,162.5,160.8$, $159.5,157.1,152.7,151.5,151.2,150.5,150.2,149.2,148.5,148.4,147.2,147.0,146.6,144.4,144.3,144.2,144.0$, 144.0, 142.6, 142.3, 142.1, 139.9, 138.1, 137.8, 136.0, 132.8, 132.0, 131.6, 131.1, 130.5, 129.6, 129.0, 128.7, 128.5, $125.9,125.5,124.7,124.4,123.6,123.5,123.5,121.4,121.0,120.7,120.0,119.5,118.6,115.6,115.2,115.0,112.0$, 111.4, 109.8, 108.0, 76.2, 71.0, 70.7, 70.5, 66.5, 65.6, 60.4, 55.7, 55.7, 55.7, 55.6, 54.5, 54.5, 51.6, 46.0, 45.3, 43.3, $41.5,40.0,37.2,34.5,34.3,34.1,33.8,33.2,31.9,31.9,31.6,31.4,31.2,29.9,29.7,29.4,29.2,29.2,29.1,29.0,28.9$, $28.6,28.2,27.8,26.7,25.4,25.3,25.1,22.7,22.5,22.2,21.9,21.1,14.2,14.2$; IR (neat): $v_{\max } / \mathrm{cm}^{-1} 2957,2929,2858$, $1741,1670,1620,1506,1463,1440,1413,1392,1362,1320,1287,1255,1185,1152,1106,1049,1016,910,840$, 821, 730, 646, 588; HRMS (FD $) \mathrm{m} / z$ calcd for $\mathrm{C}_{148} \mathrm{H}_{185} \mathrm{~N}_{10} \mathrm{O}_{17}\left[\mathrm{M}+\mathrm{H}^{+}\right]$2374.3914, found 2374.3952.

\section{Trisphenol [2]rotaxane 13:}

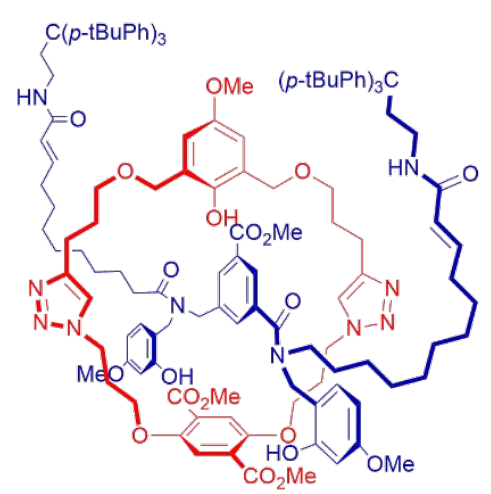

Prerotaxane 12 (127 mg, $53.6 \mu \mathrm{mol}, 1.0$ equiv.) was dissolved in $6 \mathrm{~mL}$ of anhydrous THF under a $\mathrm{N}_{2}$ atmosphere, to which $6 \mathrm{~mL}$ of anhydrous $\mathrm{MeOH}$ were added, followed by $\mathrm{K}_{2} \mathrm{CO}_{3}(2.78 \mathrm{~g}, 20.1 \mathrm{mmol}, 375$ equiv.). After stirring at room temperature for 6 hours, the crude reaction mixture was neutralized with ca. $2 \mathrm{~mL}$ of $\mathrm{AcOH}$ and dry loaded onto silica. The crude product was purified by column chromatography (EtOAc in $\mathrm{CH}_{2} \mathrm{Cl}_{2} 30 \% \rightarrow 40 \% \rightarrow 60 \%$ ) to give trisphenol [2]rotaxane $13(99.2 \mathrm{mg}, 75 \%)$ as a colorless glass.

$R_{\mathrm{f}}=0.42\left(\mathrm{EtOAC} / \mathrm{CH}_{2} \mathrm{Cl}_{2} 1: 1\right) ;{ }^{1} \mathrm{H} \mathrm{NMR}\left(400 \mathrm{MHz}, \mathrm{CDCl}_{3}\right) \delta 9.83-9.58(\mathrm{~m}, 2 \mathrm{H})$, $8.02(\mathrm{~s}, 1 \mathrm{H}), 7.94-7.83(\mathrm{~m}, 1 \mathrm{H}), 7.80(\mathrm{~s}, 0.5 \mathrm{H}), 7.70(\mathrm{~s}, 0.5 \mathrm{H}), 7.65-7.58(\mathrm{~m}$, $1 \mathrm{H}), 7.54(\mathrm{~s}, 1 \mathrm{H}), 7.46(\mathrm{~s}, 1 \mathrm{H}), 7.35-6.98(\mathrm{~m}, 27 \mathrm{H}), 6.86-6.68(\mathrm{~m}, 2 \mathrm{H}), 6.61$ $(\mathrm{d}, \mathrm{J}=9.9 \mathrm{~Hz}, 2 \mathrm{H}), 6.54(\mathrm{~d}, \mathrm{~J}=12.9 \mathrm{~Hz}, 1 \mathrm{H}), 6.51-6.38(\mathrm{~m}, 2 \mathrm{H}), 6.31(\mathrm{~d}, \mathrm{~J}=8.4$ $\mathrm{Hz}, 2 \mathrm{H}), 5.54-5.31(\mathrm{~m}, 2 \mathrm{H}), 5.14-5.02(\mathrm{~m}, 1 \mathrm{H}), 4.79-4.26(\mathrm{~m}, 14 \mathrm{H}), 4.02-$ $3.86(\mathrm{~m}, 5 \mathrm{H}), 3.86-3.65(\mathrm{~m}, 14 \mathrm{H}), 3.65-3.57(\mathrm{~m}, 3 \mathrm{H}), 3.57-3.45(\mathrm{~m}, 4 \mathrm{H})$, $3.23-3.10(\mathrm{~m}, 4 \mathrm{H}), 2.95-2.84(\mathrm{~m}, 1 \mathrm{H}), 2.78(\mathrm{t}, \mathrm{J}=7.3 \mathrm{~Hz}, 3 \mathrm{H}), 2.72-2.50(\mathrm{~m}, 6 \mathrm{H}), 2.49-2.28(\mathrm{~m}, 5 \mathrm{H}), 2.19(\mathrm{t}, \mathrm{J}=$ $7.6 \mathrm{~Hz}, 1 \mathrm{H}), 2.08(\mathrm{q}, \mathrm{J}=7.3 \mathrm{~Hz}, 2 \mathrm{H}), 1.94-1.77(\mathrm{~m}, 4 \mathrm{H}), 1.75-1.53(\mathrm{~m}, 4 \mathrm{H}), 1.27(\mathrm{~d}, \mathrm{~J}=4.9 \mathrm{~Hz}, 79 \mathrm{H}) ;{ }^{13} \mathrm{C} \mathrm{NMR}(101$ $\left.\mathrm{MHz}_{1} \mathrm{CDCl}_{3}\right) \delta 176.1,176.1,172.0,171.8,171.2,166.0,165.9,165.7,165.6,165.5,165.2,165.1,161.6,161.6,161.6$, $161.5,157.6,157.5,152.5,152.4,151.4,148.5,148.5,148.2,147.9,147.0,146.9,144.3,144.3,144.1,144.0,144.0$, $142.5,142.1,137.5,137.4,136.7,136.5,132.4,132.4,132.0,131.3,131.3,129.6,128.9,128.7,127.4,127.3,124.7$, $124.6,124.5,124.3,124.1,123.8,123.7,123.6,123.5,122.7,122.5,122.4,116.2,114.3,114.2,114.1,114.1,114.0$, 114.0, 105.9, 105.6, 102.7, 102.6, 102.5, 98.4, 70.3, 70.2, 70.2, 70.1, 64.2, 62.0, 60.4, 55.6, 55.6, 55.3, 55.3, 55.2, $54.5,54.2,54.1,52.6,52.2,52.2,51.7,49.9,49.8,48.7,46.0,45.8,45.3,44.7,40.0,39.9,37.2,34.3,34.3,34.1,33.2$, $33.1,31.9,31.7,31.5,31.4,31.2,30.8,29.7,29.5,29.4,29.3,29.3,29.2,29.1,29.0,28.3,28.2,28.2,28.0,27.7,26.9$, 
26.7, 25.1, 25.0, 23.4, 22.7, 22.4, 21.8, 21.1, 14.2, 1.0; IR (neat): $v_{\max } / \mathrm{cm}^{-1} 2952,2930,2859,1725,1671,1619,1507$, $1462,1435,1408,1390,1362,1289,1268,1239,1204,1162,1102,1058,1037,1016,965,910,841,822,787,731$, 646 , 588; HRMS (FD $\left.{ }^{+}\right) \mathrm{m} / \mathrm{z}$ calcd for $\mathrm{C}_{151} \mathrm{H}_{197} \mathrm{~N}_{10} \mathrm{O}_{20}\left[\mathrm{M}+\mathrm{H}^{+}\right] 2470.4700$, found 2470.4694 .

[2]rotaxane 3:

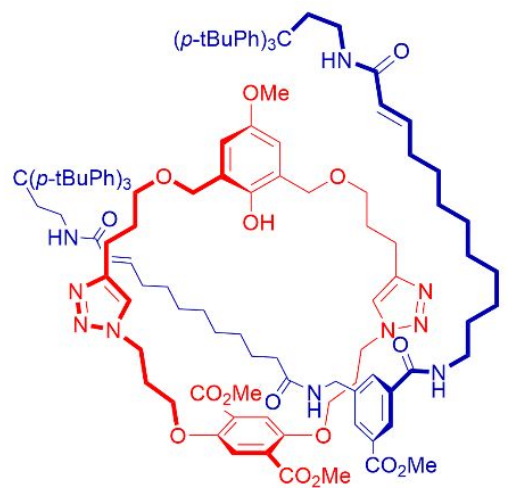

Trisphenol [2]rotaxane 13 (83.9 mg, $34.0 \mu \mathrm{mol}, 1.0$ equiv.) was dissolved in $2.5 \mathrm{~mL}$ of $\mathrm{CHCl}_{3}$ and the solution was cooled to $0^{\circ} \mathrm{C}$. Subsequently, $\mathrm{Et}_{3} \mathrm{SiH}$ ( $54.2 \mu \mathrm{L}, 340 \mu \mathrm{mol}, 10$ equiv.) was added, followed by dropwise addition of $2.5 \mathrm{~mL}$ of TFA. The mixture was allowed to warm to room temperature and stirred over a period of 2 hours before $60 \mathrm{~mL}$ of toluene were added and the crude was quickly concentrated in vacuo to remove all solvents. The crude product was purified by column chromatography ( $\mathrm{MeOH}$ in $\mathrm{CH}_{2} \mathrm{Cl}_{2} 1 \%$ $\rightarrow 2 \% \rightarrow 3 \%$ ) to give [2] rotaxane 3 (37.5 mg, 50\%) as a colorless glass.

$R_{\mathrm{f}}=0.32\left(\mathrm{MeOH}\right.$ in $\left.\mathrm{CH}_{2} \mathrm{Cl}_{2} 5 \%\right) ;{ }^{1} \mathrm{H}$ NMR $\left(500 \mathrm{MHz}, \mathrm{CDCl}_{3}\right) \delta 8.27(\mathrm{~s}, 0.5 \mathrm{H})$, $8.13(\mathrm{~s}, 0.5 \mathrm{H}), 7.95(\mathrm{~s}, 0.5 \mathrm{H}), 7.85(\mathrm{~s}, 0.5 \mathrm{H}), 7.83(\mathrm{~s}, 0.5 \mathrm{H}), 7.72(\mathrm{~s}, 0.5 \mathrm{H}), 7.66$ $(\mathrm{s}, 1 \mathrm{H}), 7.60(\mathrm{~s}, 1 \mathrm{H}), 7.34(\mathrm{~s}, 1 \mathrm{H}), 7.29(\mathrm{~s}, 1 \mathrm{H}), 7.28-7.12(\mathrm{~m}, 25 \mathrm{H}), 6.91(\mathrm{~s}$, $0.5 \mathrm{H}), 6.72(\mathrm{~s}, 1 \mathrm{H}), 6.66-6.42(\mathrm{~m}, 3.5 \mathrm{H}), 5.76(\mathrm{~s}, 0.5 \mathrm{H}), 5.66(\mathrm{~s}, 0.5 \mathrm{H}), 5.51$ $-5.38(\mathrm{~m}, 2 \mathrm{H}), 5.06-4.92(\mathrm{~m}, 1 \mathrm{H}), 4.77-4.54(\mathrm{~m}, 4 \mathrm{H}), 4.48-4.26(\mathrm{~m}, 5 \mathrm{H})$, $4.09(\mathrm{~s}, 1 \mathrm{H}), 3.99-3.88(\mathrm{~m}, 4 \mathrm{H}), 3.86(\mathrm{~s}, 1.5 \mathrm{H}), 3.83(\mathrm{~s}, 1.5 \mathrm{H}), 3.80(\mathrm{~s}, 3 \mathrm{H}), 3.76(\mathrm{~s}, 3 \mathrm{H}), 3.68-3.61(\mathrm{~m}, 3 \mathrm{H}), 3.55-$ $3.41(\mathrm{~m}, 4 \mathrm{H}), 3.35(\mathrm{~s}, 1 \mathrm{H}), 3.16(\mathrm{~s}, 2 \mathrm{H}), 3.05(\mathrm{~s}, 2 \mathrm{H}), 2.97(\mathrm{~s}, 1 \mathrm{H}), 2.82-2.56(\mathrm{~m}, 8 \mathrm{H}), 2.37(\mathrm{~s}, 4 \mathrm{H}), 2.19(\mathrm{~s}, 1 \mathrm{H}), 2.12-$ $2.03(\mathrm{~m}, 2 \mathrm{H}), 1.96-1.73(\mathrm{~m}, 7 \mathrm{H}), 1.67-1.48(\mathrm{~m}, 2 \mathrm{H}), 1.43-0.76(\mathrm{~m}, 78 \mathrm{H}) ;{ }^{13} \mathrm{C} \mathrm{NMR}\left(75 \mathrm{MHz}, \mathrm{CDCl}_{3}\right) \delta 173.3,166.2$, 166.0, 165.2, 165.1, 152.5, 152.4, 151.5, 148.5, 148.4, 147.8, 146.9, 144.5, 144.0, 143.5, 139.7, 139.1, 135.4, 131.4, $131.3,130.8,130.4,128.7,127.2,124.7,124.7,124.6,123.8,123.6,123.4,122.7,116.3,114.3,113.9,70.2,64.3$, $55.7,54.6,54.4,52.3,52.2,45.9,42.9,42.6,40.3,40.0,37.1,36.5,35.9,34.3,31.9,31.8,31.4,29.7,29.5,29.3,29.1$, 29.0, 28.2, 28.1, 27.0, 25.7, 25.4, 22.1; IR (neat): $v_{\max } / \mathrm{cm}^{-1} 3296,2956,2930,2861,1725,1668,1541,1508,1461$, $1437,1407,1390,1362,1268,1241,1207,1141,1105,1058,1016,978,910,841,822,788,732,647,588$; HRMS $\left(\right.$ FD $\left.^{+}\right) \mathrm{m} / \mathrm{z}$ calcd for $\mathrm{C}_{135} \mathrm{H}_{181} \mathrm{~N}_{10} \mathrm{O}_{16}\left[\mathrm{M}+\mathrm{H}^{+}\right]$2198.3652, found 2198.3578.

Trivial triazole macrocycle 26:

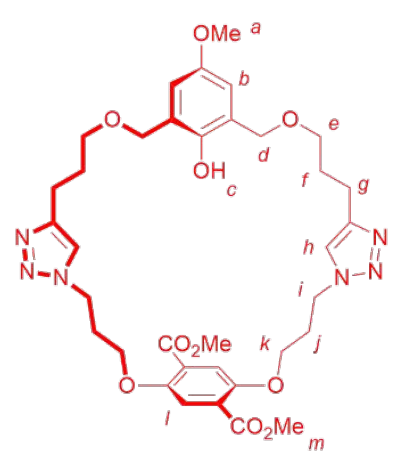

Methyl ester template 24 ( $294 \mathrm{mg}, 0.750 \mathrm{mmol}, 1.0$ equiv.), dialkyne ring fragment 5 (237 mg, $0.750 \mathrm{mmol}, 1.0$ equiv.) and DiPEA ( $52.2 \mu \mathrm{L}, 0.300 \mathrm{mmol}, 0.4$ equiv.) were dissolved in $750 \mathrm{~mL}$ of $\mathrm{CH}_{2} \mathrm{Cl}_{2}$ under a $\mathrm{N}_{2}$ atmosphere. After sparging the solution with $\mathrm{N}_{2}$ for 2 hours, $\mathrm{Cu}(\mathrm{MeCN})_{4} \mathrm{BF}_{4}(70.8 \mathrm{mg}, 0.225 \mathrm{mmol}, 0.3$ equiv.) was added and the resulting reaction mixture was heated at reflux for 16 hours. The crude mixture was then concentrated in vacuo, dry loaded onto silica and the product was purified by column chromatography ( $\mathrm{MeOH}$ in $\mathrm{CH}_{2} \mathrm{Cl}_{2} 2 \% \rightarrow 3 \% \rightarrow 4 \%$ ) to give trivial triazole macrocycle $26(34.7 \mathrm{mg}, 6.5 \%)$ as a colorless glass. Note that when using TBTA in place of DiPEA higher yields could be obtained, but the product could not be completely separated from TBTA.

$R_{\mathrm{f}}=0.23\left(\mathrm{MeOH}\right.$ in $\left.\mathrm{CH}_{2} \mathrm{Cl}_{2} 5 \%\right) ;{ }^{1} \mathrm{H}$ NMR $\left(500 \mathrm{MHz}, \mathrm{CDCl}_{3}\right) \delta 7.61(\mathrm{~s}, 1 \mathrm{H}), 7.35(\mathrm{~s}, 2 \mathrm{H})$, $7.31(\mathrm{~s}, 2 \mathrm{H}), 6.65(\mathrm{~s}, 2 \mathrm{H}), 4.66(\mathrm{t}, \mathrm{J}=5.9 \mathrm{~Hz}, 4 \mathrm{H}), 4.50(\mathrm{~s}, 4 \mathrm{H}), 3.90(\mathrm{~s}, 6 \mathrm{H}), 3.83(\mathrm{t}, \mathrm{J}=$ $5.6 \mathrm{~Hz}, 4 \mathrm{H}), 3.74(\mathrm{~s}, 3 \mathrm{H}), 3.51(\mathrm{t}, \mathrm{J}=6.1 \mathrm{~Hz}, 4 \mathrm{H}), 2.76(\mathrm{t}, \mathrm{J}=7.7 \mathrm{~Hz}, 4 \mathrm{H}), 2.39(\mathrm{t}, \mathrm{J}=5.9 \mathrm{~Hz}, 4 \mathrm{H}), 1.94(\mathrm{p}, \mathrm{J}=6.4 \mathrm{~Hz}, 4 \mathrm{H})$; ${ }^{13} \mathrm{C} \mathrm{NMR}\left(101 \mathrm{MHz}, \mathrm{CDCl}_{3}\right) \delta 165.3,152.6,151.6,148.2,147.0,124.6,124.1,122.8,116.3,114.1,70.4,69.8,64.4$, 55.9, 52.4, 45.9, 29.4, 29.3, 22.4; IR (neat): $v_{\max } / \mathrm{cm}^{-1} 3382,3136,2949,2866,1724,1653,1616,1551,1466,1436$, $1408,1387,1356,1309,1237,1207,1150,1101,1053,976,916,868,788,729,646,529,482 ; \mathrm{HRMS}_{\left(\mathrm{FD}^{+}\right)} \mathrm{m} / \mathrm{z}$ calcd for $\mathrm{C}_{35} \mathrm{H}_{44} \mathrm{~N}_{6} \mathrm{O}_{10}\left[\mathrm{M}^{\bullet+}\right]$ 708.3113, found 708.3096.

Amide 27:

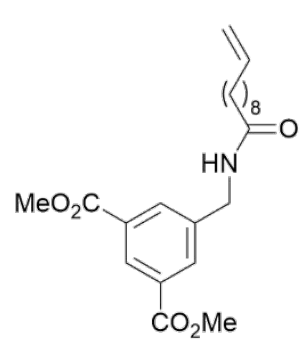

Azido ester 14 (600 mg, $2.40 \mathrm{mmol}, 1.0$ equiv.) was dissolved in $20 \mathrm{~mL}$ of $\mathrm{Et}_{2} \mathrm{O}$, to which $\mathrm{PPh}_{3}$ (694 mg, $2.64 \mathrm{mmol}, 1.1$ equiv.) was added, followed by $50 \mu \mathrm{L}$ of $\mathrm{H}_{2} \mathrm{O}$. After stirring at room temperature for 24 hours, the crude amine was concentrated in vacuo, diluted in $30 \mathrm{~mL}$ of anhydrous $\mathrm{CH}_{2} \mathrm{Cl}_{2}$ under a $\mathrm{N}_{2}$ atmosphere and cooled to $0^{\circ} \mathrm{C}$. To the crude amine solution, $\mathrm{Et}_{3} \mathrm{~N}(1.33 \mathrm{~mL}, 9.60 \mathrm{mmol}, 4.0$ equiv.) was added, followed by dropwise addition of a solution of freshly made 10 -undecenoyl chloride in $10 \mathrm{~mL}$ of anhydrous $\mathrm{CH}_{2} \mathrm{Cl}_{2}$. 10 Undecenoyl chloride was obtained by dissolving 10-undecenoic acid ( $884 \mathrm{mg}, 4.8 \mathrm{mmol}$, 2.0 equiv.) in $5 \mathrm{~mL}$ of $\mathrm{CHCl}_{3}$ and stirring at $60^{\circ} \mathrm{C}$ for 2 hours after addition of $\mathrm{SOCl}_{2}(0.700$ $\mathrm{mL}, 9.60 \mathrm{mmol}, 4.0$ equiv.), then concentrating the mixture in vacuo and diluting it under a $\mathrm{N}_{2}$ atmosphere. Once addition of 10-undecenoyl chloride was complete, the mixture was allowed to warm to 
room temperature and stirred for 24 hours. The crude product was dry loaded onto silica and purified by column chromatography (PE/EtOAc 2:1 $\rightarrow 1: 1 \rightarrow 1: 2$ ) to give amide $\mathbf{2 7}(710 \mathrm{mg}, 76 \%$ ) as a white powder.

$R_{\mathrm{f}}=0.39(\mathrm{PE} / \mathrm{EtOAC} 1: 1) ;{ }^{1} \mathrm{H}$ NMR $\left(400 \mathrm{MHz}, \mathrm{CDCl}_{3}\right) \delta 8.56(\mathrm{t}, \mathrm{J}=1.6 \mathrm{~Hz}, 1 \mathrm{H}), 8.12(\mathrm{~d}, \mathrm{~J}=1.6 \mathrm{~Hz}, 2 \mathrm{H}), 5.92(\mathrm{~s}, 1 \mathrm{H}), 5.87$ $-5.72(\mathrm{~m}, 1 \mathrm{H}), 5.02-4.88(\mathrm{~m}, 2 \mathrm{H}), 4.53(\mathrm{~d}, \mathrm{~J}=6.0 \mathrm{~Hz}, 2 \mathrm{H}), 3.93(\mathrm{~s}, 6 \mathrm{H}), 2.28-2.19(\mathrm{~m}, 2 \mathrm{H}), 2.07-1.97(\mathrm{~m}, 2 \mathrm{H})$, $1.72-1.60(\mathrm{~m}, 2 \mathrm{H}), 1.42-1.21(\mathrm{~m}, 10 \mathrm{H}) ;{ }^{13} \mathrm{C}$ NMR $\left(101 \mathrm{MHz}, \mathrm{CDCl}_{3}\right) \delta 173.2,166.0,139.6,139.2,132.8,131.0$, $129.8,114.2,52.4,42.8,36.7,33.8,29.3,29.3,29.1,28.9,25.7$; IR (neat): $v_{\max } / \mathrm{cm}^{-1} 3283,2918,2849,1720,1634$, $1539,1434,1325,1271,1245,1205,1127,1108,992,915,753,721 ; H_{R M S}\left(F D^{+}\right) \mathrm{m} / z$ calcd for $\mathrm{C}_{22} \mathrm{H}_{31} \mathrm{~N}_{1} \mathrm{O}_{5}\left(\mathrm{M}^{\bullet+}\right)$ 389.2197 , found 389.2216 .

\section{Carboxylic acid 28:}

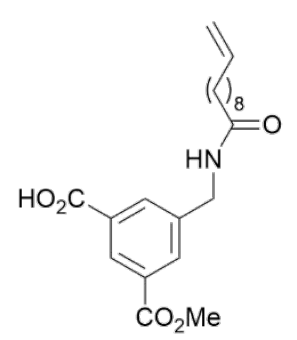

Dimethyl ester 27 (710 mg, $1.82 \mathrm{mmol}, 1.0$ equiv.) was dissolved in $28 \mathrm{~mL}$ of absolute $\mathrm{MeOH}$, to which $1 \mathrm{M} \mathrm{NaOH}(1.86 \mathrm{~mL}, 1.91 \mathrm{mmol}, 1.05$ equiv.) was added dropwise. After stirring under reflux for 19 hours the crude reaction mixture was cooled to room temperature and acidified to $\mathrm{pH} 1$ with $1 \mathrm{M} \mathrm{KHSO}_{4}$ and then partitioned between $80 \mathrm{~mL} \mathrm{H}_{2} \mathrm{O}$ and $80 \mathrm{~mL}$ EtOAc. The aqueous layer was then extracted $2 \times 40 \mathrm{~mL}$ EtOAc and the combined organic layers were dried over $\mathrm{MgSO}_{4}$ and concentrated in vacuo. The crude product was dry loaded onto silica and purified by column chromatography (EtOAc/PE 1:1 $\rightarrow$ 3:1 $\rightarrow$ EtOAc ) to give carboxylic acid 28 (551 $\mathrm{mg}, 81 \%)$ as a white solid.

$R_{\mathrm{f}}=0.20$ (streaks) (EtOAc); ${ }^{1} \mathrm{H}$ NMR $\left(400 \mathrm{MHz}, \mathrm{CD}_{3} \mathrm{OD}\right) \delta 8.47(\mathrm{~s}, 1 \mathrm{H}), 8.16(\mathrm{~s}, 1 \mathrm{H}), 8.12(\mathrm{~s}$, $1 \mathrm{H}), 5.87-5.70(\mathrm{~m}, 1 \mathrm{H}), 5.00-4.86(\mathrm{~m}, 2 \mathrm{H}), 4.47(\mathrm{~s}, 2 \mathrm{H}), 3.93(\mathrm{~s}, 3 \mathrm{H}), 2.27(\mathrm{t}, \mathrm{J}=7.4 \mathrm{~Hz}, 2 \mathrm{H}), 2.01(\mathrm{q}, \mathrm{J}=6.8 \mathrm{~Hz}, 2 \mathrm{H})$, $1.63(\mathrm{q}, \mathrm{J}=7.2 \mathrm{~Hz}, 2 \mathrm{H}), 1.41-1.21(\mathrm{~m}, 10 \mathrm{H}) ;{ }^{13} \mathrm{C} \mathrm{NMR}\left(101 \mathrm{MHz}, \mathrm{CD}_{3} \mathrm{OD}\right)$ 8 175.0, 167.1, 166.1, 140.3, 138.7, 132.7, 132.2, 131.4, 130.6, 129.0, 113.4, 51.6, 42.0, 35.7, 33.5, 29.1, 29.0, 28.9, 28.8, 28.7, 25.7; IR (neat): $v_{\max } / \mathrm{cm}^{-1} 3293$, $3076,2925,2853,2478,1724,1639,1545,1459,1434,1351,1302,1238,1206,1125,1030,994,909,756,722$, $684,632,570,478$; HRMS $\left(\mathrm{FD}^{+}\right) \mathrm{m} / z$ calcd for $\mathrm{C}_{21} \mathrm{H}_{30} \mathrm{~N}_{1} \mathrm{O}_{5}\left[\mathrm{M}+\mathrm{H}^{+}\right]$376.2118, found 376.2109.

Bisamide 29:

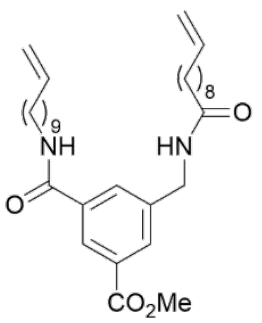

Carboxylic acid 28 ( $311 \mathrm{mg}, 0.830 \mathrm{mmol}, 1.0$ equiv.) was suspended in $30 \mathrm{~mL}$ of anhydrous $\mathrm{CH}_{2} \mathrm{Cl}_{2}$ under a $\mathrm{N}_{2}$ atmosphere, to which DiPEA ( $289 \mu \mathrm{L}, 1.66 \mathrm{mmol}, 2$ equiv.), HBTU (472 $\mathrm{mg}, 1.24 \mathrm{mmol}, 1.5$ equiv.) and 10-Undecen-1-amine ${ }^{3}$ (169 mg, $0.996 \mathrm{mmol}, 1.2$ equiv.) were added. After stirring at room temperature for 20 hours, the crude mixture was washed with $2 \times 20 \mathrm{~mL} 1 \mathrm{M} \mathrm{HCl}, 2 \times 20 \mathrm{~mL} \mathrm{H}_{2} \mathrm{O}$, dried over $\mathrm{MgSO}_{4}$ and concentrated in vacuo. The crude product was dry loaded onto silica and purified by column chromatography (EtOAc in $\mathrm{CH}_{2} \mathrm{Cl}_{2}$ $10 \% \rightarrow 20 \% \rightarrow 40 \%$ ) to give bisamide 29 (329 mg, 75\%) as a white solid.

$R_{\mathrm{f}}=0.16\left(\right.$ EtOAc in $\left.\mathrm{CH}_{2} \mathrm{Cl}_{2} 20 \%\right) ;{ }^{1} \mathrm{H} \mathrm{NMR}\left(400 \mathrm{MHz}, \mathrm{CDCl}_{3}\right) \delta 8.06(\mathrm{~s}, 1 \mathrm{H}), 7.83(\mathrm{~s}, 1 \mathrm{H}), 7.70(\mathrm{~s}$, $1 \mathrm{H}), 6.90(\mathrm{t}, \mathrm{J}=5.8 \mathrm{~Hz}, 1 \mathrm{H}), 6.86(\mathrm{t}, \mathrm{J}=5.9 \mathrm{~Hz}, 1 \mathrm{H}), 5.84-5.68(\mathrm{~m}, 2 \mathrm{H}), 5.00-4.85(\mathrm{~m}, 4 \mathrm{H})$, $4.29(\mathrm{~d}, \mathrm{~J}=6.0 \mathrm{~Hz}, 2 \mathrm{H}), 3.82(\mathrm{~s}, 3 \mathrm{H}), 3.36(\mathrm{q}, \mathrm{J}=6.8 \mathrm{~Hz}, 2 \mathrm{H}), 2.21(\mathrm{t}, \mathrm{J}=7.7 \mathrm{~Hz}, 2 \mathrm{H}), 2.06-1.93(\mathrm{~m}, 4 \mathrm{H}), 1.66-1.53$ (m, 4H), $1.40-1.18(\mathrm{~m}, 22 \mathrm{H}) ;{ }^{13} \mathrm{C}$ NMR $\left(101 \mathrm{MHz}, \mathrm{CDCl}_{3}\right) \delta 173.7,166.6,166.1,139.7,139.1,139.1,135.4,130.9$, 130.5, 130.4, 126.6, 114.2, 114.1, 52.3, 42.6, 40.3, 36.5, 33.8, 33.8, 29.5, 29.5, 29.4, 29.4, 29.3, 29.1, 28.9, 28.9, 27.1, 25.7; IR (neat): $v_{\max } / \mathrm{cm}^{-1} 3264,3080,2921,2851,1727,1635,1600,1553,1465,1451,1434,1422,1374,1343$, $1289,1254,1202,1106,991,909,755,720,665$; HRMS $\left(F^{+}\right) \mathrm{m} / z$ calcd for $\mathrm{C}_{32} \mathrm{H}_{50} \mathrm{~N}_{2} \mathrm{O}_{4}\left[\mathrm{M}^{\bullet+}\right] 526.3765$, found 526.3757 .

Trivial alkene macrocycle 30:

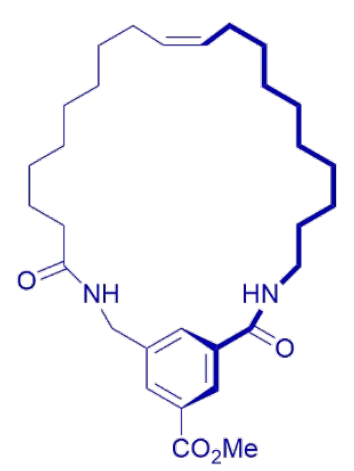

Bisalkene 29 ( $329 \mathrm{mg}, 0.625 \mathrm{mmol}, 1.0$ equiv.) was dissolved in $\mathrm{CH}_{2} \mathrm{Cl}_{2}$ under a $\mathrm{N}_{2}$ atmosphere and the solution was sparged with $\mathrm{N}_{2}$ for 1 hour. Then Grubbs $2^{\text {nd }}$ generation catalyst $(53.1 \mathrm{mg}, 62.5 \mu \mathrm{mol}, 0.1$ equiv.) was added and the mixture was heated at reflux for 16 hours. The crude product was then concentrated in vacuo, dry loaded onto silica and purified by column chromatography $\left(\mathrm{CH}_{2} \mathrm{Cl}_{2} / \mathrm{EtOAc}\right.$ 9:1 $\rightarrow$ 8:2 $\rightarrow$ $6: 4)$ to give trivial alkene macrocycle $30(62.7 \mathrm{mg}, 20 \%)$ as a white solid.

$R_{\mathrm{f}}=0.19\left(\mathrm{CH}_{2} \mathrm{Cl}_{2} / \mathrm{EtOAc} 6: 4\right) ;{ }^{1} \mathrm{H}$ NMR $\left(400 \mathrm{MHz}, \mathrm{CDCl}_{3}+\mathrm{CD}_{3} \mathrm{OD}\right) \delta 8.29(\mathrm{~s}, 1 \mathrm{H}), 7.95$ (s, $1 \mathrm{H}), 7.79-7.71(\mathrm{~m}, 1 \mathrm{H}), 7.44(\mathrm{~s}, 1 \mathrm{H}), 7.23(\mathrm{~s}, 1 \mathrm{H}), 5.34-5.19(\mathrm{~m}, 2 \mathrm{H}), 4.39(\mathrm{~s}, 2 \mathrm{H}), 3.88$ $(\mathrm{s}, 3 \mathrm{H}), 3.36(\mathrm{t}, \mathrm{J}=6.9 \mathrm{~Hz}, 2 \mathrm{H}), 2.19(\mathrm{t}, \mathrm{J}=7.6 \mathrm{~Hz}, 2 \mathrm{H}), 2.03-1.86(\mathrm{~m}, 4 \mathrm{H}), 1.62-1.52$ $(\mathrm{m}, 4 \mathrm{H}), 1.38-1.12(\mathrm{~m}, 22 \mathrm{H}) ;{ }^{13} \mathrm{C} \mathrm{NMR}\left(101 \mathrm{MHz}, \mathrm{CDCl}_{3}+\mathrm{CD}_{3} \mathrm{OD}\right) \delta$ 174.6, 167.2, 166.6, 139.7, 135.7, 131.4, 130.6, 130.6, 130.4, 130.1, 130.0, 127.5, 52.4, 42.6, 40.2, 36.3, 32.3, $32.1,29.5,29.2,29.2,29.1,29.1,29.0,29.0,28.9,28.7,28.6,28.1,27.0,26.9,26.8,25.5 ;$ IR (neat): $v_{\max } / \mathrm{cm}^{-1} 3262,3085,2919,2849,1727,1656,1634,1605,1570,1550,1452,1434,1422,1373,1342$, $1290,1254,1201,1106,1035,967,755,717,665,508$; $\mathrm{HRMS}\left(\mathrm{FD}^{+}\right) \mathrm{m} / \mathrm{z}$ calcd for $\mathrm{C}_{30} \mathrm{H}_{46} \mathrm{~N}_{2} \mathrm{O}_{4}\left[\mathrm{M}^{\bullet+}\right.$ ] 498.3452, found 498.3432 . 


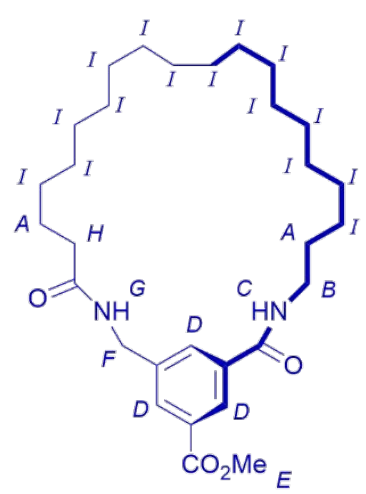

Trivial alkene macrocycle 30 ( $59.9 \mathrm{mg}, 120 \mu \mathrm{mol}, 1.0$ equiv.) was dissolved in a warm 2:1 THF/MeOH mixture. Once cold, 10\%w/w Pd/C ( $25.6 \mathrm{mg}, 24 \mu \mathrm{mol}, 0.2$ equiv.) was added under a $\mathrm{N}_{2}$ atmosphere and the suspension was sparged with $\mathrm{H}_{2}$ for 10 minutes, then stirred at $50^{\circ} \mathrm{C}$ under a $\mathrm{H}_{2}$ atmosphere for 20 hours. The crude mixture was then cooled to room temperature, flushed with $\mathrm{N}_{2}$ and filtered over celite. To ensure complete dissolution of the product, before filtration the mixture was diluted with an equal amount of $\mathrm{THF} / \mathrm{MeOH}$ and warmed. The crude product was concentrated in vacuo and purified by column chromatography (EtOAc in $\mathrm{CH}_{2} \mathrm{Cl}_{2} 10 \%$ $\rightarrow 20 \% \rightarrow 40 \%)$ to give trivial bisamide macrocycle 31 (56.6 mg, 94\%) as a white solid. $R_{\mathrm{f}}=0.19\left(\mathrm{CH}_{2} \mathrm{Cl}_{2} /\right.$ EtOAc 6:4); ${ }^{1} \mathrm{H}$ NMR $\left(500 \mathrm{MHz}, \mathrm{CDCl}_{3}\right) \delta 8.28(\mathrm{~s}, 1 \mathrm{H}), 8.11-8.03(\mathrm{~m}$, $1 \mathrm{H}), 7.95(\mathrm{~s}, 1 \mathrm{H}), 6.30(\mathrm{~s}, 1 \mathrm{H}), 5.98(\mathrm{~s}, 1 \mathrm{H}), 4.50(\mathrm{~d}, \mathrm{~J}=6.0 \mathrm{~Hz}, 2 \mathrm{H}), 3.93(\mathrm{~s}, 3 \mathrm{H}), 3.47(\mathrm{q}$, $\mathrm{J}=6.8 \mathrm{~Hz}, 1.5 \mathrm{H}), 3.22(\mathrm{q}, \mathrm{J}=7.2 \mathrm{~Hz}, 0.5 \mathrm{H}), 2.33(\mathrm{t}, \mathrm{J}=7.6 \mathrm{~Hz}, 0.5 \mathrm{H}), 2.24(\mathrm{t}, \mathrm{J}=7.6 \mathrm{~Hz}$, $1.5 \mathrm{H}), 1.64(\mathrm{q}, \mathrm{J}=7.4 \mathrm{~Hz}, 4 \mathrm{H}), 1.25(\mathrm{~d}, \mathrm{~J}=5.6 \mathrm{~Hz}, 26 \mathrm{H}), 0.87(\mathrm{q}, \mathrm{J}=6.7 \mathrm{~Hz}, 4 \mathrm{H}) ;{ }^{13} \mathrm{C} \mathrm{NMR}$ $\left(101 \mathrm{MHz} \mathrm{CDCl}_{3}\right) \delta 174.1,174.1,167.0,166.9,166.5,139.7,135.9,135.8,131.7,130.8,130.8,127.3,52.5,43.1$, $43.0,40.4,40.3,36.5,36.4,32.0,29.8,29.8,29.7,29.7,29.6,29.6,29.5,29.4,29.4,29.3,29.2,29.1,29.0,29.0,29.0$, 28.9, 28.9, 28.9, 28.8, 28.7, 28.7, 28.6, 28.6, 26.8, 25.4, 22.8, 22.7, 14.2, 1.2, 1.1; IR (neat): $v_{\max } / \mathrm{cm}^{-1} 3261,3087$, 2921, 2850, 1728, 1656, 1634, 1605, 1572, 1553, 1462, 1453, 1434, 1422, 1374, 1342, 1291, 1254, 1201, 1106, $1035,983,755,714,665$; HRMS $\left(F^{+}\right) \mathrm{m} / z$ calcd for $\mathrm{C}_{30} \mathrm{H}_{48} \mathrm{~N}_{2} \mathrm{O}_{4} \mathrm{Na}\left[\mathrm{M}+\mathrm{Na}^{+}\right]$523.3506, found 523.3525.

\section{Trivial thread 32:}

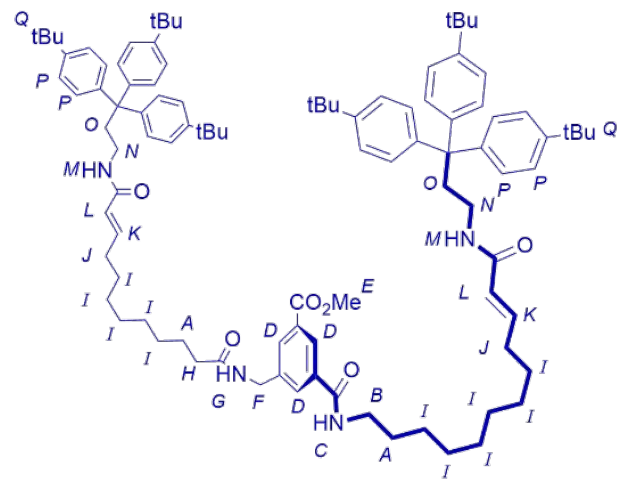

Bisalkene 29 (91.5 mg, $174 \mu \mathrm{mol}, 1.0$ equiv.) was dissolved in 43.5 $\mathrm{mL}$ of $\mathrm{CH}_{2} \mathrm{Cl}_{2}$ under a $\mathrm{N}_{2}$ atmosphere. After addition of alkene stopper $\mathbf{1 1}^{7}$ ( $355 \mathrm{mg}, 696 \mu \mathrm{mol}, 4.0$ equiv.) the solution was sparged with $\mathrm{N}_{2}$ for 30 minutes, then Grubbs $2^{\text {nd }}$ generation catalyst ( $29.5 \mathrm{mg}, 34.0 \mu \mathrm{mol}, 0.2$ equiv.) was added and the reaction mixture was stirred for 20 hours at reflux. Then the mixture was concentrated in vacuo and the product was purified by column chromatography (EtOAc in $\mathrm{CH}_{2} \mathrm{Cl}_{2} 10 \% \rightarrow 20 \% \rightarrow 30 \%$ ) to give trivial thread $32(179 \mathrm{mg}, 69 \%)$ as a brown glass.

$R_{\mathrm{f}}=0.35\left(\mathrm{MeOH}\right.$ in $\left.\mathrm{CH}_{2} \mathrm{Cl}_{2} 5 \%\right) ;{ }^{1} \mathrm{H}$ NMR $\left(500 \mathrm{MHz}, \mathrm{CDCl}_{3}\right) \delta 8.23(\mathrm{~s}$, $1 \mathrm{H}), 7.98(\mathrm{~s}, 1 \mathrm{H}), 7.87(\mathrm{~s}, 1 \mathrm{H}), 7.26-7.18(\mathrm{~m}, 24 \mathrm{H}), 6.86(\mathrm{t}, \mathrm{J}=5.7$ $\mathrm{Hz}, 1 \mathrm{H}), 6.76-6.64(\mathrm{~m}, 3 \mathrm{H}), 5.49(\mathrm{~d}, \mathrm{~J}=15.3 \mathrm{~Hz}, 2 \mathrm{H}), 5.30(\mathrm{t}, \mathrm{J}=5.9$ $\mathrm{Hz}, 1 \mathrm{H}), 5.24(\mathrm{t}, \mathrm{J}=5.9 \mathrm{~Hz}, 1 \mathrm{H}), 4.67(\mathrm{~d}, \mathrm{~J}=8.3 \mathrm{~Hz}, 0.5 \mathrm{H}), 4.40(\mathrm{~d}, \mathrm{~J}$ $=5.9 \mathrm{~Hz}, 1.5 \mathrm{H}), 3.85(\mathrm{~s}, 3 \mathrm{H}), 3.39(\mathrm{q}, \mathrm{J}=6.6 \mathrm{~Hz}, 2 \mathrm{H}), 3.11(\mathrm{q}, \mathrm{J}=6.5 \mathrm{~Hz}, 4 \mathrm{H}), 2.75(\mathrm{t}, \mathrm{J}=7.3 \mathrm{~Hz}, 4 \mathrm{H}), 2.19(\mathrm{t}, \mathrm{J}=7.6 \mathrm{~Hz}$, $2 \mathrm{H}), 2.07(\mathrm{q}, \mathrm{J}=7.1 \mathrm{~Hz}, 4 \mathrm{H}), 1.86(\mathrm{~d}, \mathrm{~J}=12.6 \mathrm{~Hz}, 1 \mathrm{H}), 1.68-1.51(\mathrm{~m}, 3 \mathrm{H}), 1.40-1.17(\mathrm{~m}, 76 \mathrm{H}) ;{ }^{13} \mathrm{C} \mathrm{NMR}(126 \mathrm{MHz}$, $\left.\mathrm{CDCl}_{3}\right) \delta 173.6,166.5,166.2,166.1,166.1,148.5,144.3,144.3,144.0,139.9,135.6,131.2,130.8,130.7,128.7$, $126.7,124.8,123.7,123.6,54.5,52.4,48.9,42.8,40.3,40.0,37.2,36.5,34.3,34.0,32.0,31.9,31.4,29.6,29.5,29.4$, $29.4,29.3,29.2,29.2,29.2,29.1,29.1,28.9,28.3,28.2,27.0,25.7,25.7,25.0$; IR (neat): $v_{\text {max }} / \mathrm{cm}^{-1} 3284,3082,2959$, 2928, 2856, 1728, 1641, 1546, 1509, 1459, 1394, 1362, 1269, 1205, 1112, 1016, 978, 910, 840, 821, 754, 733, 705, 587,577 ; HRMS $\left(\right.$ FD $\left.^{+}\right) \mathrm{m} / z$ calcd for $\mathrm{C}_{100} \mathrm{H}_{137} \mathrm{~N}_{4} \mathrm{O}_{6}\left[\mathrm{M}+\mathrm{H}^{+}\right]$1490.0533, found 1490.0489. 


\section{NMR Spectra}

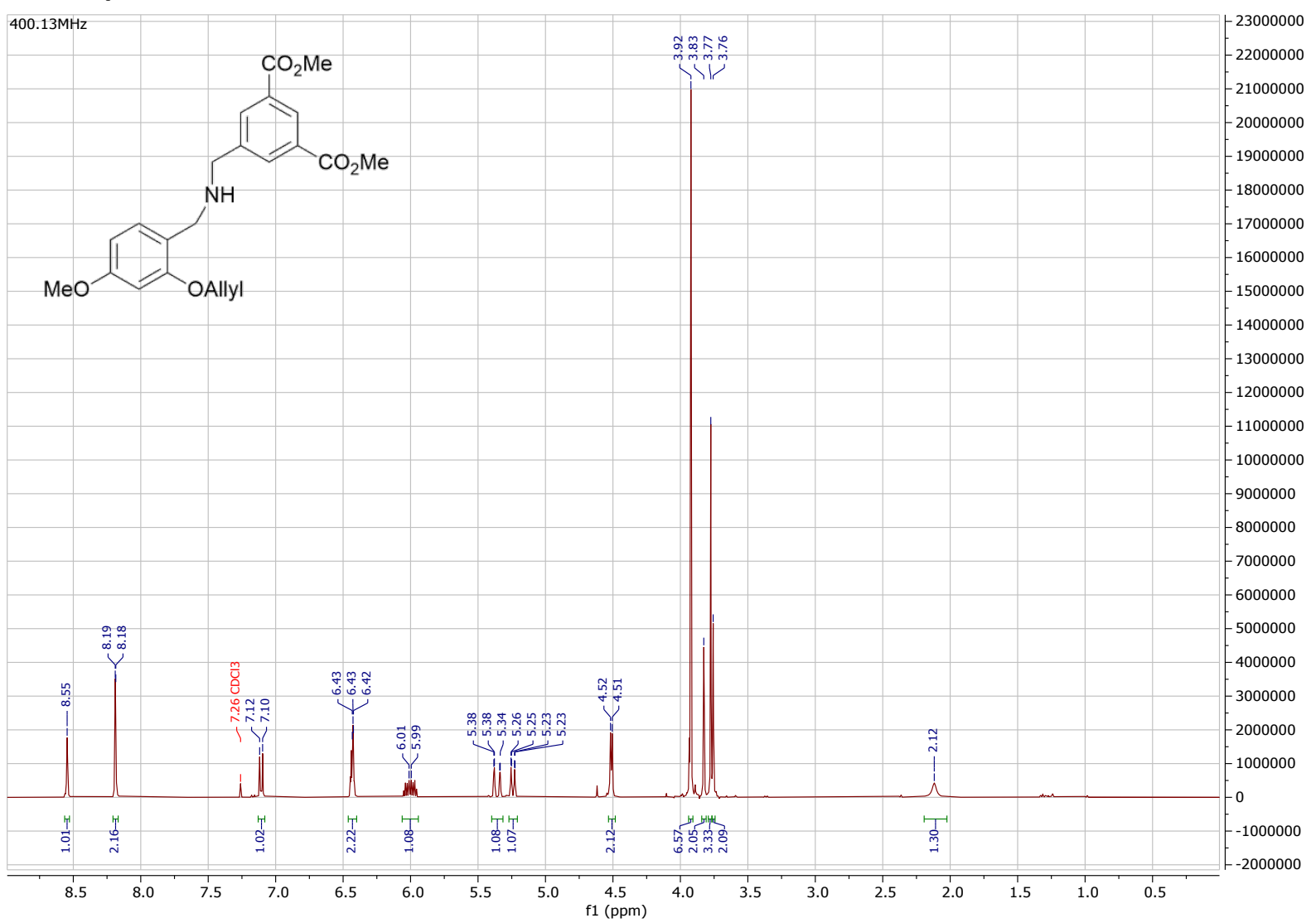

Figure S1. ${ }^{1} \mathrm{H}$ NMR spectrum of compound 16 in $\mathrm{CDCl}_{3}$ at $400 \mathrm{MHz}$.

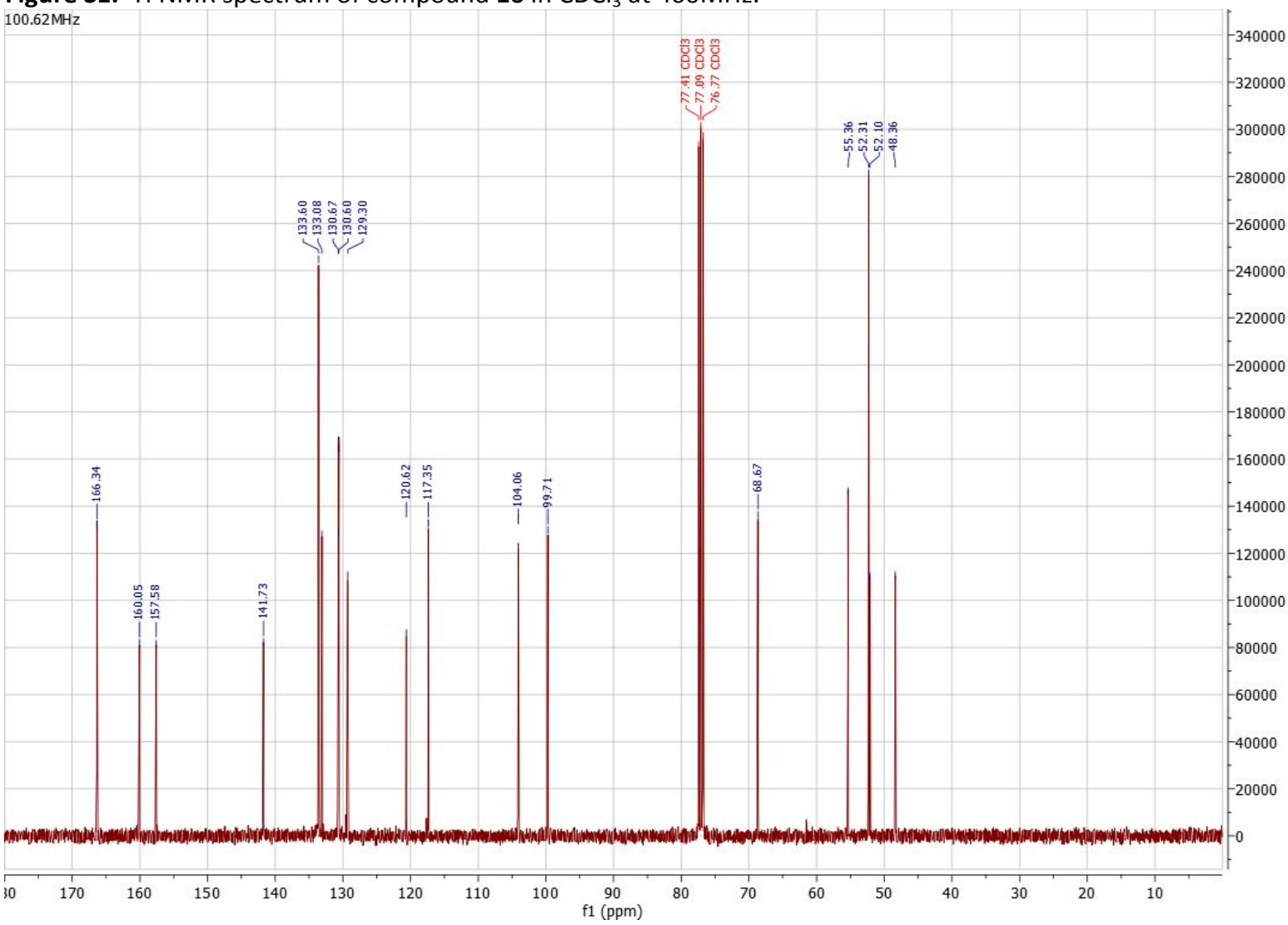

Figure S2. ${ }^{13} \mathrm{C}$ NMR spectrum of compound 16 in $\mathrm{CDCl}_{3}$ at $400 \mathrm{MHz}$. 


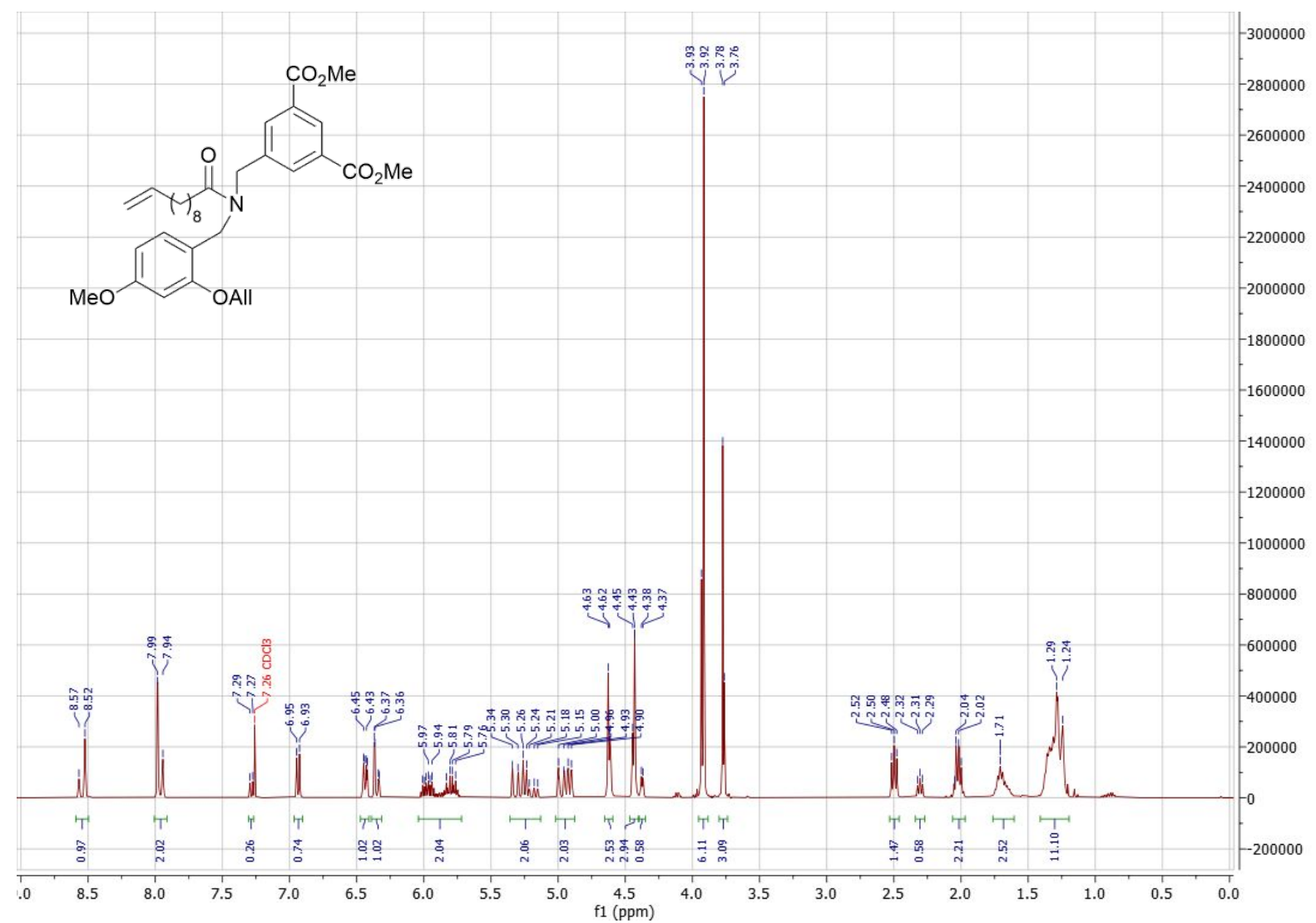

Figure S3. ${ }^{1} \mathrm{H}$ NMR spectrum of compound 17 in $\mathrm{CDCl}_{3}$ at $400 \mathrm{MHz}$.

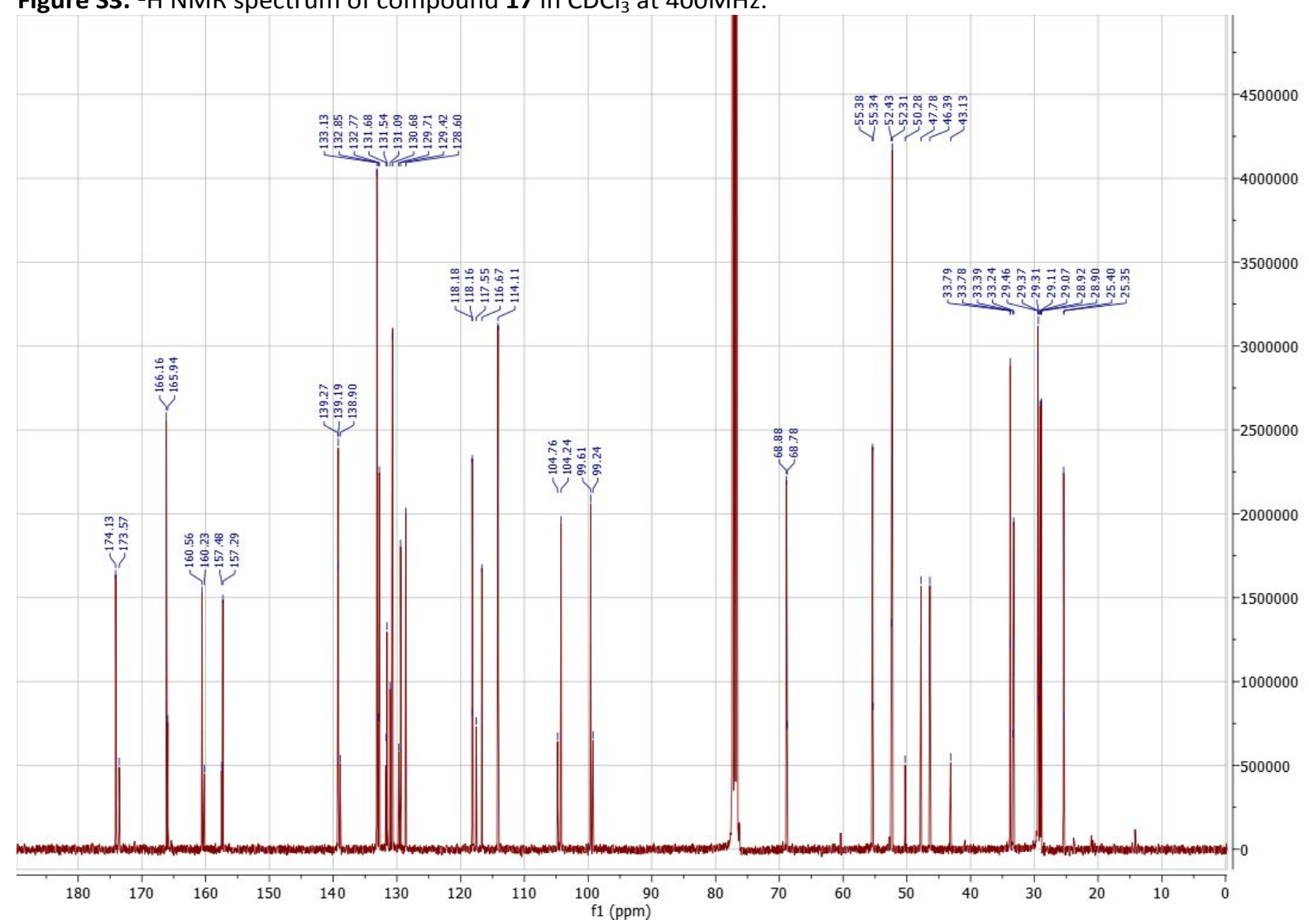

Figure S4. ${ }^{13} \mathrm{C}$ NMR spectrum of compound 17 in $\mathrm{CDCl}_{3}$ at $400 \mathrm{MHz}$. 


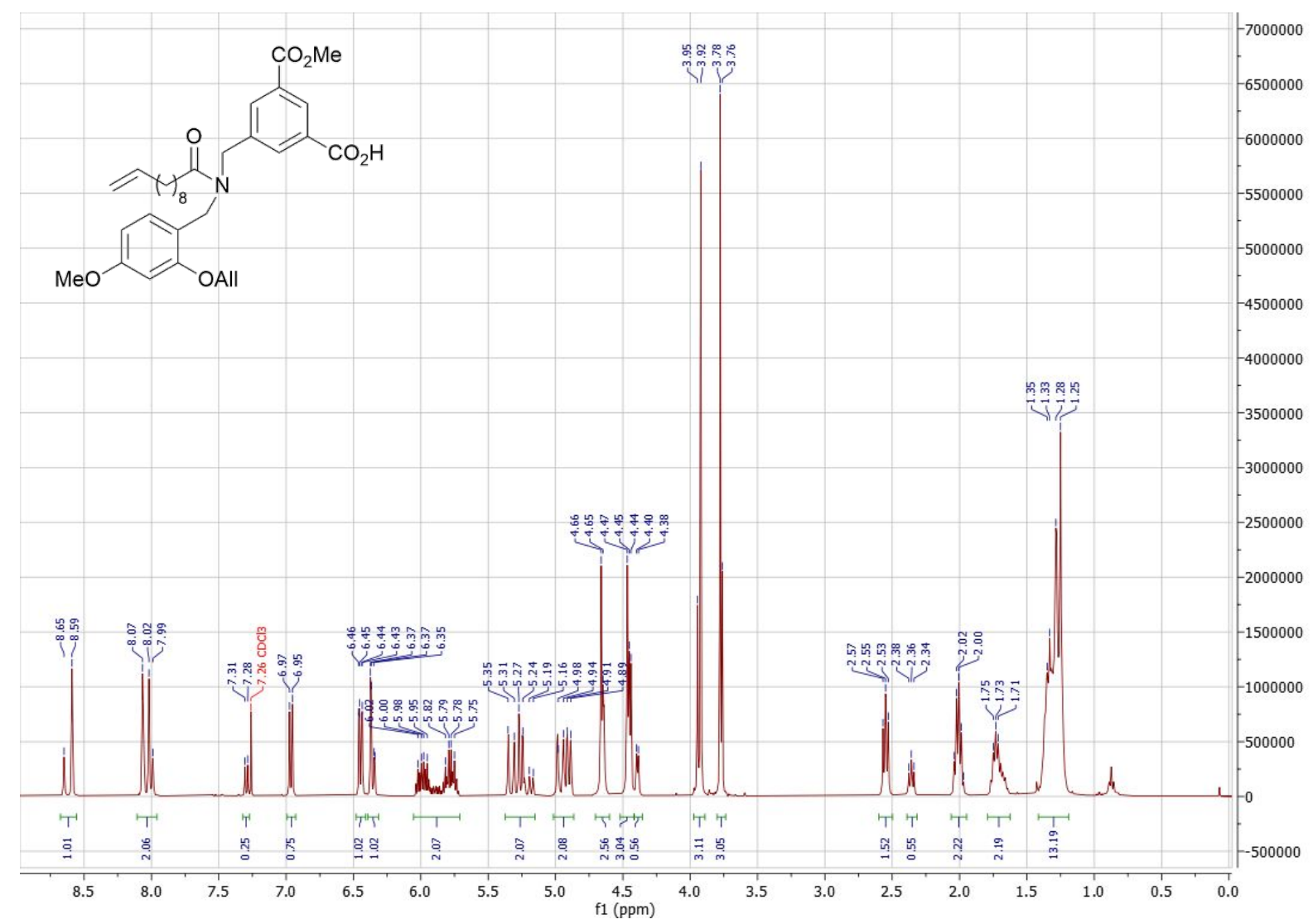

Figure S5. ${ }^{1} \mathrm{H}$ NMR spectrum of compound 18 in $\mathrm{CDCl}_{3}$ at $400 \mathrm{MHz}$.

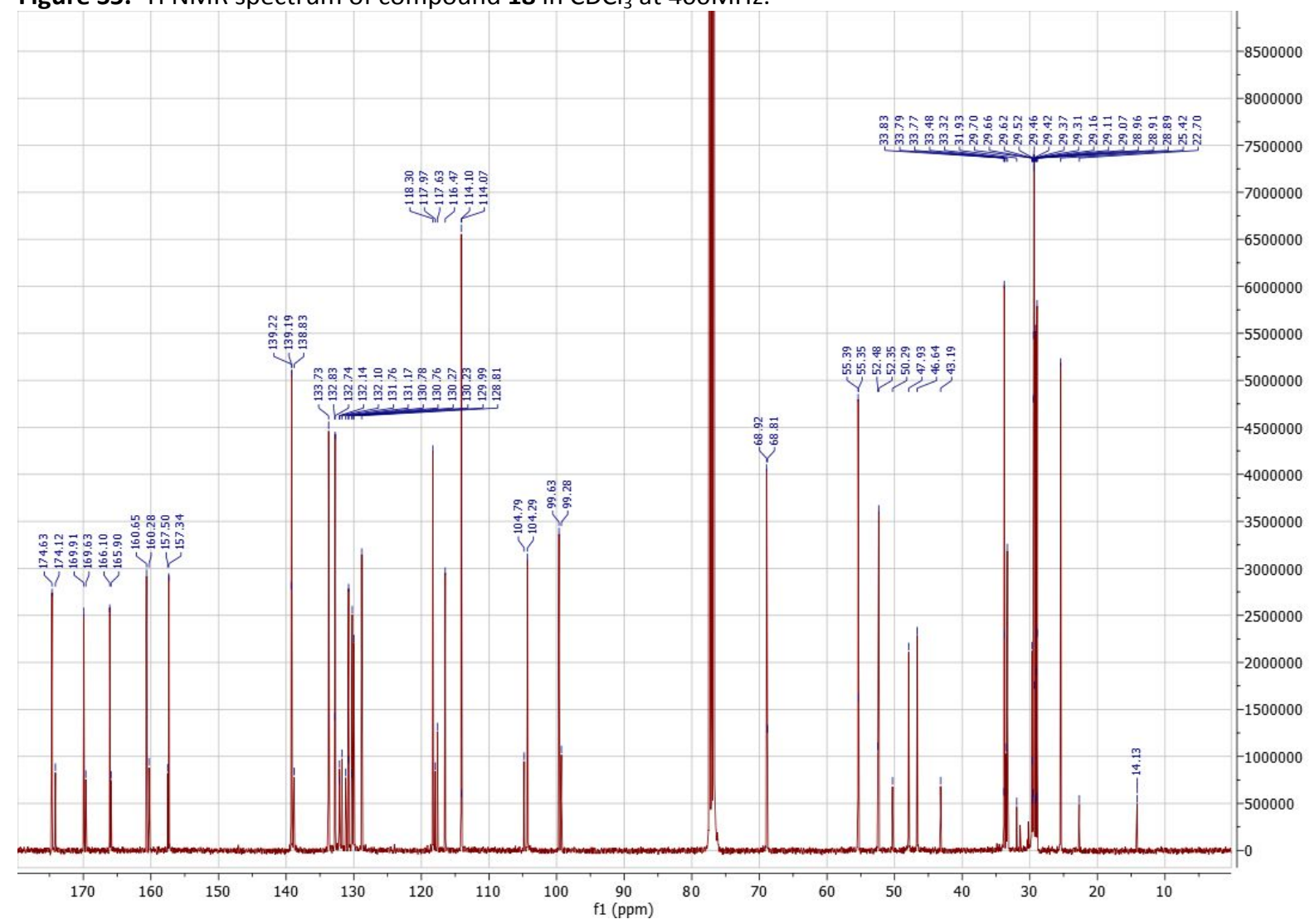

Figure S6. ${ }^{13} \mathrm{C}$ NMR spectrum of compound 18 in $\mathrm{CDCl}_{3}$ at $400 \mathrm{MHz}$. 


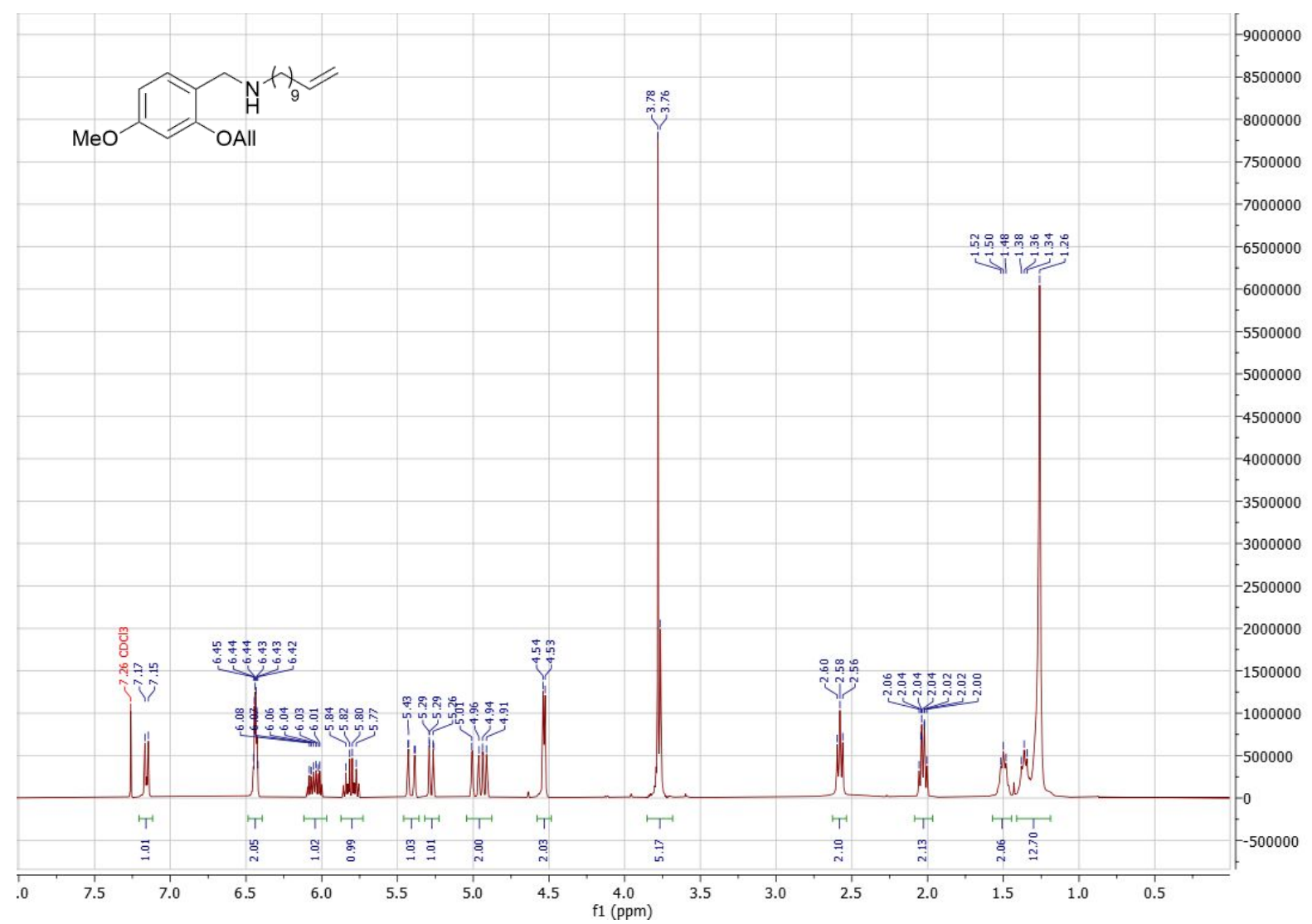

Figure S7. ${ }^{1} \mathrm{H}$ NMR spectrum of compound 19 in $\mathrm{CDCl}_{3}$ at $400 \mathrm{MHz}$.

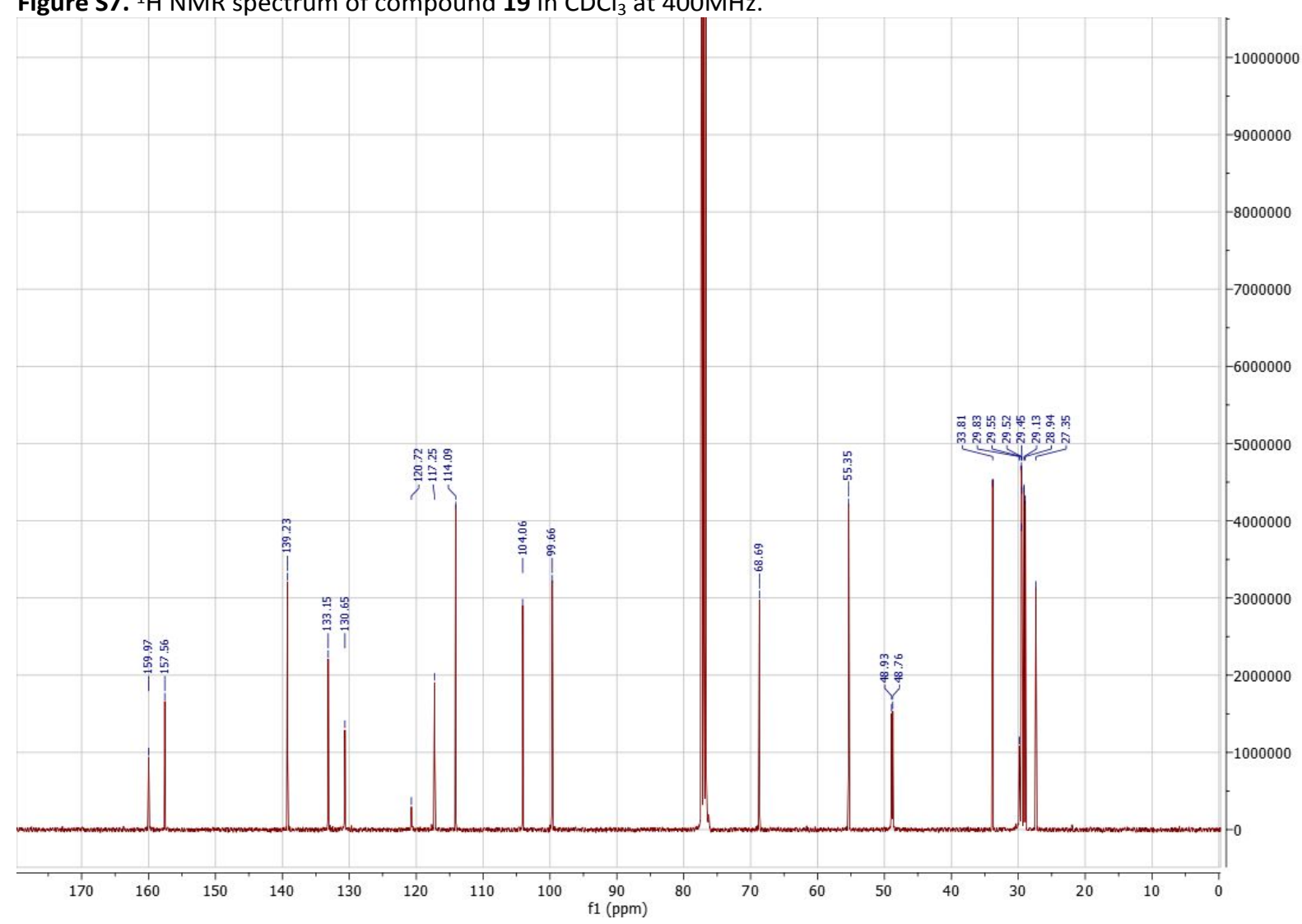

Figure S8. ${ }^{13} \mathrm{C}$ NMR spectrum of compound 19 in $\mathrm{CDCl}_{3}$ at $400 \mathrm{MHz}$. 


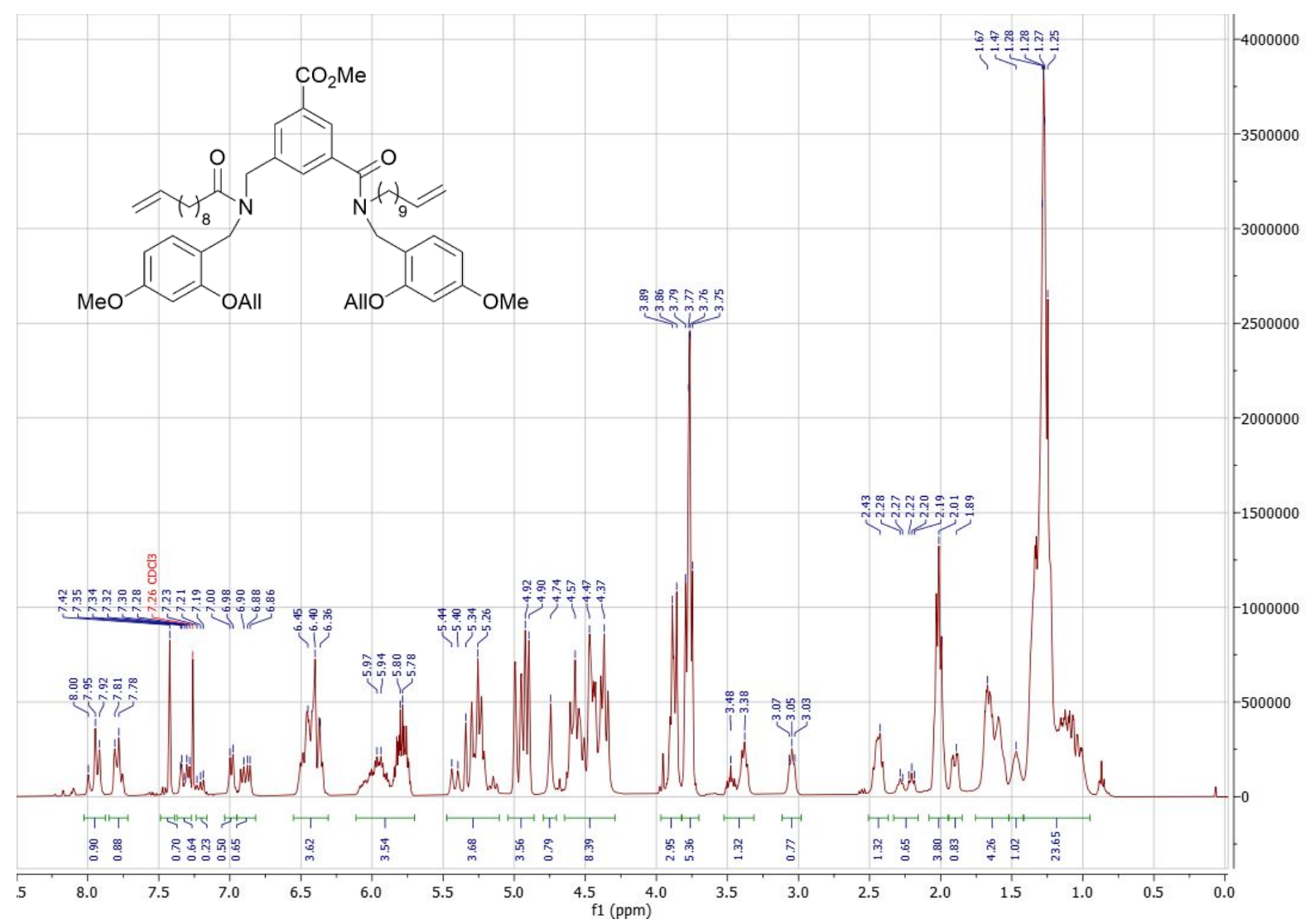

Figure S9. ${ }^{1} \mathrm{H}$ NMR spectrum of compound 6 in $\mathrm{CDCl}_{3}$ at $400 \mathrm{MHz}$.

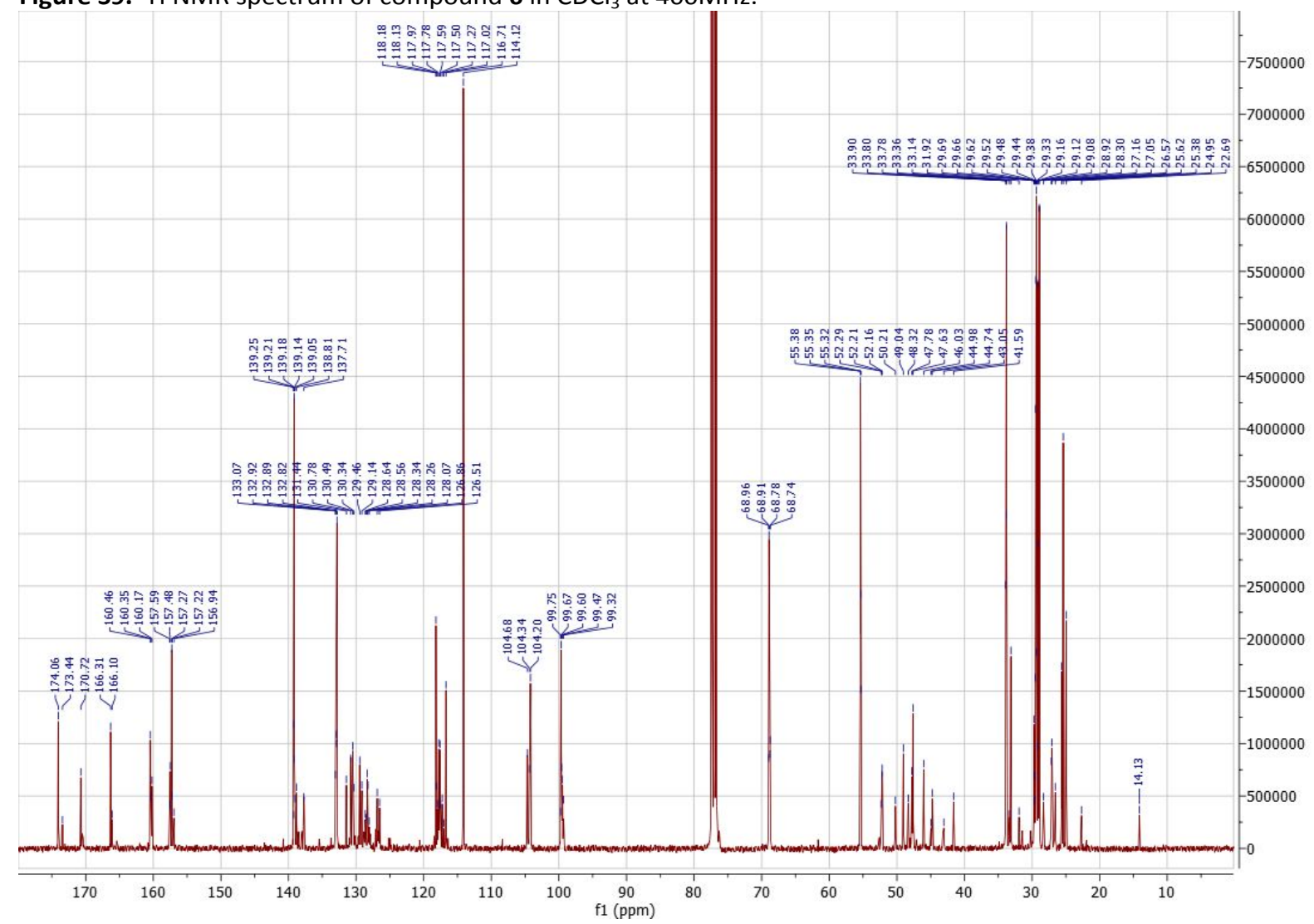

Figure S10. ${ }^{13} \mathrm{C}$ NMR spectrum of compound 6 in $\mathrm{CDCl}_{3}$ at $400 \mathrm{MHz}$. 


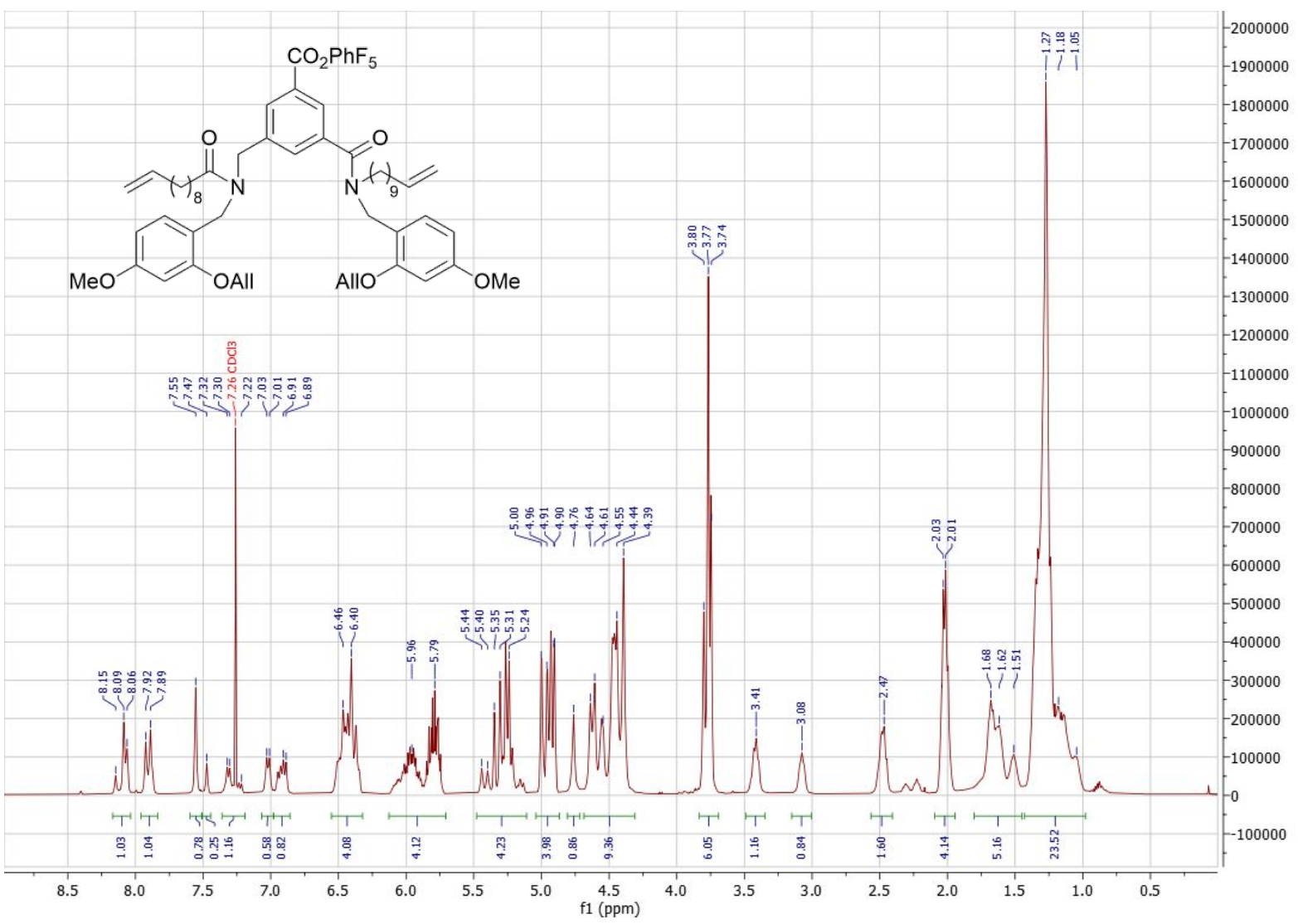

Figure S11. ${ }^{1} \mathrm{H}$ NMR spectrum of compound 20 in $\mathrm{CDCl}_{3}$ at $400 \mathrm{MHz}$.

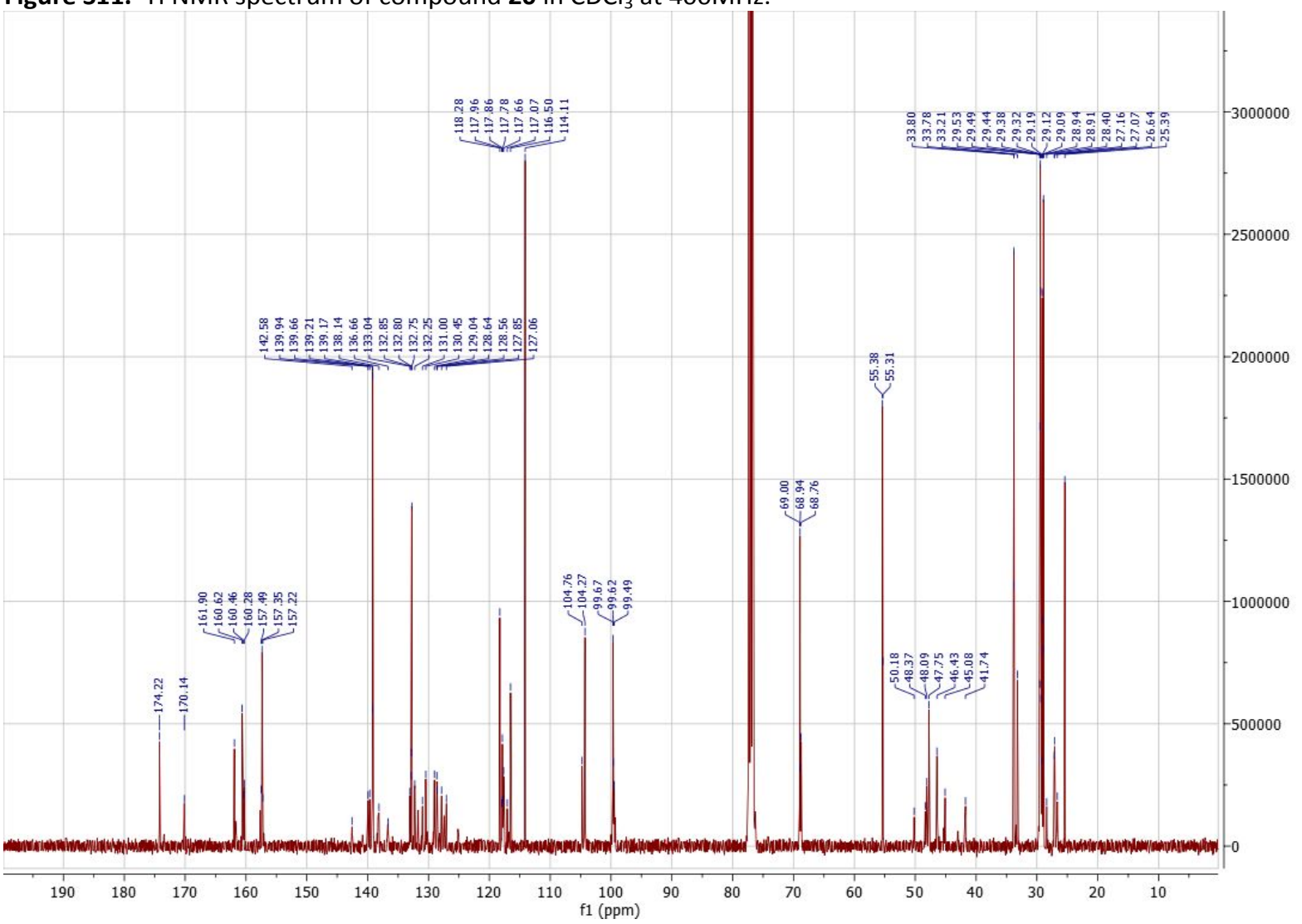

Figure S12. ${ }^{13} \mathrm{C}$ NMR spectrum of compound 20 in $\mathrm{CDCl}_{3}$ at $400 \mathrm{MHz}$. 


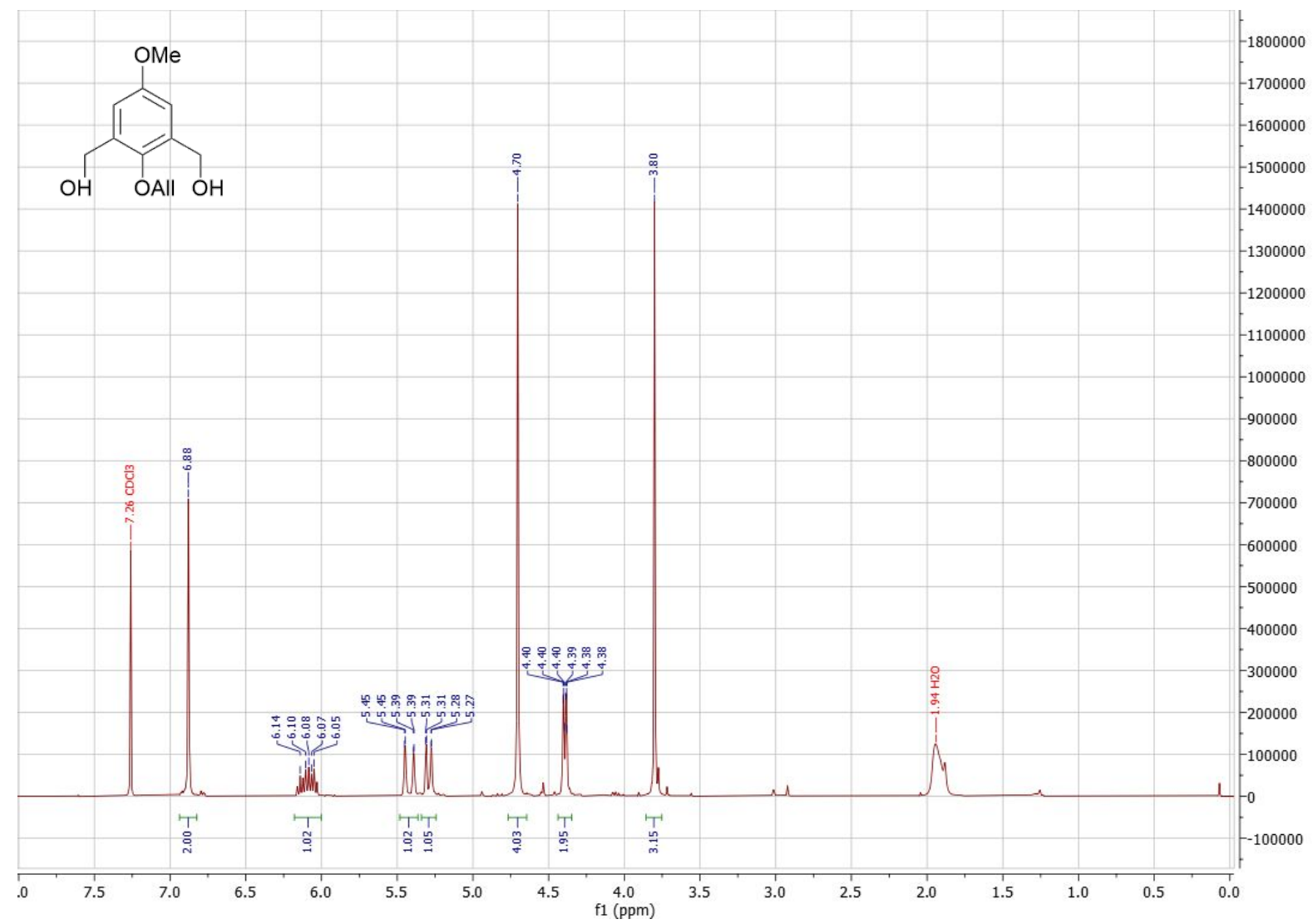

Figure S13. ${ }^{1} \mathrm{H}$ NMR spectrum of compound 21 in $\mathrm{CDCl}_{3}$ at $300 \mathrm{MHz}$.

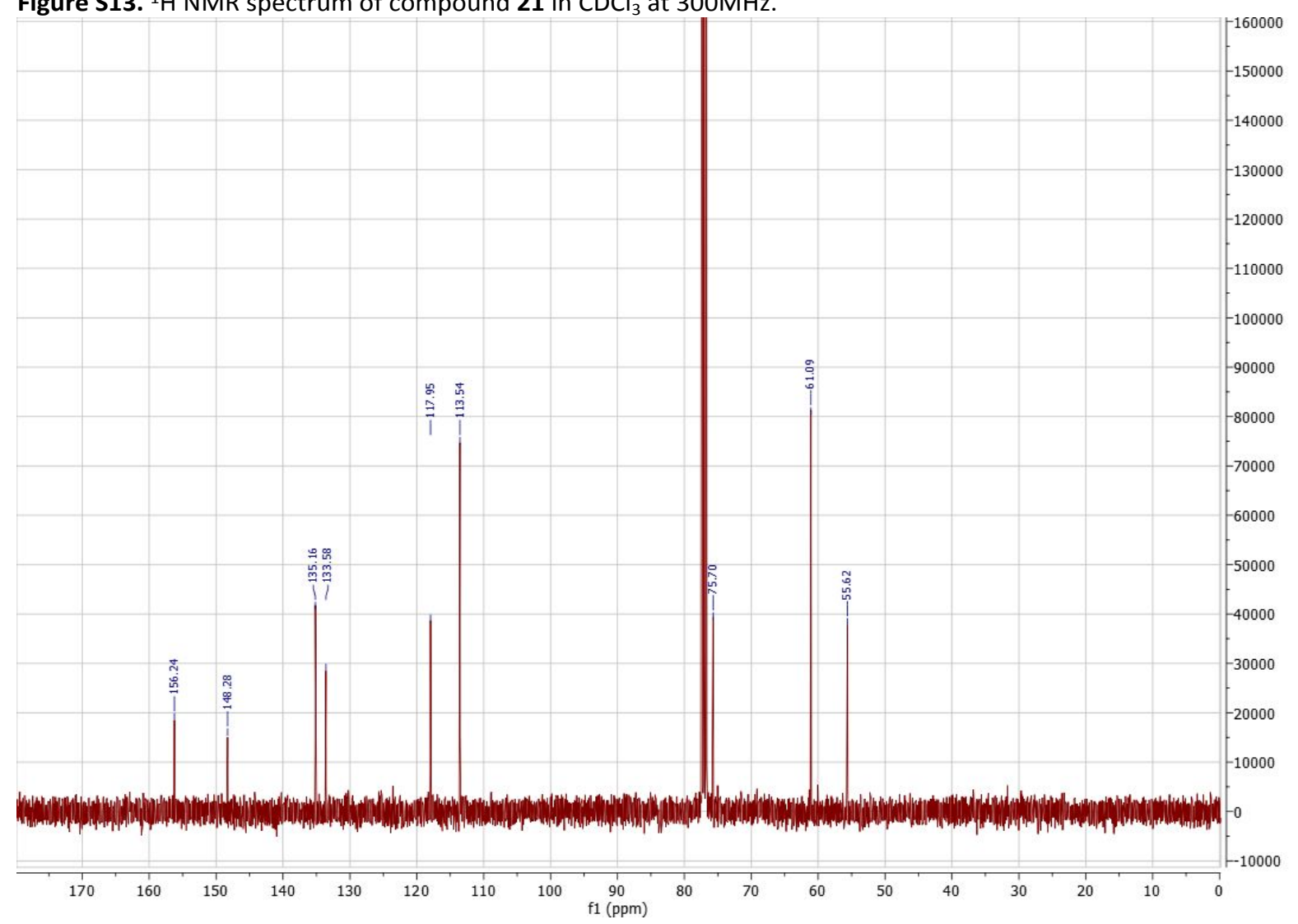

Figure S14. ${ }^{13} \mathrm{C}$ NMR spectrum of compound 21 in $\mathrm{CDCl}_{3}$ at $300 \mathrm{MHz}$. 


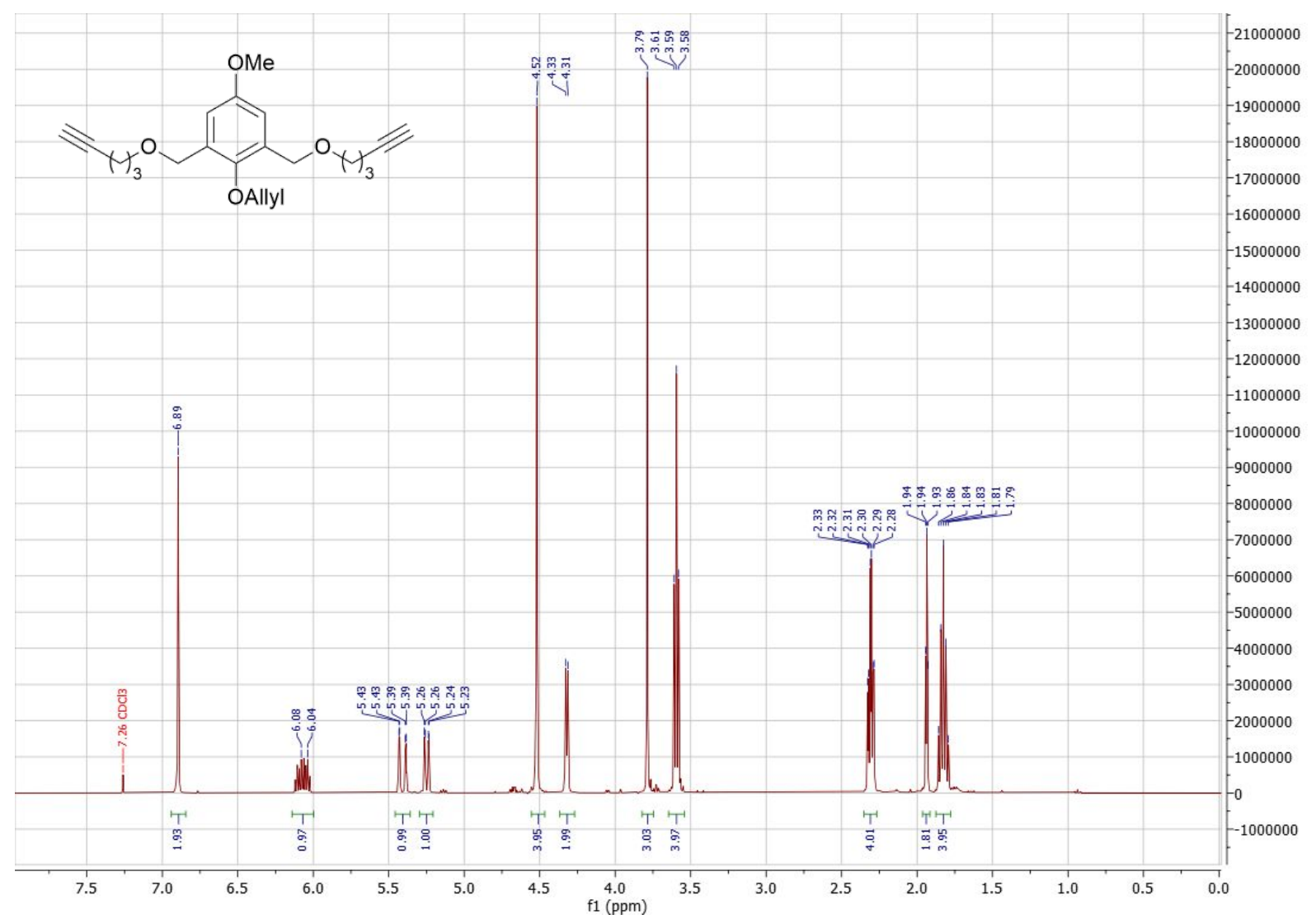

Figure S15. ${ }^{1} \mathrm{H}$ NMR spectrum of compound 22 in $\mathrm{CDCl}_{3}$ at $400 \mathrm{MHz}$.

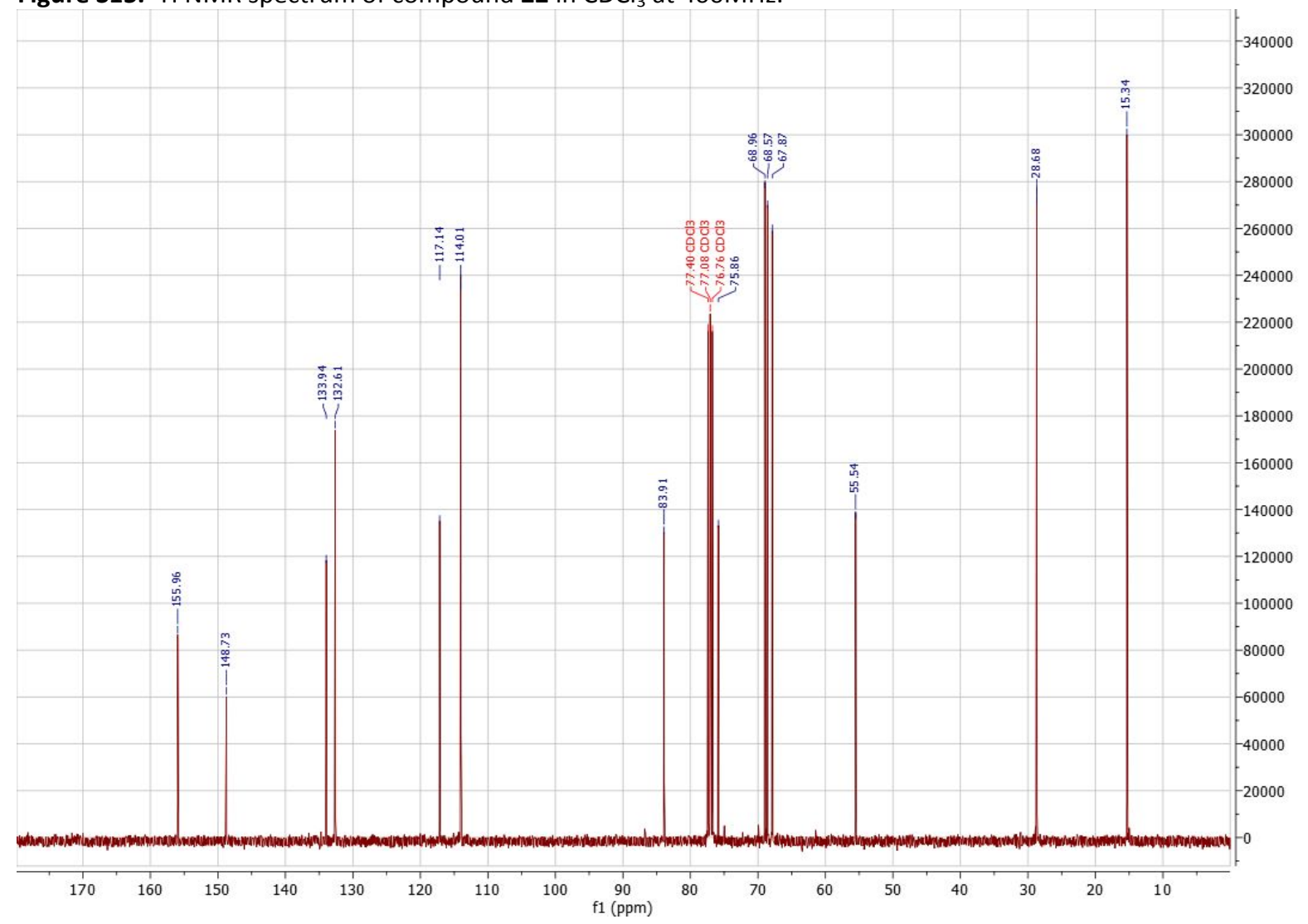

Figure S16. ${ }^{13} \mathrm{C}$ NMR spectrum of compound 22 in $\mathrm{CDCl}_{3}$ at $400 \mathrm{MHz}$. 


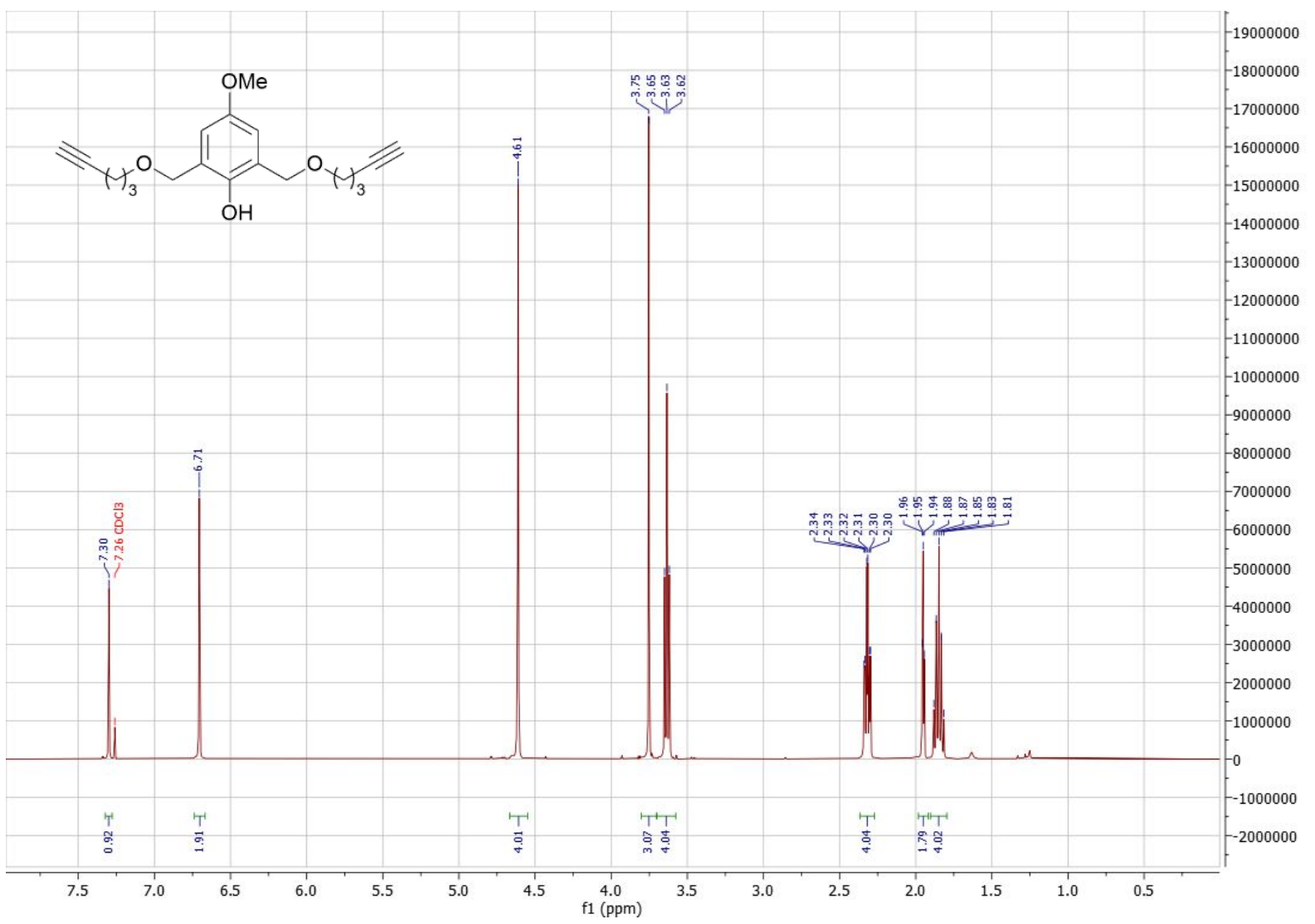

Figure S17. ${ }^{1} \mathrm{H}$ NMR spectrum of compound $\mathbf{5}$ in $\mathrm{CDCl}_{3}$ at $400 \mathrm{MHz}$.

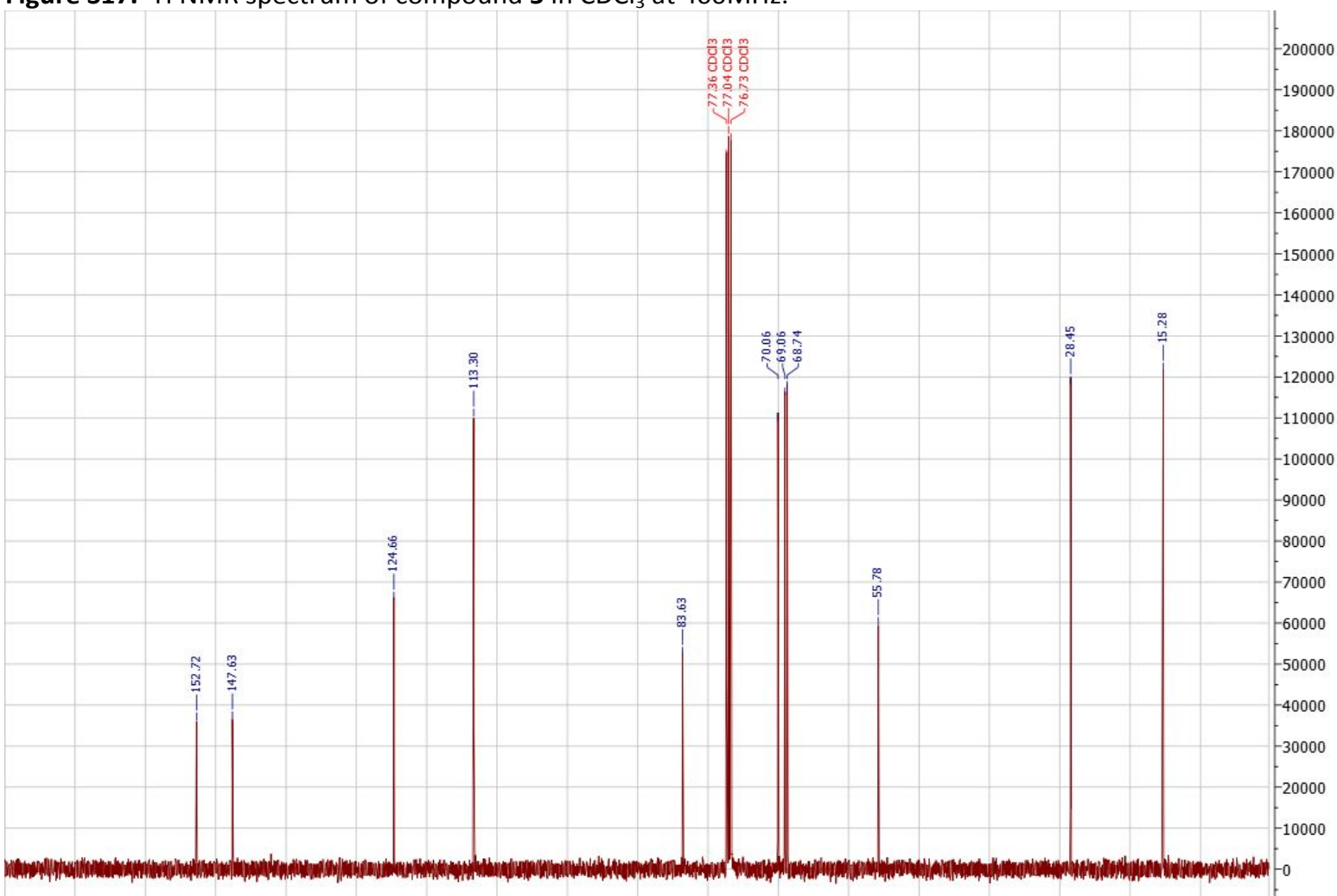

\begin{tabular}{llllllllllllllllll}
\hline 0 & 170 & 160 & 150 & 140 & 130 & 120 & 110 & 100 & $\begin{array}{c}1 \\
\mathrm{f} 1(\mathrm{ppm})\end{array}$ & 80 & 70 & 60 & 50 & 40 & 30 & 20 & 10
\end{tabular}

Figure S18. ${ }^{13} \mathrm{C}$ NMR spectrum of compound 5 in $\mathrm{CDCl}_{3}$ at $400 \mathrm{MHz}$. 


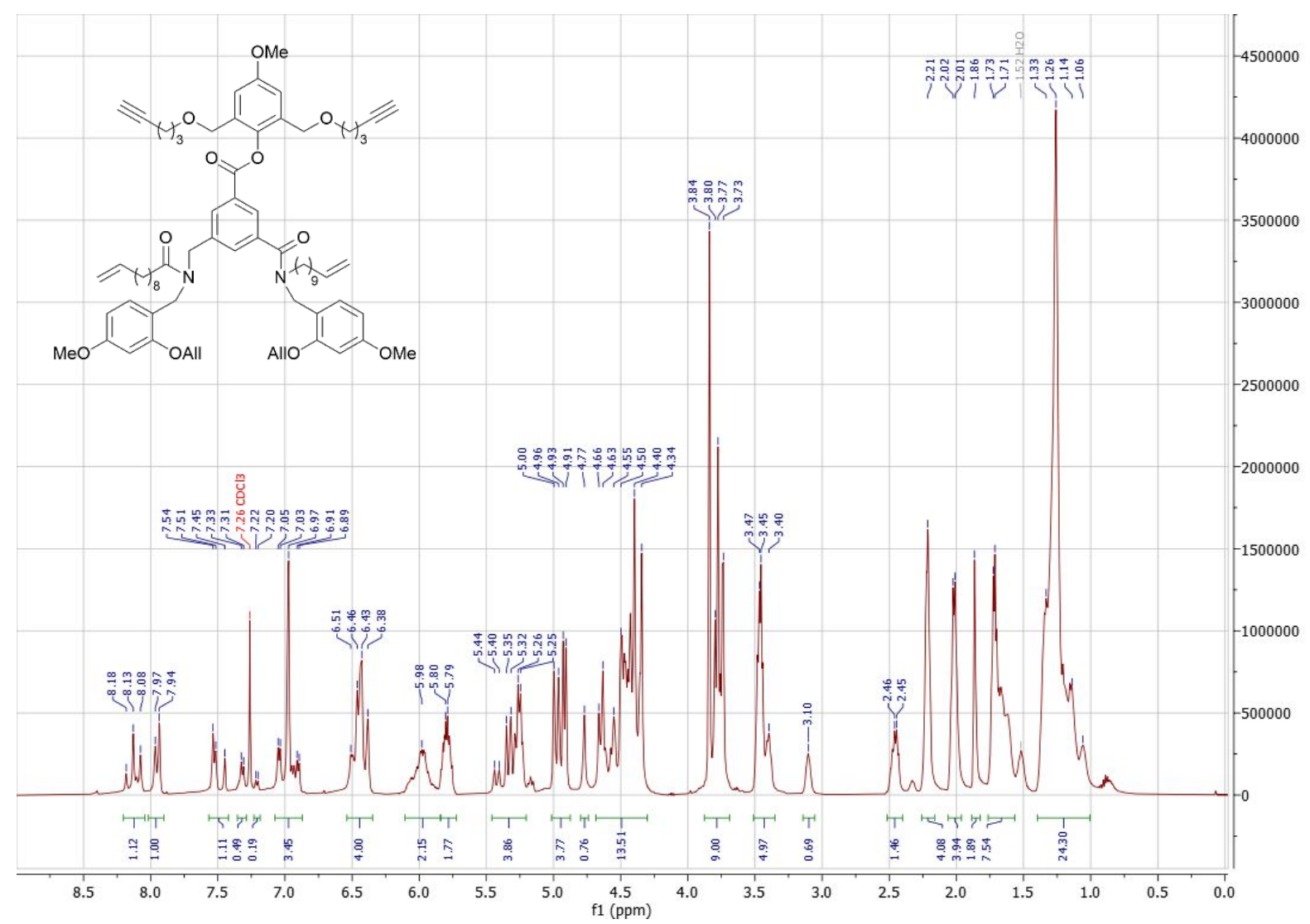

Figure S19. ${ }^{1} \mathrm{H}$ NMR spectrum of compound 23 in $\mathrm{CDCl}_{3}$ at $500 \mathrm{MHz}$.

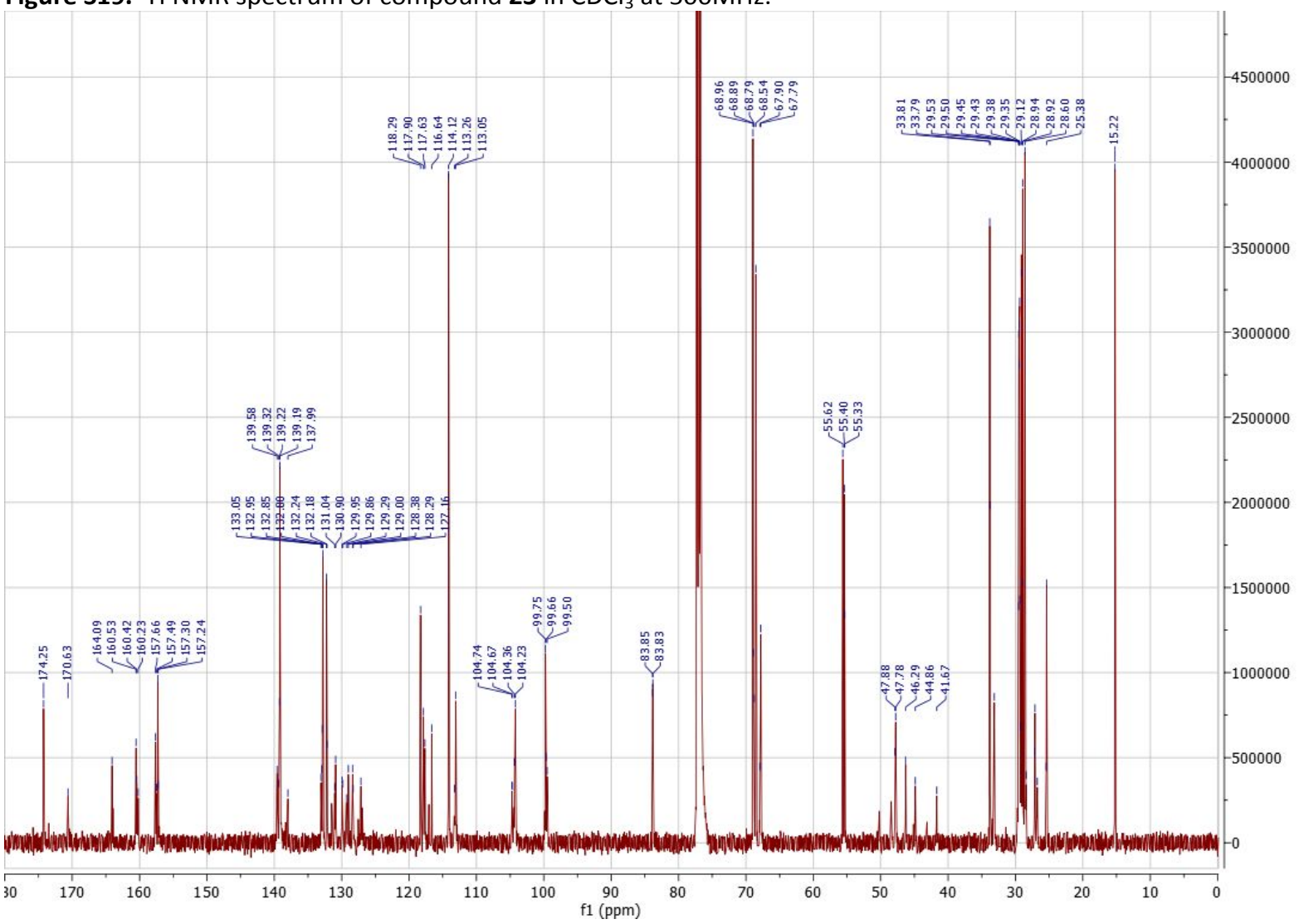

Figure S20. ${ }^{13} \mathrm{C}$ NMR spectrum of compound 23 in $\mathrm{CDCl}_{3}$ at $500 \mathrm{MHz}$. 


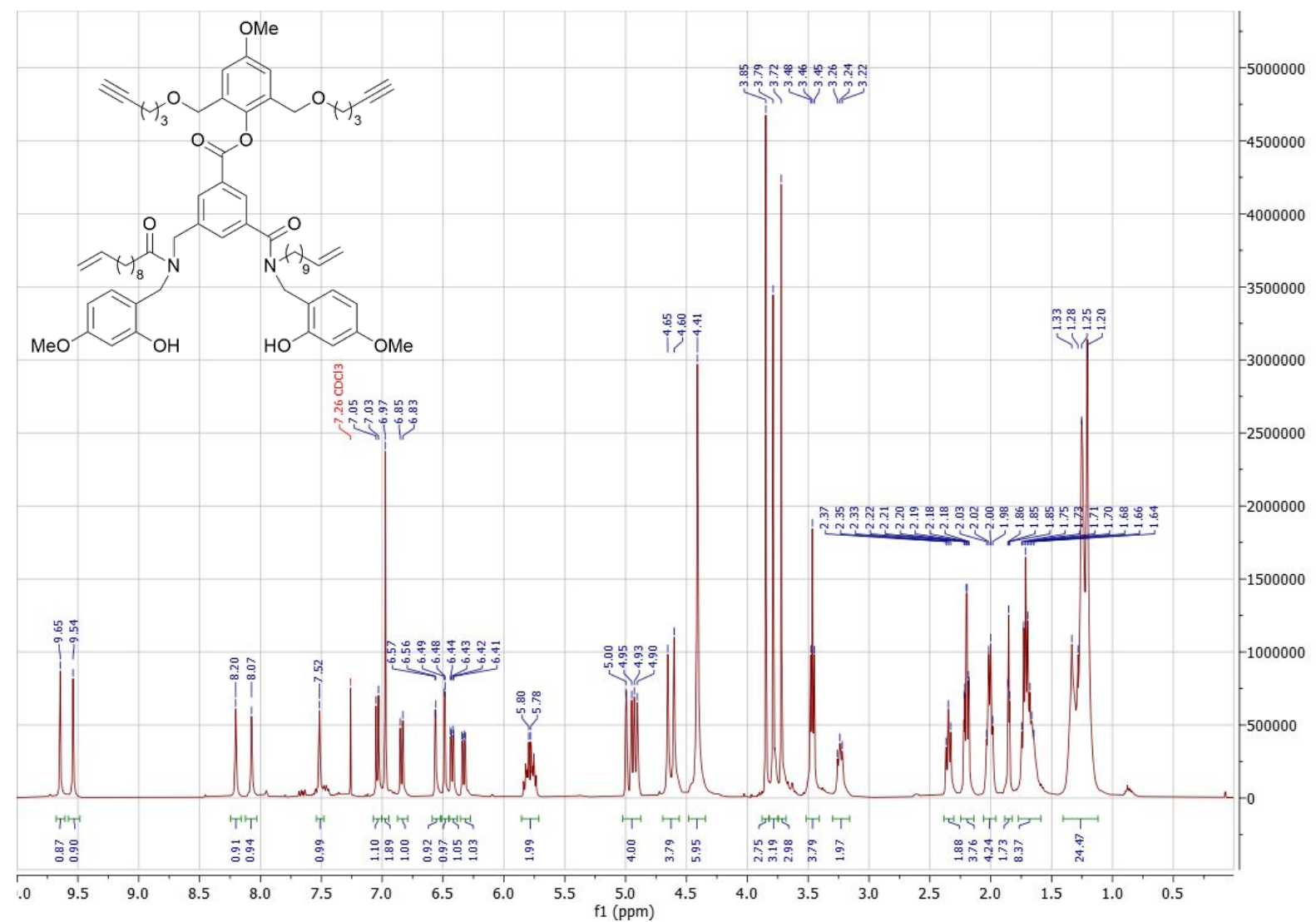

Figure S21. ${ }^{1} \mathrm{H}$ NMR spectrum of compound 7 in $\mathrm{CDCl}_{3}$ at $400 \mathrm{MHz}$.

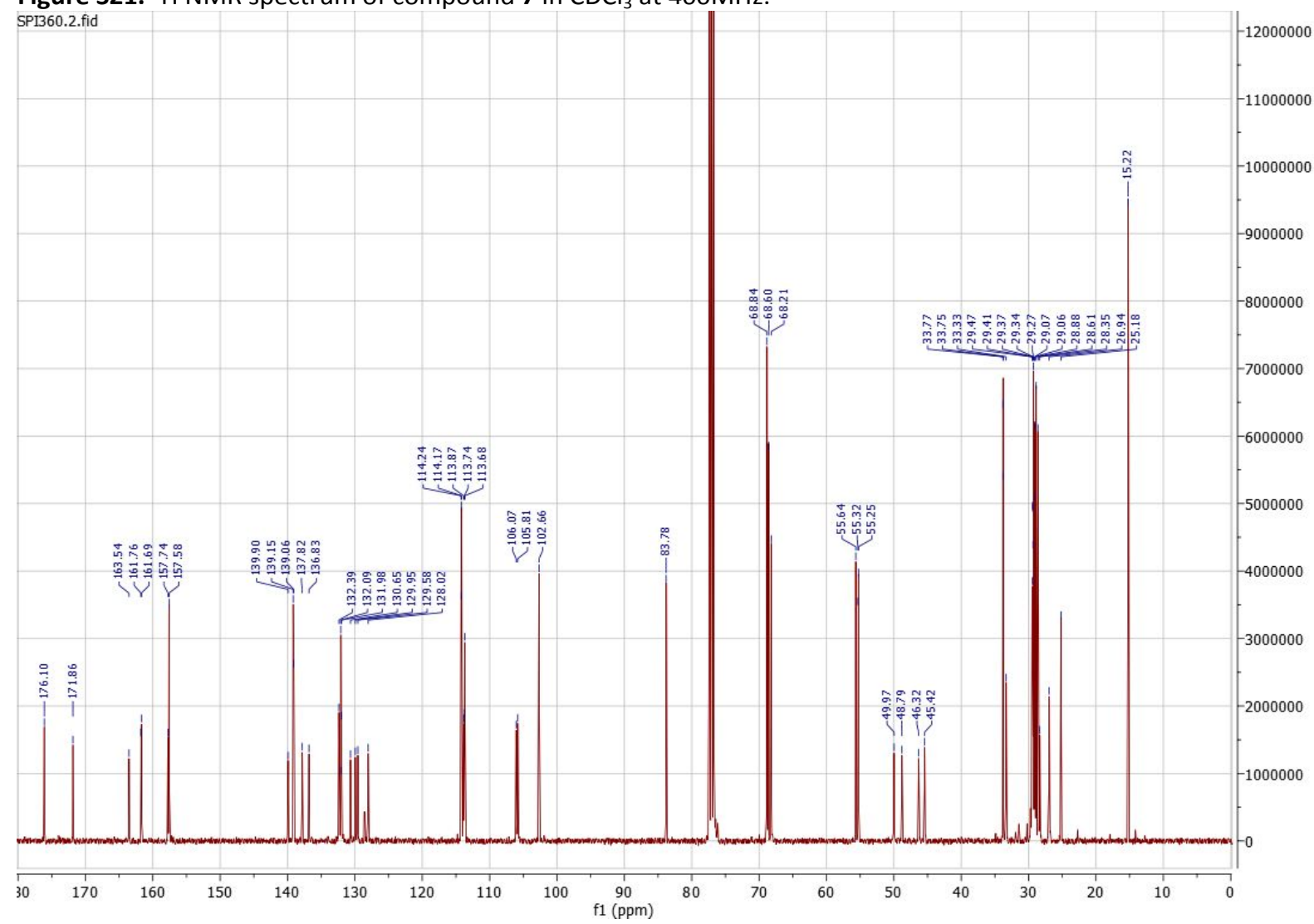

Figure S22. ${ }^{13} \mathrm{C}$ NMR spectrum of compound $\mathbf{7}$ in $\mathrm{CDCl}_{3}$ at $400 \mathrm{MHz}$. 


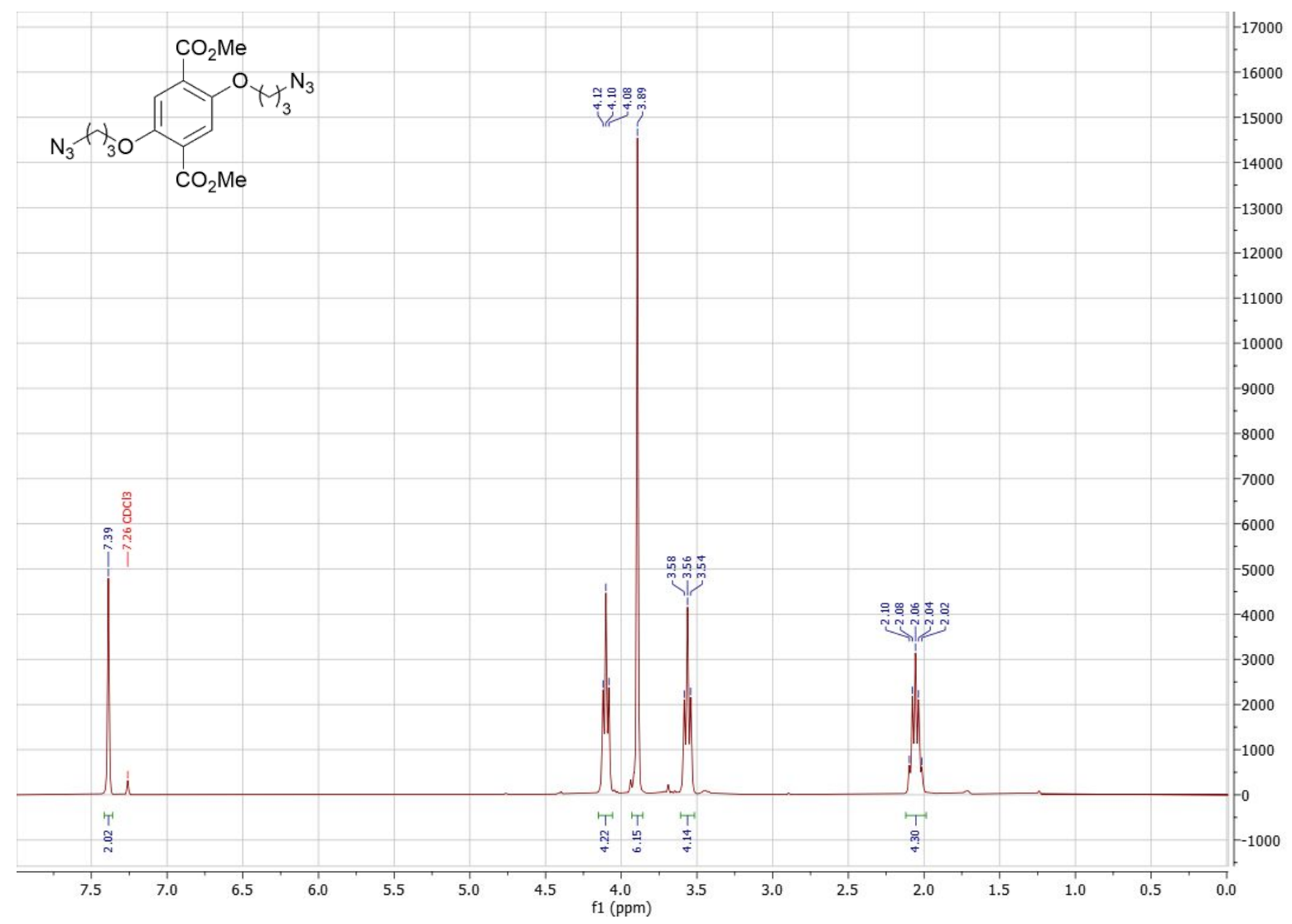

Figure S23. ${ }^{1} \mathrm{H}$ NMR spectrum of compound 24 in $\mathrm{CDCl}_{3}$ at $300 \mathrm{MHz}$.

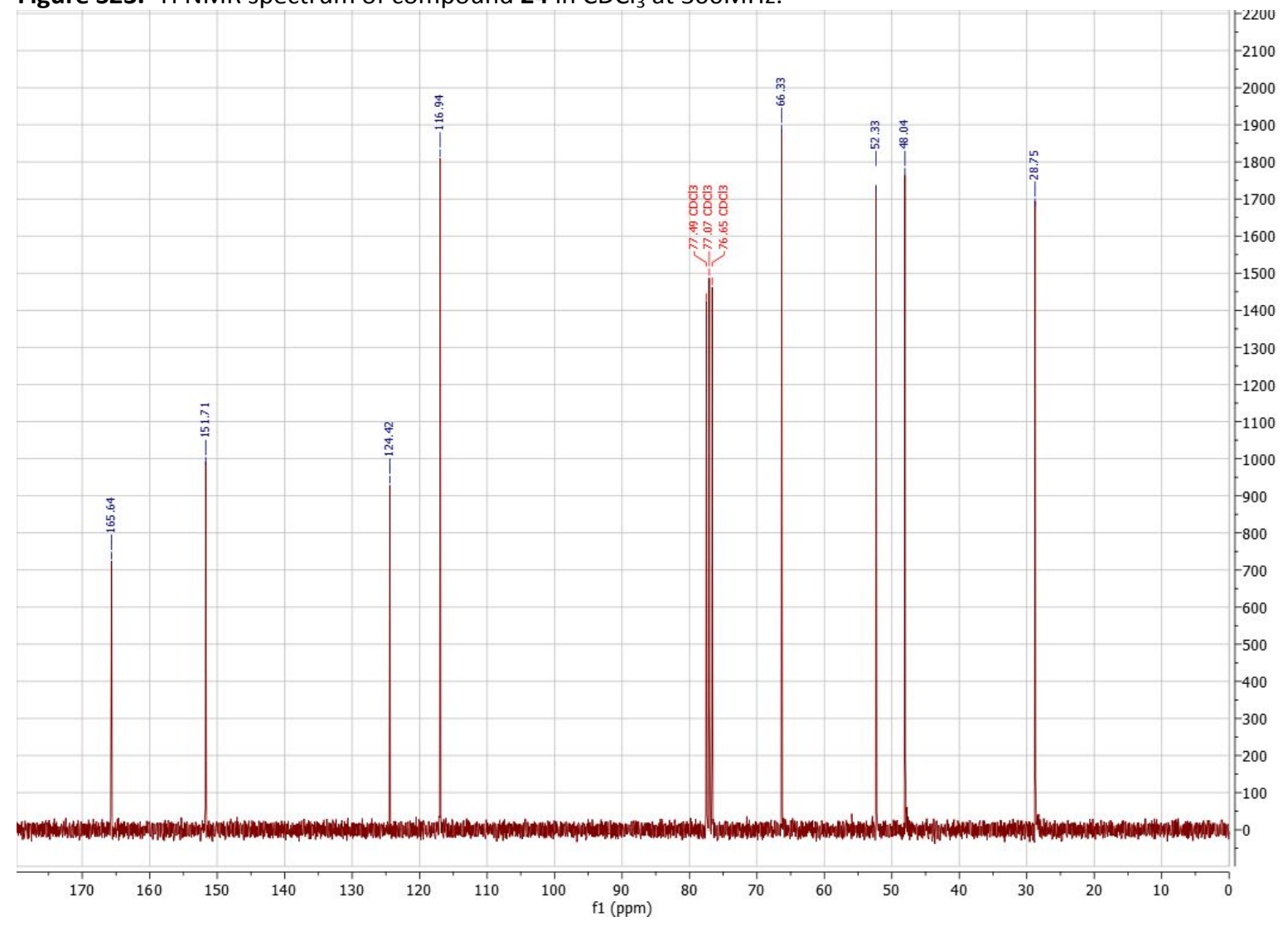

Figure S24. ${ }^{13} \mathrm{C}$ NMR spectrum of compound 24 in $\mathrm{CDCl}_{3}$ at $300 \mathrm{MHz}$. 


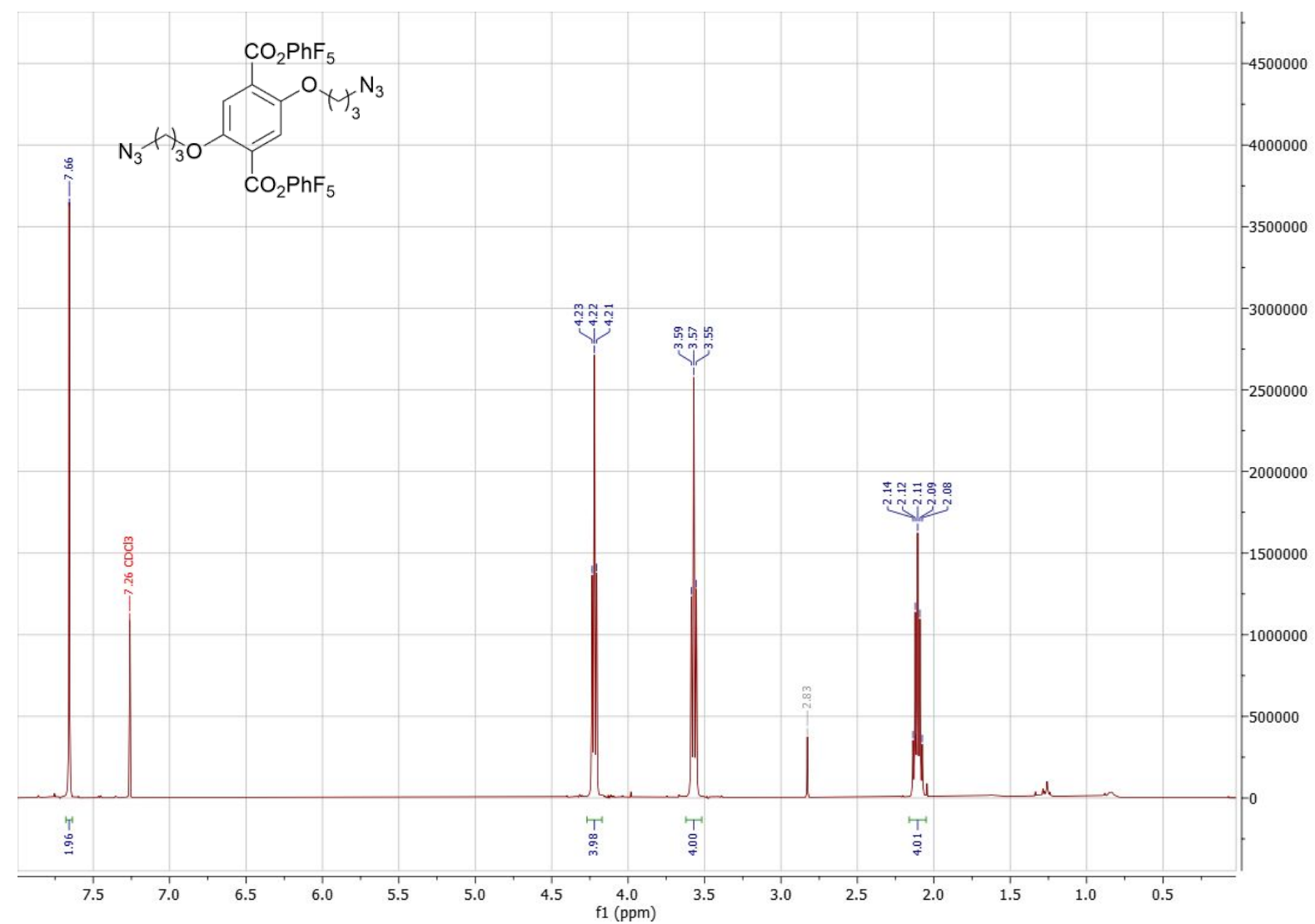

Figure S25. ${ }^{1} \mathrm{H}$ NMR spectrum of compound 4 in $\mathrm{CDCl}_{3}$ at $400 \mathrm{MHz}$.

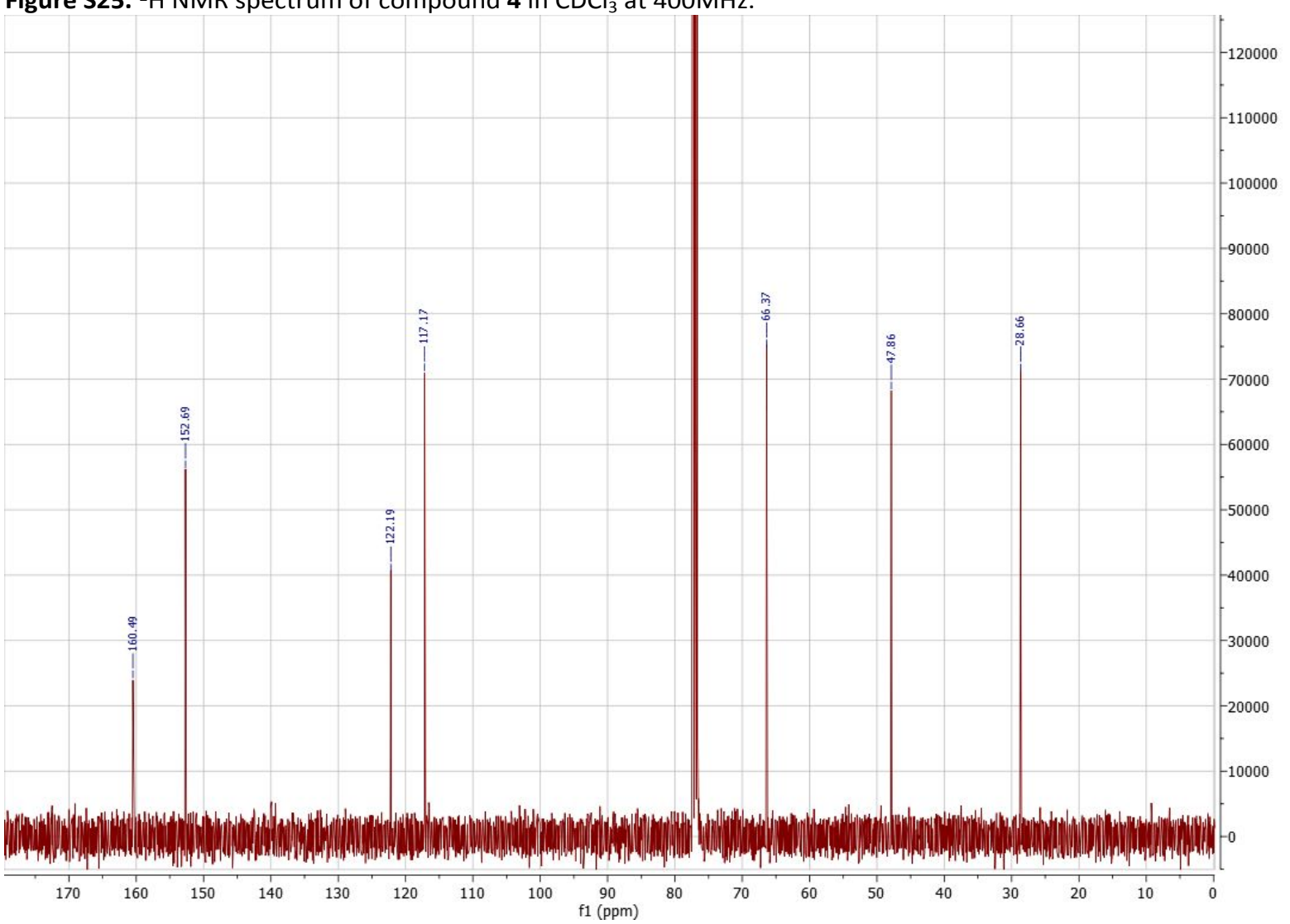

Figure S26. ${ }^{13} \mathrm{C}$ NMR spectrum of compound 4 in $\mathrm{CDCl}_{3}$ at $400 \mathrm{MHz}$. 


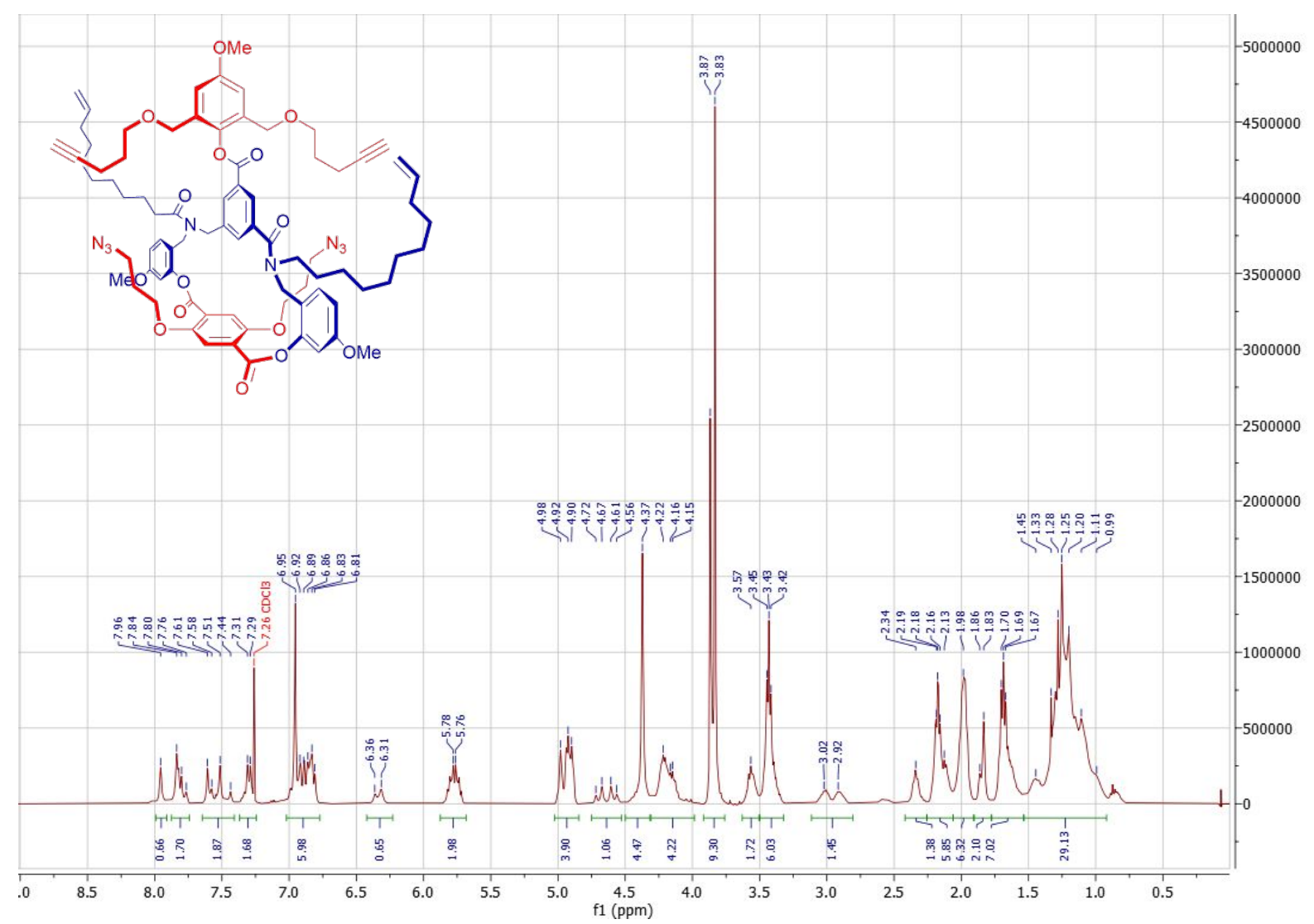

Figure S27. ${ }^{1} \mathrm{H}$ NMR spectrum of compound 8 in $\mathrm{CDCl}_{3}$ at $400 \mathrm{MHz}$.

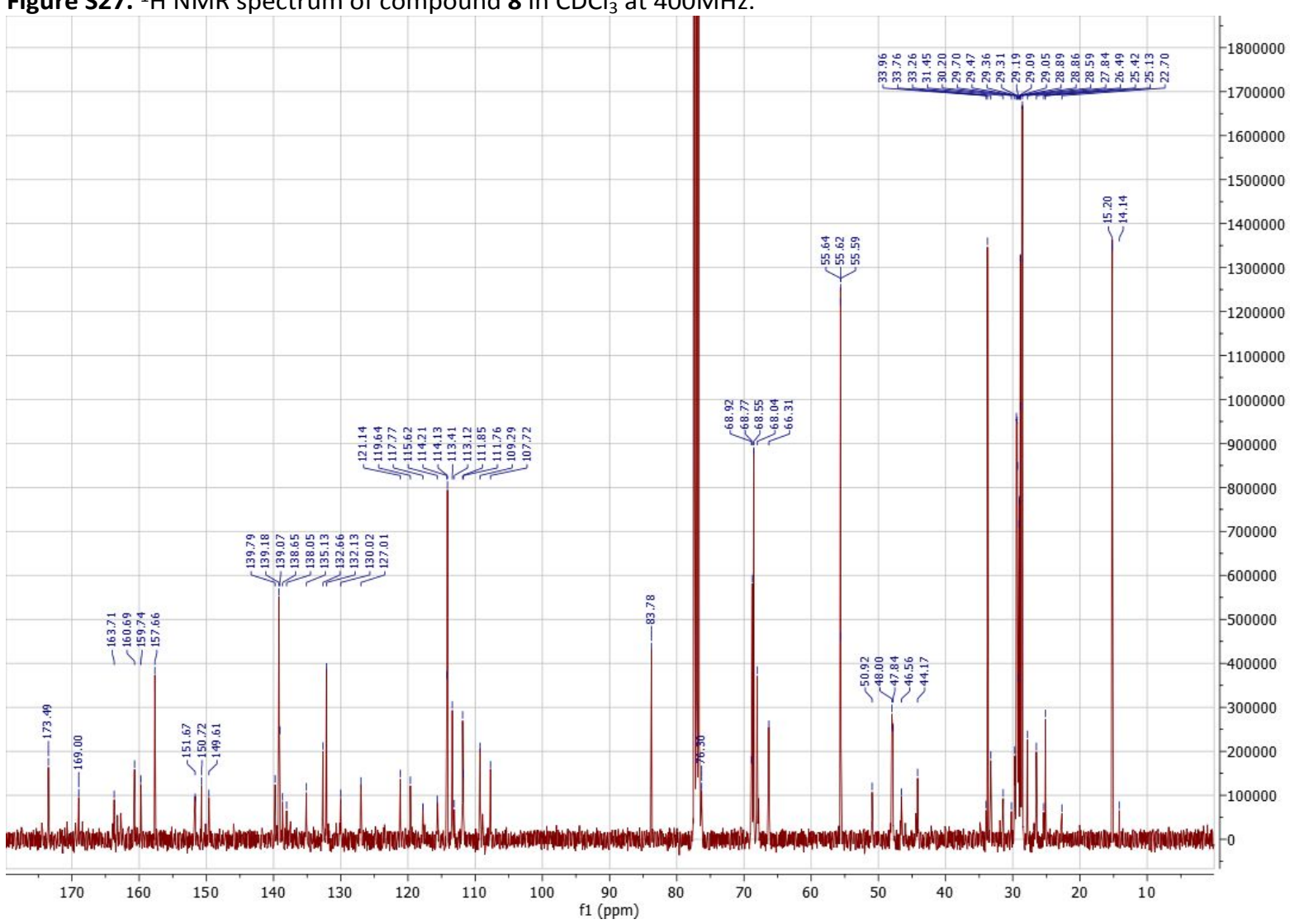

Figure S28. ${ }^{13} \mathrm{C}$ NMR spectrum of compound 8 in $\mathrm{CDCl}_{3}$ at $400 \mathrm{MHz}$. 


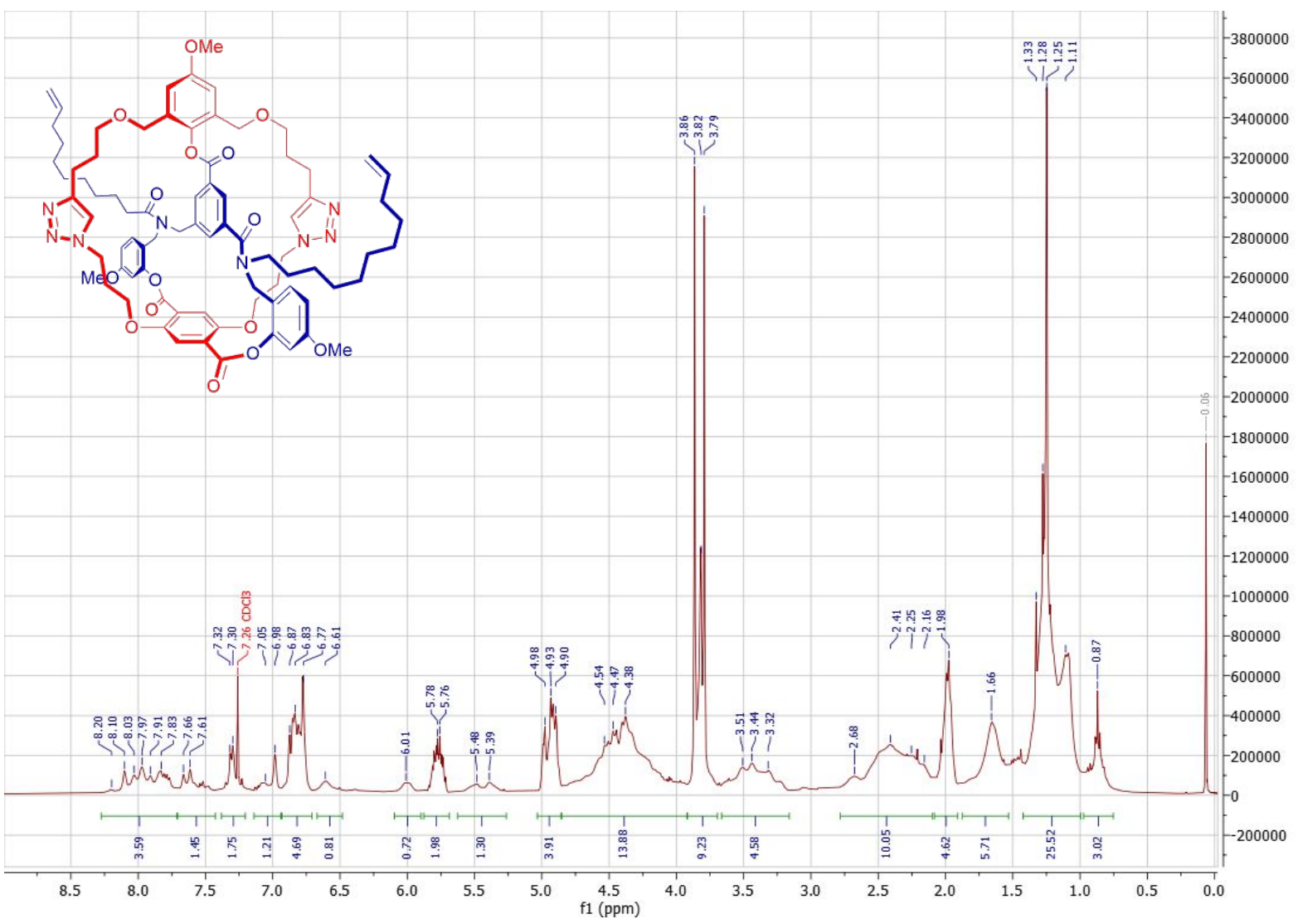

Figure S29. ${ }^{1} \mathrm{H}$ NMR spectrum of compound 1 in $\mathrm{CDCl}_{3}$ at $400 \mathrm{MHz}$.

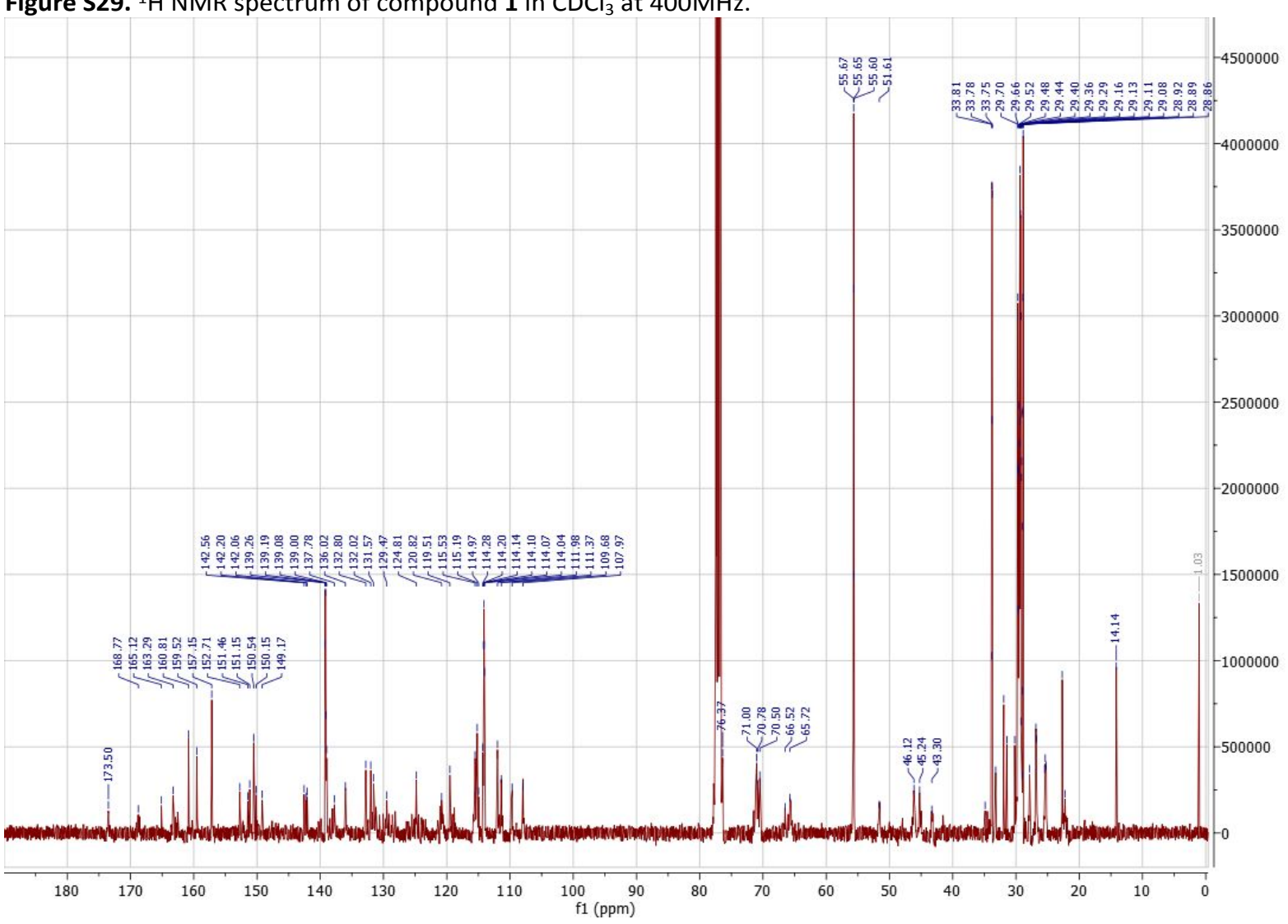

Figure S30. ${ }^{13} \mathrm{C}$ NMR spectrum of compound 1 in $\mathrm{CDCl}_{3}$ at $400 \mathrm{MHz}$. 


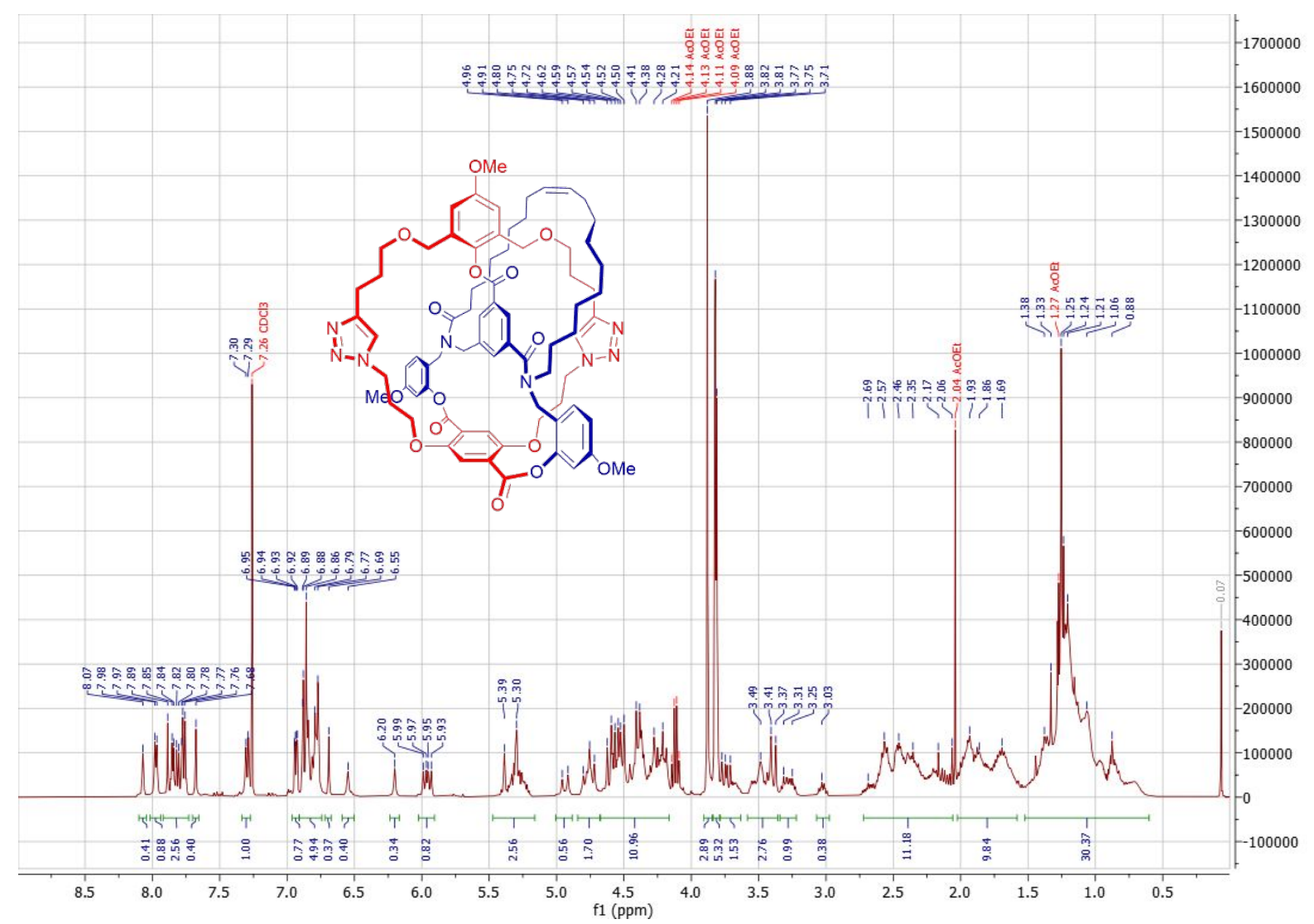

Figure S31. ${ }^{1} \mathrm{H}$ NMR spectrum of compound 25 in $\mathrm{CDCl}_{3}$ at $400 \mathrm{MHz}$.

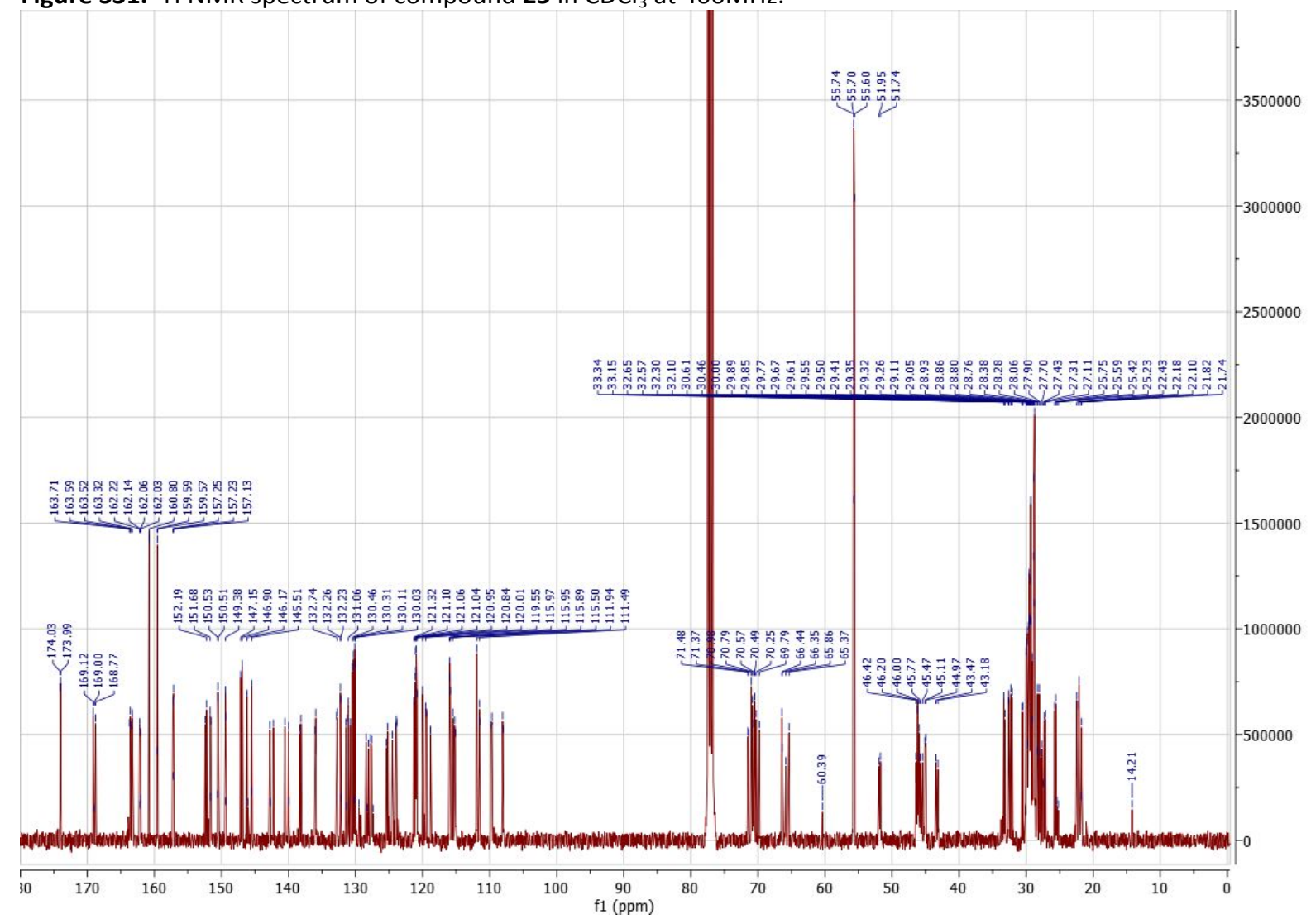

Figure S32. ${ }^{13} \mathrm{C}$ NMR spectrum of compound 25 in $\mathrm{CDCl}_{3}$ at $400 \mathrm{MHz}$. 


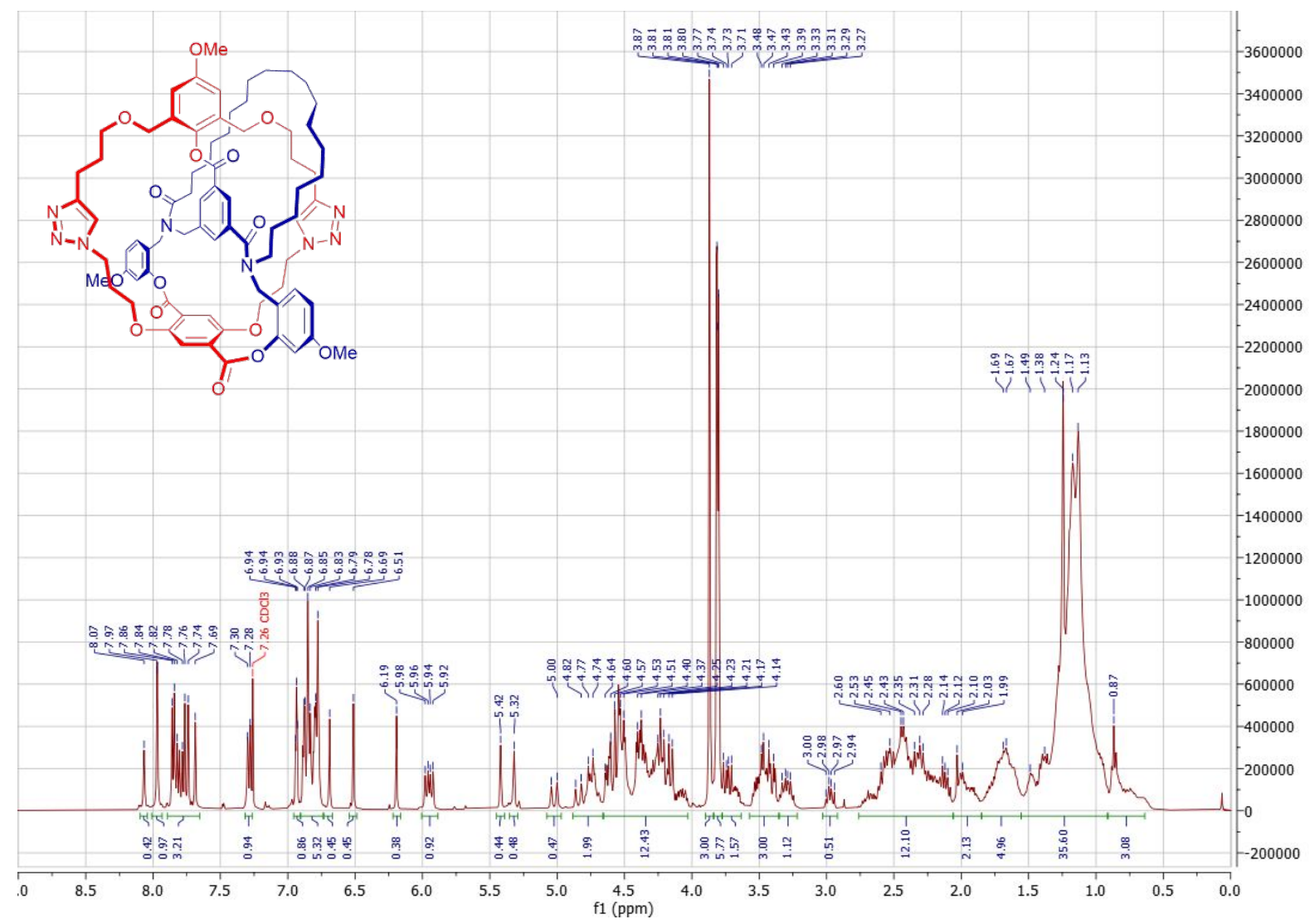

Figure S33. ${ }^{1} \mathrm{H}$ NMR spectrum of compound 9 in $\mathrm{CDCl}_{3}$ at $400 \mathrm{MHz}$.

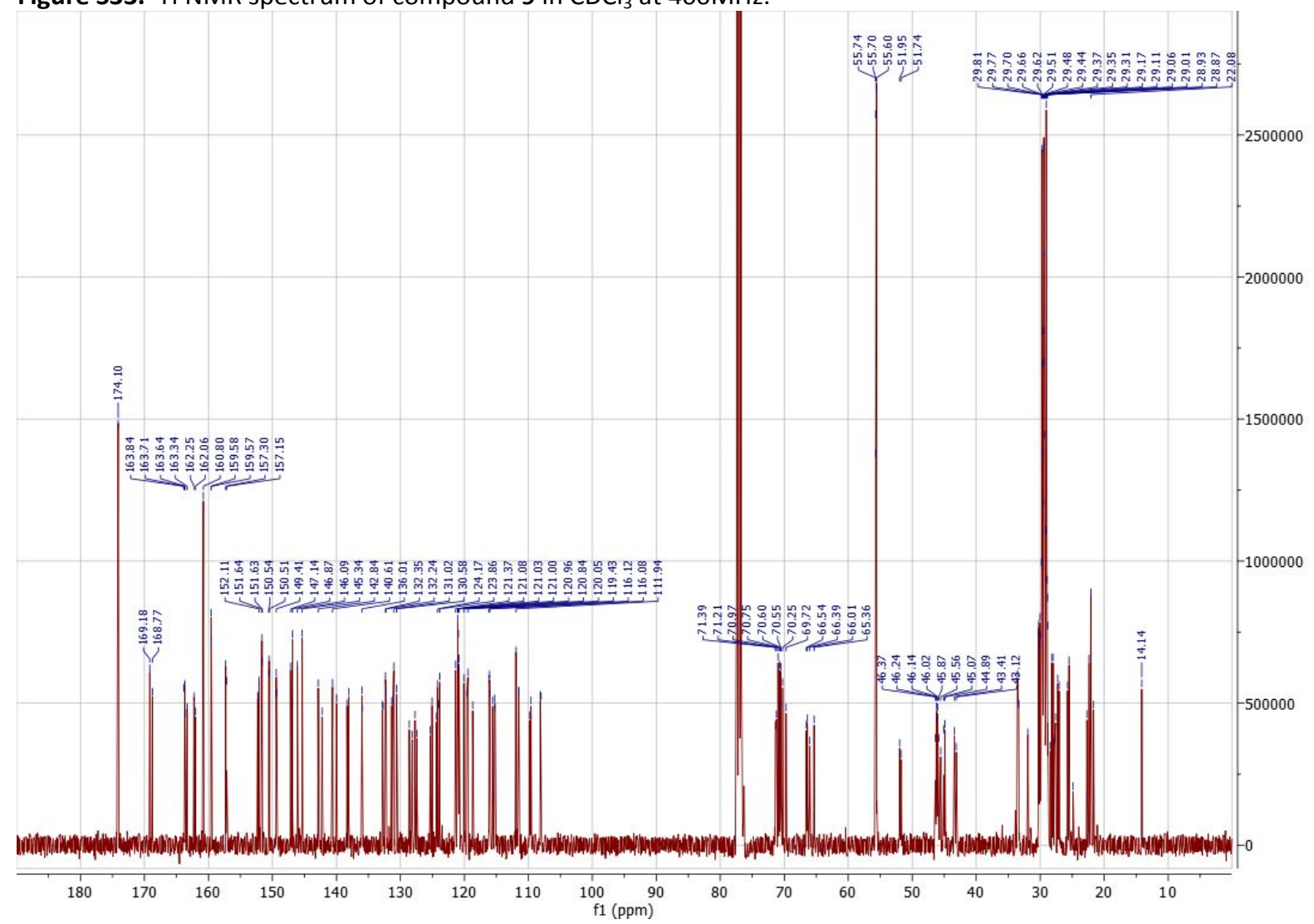

Figure S34. ${ }^{13} \mathrm{C}$ NMR spectrum of compound 9 in $\mathrm{CDCl}_{3}$ at $400 \mathrm{MHz}$. 


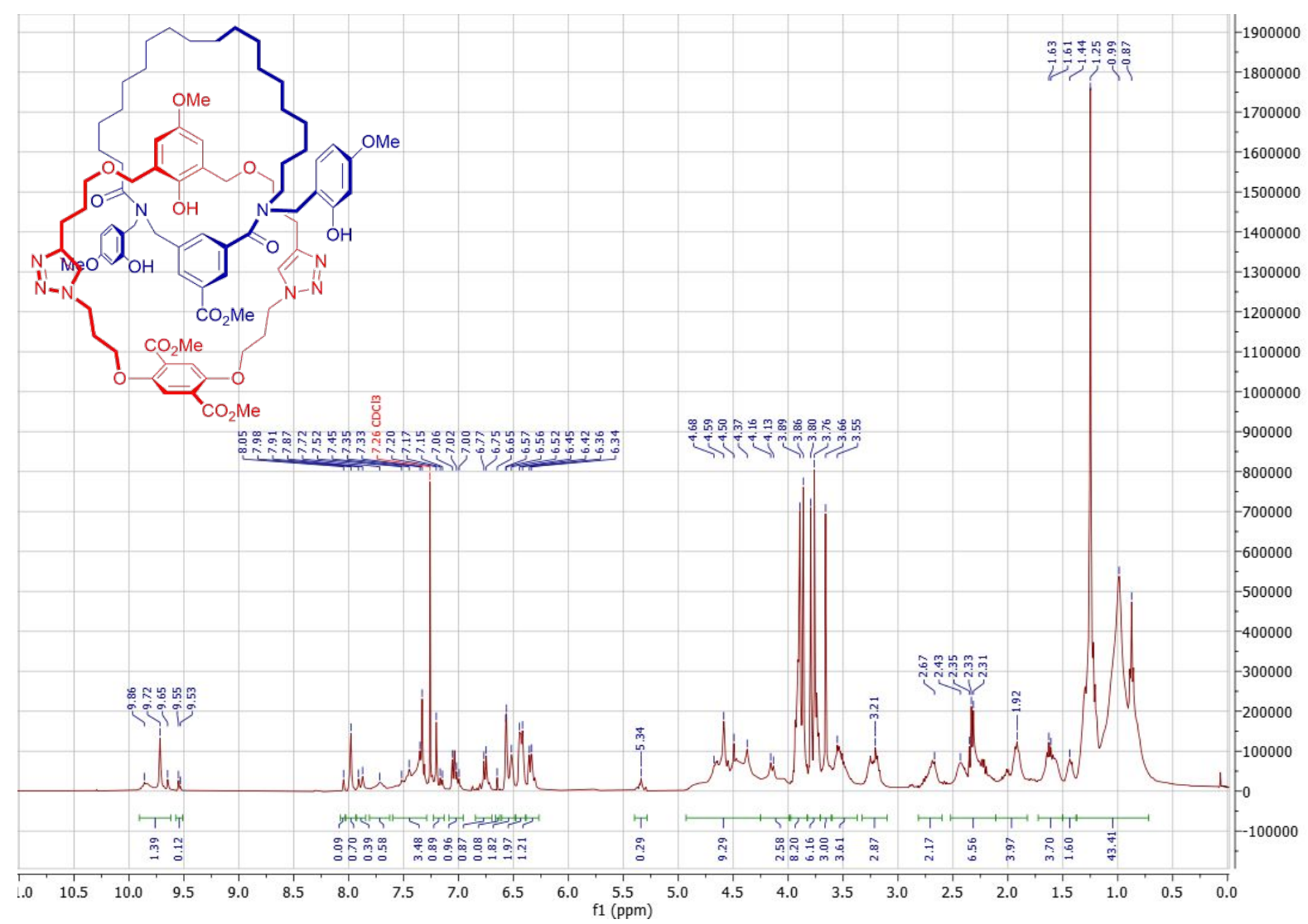

Figure S35. ${ }^{1} \mathrm{H}$ NMR spectrum of compound 10 in $\mathrm{CDCl}_{3}$ at $400 \mathrm{MHz}$.

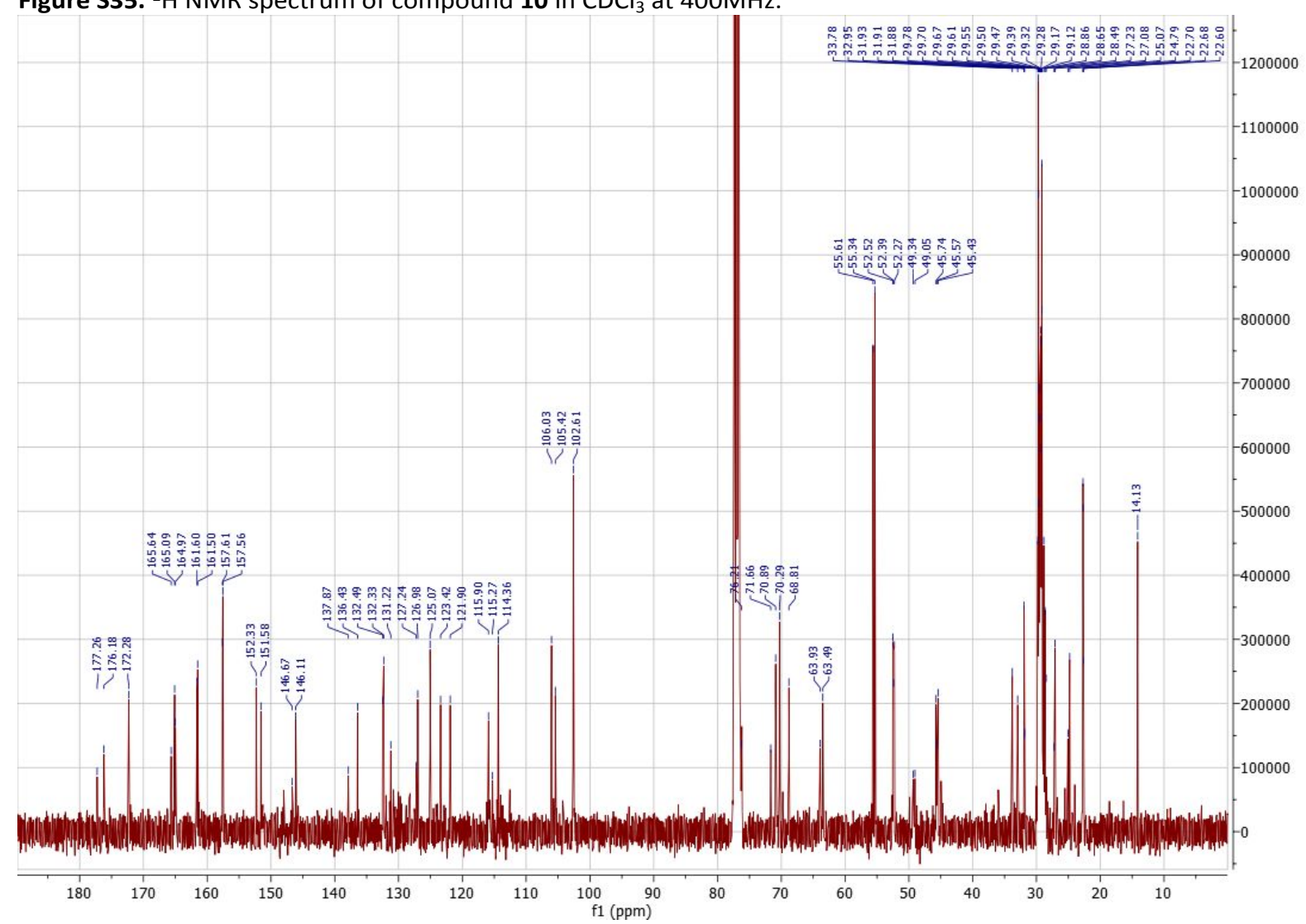

Figure S36. ${ }^{13} \mathrm{C}$ NMR spectrum of compound 10 in $\mathrm{CDCl}_{3}$ at $400 \mathrm{MHz}$. 


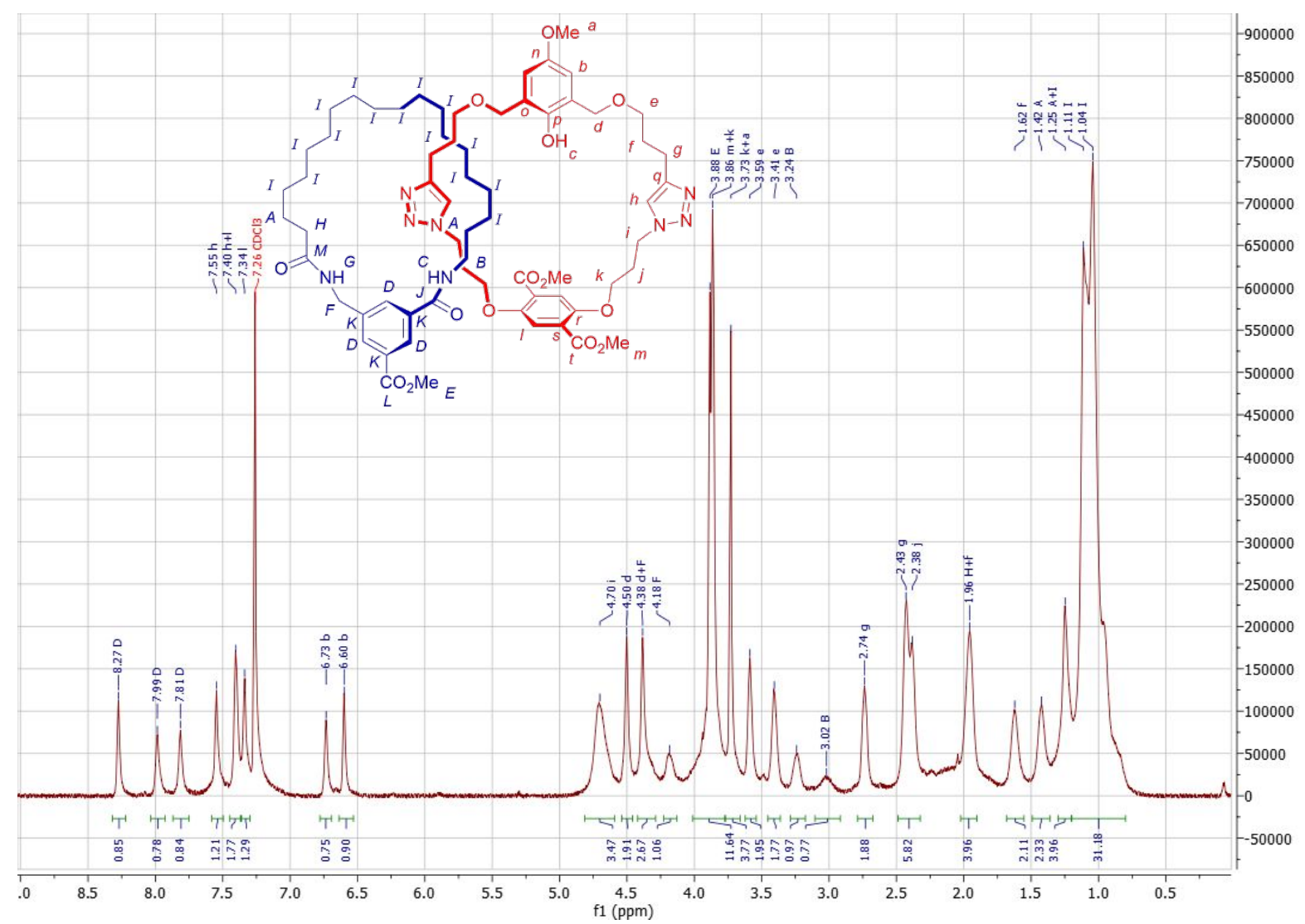

Figure S37. ${ }^{1} \mathrm{H}$ NMR spectrum of compound 2 in $\mathrm{CDCl}_{3}$ at $500 \mathrm{MHz}$.

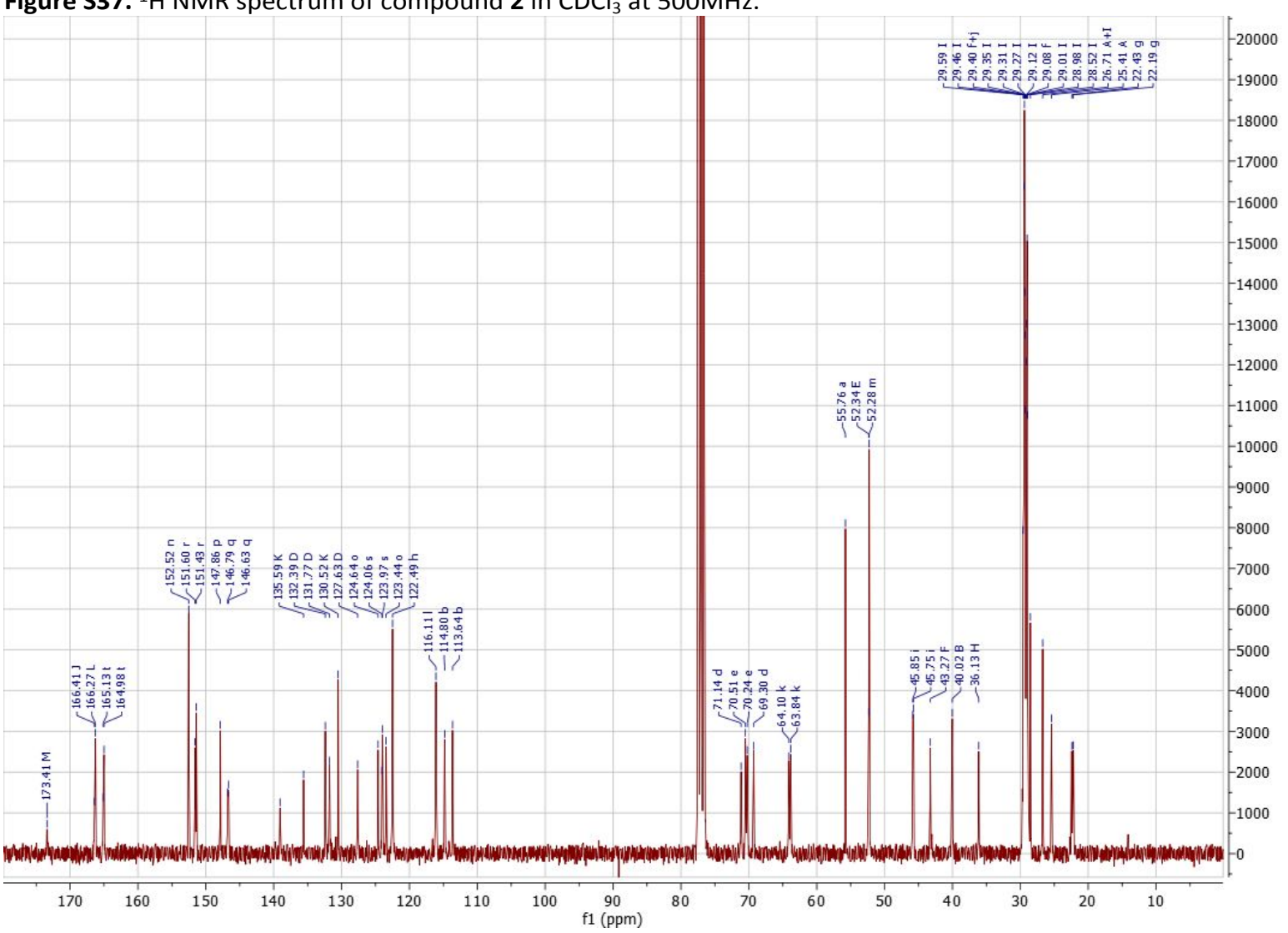

Figure S38. ${ }^{13} \mathrm{C}$ NMR spectrum of compound 2 in $\mathrm{CDCl}_{3}$ at $500 \mathrm{MHz}$. 


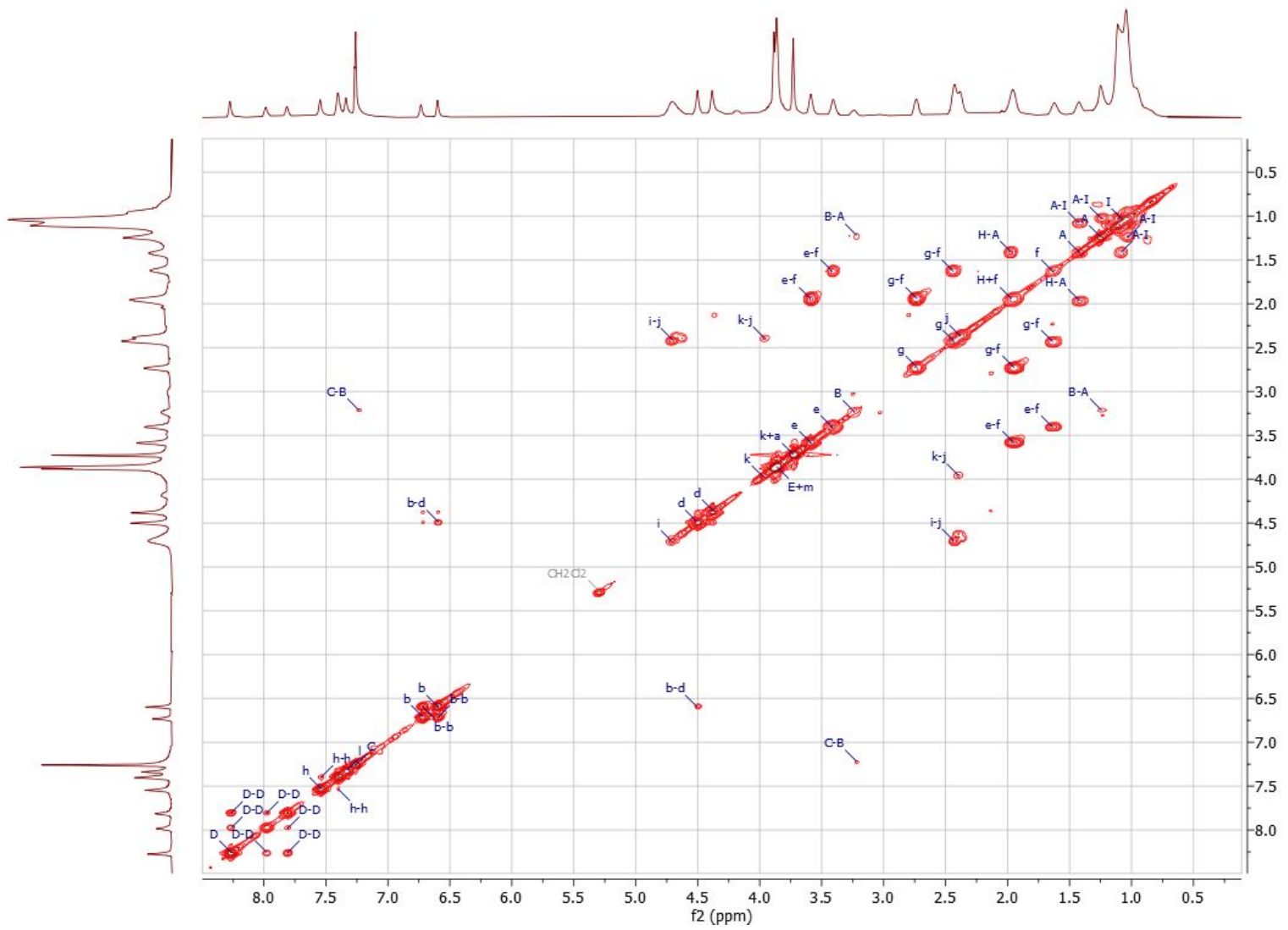

$\underset{\mathrm{E}}{\widehat{\mathrm{a}}}$

Figure S39. COSY NMR spectrum of compound 2 in $\mathrm{CDCl}_{3}$ at $500 \mathrm{MHz}$.

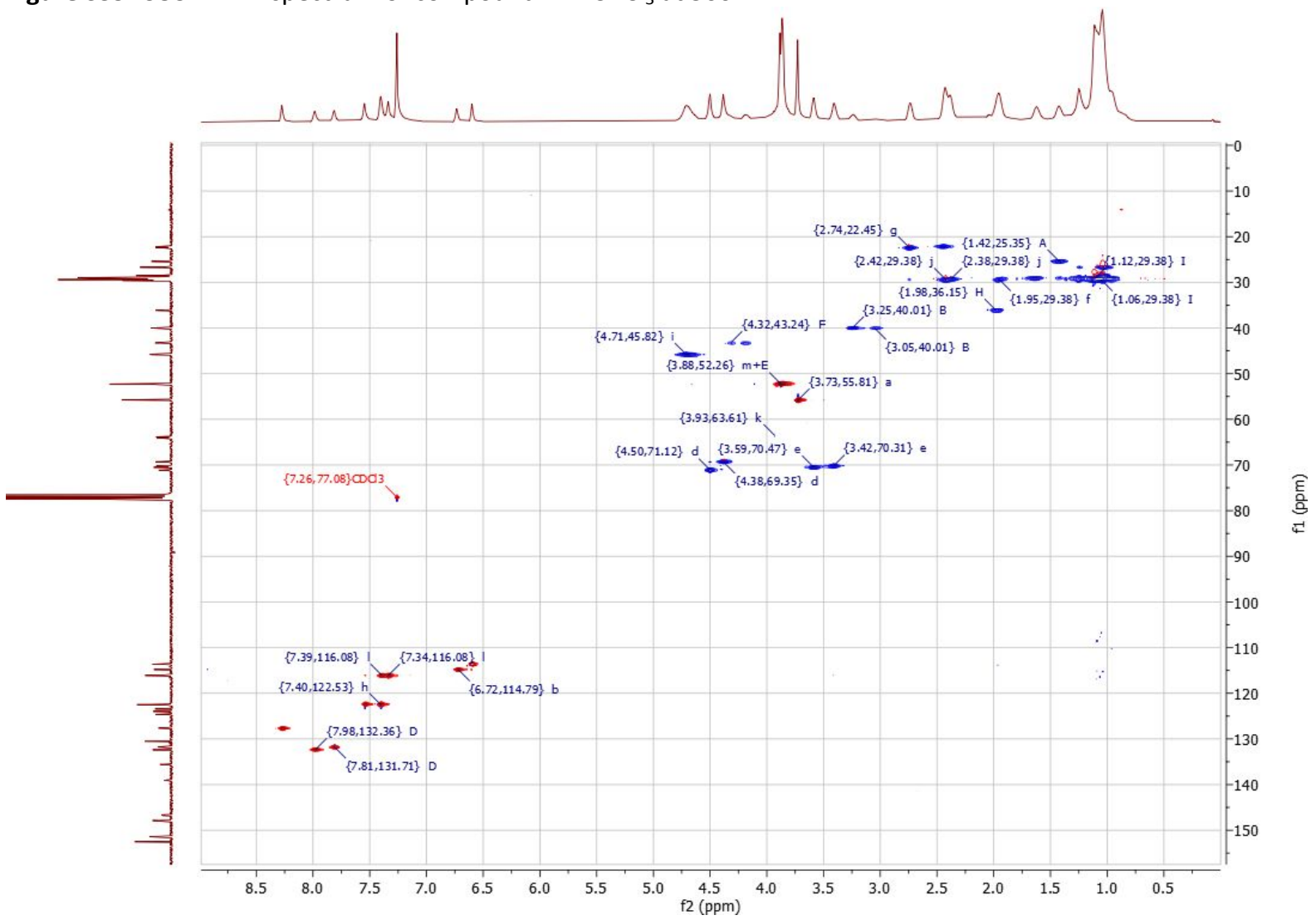

Figure S40. HSQC NMR spectrum of compound $\mathbf{2}$ in $\mathrm{CDCl}_{3}$ (overview) at $500 \mathrm{MHz}$. 


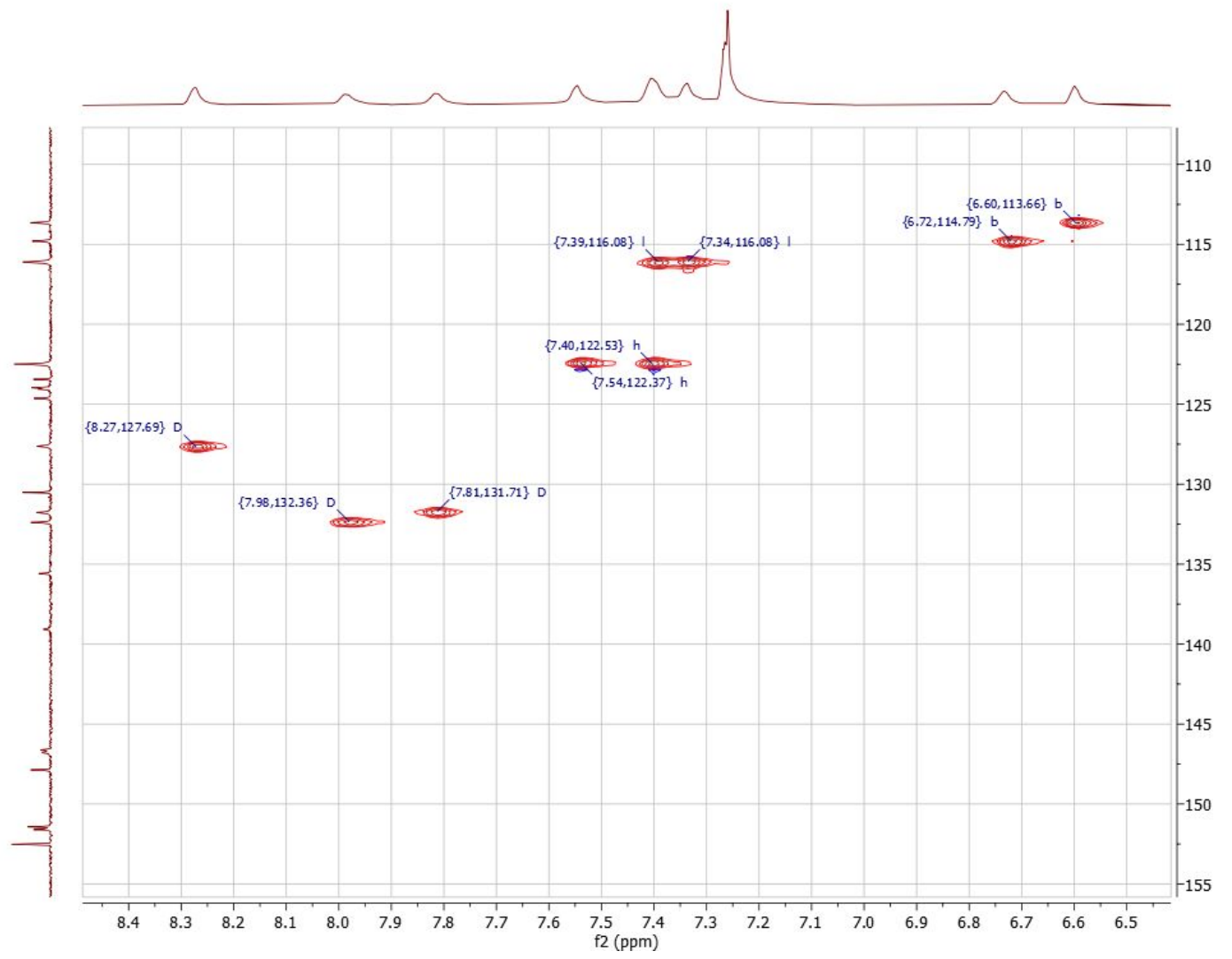

Figure S41. HSQC NMR spectrum of compound $\mathbf{2}$ in $\mathrm{CDCl}_{3}$ (aromatic region) at $500 \mathrm{MHz}$.

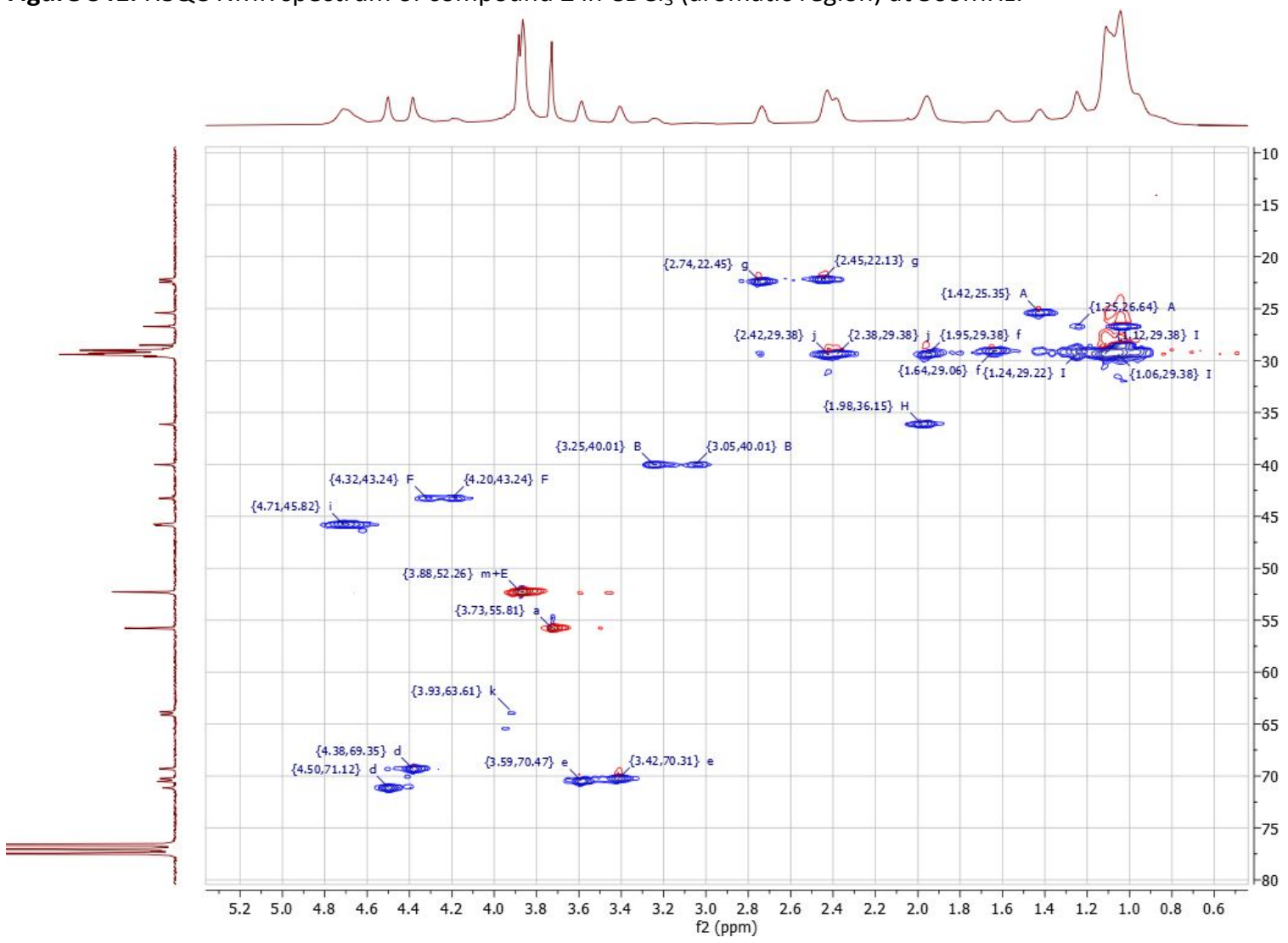

Figure S42. HSQC NMR spectrum of compound 2 in $\mathrm{CDCl}_{3}$ (aliphatic region) at $500 \mathrm{MHz}$. 

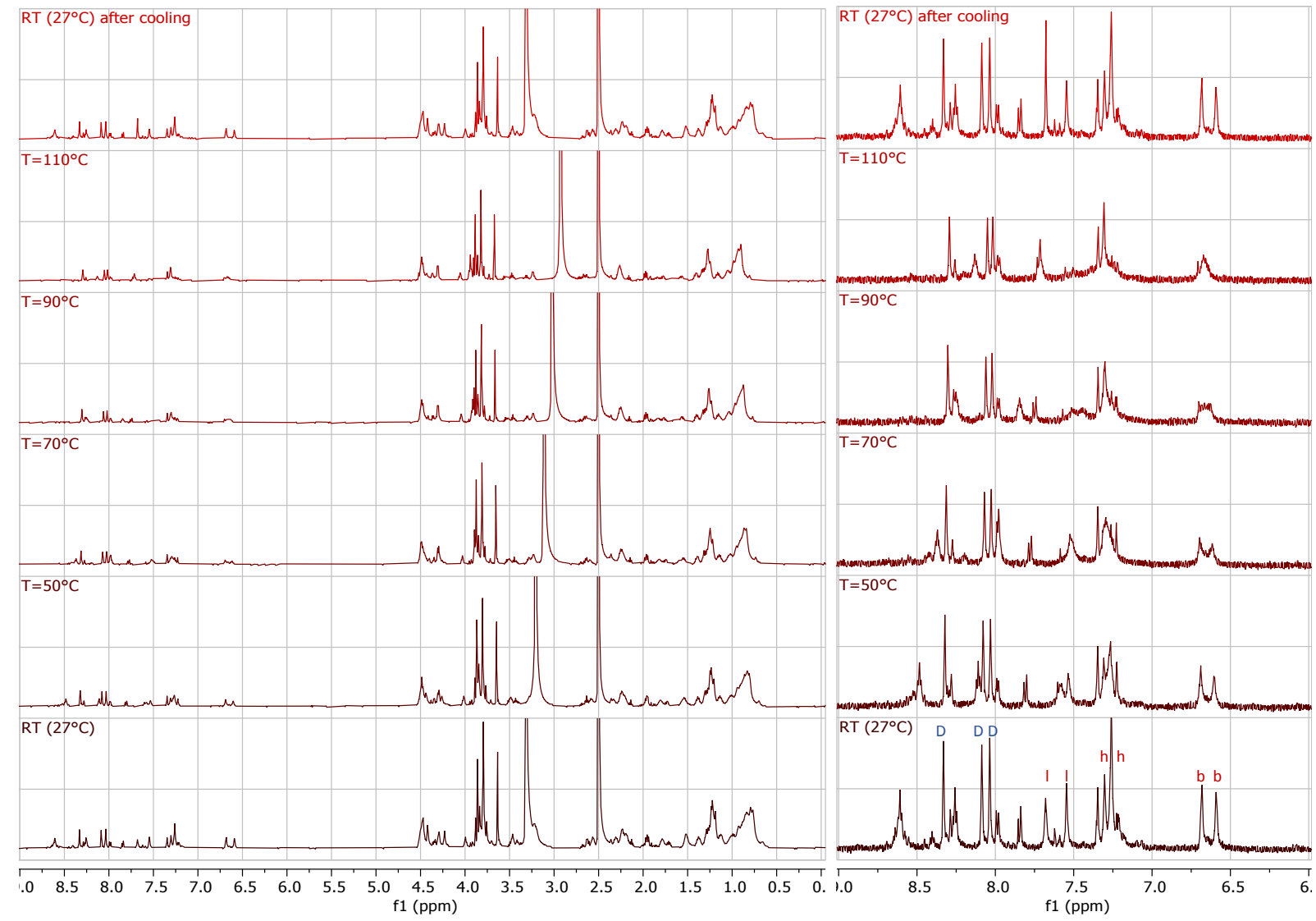

Figure S43. Variable temperature NMR spectra of compound $\mathbf{2}$ in $\left(\mathrm{CD}_{3}\right)_{2} \mathrm{SO}$ at $500 \mathrm{MHz}$. 


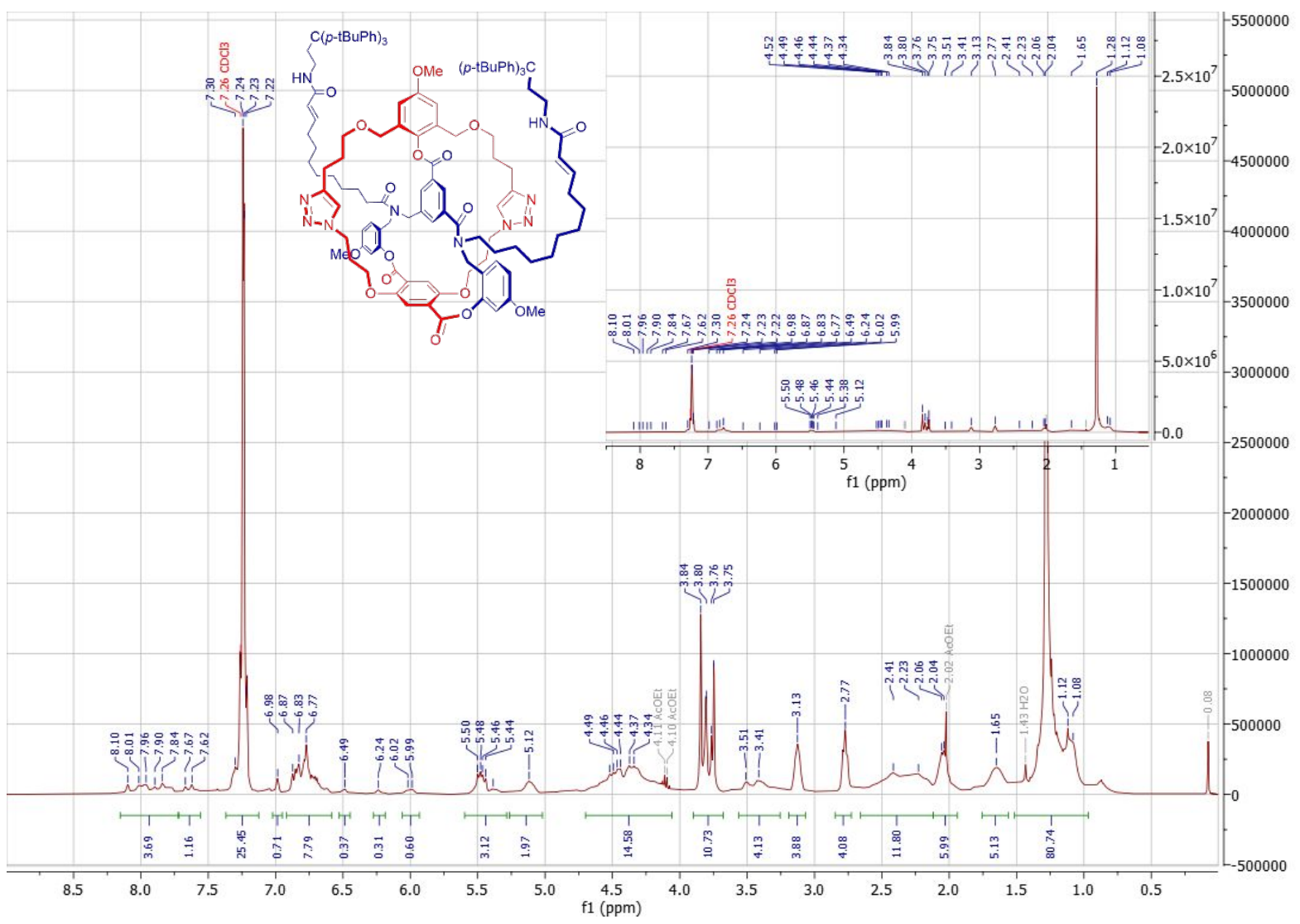

Figure S44. ${ }^{1} \mathrm{H}$ NMR spectrum of compound 12 in $\mathrm{CDCl}_{3}$ at $400 \mathrm{MHz}$.

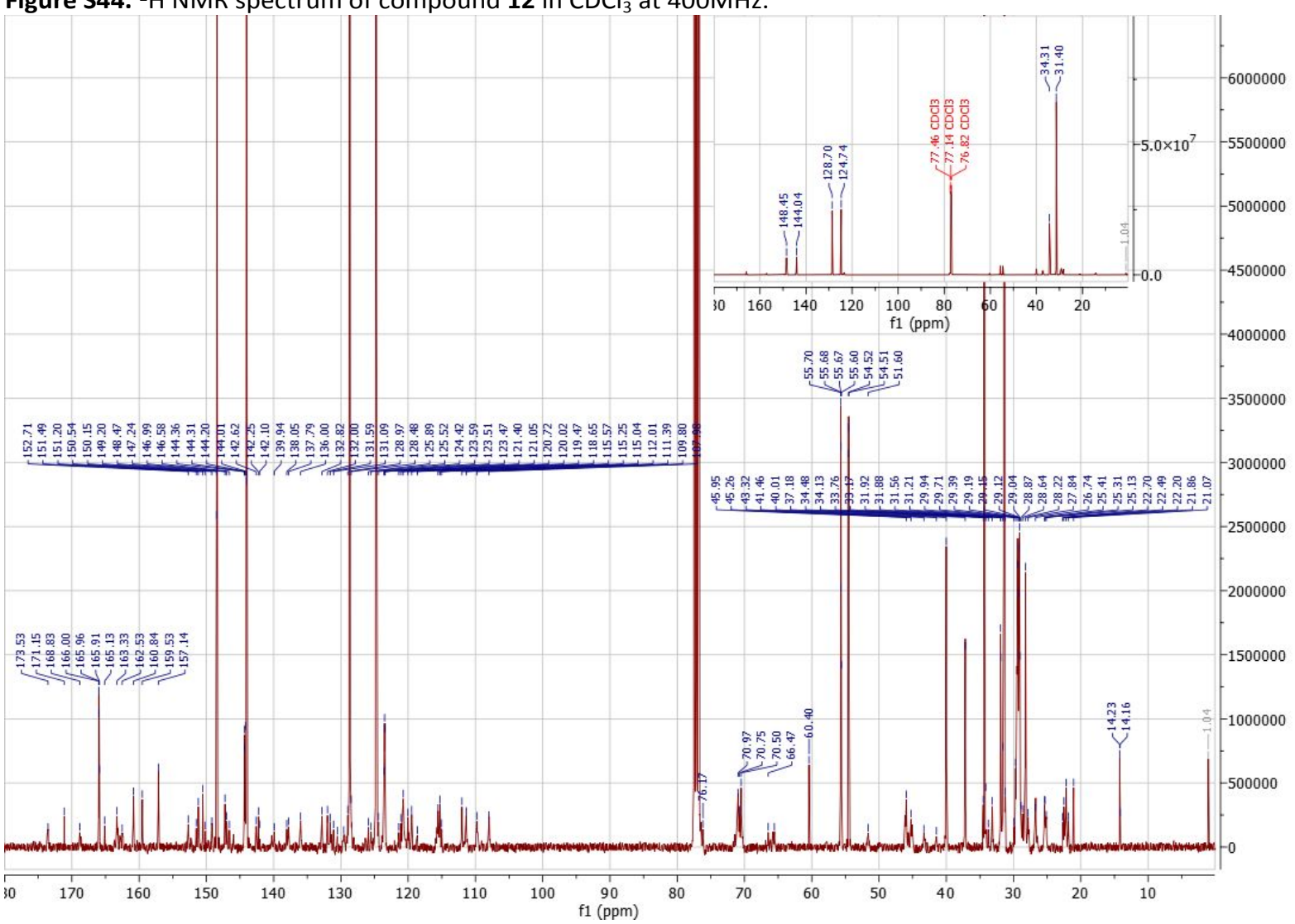

Figure S45. ${ }^{13} \mathrm{C}$ NMR spectrum of compound 12 in $\mathrm{CDCl}_{3}$ at $400 \mathrm{MHz}$. 


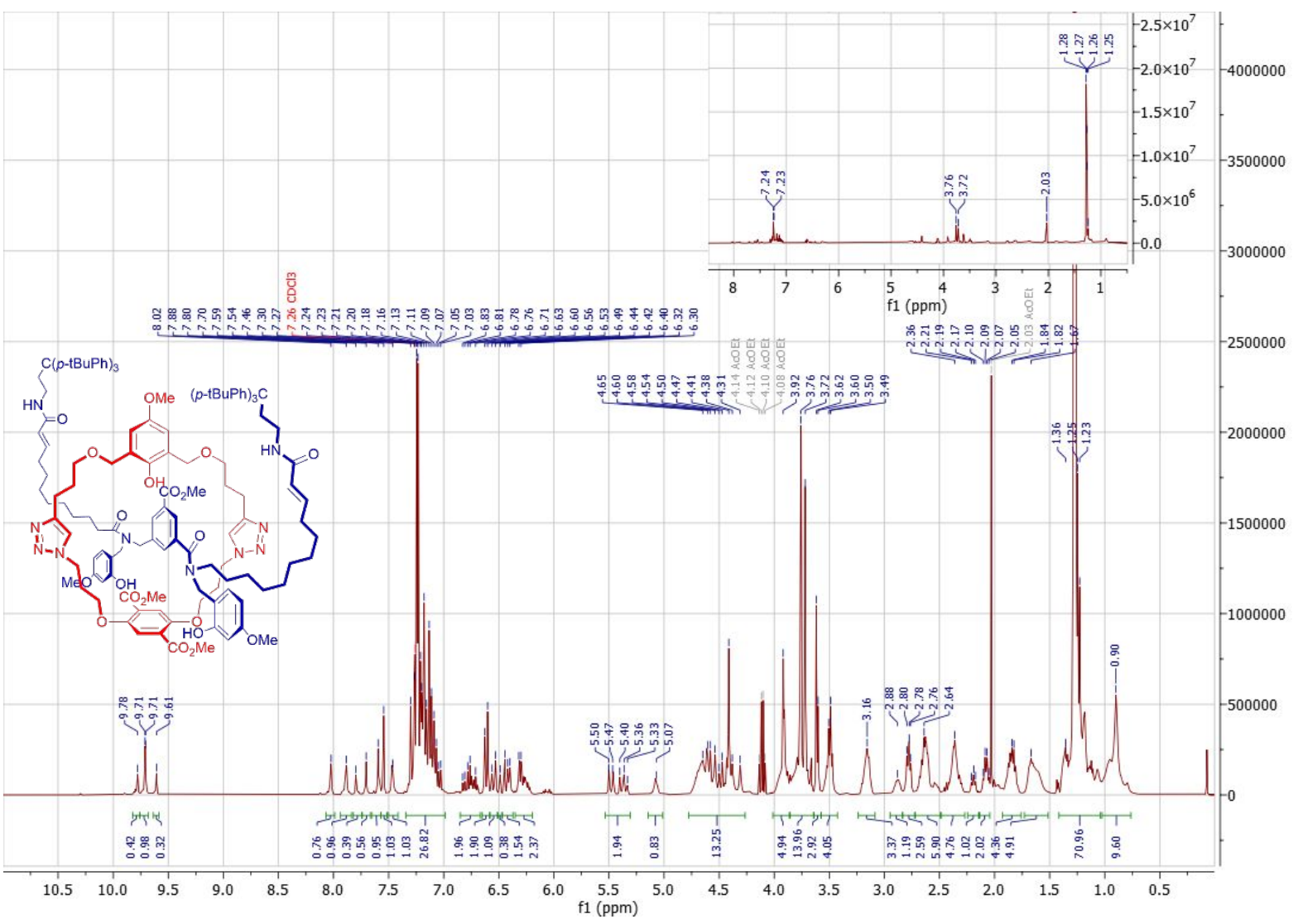

Figure S46. ${ }^{1} \mathrm{H}$ NMR spectrum of compound 13 in $\mathrm{CDCl}_{3}$ at $400 \mathrm{MHz}$.

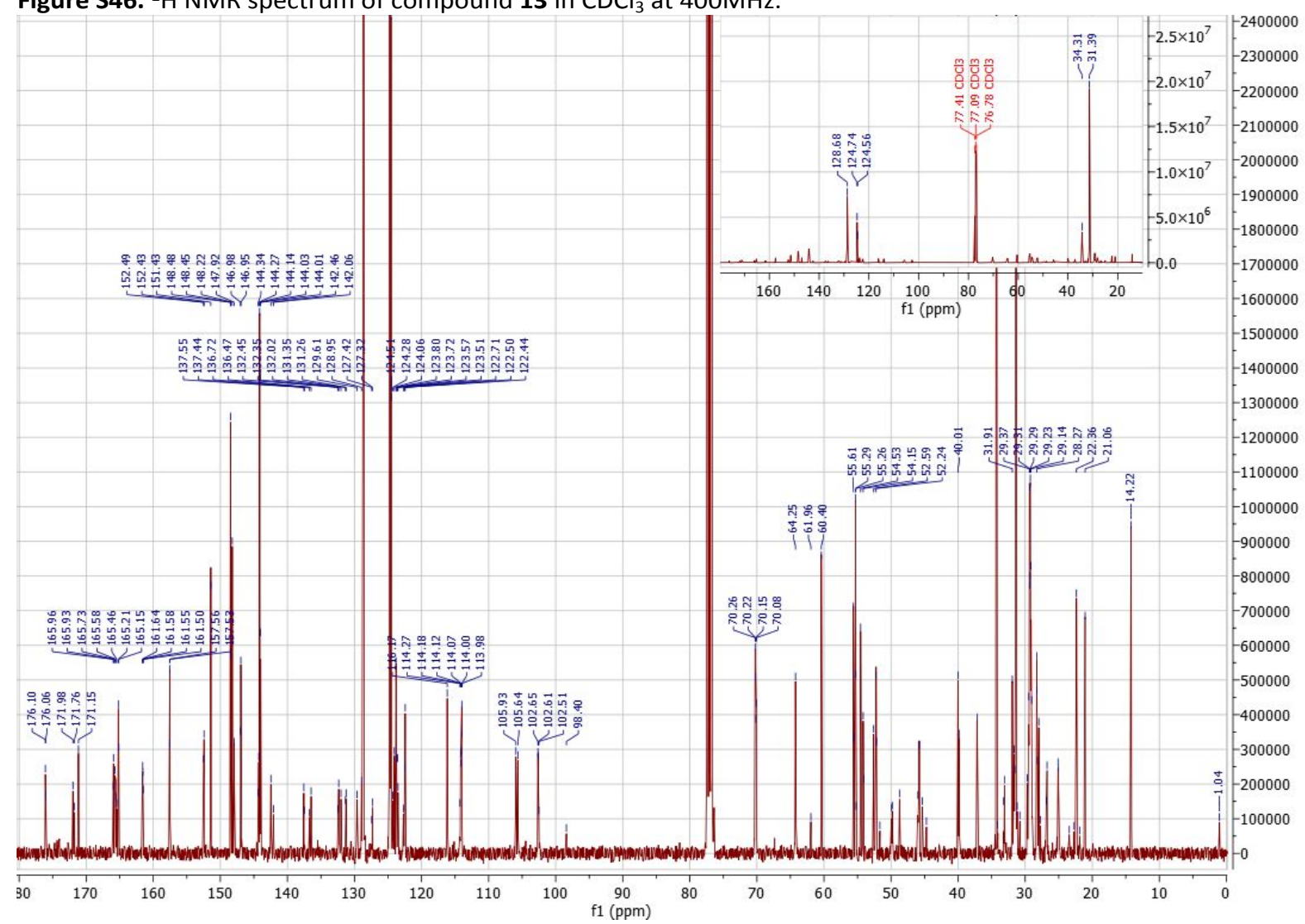

Figure S47. ${ }^{13} \mathrm{C}$ NMR spectrum of compound 13 in $\mathrm{CDCl}_{3}$ at $400 \mathrm{MHz}$. 


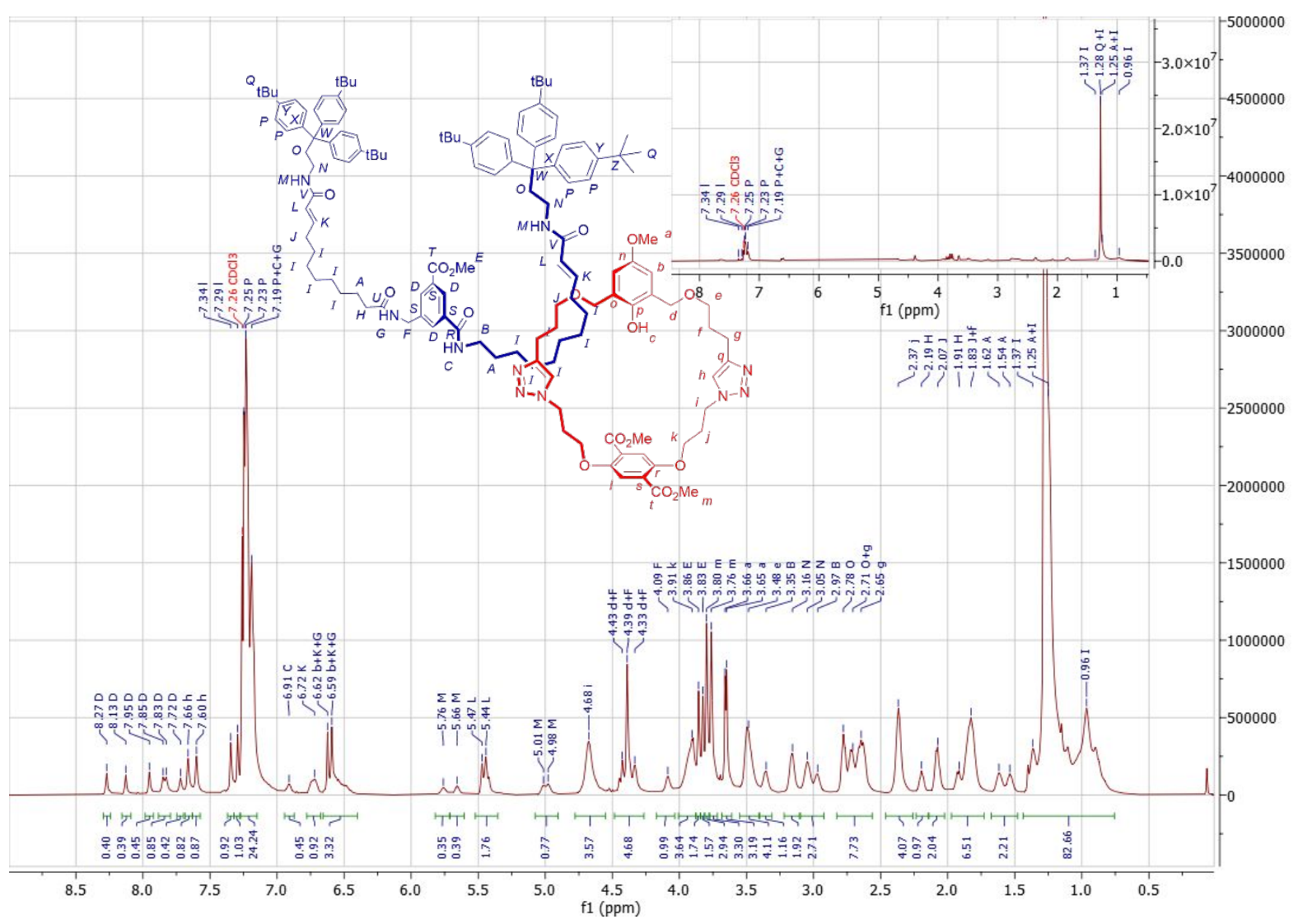

Figure S48. ${ }^{1} \mathrm{H}$ NMR spectrum of compound 3 in $\mathrm{CDCl}_{3}$ at $500 \mathrm{MHz}$.

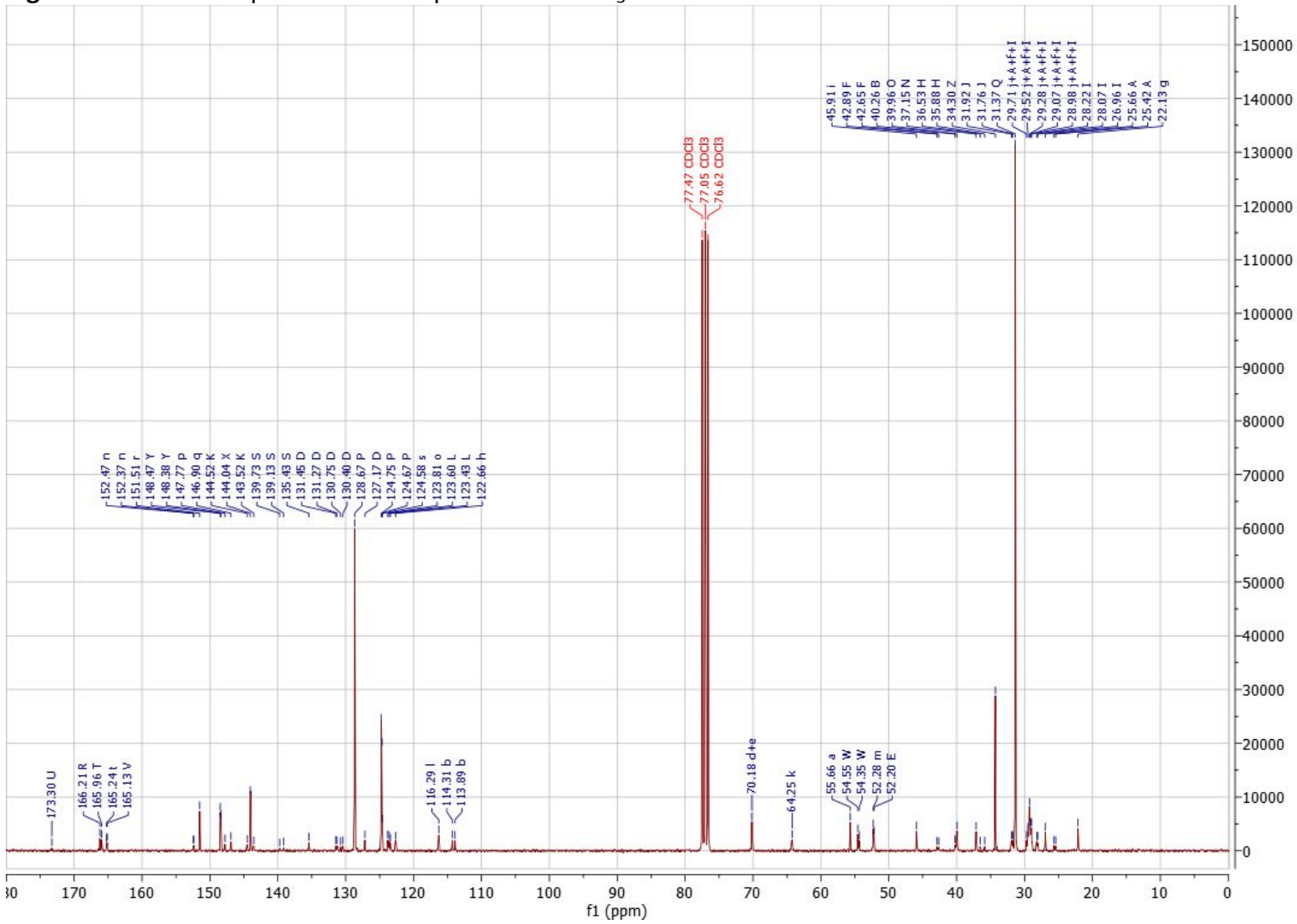

Figure S49. ${ }^{13} \mathrm{C}$ NMR spectrum of compound 3 in $\mathrm{CDCl}_{3}$ (overview) at $500 \mathrm{MHz}$. 


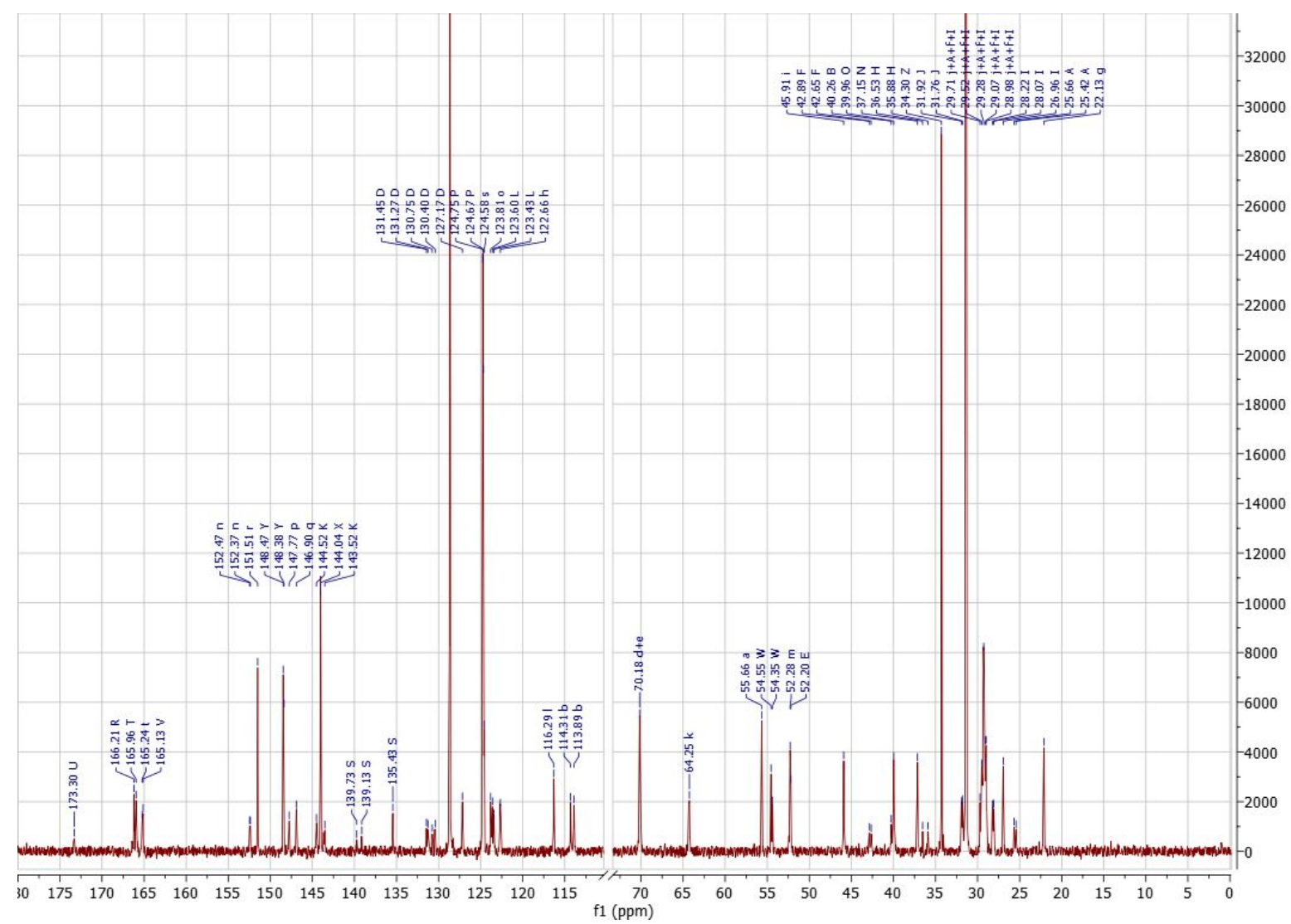

Figure S50. ${ }^{13} \mathrm{C}$ NMR spectrum of compound 3 in $\mathrm{CDCl}_{3}$ (detail) at $500 \mathrm{MHz}$.

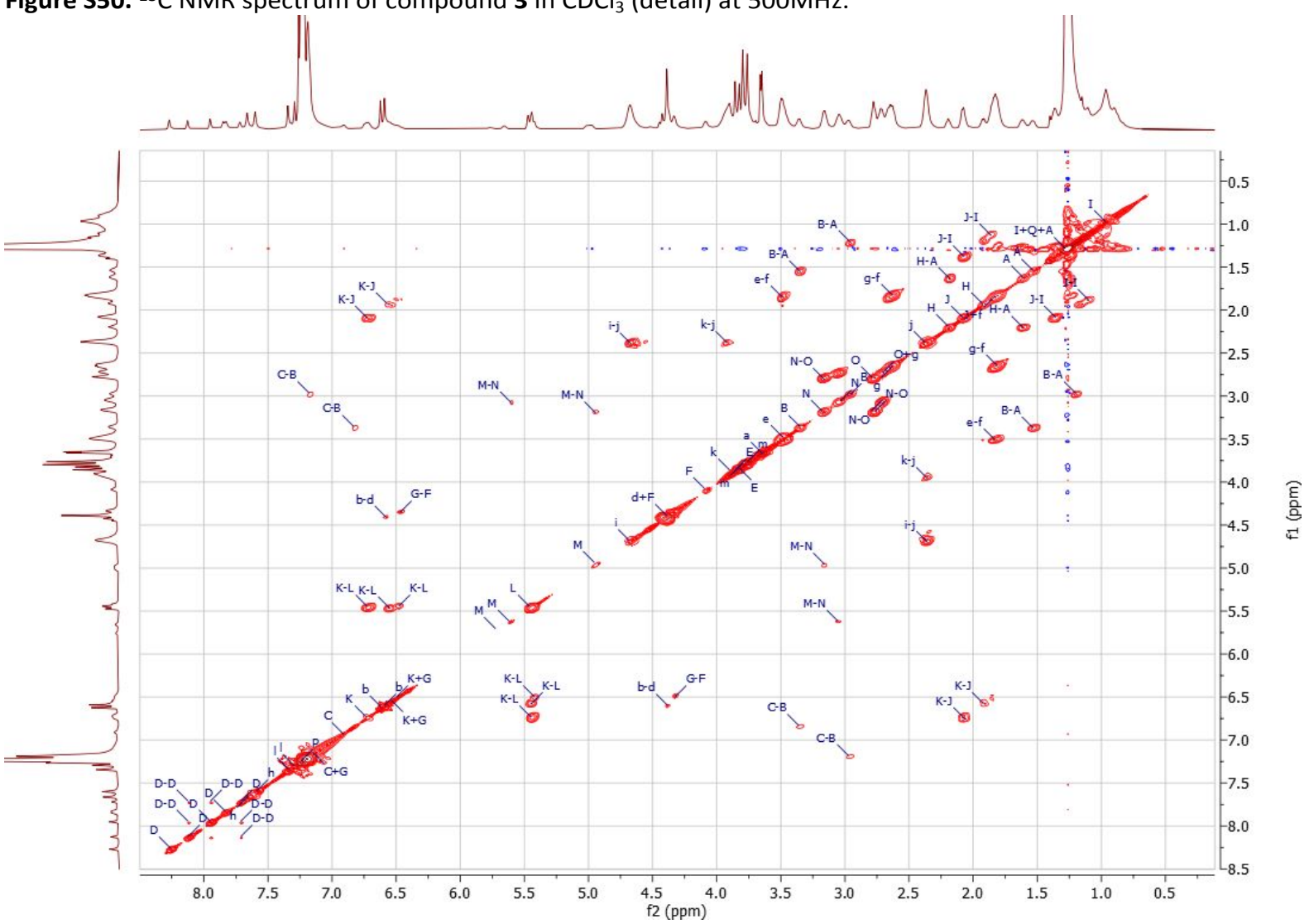

Figure S51. COSY NMR spectrum of compound 3 in $\mathrm{CDCl}_{3}$ (overview) at $500 \mathrm{MHz}$. 


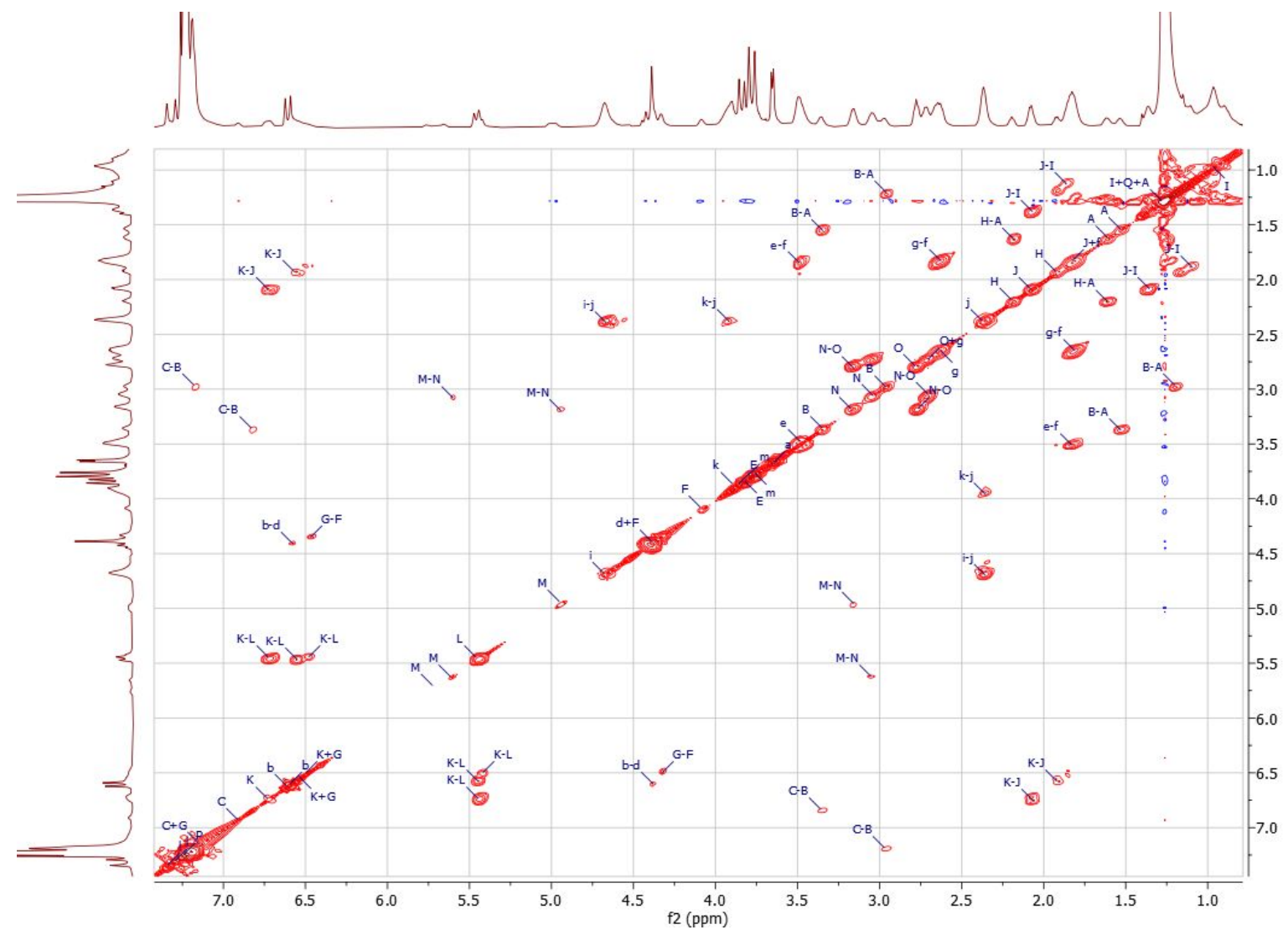

$\underset{\substack{0 \\ \text { a }}}{\overparen{1}}$

Figure S52. COSY NMR spectrum of compound $\mathbf{3}$ in $\mathrm{CDCl}_{3}$ (detail) at $500 \mathrm{MHz}$.

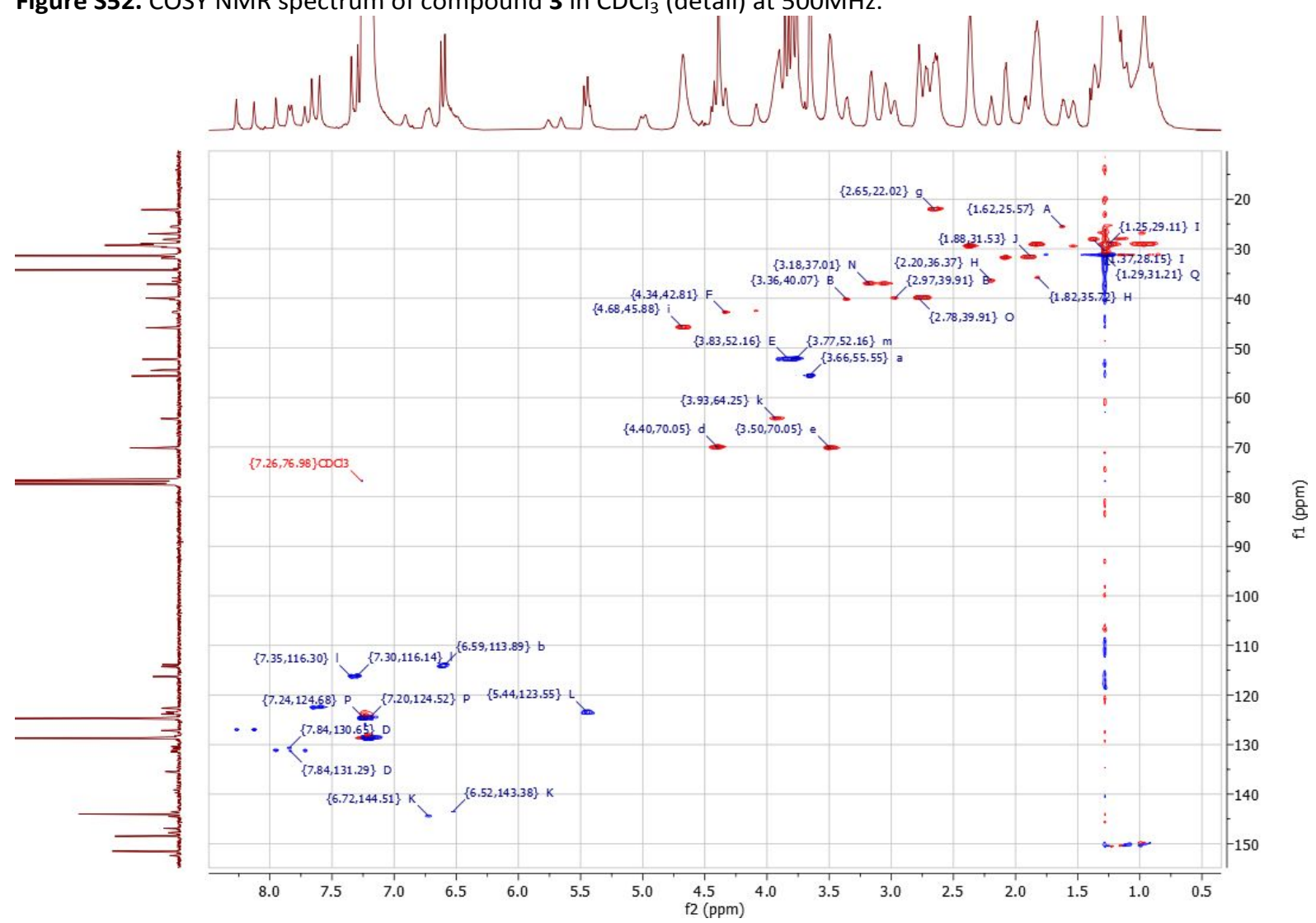

Figure S53. HSQC NMR spectrum of compound 3 in $\mathrm{CDCl}_{3}$ (overview) at $500 \mathrm{MHz}$. 


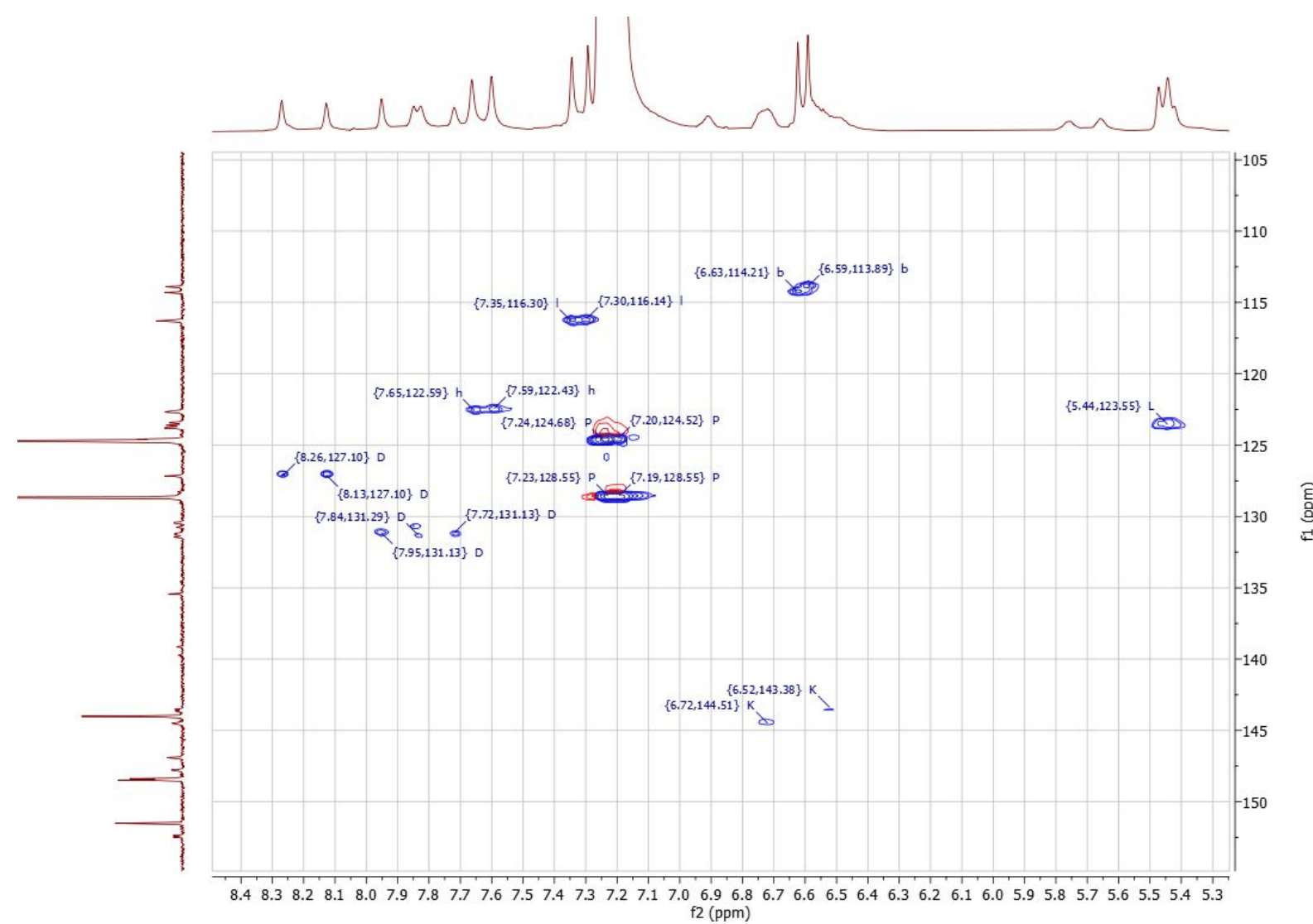

Figure S54. HSQC NMR spectrum of compound 3 in $\mathrm{CDCl}_{3}$ (aromatic region) at $500 \mathrm{MHz}$.

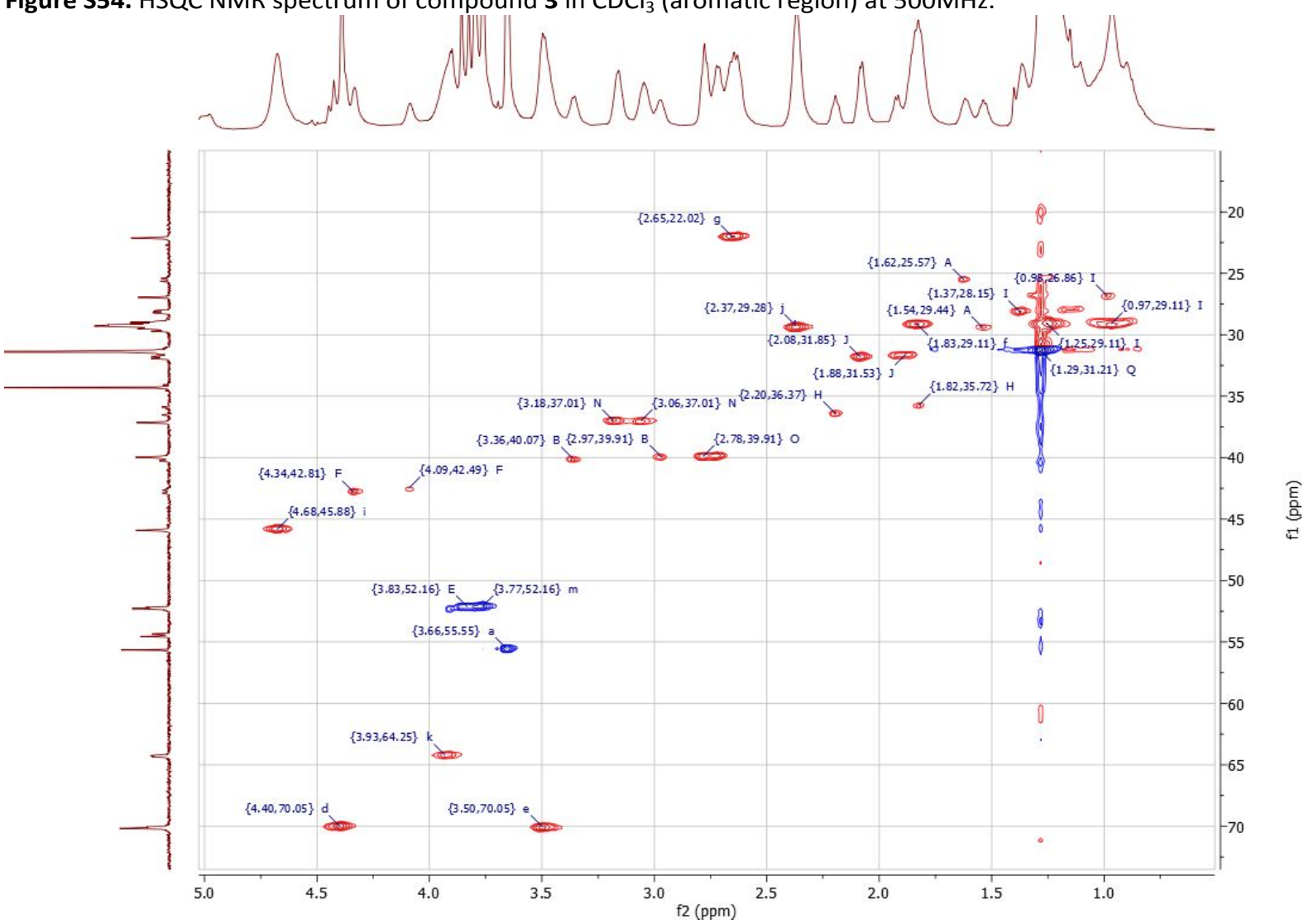

Figure S55. HSQC NMR spectrum of compound 3 in $\mathrm{CDCl}_{3}$ (aliphatic region) at $500 \mathrm{MHz}$. 


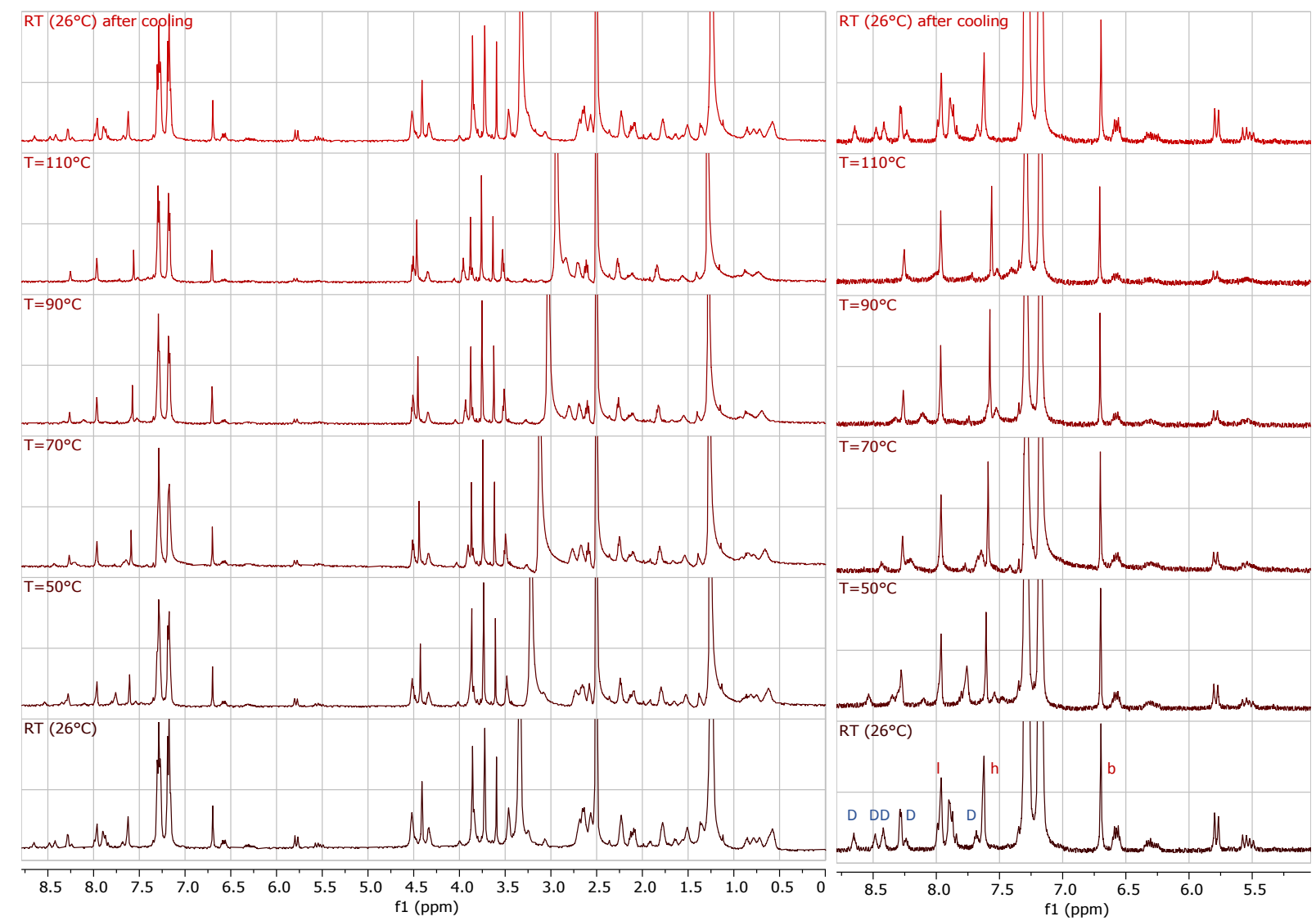

Figure S56. Variable temperature NMR spectra of compound $\mathbf{3}$ in $\left(\mathrm{CD}_{3}\right)_{2} \mathrm{SO}$ at $500 \mathrm{MHz}$. 


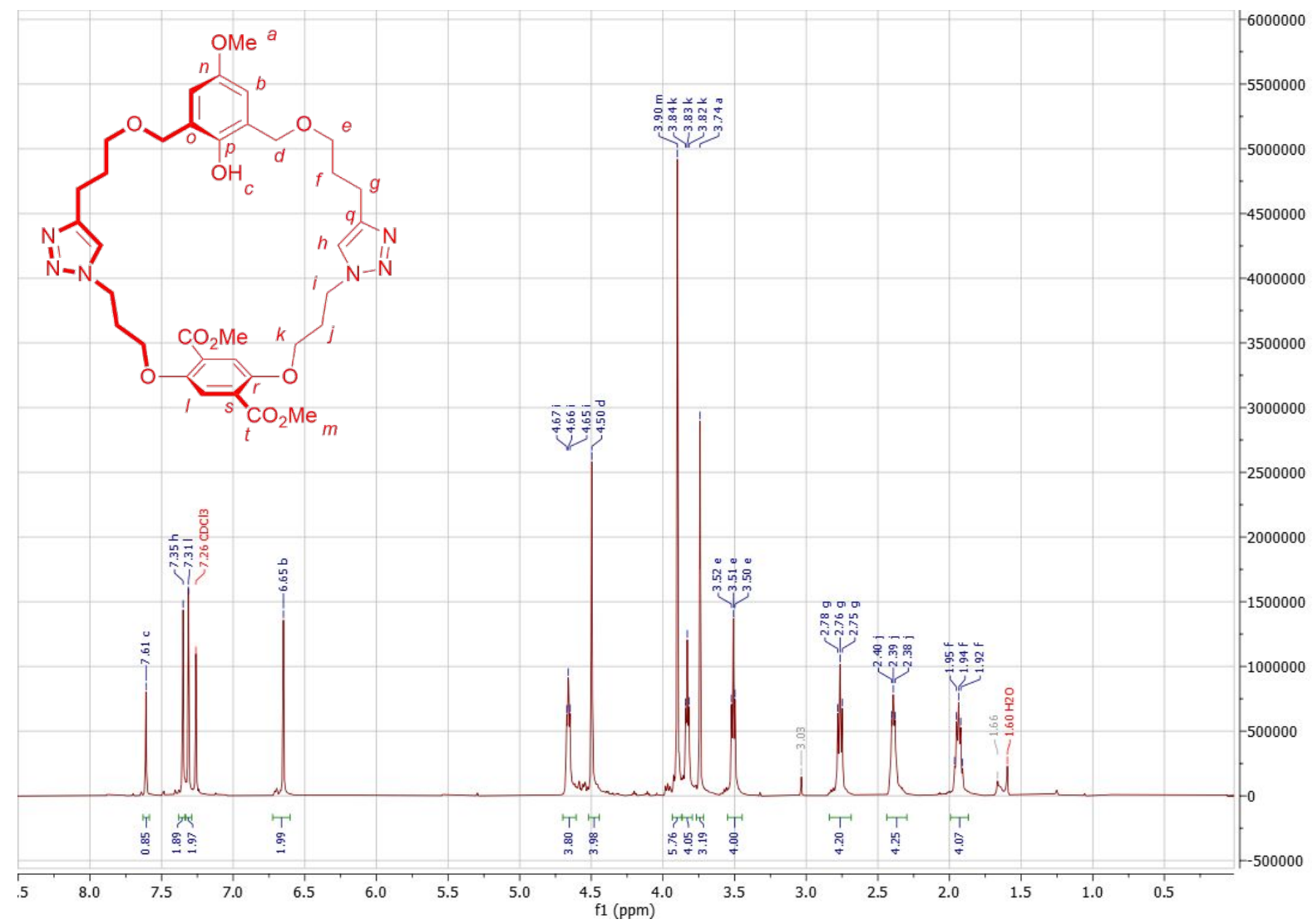

Figure S57. ${ }^{1} \mathrm{H}$ NMR spectrum of compound 26 in $\mathrm{CDCl}_{3}$ at $500 \mathrm{MHz}$.

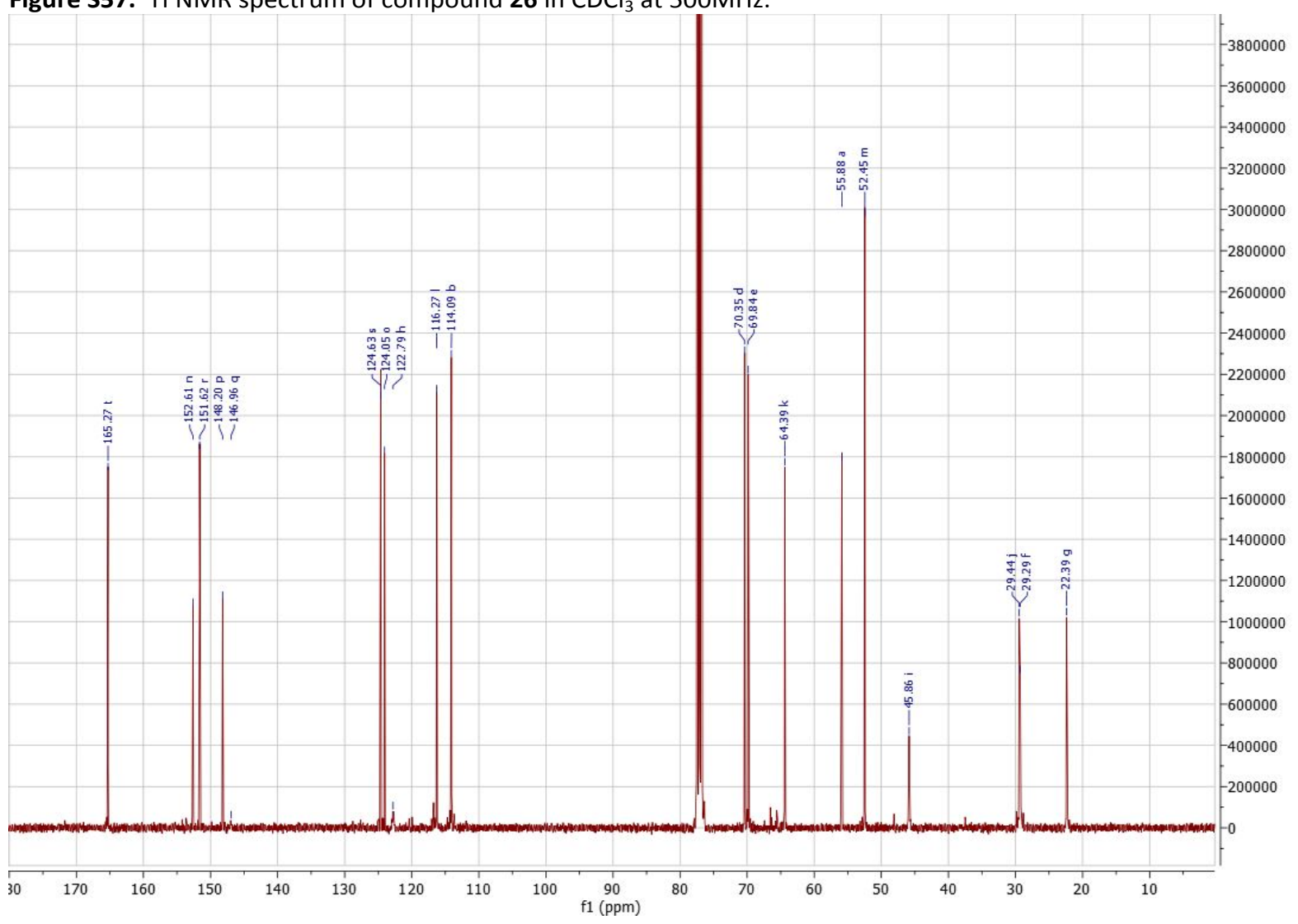

Figure S58. ${ }^{13} \mathrm{C} \mathrm{NMR} \mathrm{spectrum} \mathrm{of} \mathrm{compound} 26$ in $\mathrm{CDCl}_{3}$ at $500 \mathrm{MHz}$. Note triazole signals $q$ and $h$ are barely visible at room temperature, a second ${ }^{13} \mathrm{C}$ NMR spectrum at $-20^{\circ} \mathrm{C}$ is available were these peaks appear sharper. 


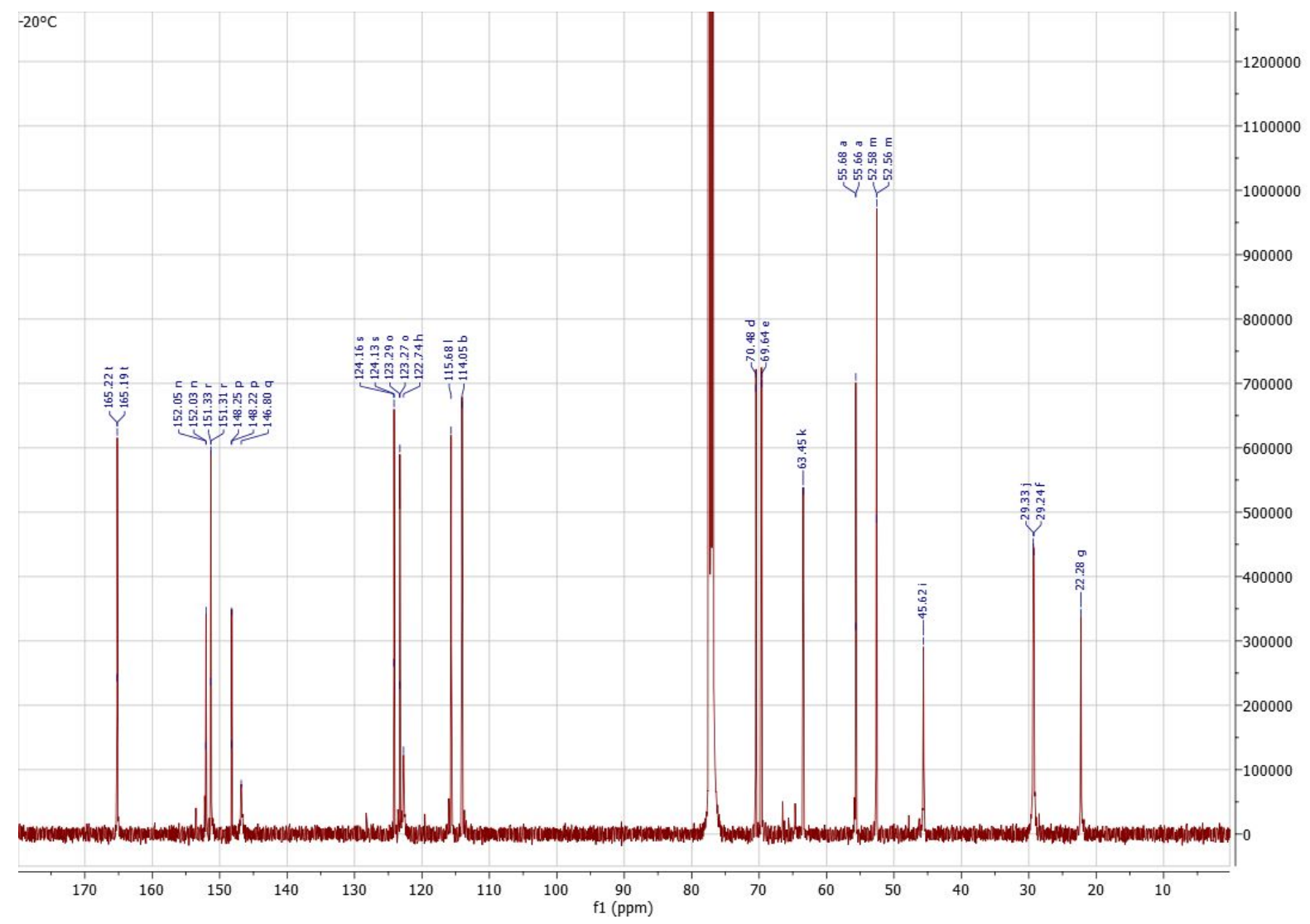

Figure S59. ${ }^{13} \mathrm{C} \mathrm{NMR} \mathrm{spectrum} \mathrm{of} \mathrm{compound} 26$ in $\mathrm{CDCl}_{3}$ at $-20^{\circ} \mathrm{C}$ at $500 \mathrm{MHz}$.

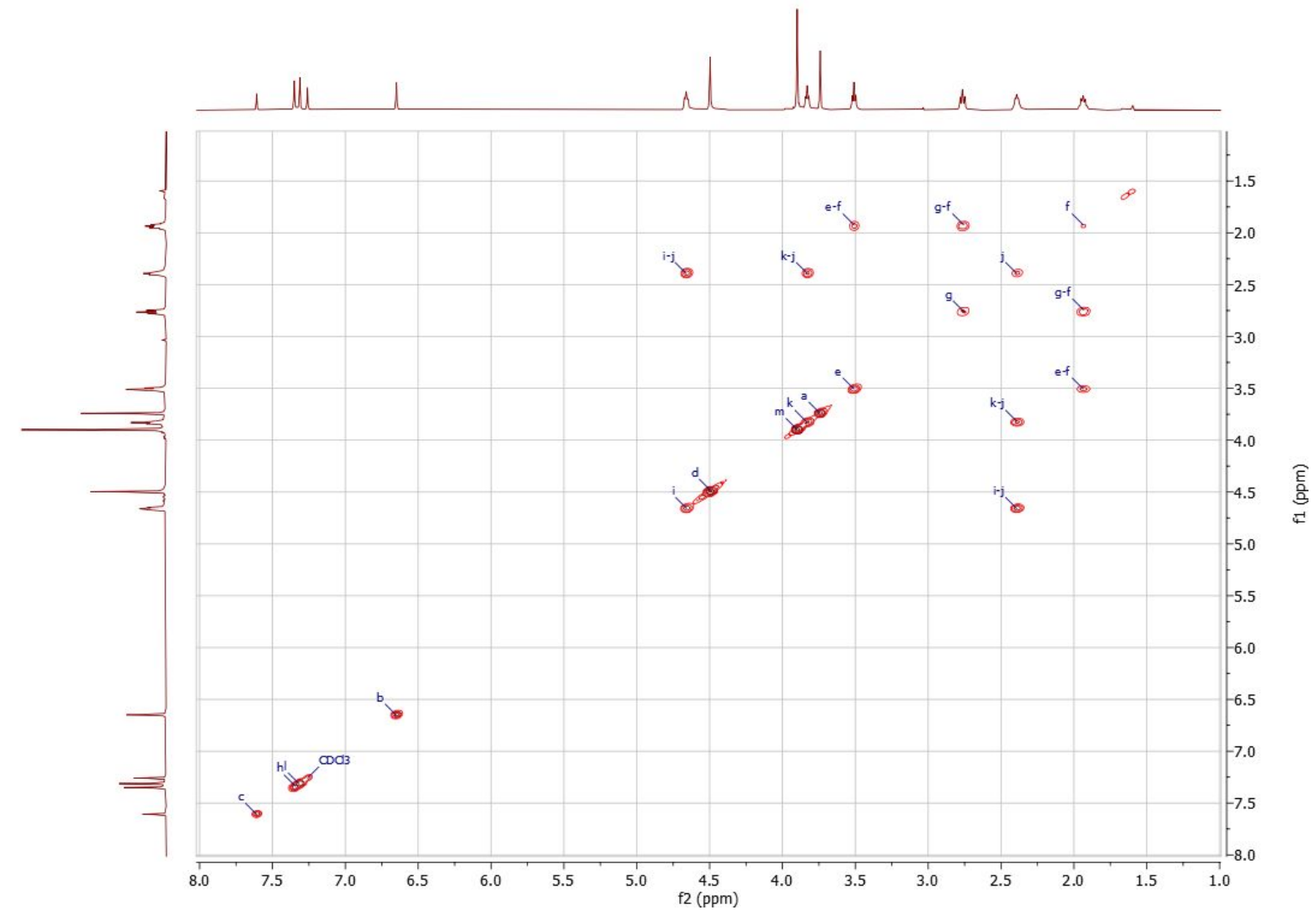

Figure S60. COSY NMR spectrum of compound 26 in $\mathrm{CDCl}_{3}$ at $500 \mathrm{MHz}$. 


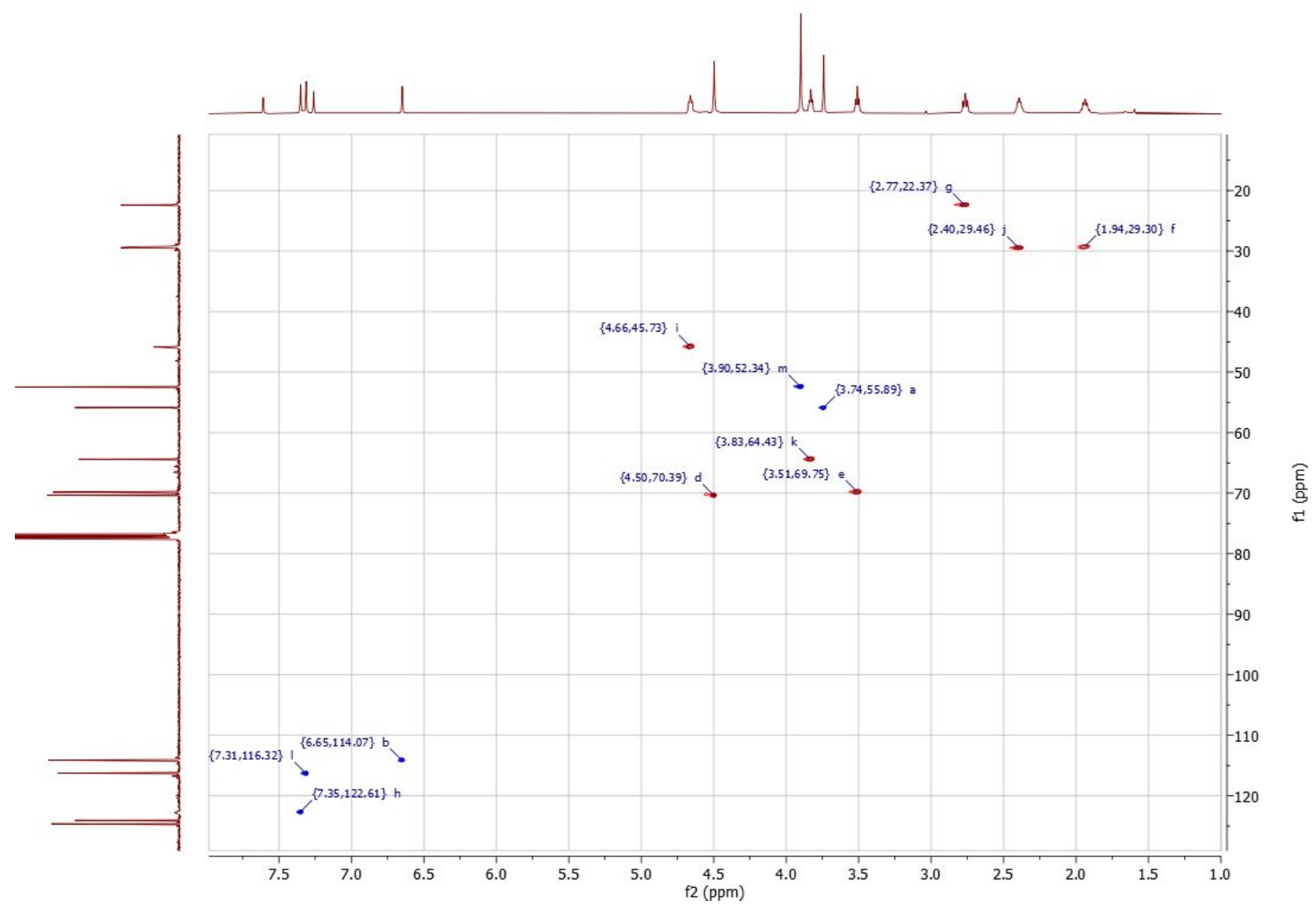

Figure S61. HSQC NMR spectrum of compound 26 in $\mathrm{CDCl}_{3}$ at $500 \mathrm{MHz}$. 


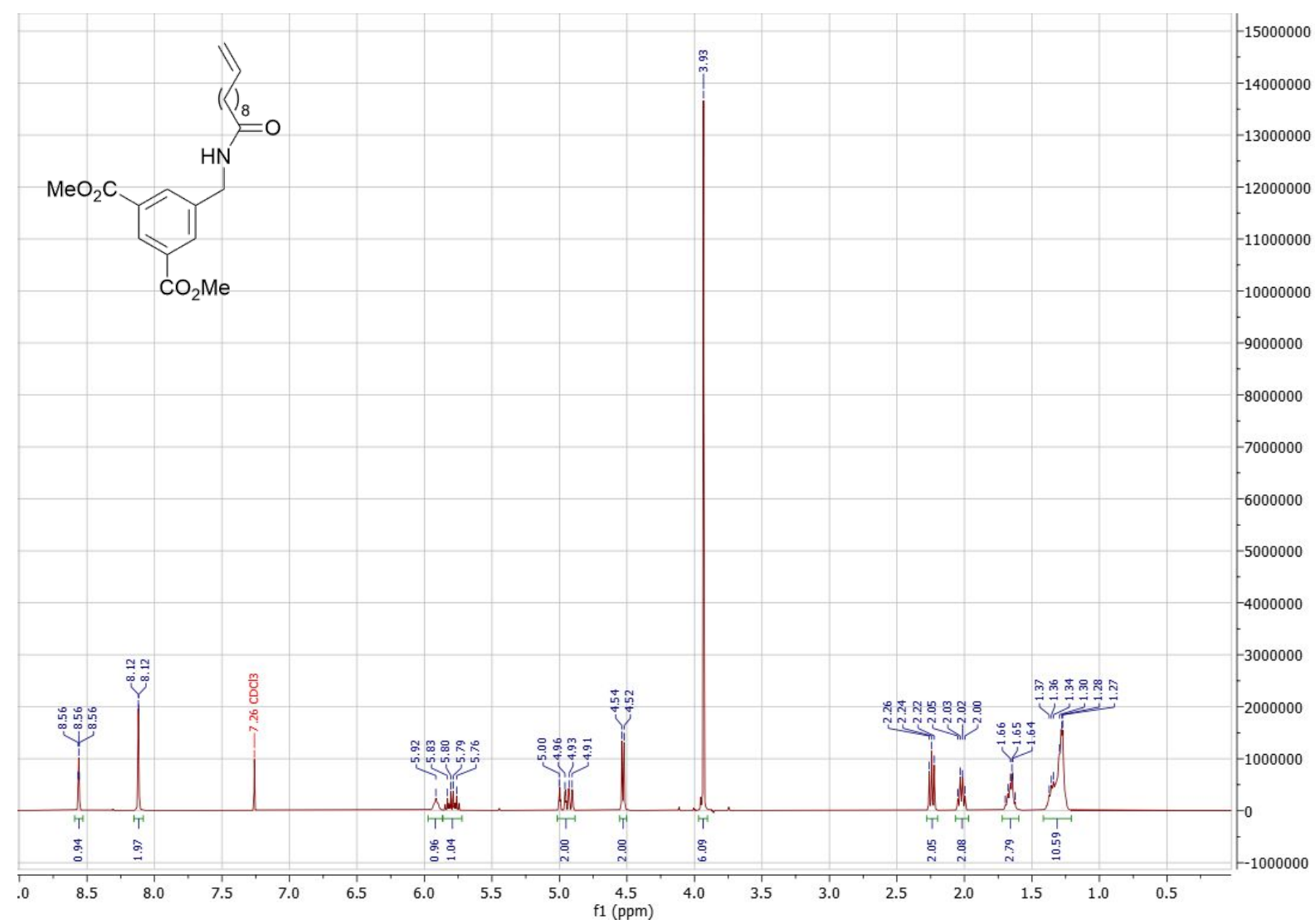

Figure S62. ${ }^{1} \mathrm{H}$ NMR spectrum of compound 27 in $\mathrm{CDCl}_{3}$ at $400 \mathrm{MHz}$.

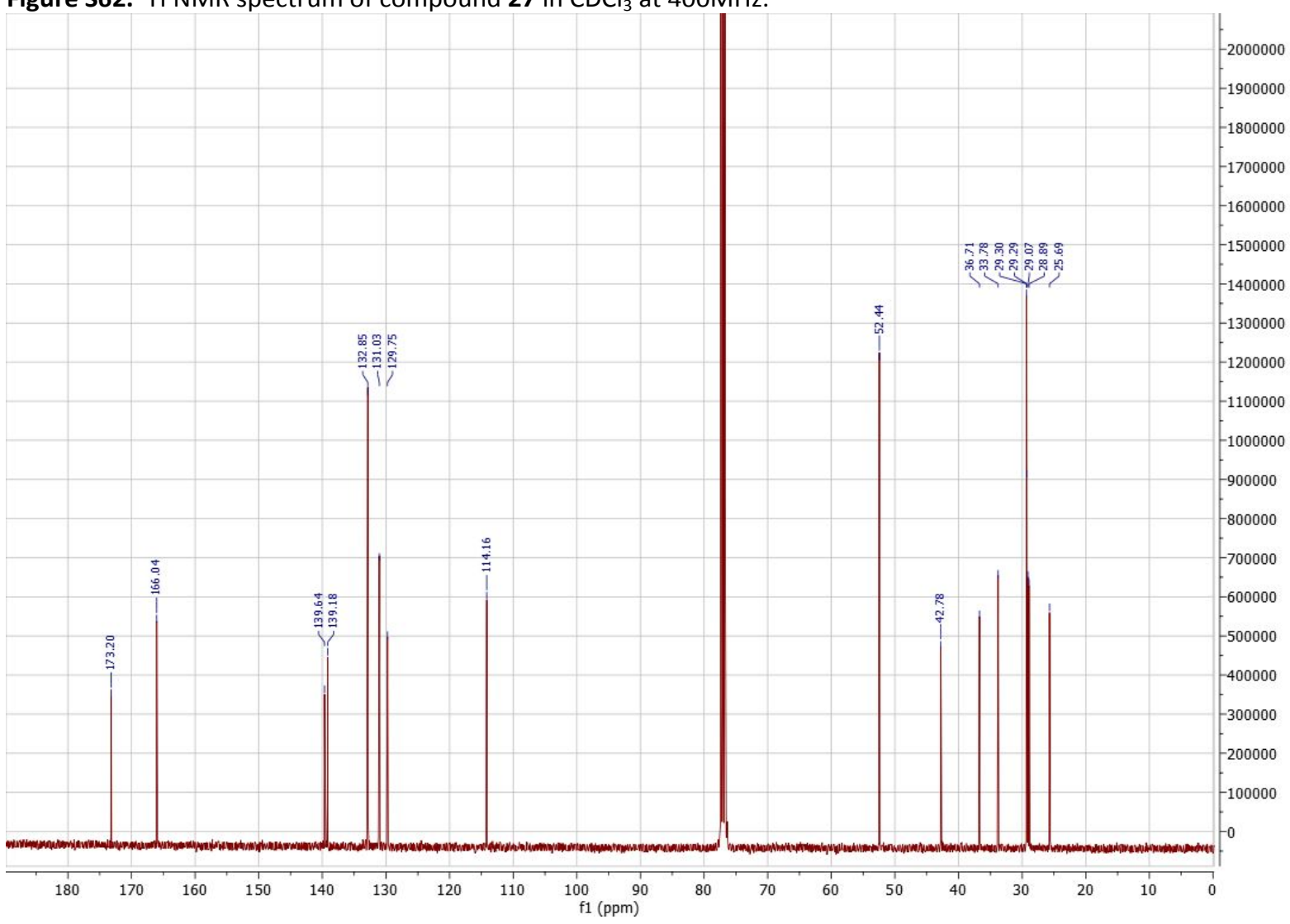

Figure S63. ${ }^{13} \mathrm{C}$ NMR spectrum of compound 27 in $\mathrm{CDCl}_{3}$ at $400 \mathrm{MHz}$. 


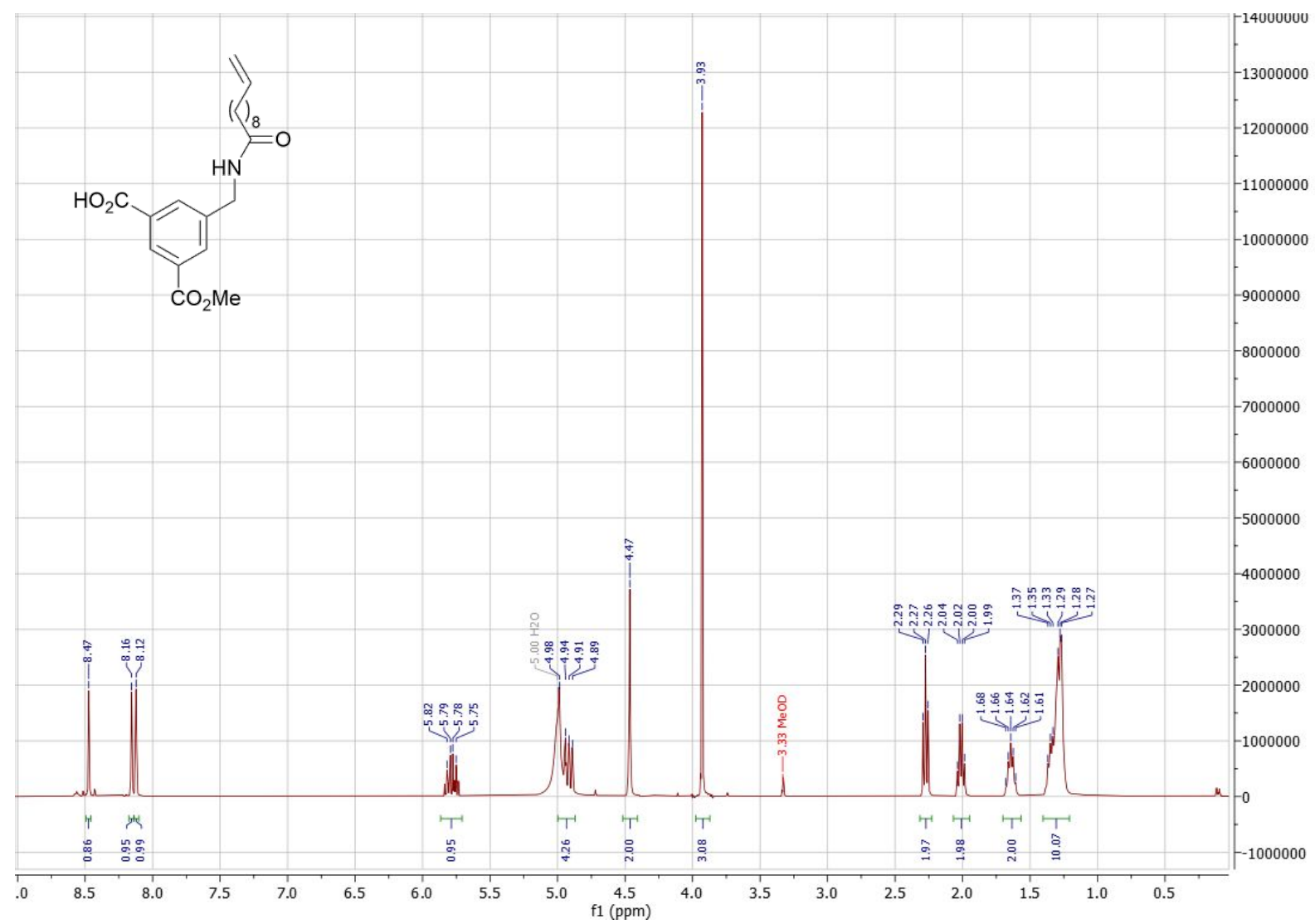

Figure S64. ${ }^{1} \mathrm{H}$ NMR spectrum of compound 28 in $\mathrm{CD}_{3} \mathrm{OD}$ at $400 \mathrm{MHz}$.

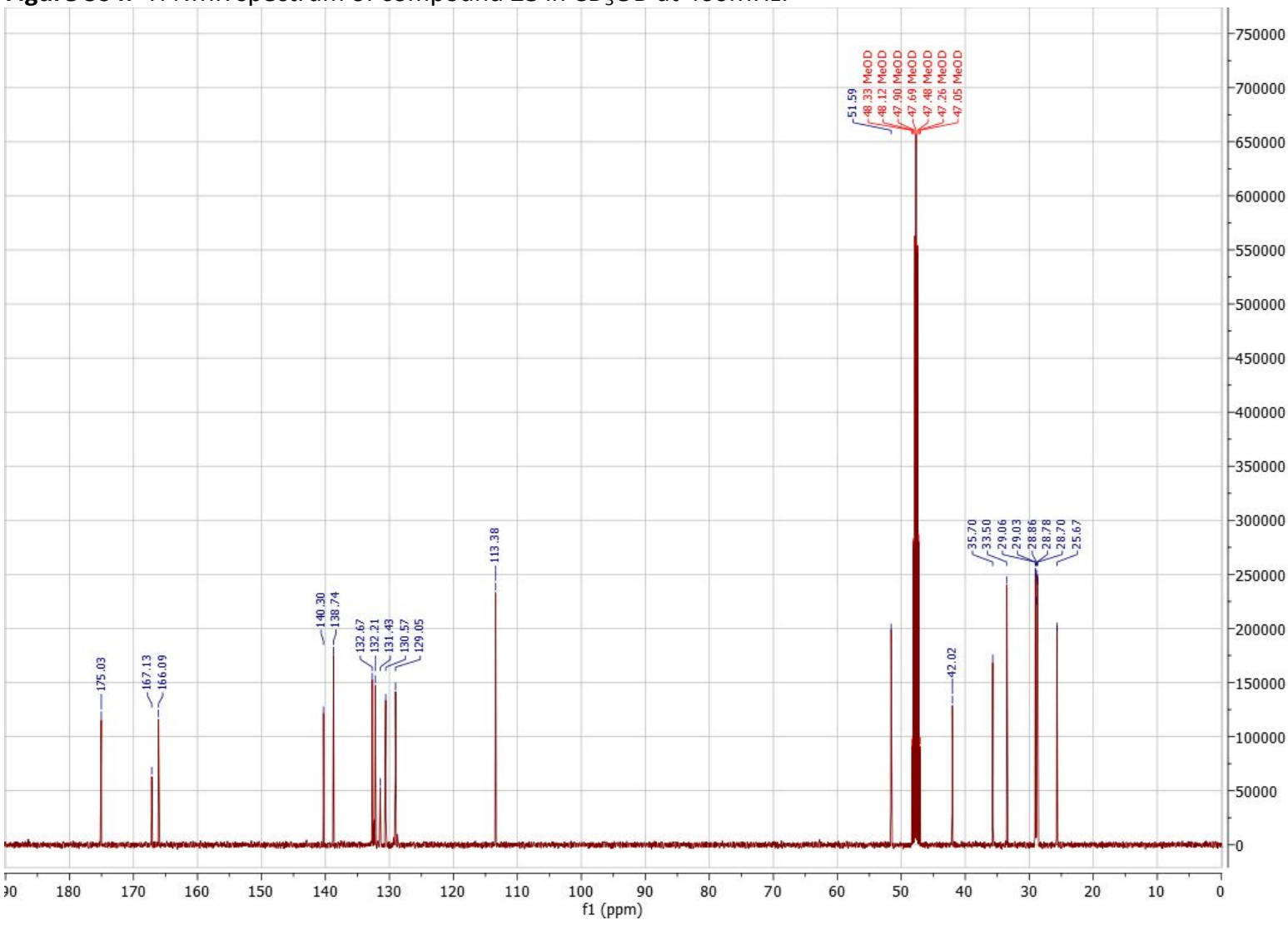

Figure S65. ${ }^{13} \mathrm{C}$ NMR spectrum of compound 28 in $\mathrm{CD}_{3} \mathrm{OD}$ at $400 \mathrm{MHz}$. 


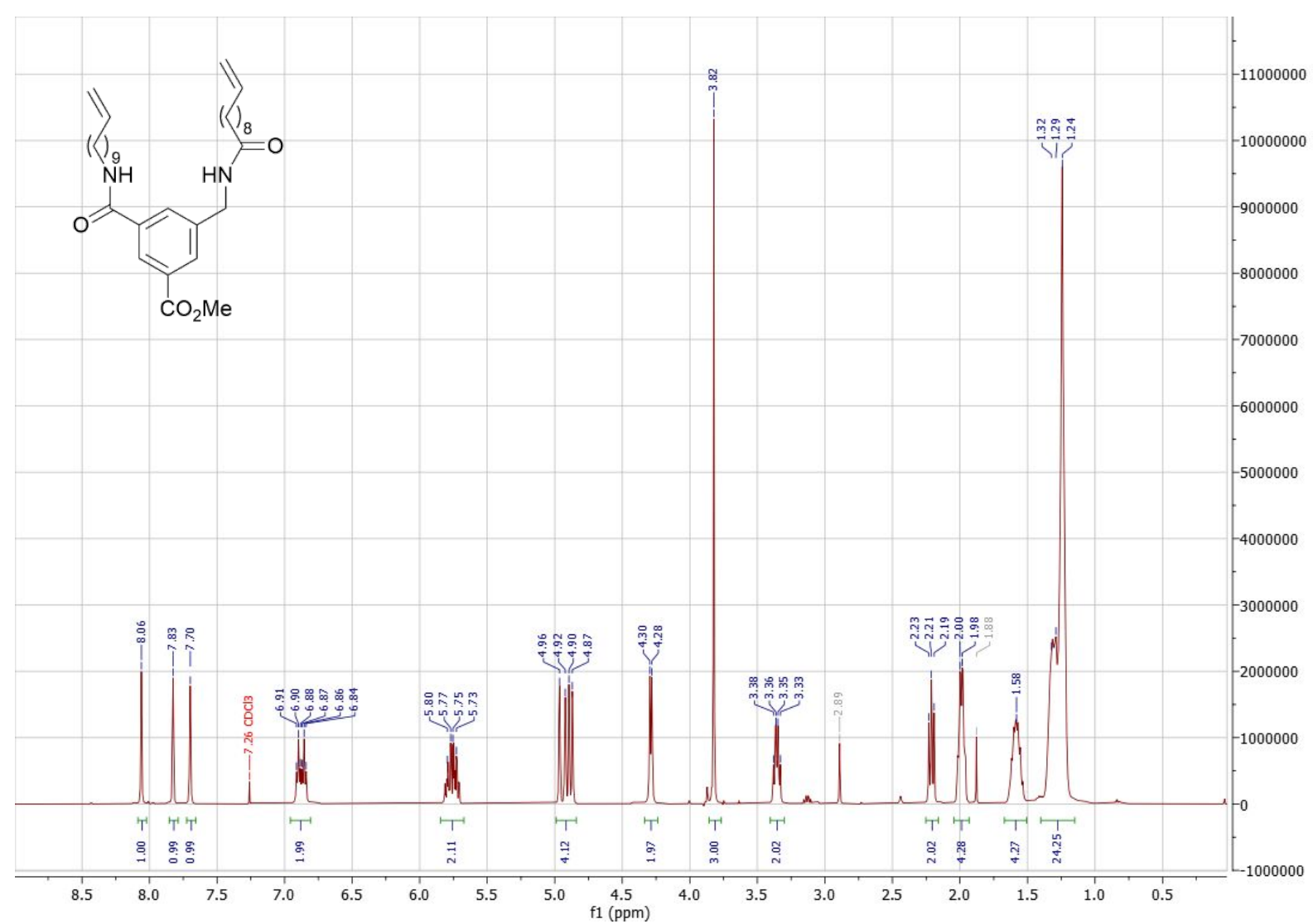

Figure S66. ${ }^{1} \mathrm{H}$ NMR spectrum of compound 29 in $\mathrm{CDCl}_{3}$ at $400 \mathrm{MHz}$.

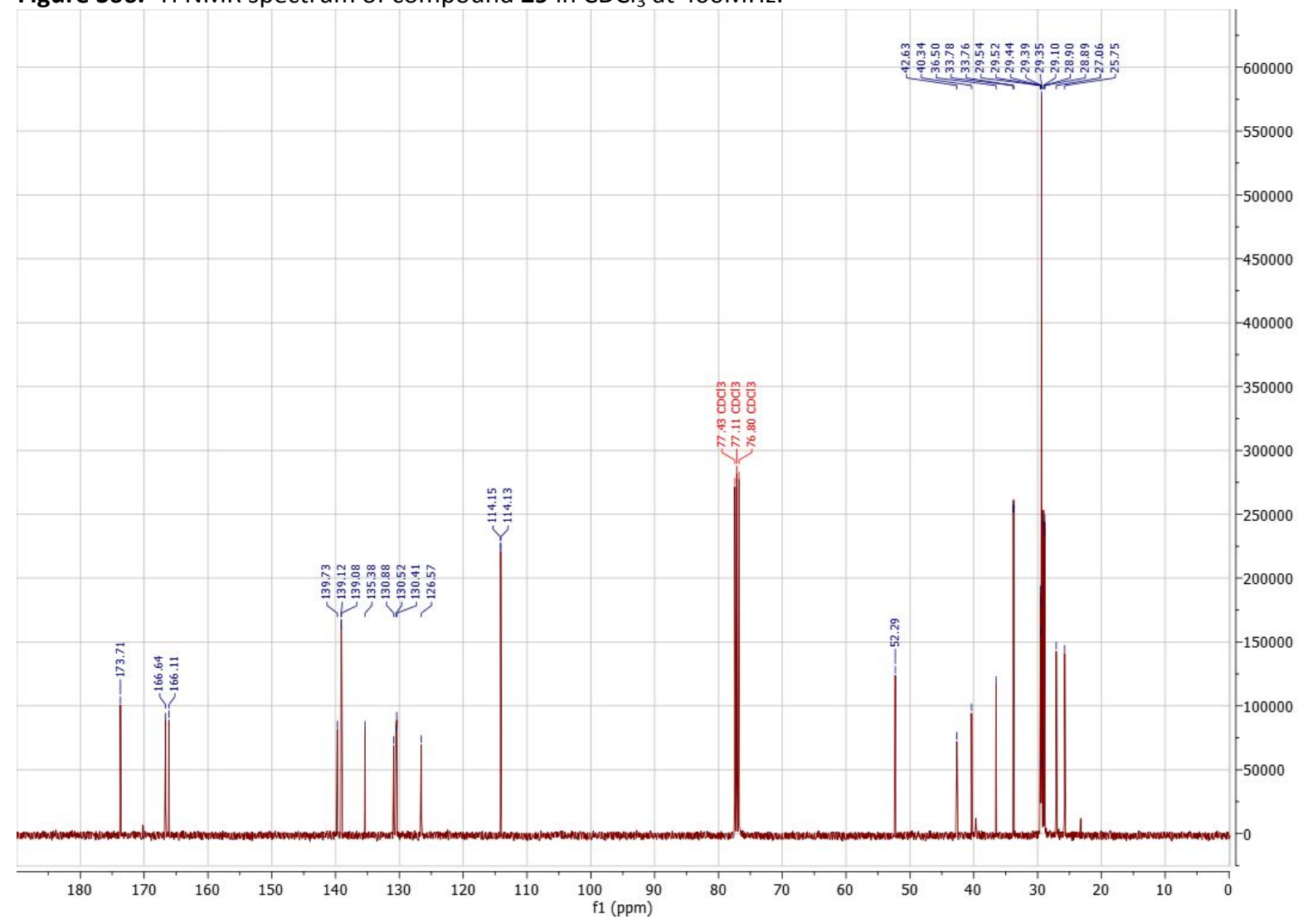

Figure S67. ${ }^{13} \mathrm{C}$ NMR spectrum of compound 29 in $\mathrm{CDCl}_{3}$ at $400 \mathrm{MHz}$. 


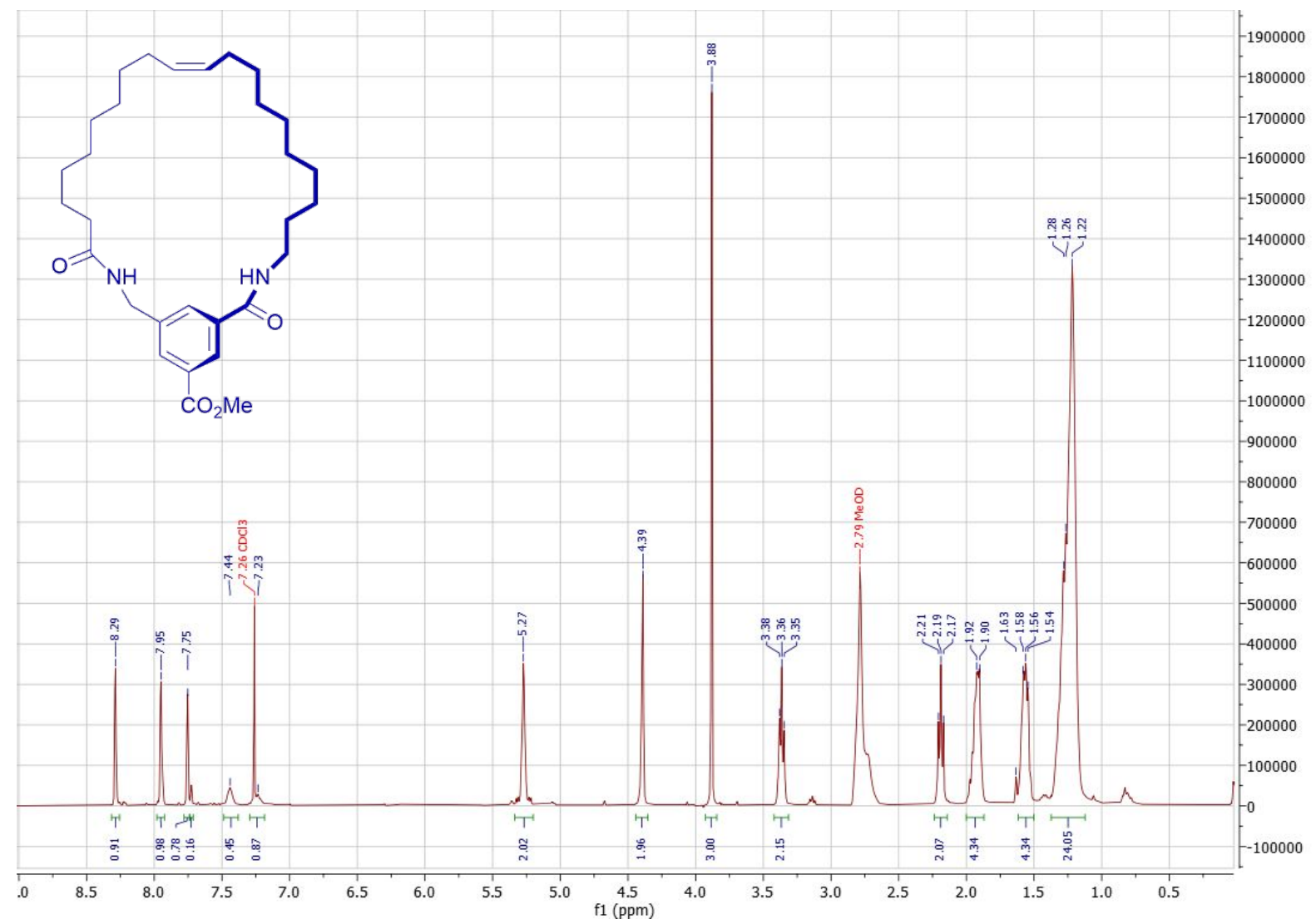

Figure S68. ${ }^{1} \mathrm{H}$ NMR spectrum of compound 30 in $\mathrm{CDCl}_{3}+\mathrm{CD}_{3} \mathrm{OD}$ at $400 \mathrm{MHz}$.

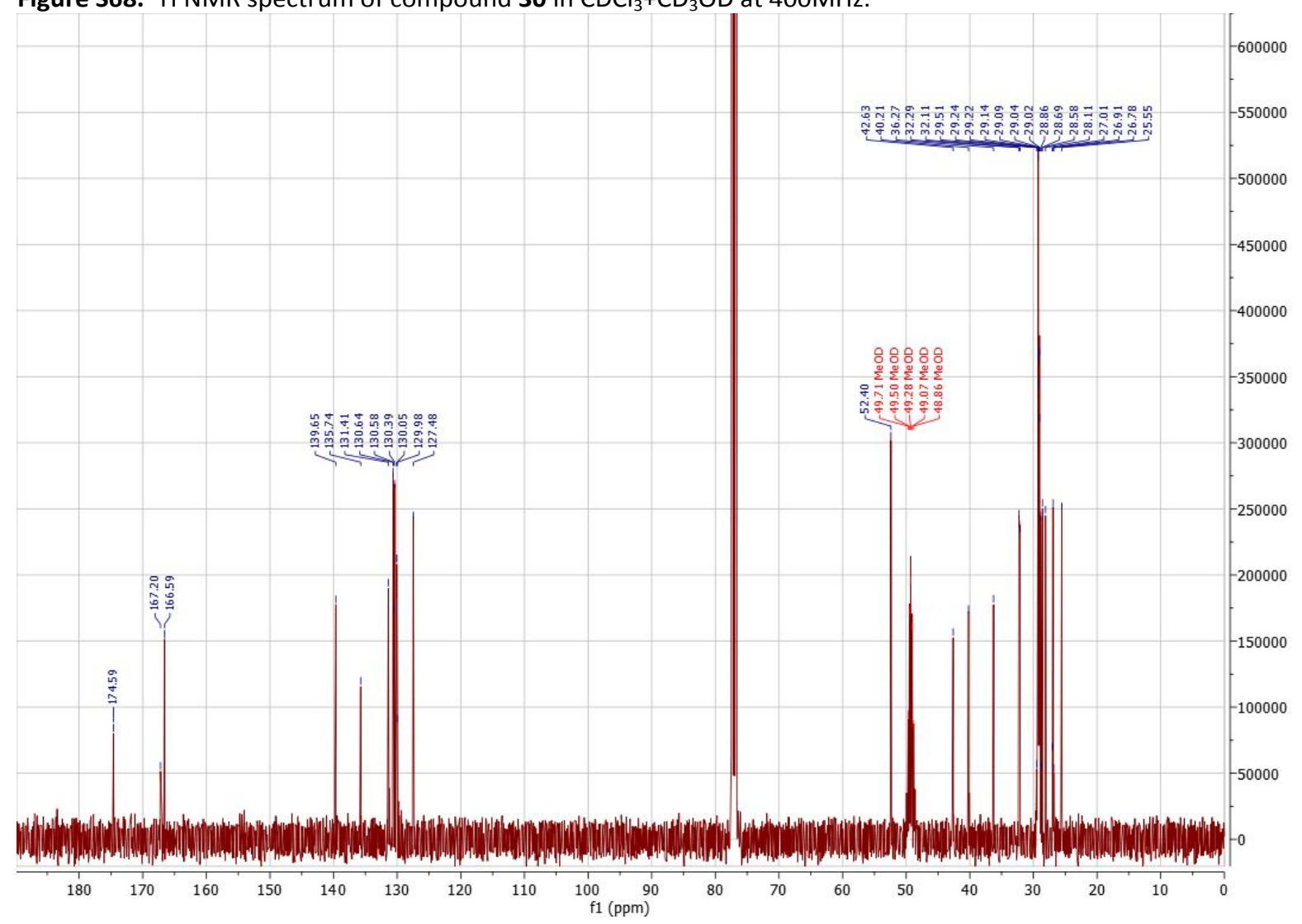

Figure S69. ${ }^{13} \mathrm{C}$ NMR spectrum of compound 30 in $\mathrm{CDCl}_{3}+\mathrm{CD}_{3} \mathrm{OD}$ at $400 \mathrm{MHz}$. 


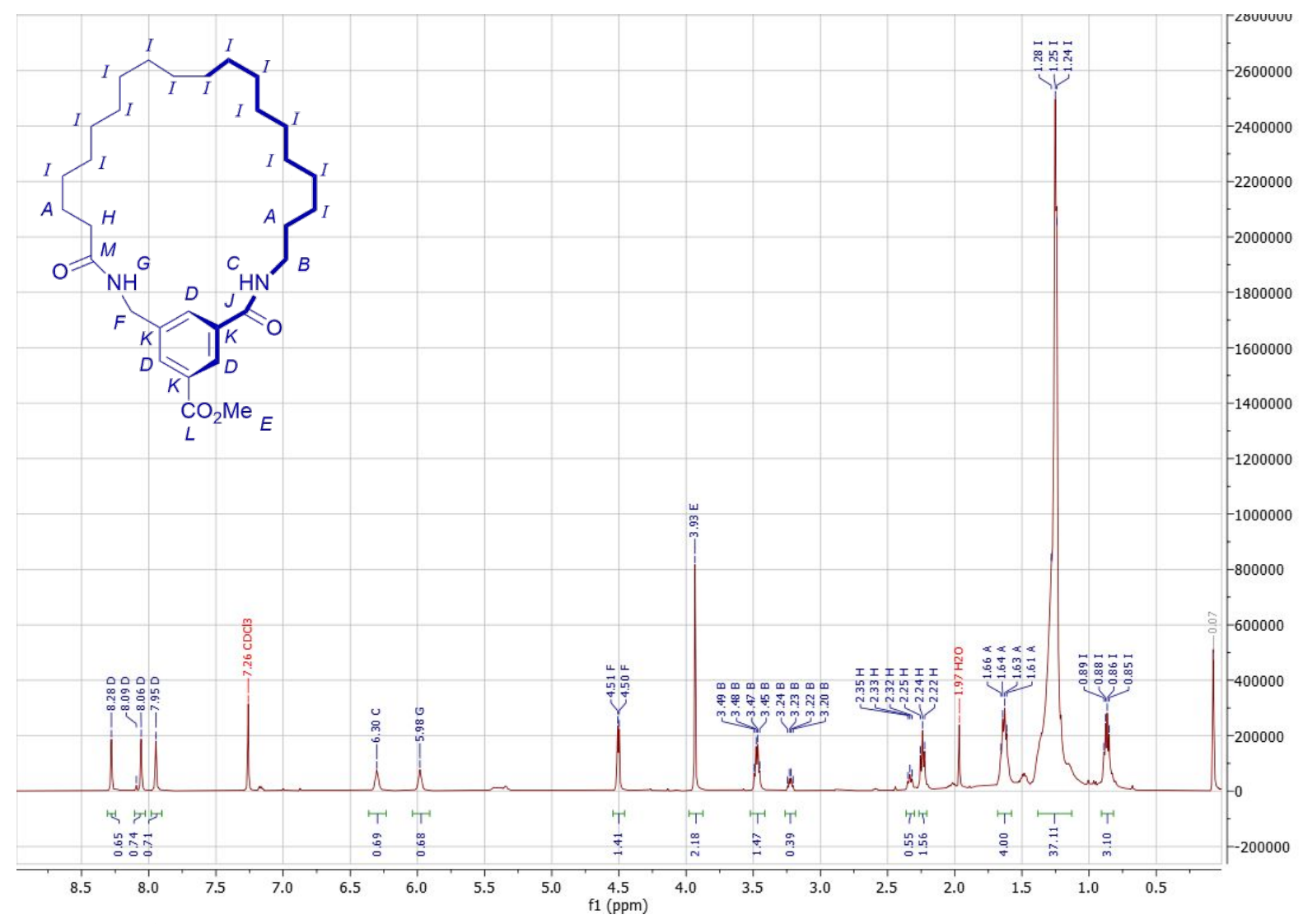

Figure S70. ${ }^{1} \mathrm{H}$ NMR spectrum of compound 31 in $\mathrm{CDCl}_{3}$ at $500 \mathrm{MHz}$.

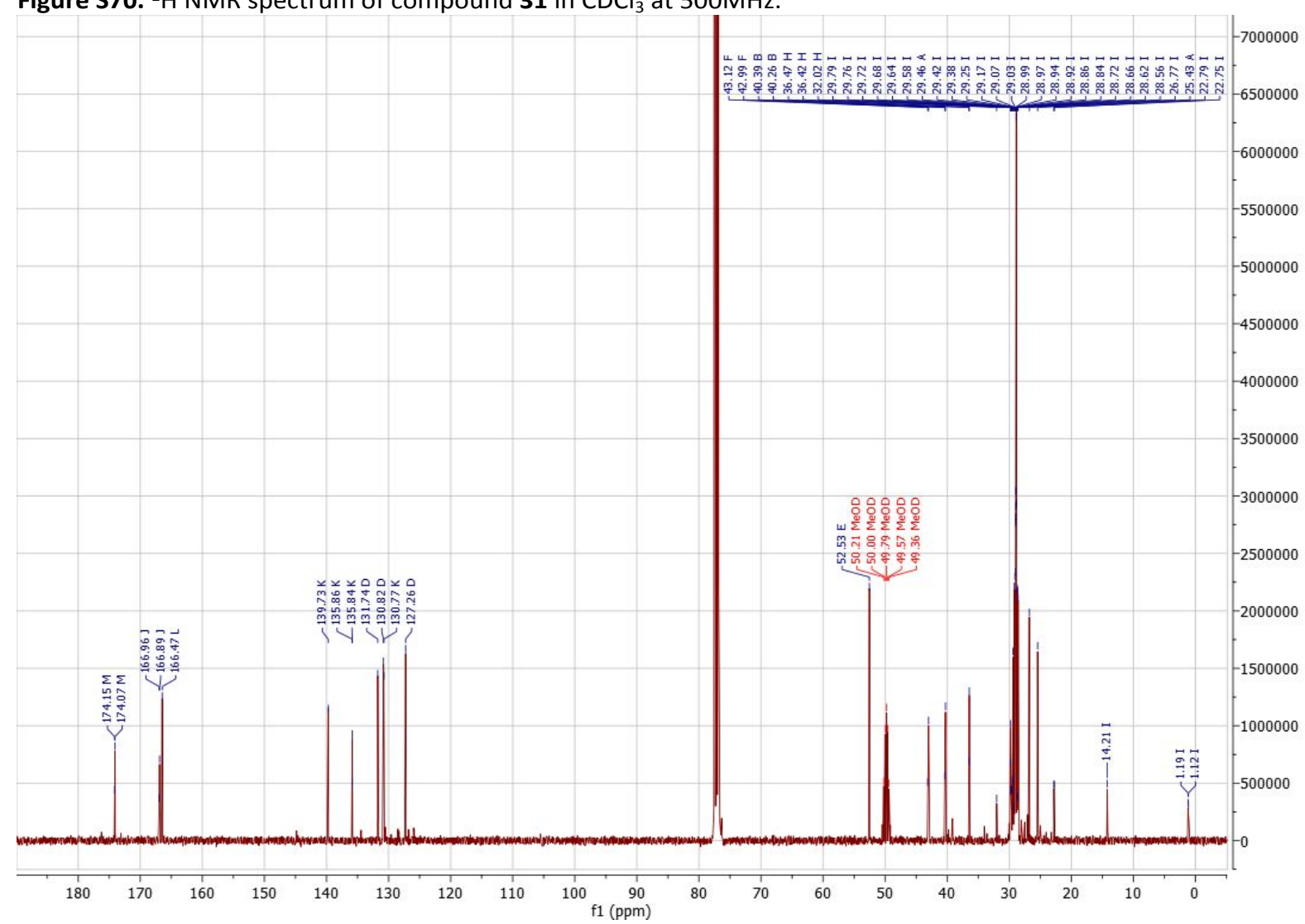

Figure S71. ${ }^{13} \mathrm{C}$ NMR spectrum of compound 31 in $\mathrm{CDCl}_{3}$ at $500 \mathrm{MHz}$. A drop of $\mathrm{CD}_{3} \mathrm{OD}$ was added for this measurement only to increase solubility, allowing a more concentrated sample. 


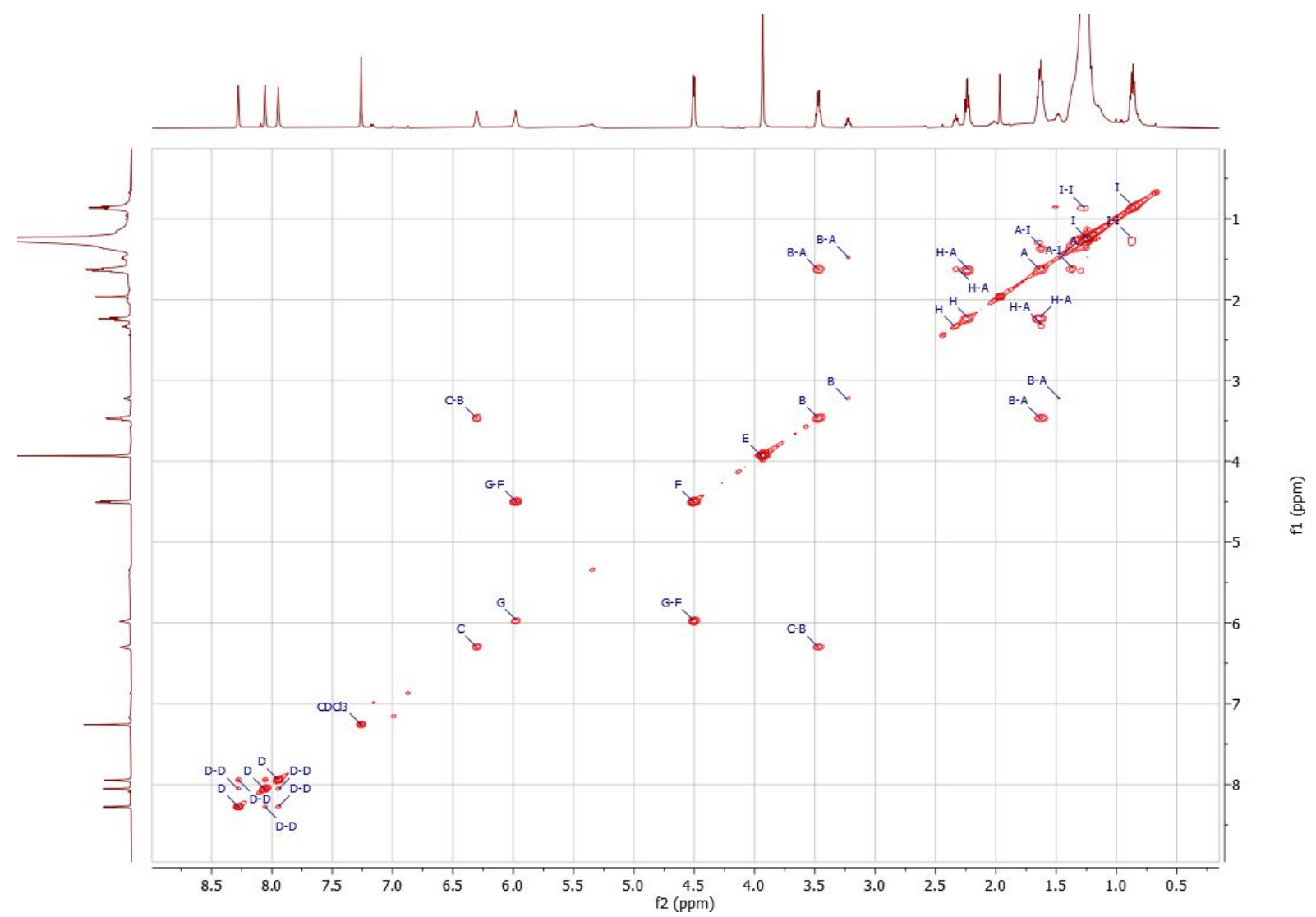

Figure S72. COSY NMR spectrum of compound 31 in $\mathrm{CDCl}_{3}$ at $500 \mathrm{MHz}$.

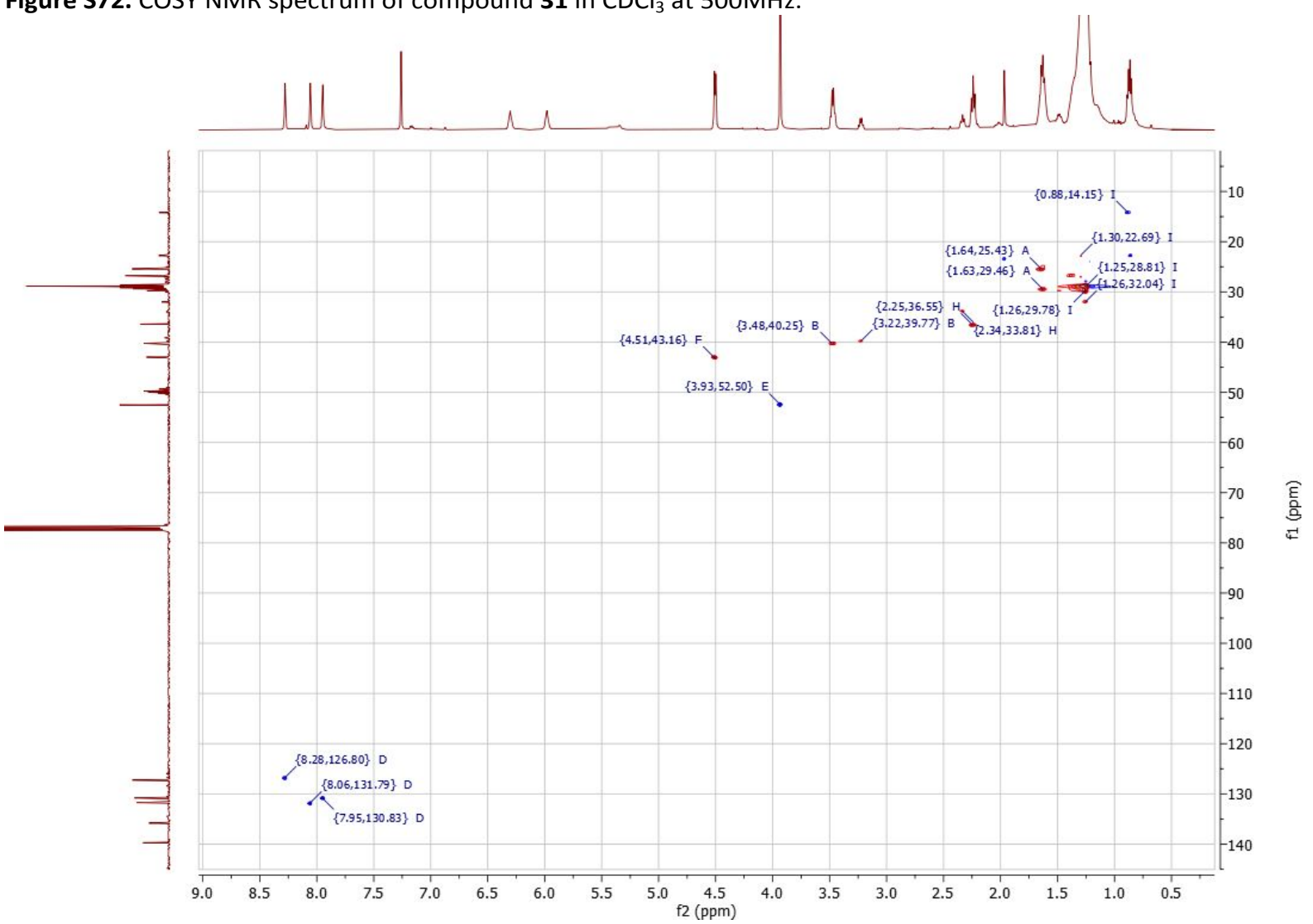

Figure S73. HSQC NMR spectrum of compound 31 in $\mathrm{CDCl}_{3}$ at $500 \mathrm{MHz}$. 


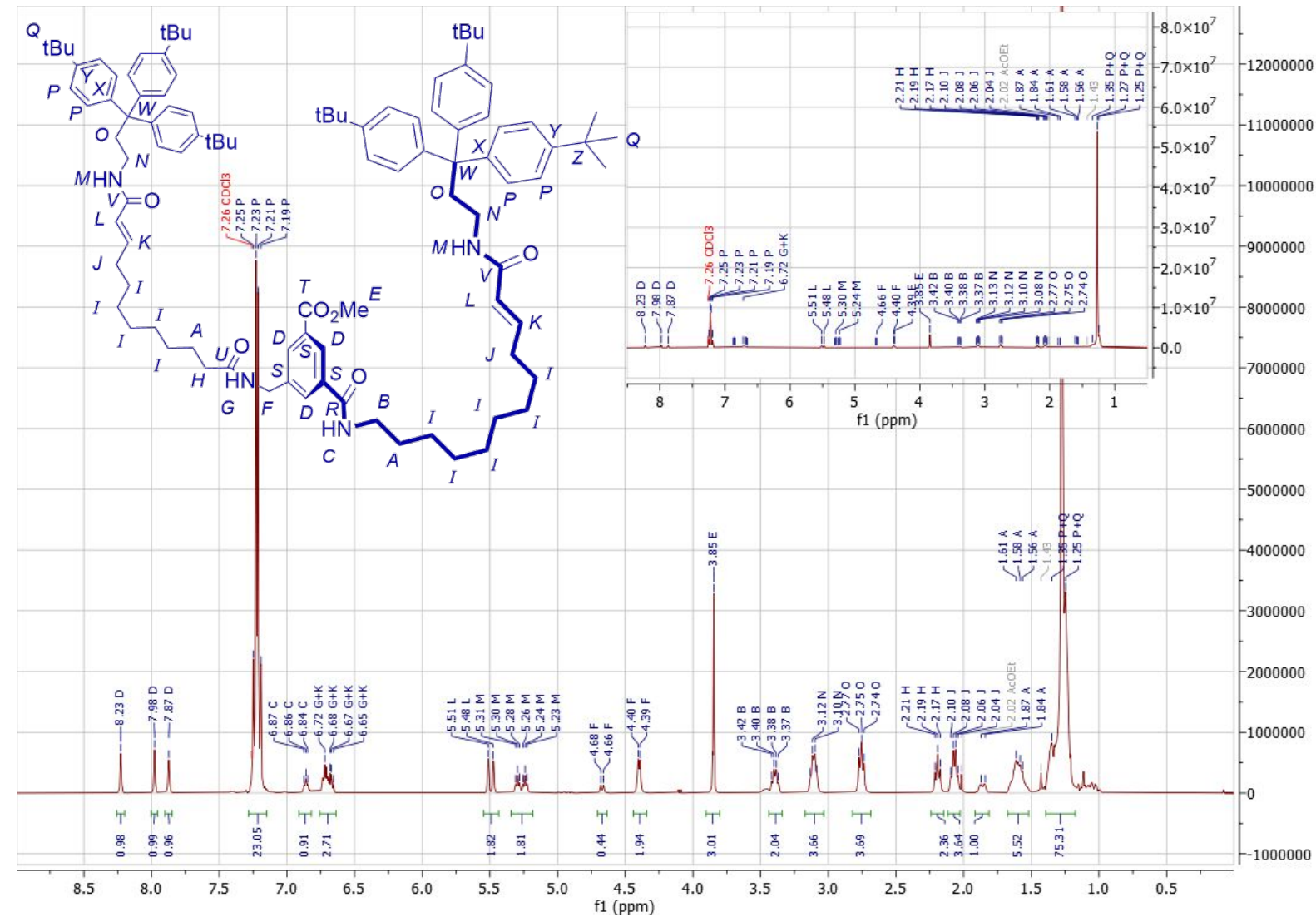

Figure S74. ${ }^{1} \mathrm{H}$ NMR spectrum of compound 32 in $\mathrm{CDCl}_{3}$ at $500 \mathrm{MHz}$.

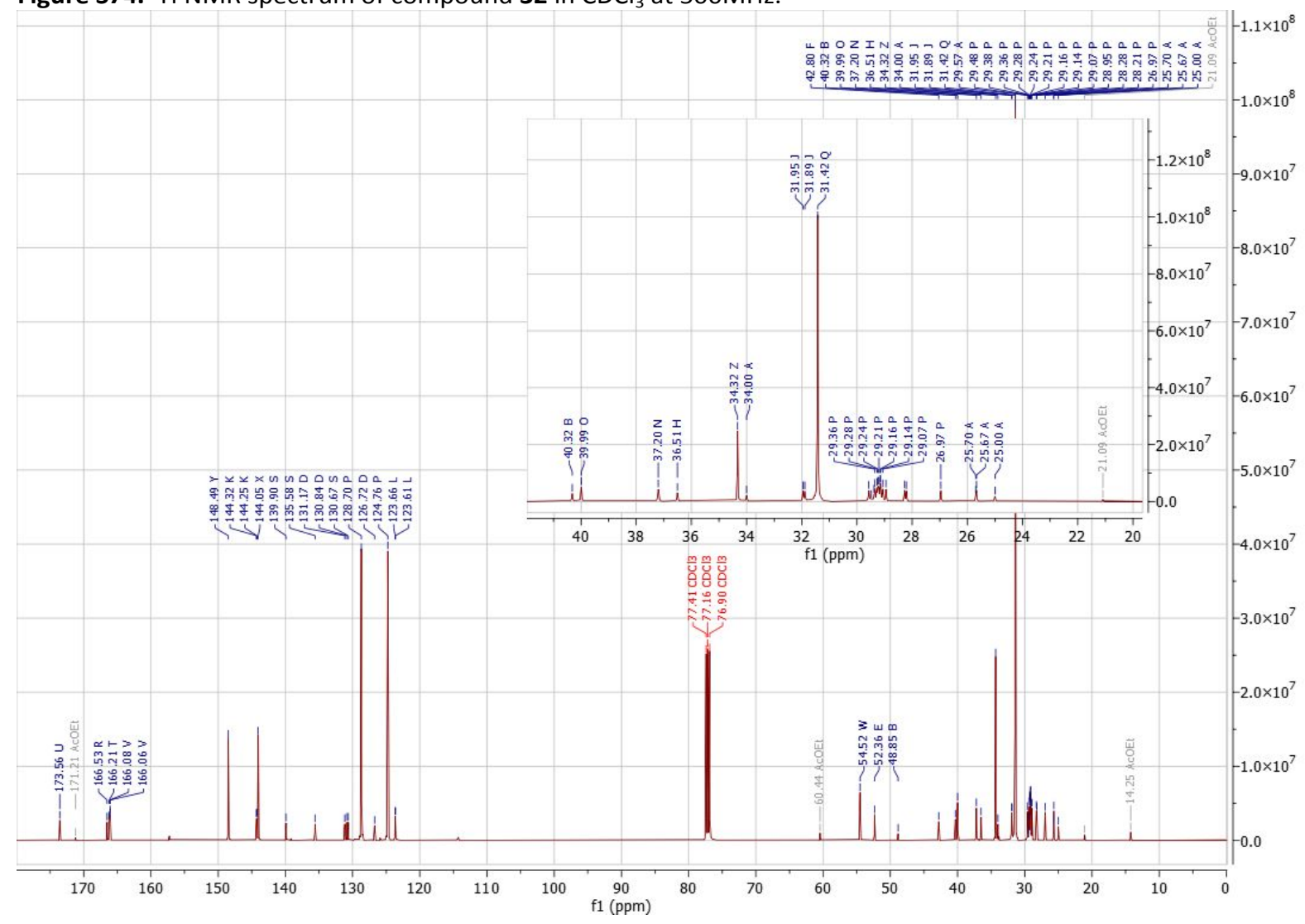

Figure S75. ${ }^{13} \mathrm{C}$ NMR spectrum of compound 32 in $\mathrm{CDCl}_{3}$ at $500 \mathrm{MHz}$. 


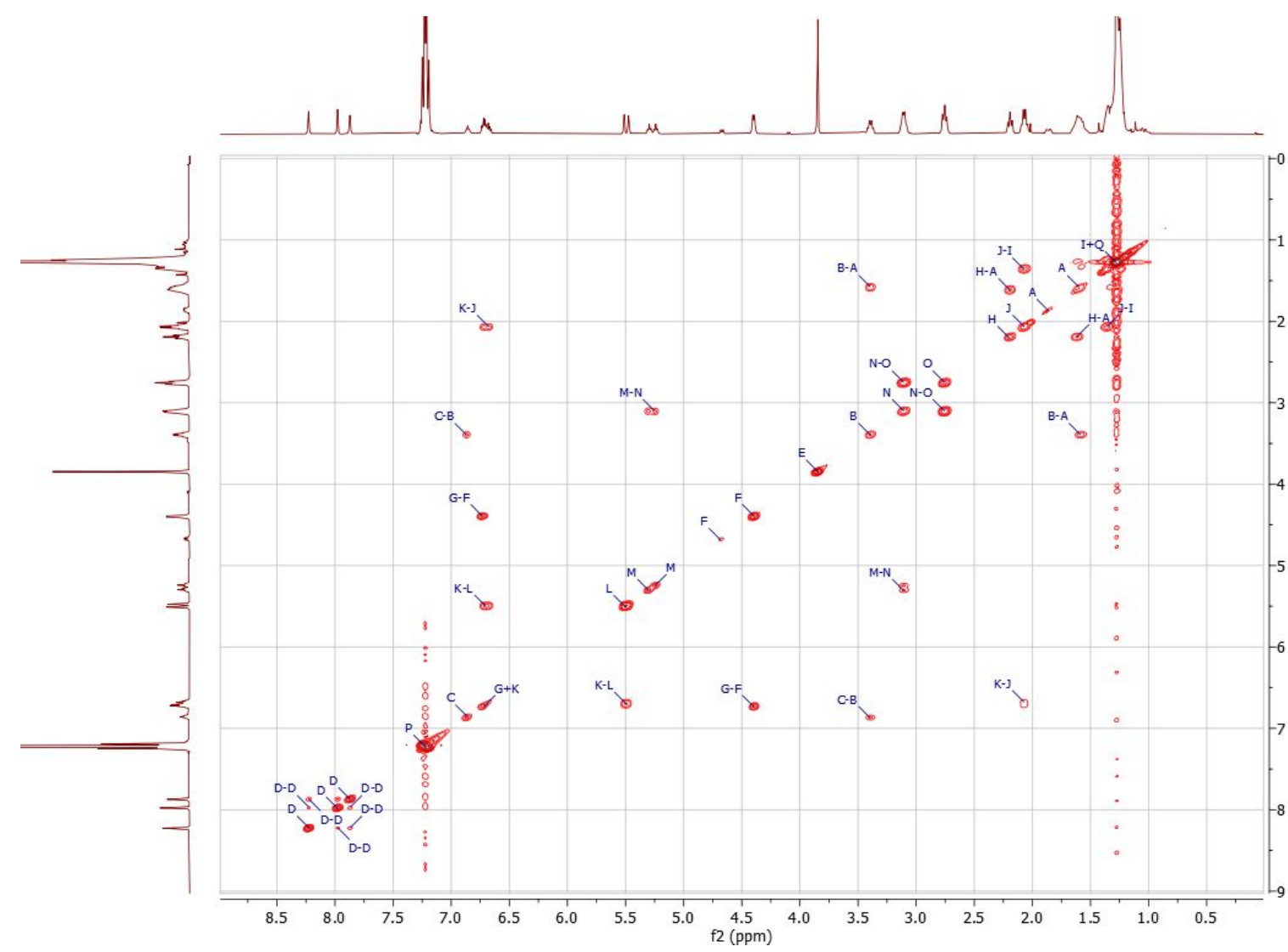

Figure S76. COSY NMR spectrum of compound 32 in $\mathrm{CDCl}_{3}$ at $500 \mathrm{MHz}$.

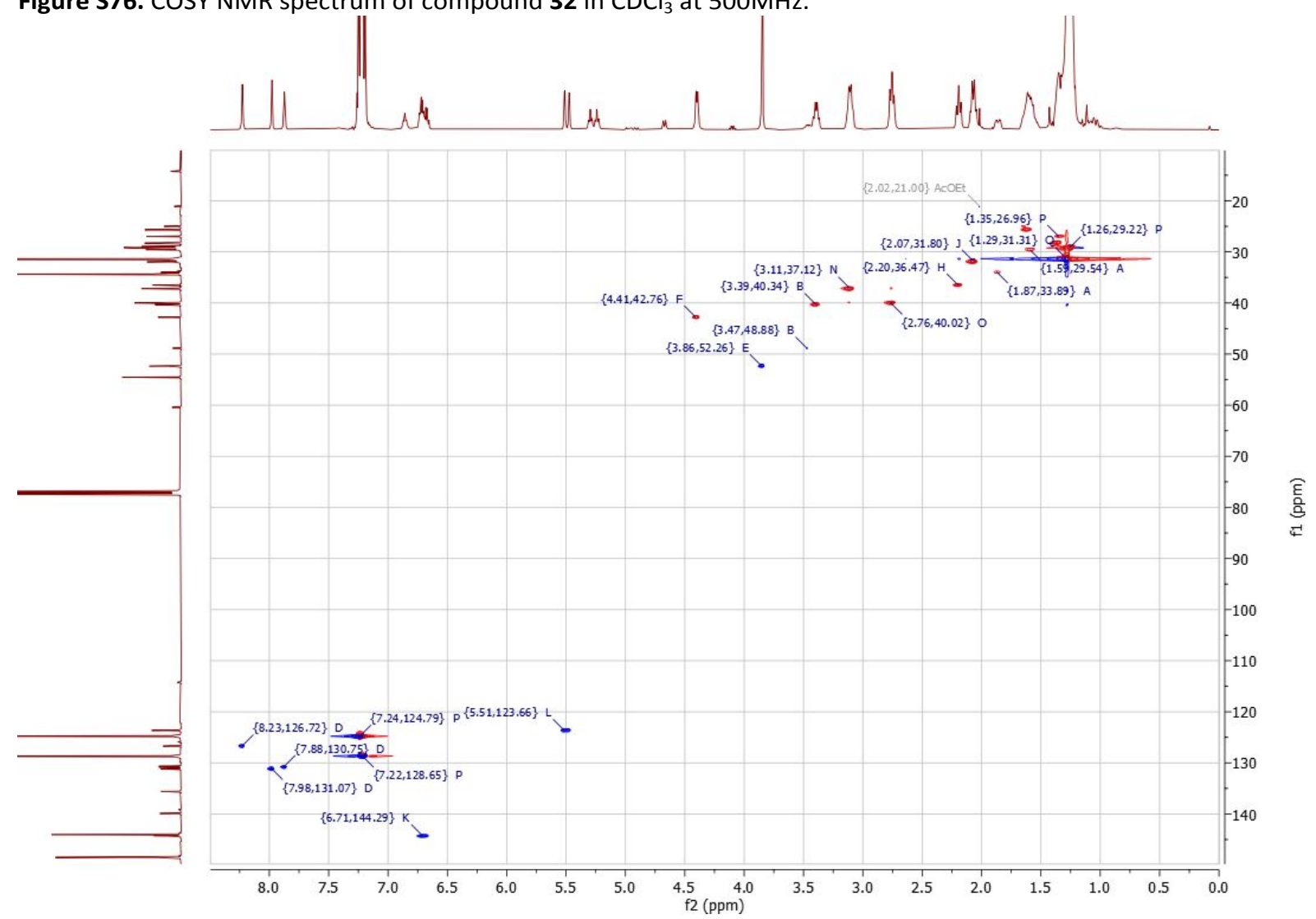

Figure S77. HSQC NMR spectrum of compound 32 in $\mathrm{CDCl}_{3}$ at $500 \mathrm{MHz}$. 


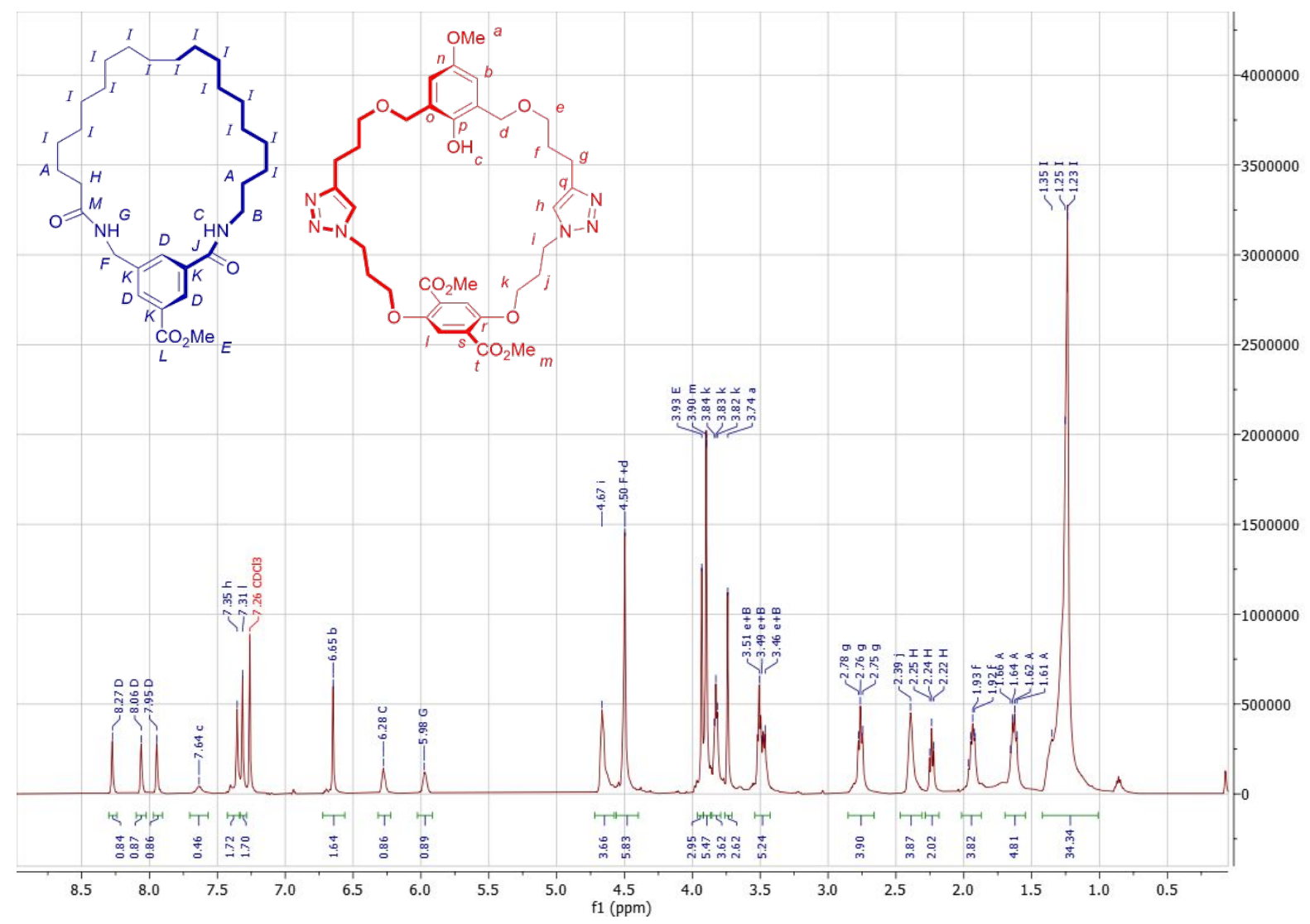

Figure S78. ${ }^{1} \mathrm{H}$ NMR spectrum of an equimolar mixture of macrocycles $\mathbf{2 6}$ and $\mathbf{3 1}$ in $\mathrm{CDCl}_{3}$ at $500 \mathrm{MHz}$.

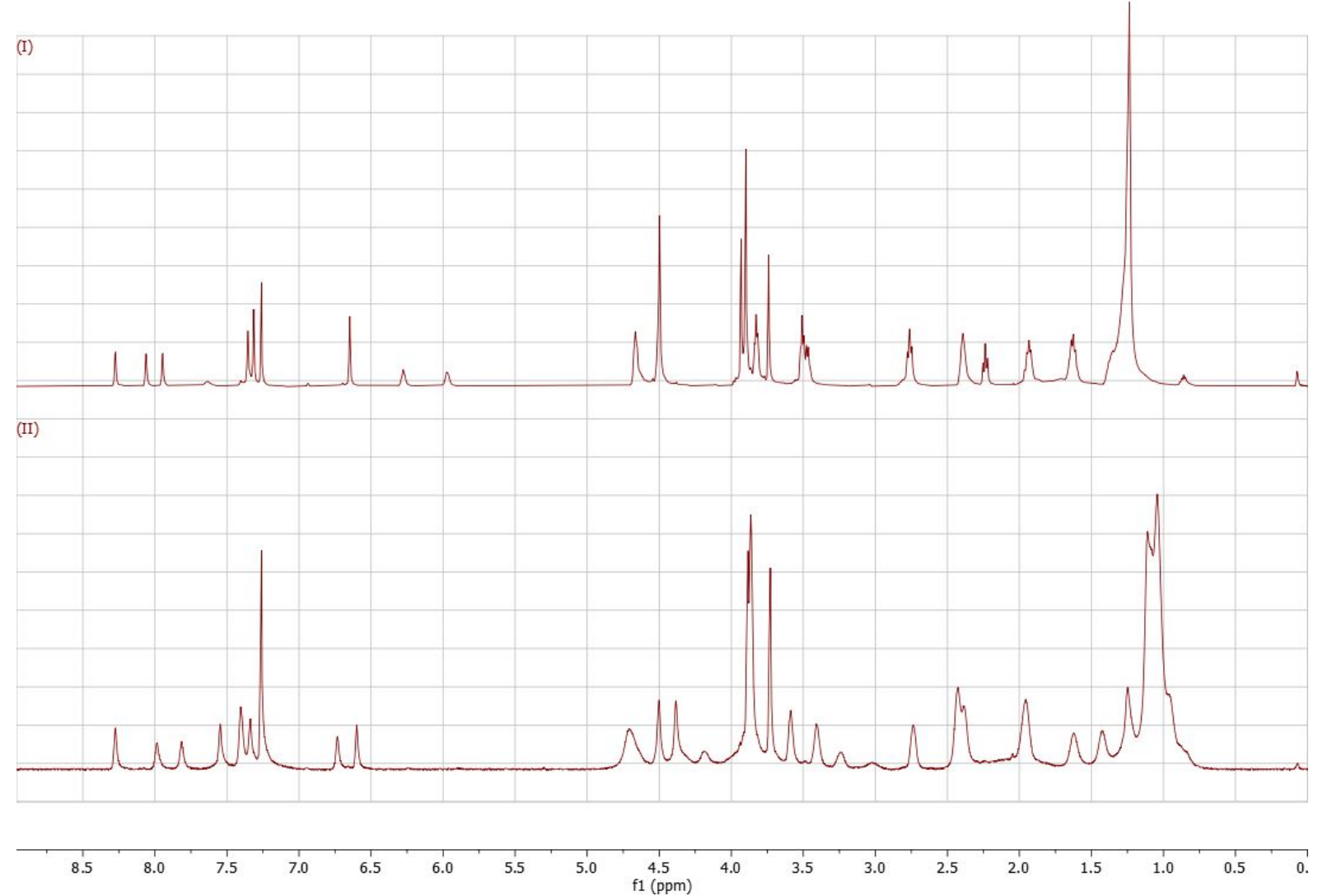

Figure S79. Comparison between ${ }^{1} \mathrm{H}$ NMR spectra of an equimolar mixture of macrocycles 26 and 31 (I) and [2]catenane 2 (II) in $\mathrm{CDCl}_{3}$ at $500 \mathrm{MHz}$. 


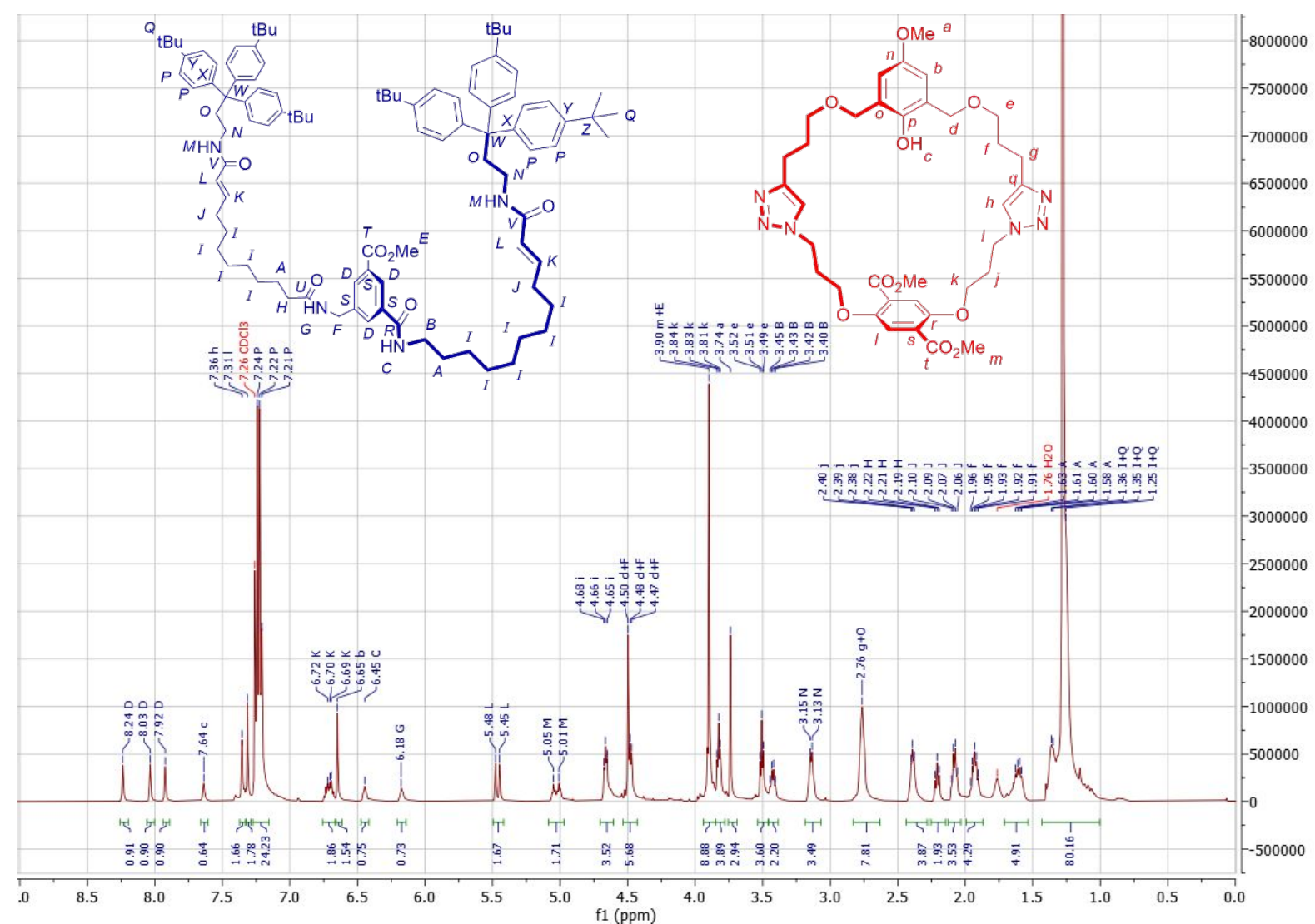

Figure S80. ${ }^{1} \mathrm{H}$ NMR spectrum of an equimolar mixture of macrocycle 26 and thread 32 in $\mathrm{CDCl}_{3}$ at $500 \mathrm{MHz}$.

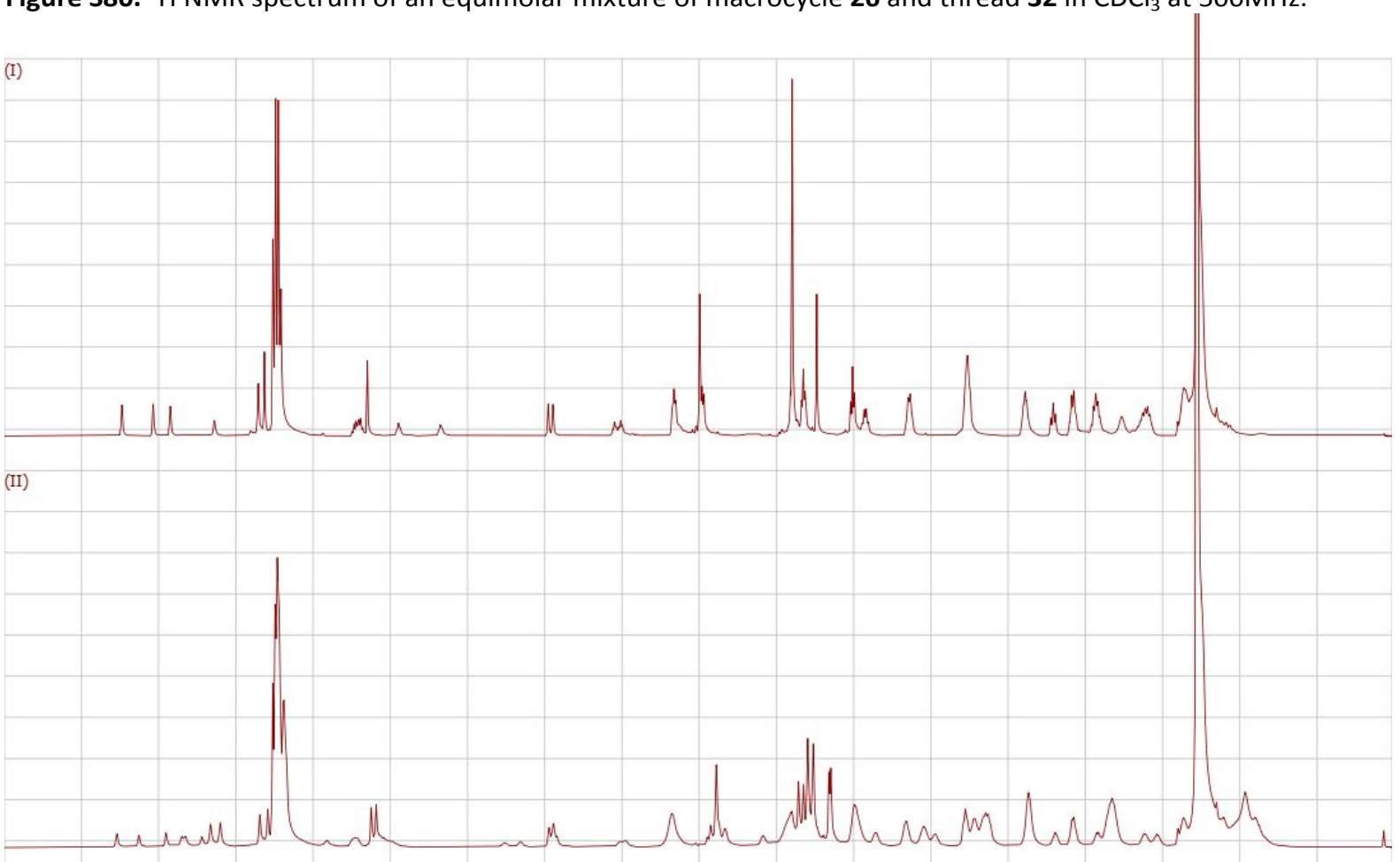

$\begin{array}{lllllllllllllllll}8.5 & 8.0 & 7.5 & 7.0 & 6.5 & 6.0 & 5.5 & 5.0 & \begin{array}{c}1 \\ \mathrm{f} 1(\mathrm{ppm})\end{array} & 4.0 & 3.5 & 3.0 & 2.5 & 2.0 & 1.5 & 1.0 & 0.5\end{array}$

Figure S81. Comparison between ${ }^{1} \mathrm{H}$ NMR spectra of an equimolar mixture of macrocycle 26 and thread 32 (I) and [2] rotaxane 3 (II) in $\mathrm{CDCl}_{3}$ at $500 \mathrm{MHz}$. 


\section{Mass Spectra}
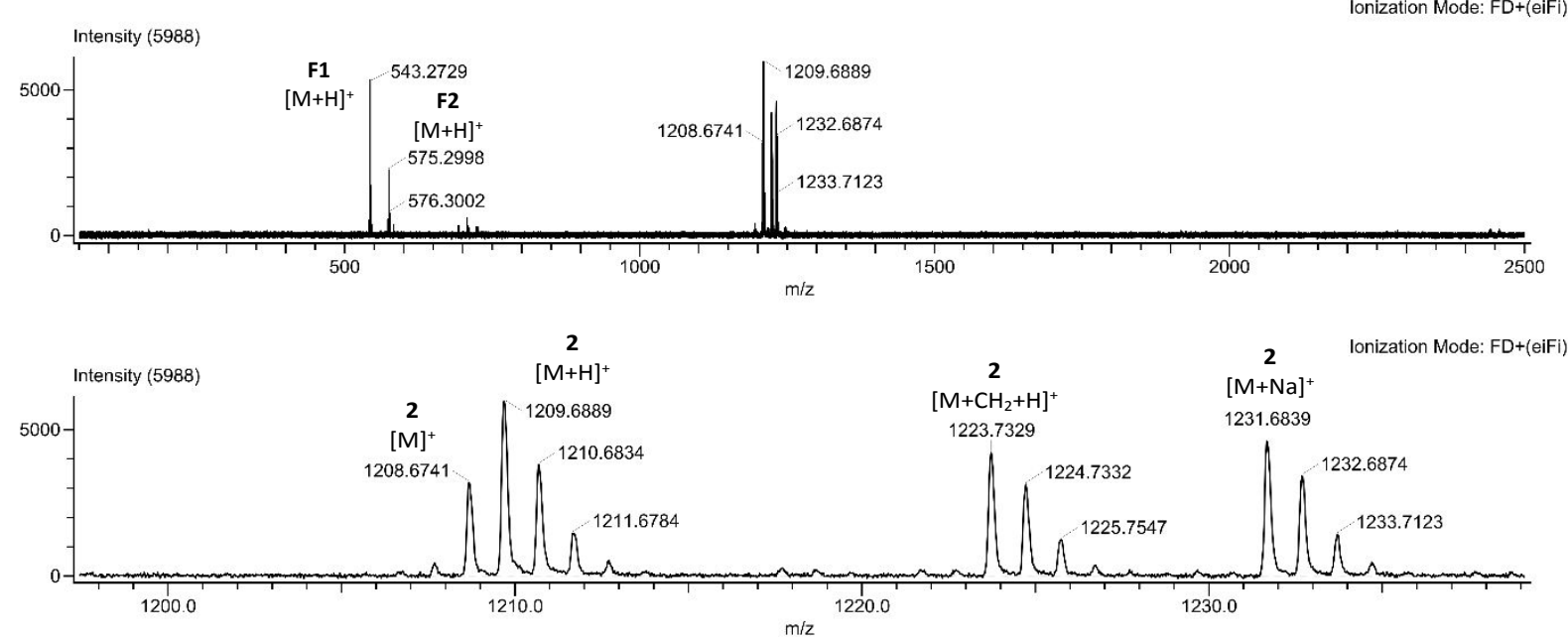

Figure S82. Mass spectrum (FD+) of [2]catenane 2. The ions labelled by F1 and F2 (Figure S82) most likely arise by ionization of species formed by thermal decomposition of the parent compound $\mathbf{2}$ during the FD process.

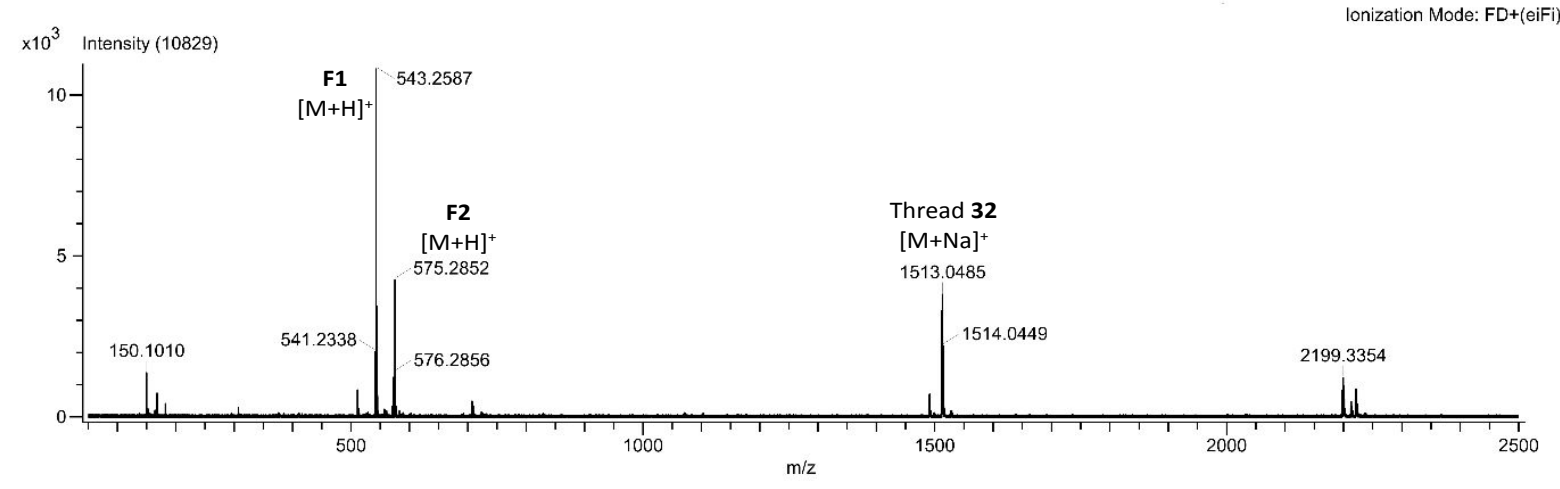

Ionization Mode: $\mathrm{FD}+(\mathrm{eiFi})$

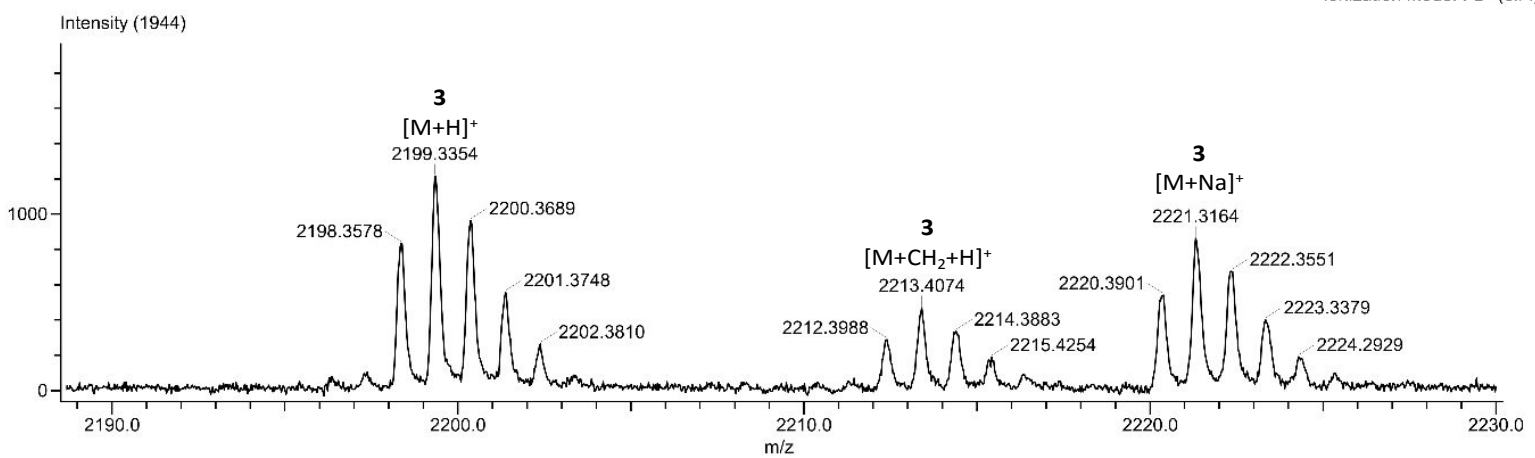

Figure 583. Mass spectrum ( $\mathrm{FD}^{+}$) of [2]rotaxane 3. The ions labelled by F1 and F2 most likely arise by ionization of species formed by decomposition of the parent compound $\mathbf{3}$ during the FD process. The methylene adduct could indicate a byproduct formed during the cross metathesis reaction.
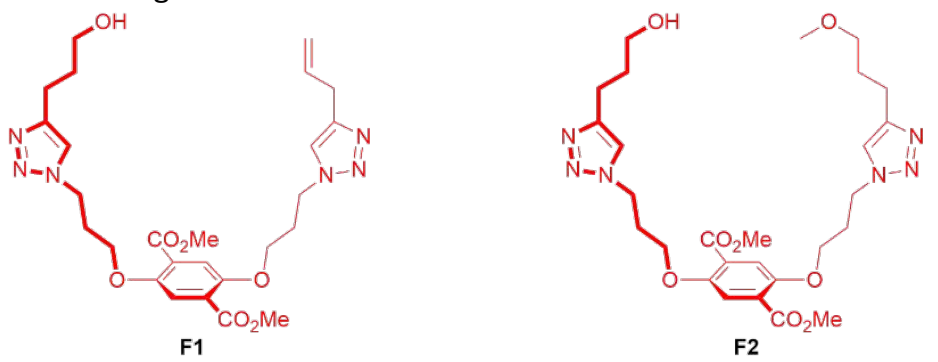

Figure S84. Proposed structures of macrocycle decomposition products F1 and F2. 

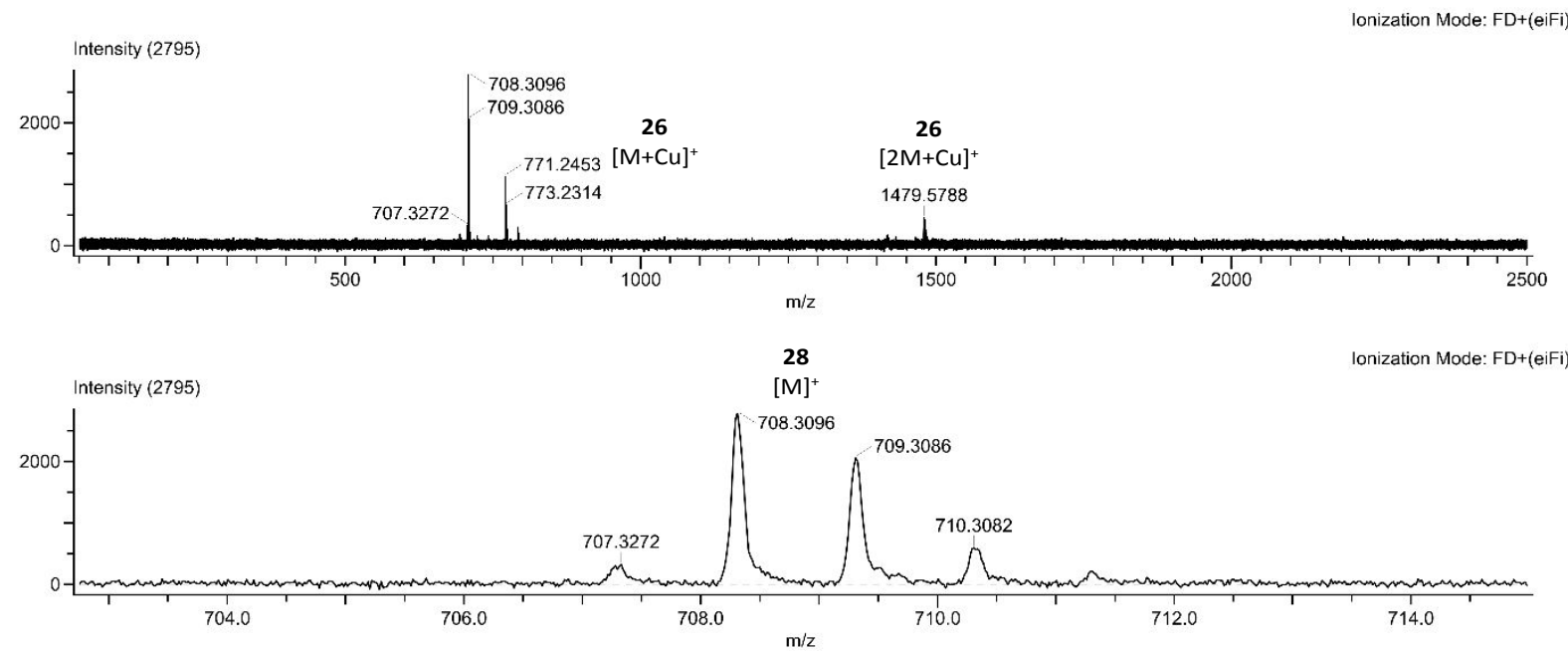

Figure S85. Mass spectrum $\left(\mathrm{FD}^{+}\right)$of macrocycle 26.

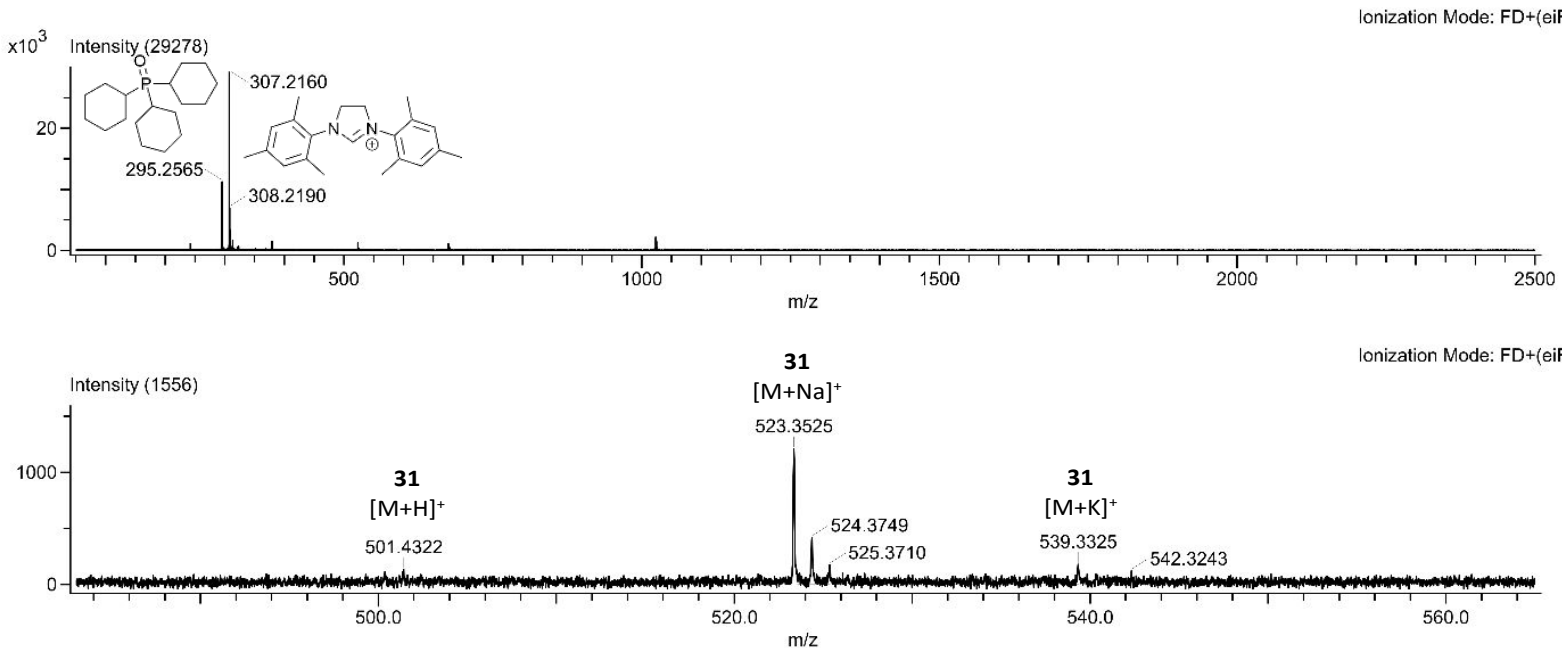

Figure S86. Mass spectrum $\left(\mathrm{FD}^{+}\right)$of macrocycle 31. The prominent impurities are due to traces of Grubbs $2^{\text {nd }}$ generation catalyst, used in the synthesis of this compound. 

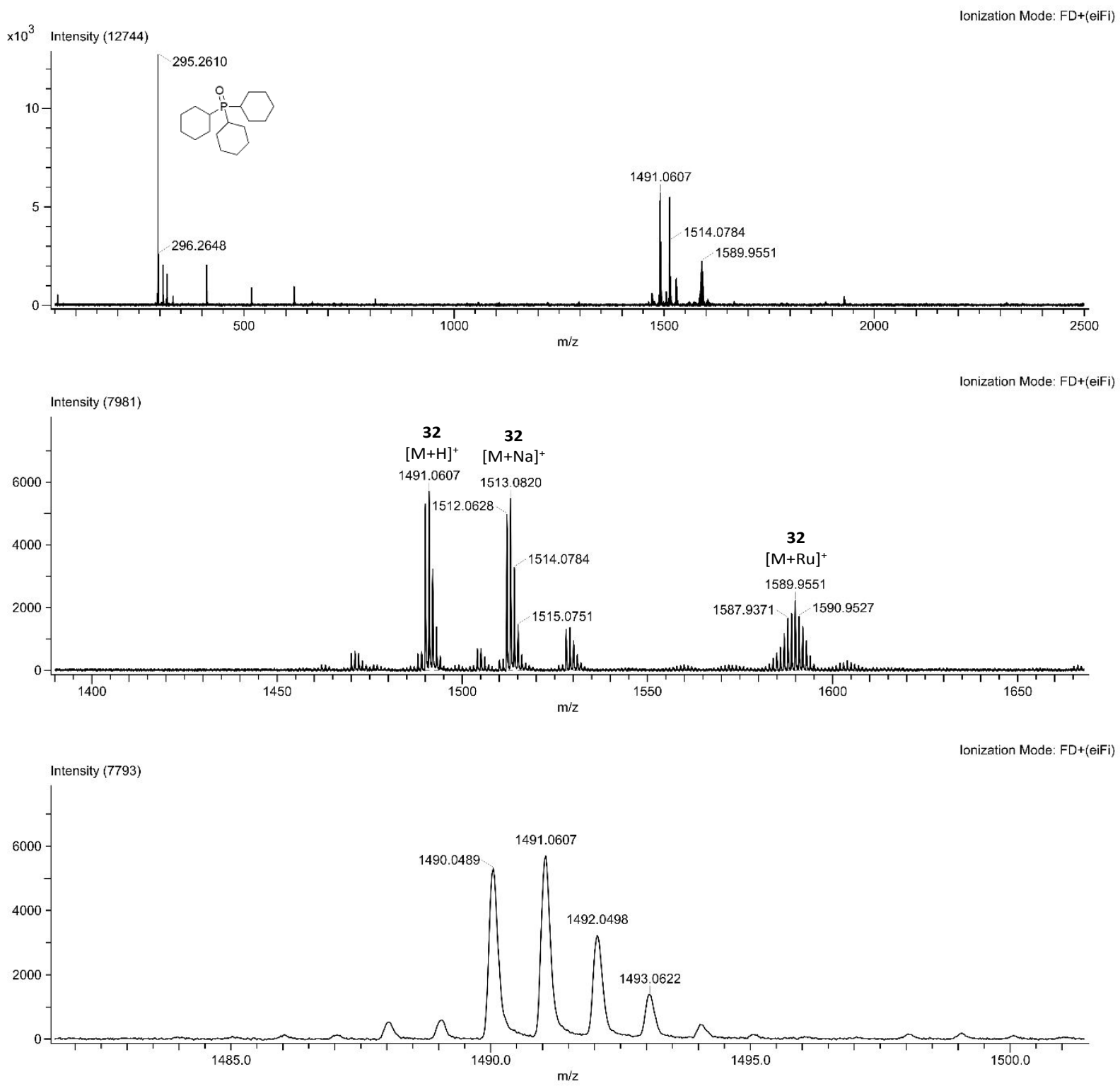

Figure S87. Mass spectrum (FD') of thread 32. 


\section{DFT Calculations}

DFT geometry optimizations were performed at the PM6/6-31G* level of theory using the Gaussian 09 (revision D01) program suite..$^{8,9}$
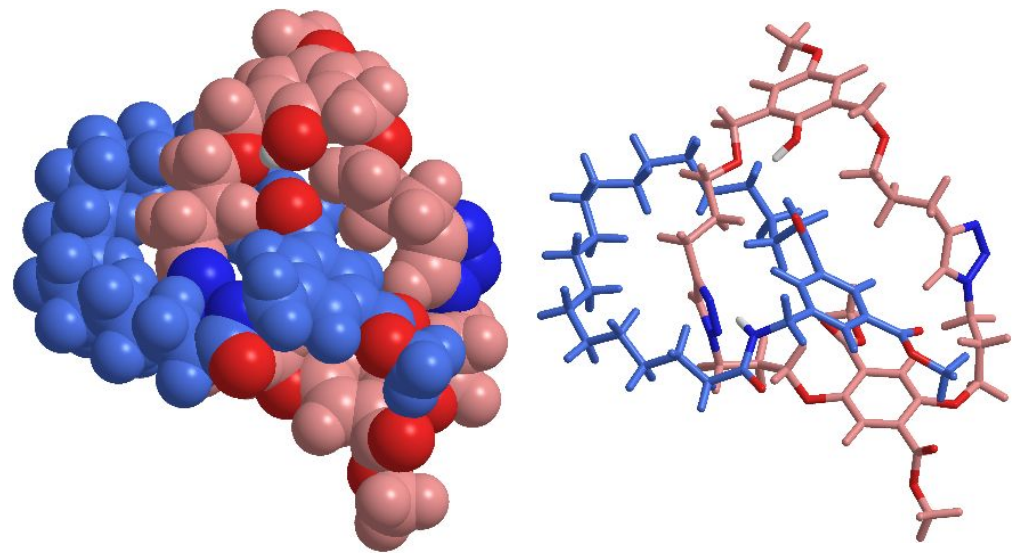

Figure S88. Front view of space filling (left) and tube (right) representations of the calculated structure of [2]catenane 2. $\mathrm{C}$ and $\mathrm{H}$ atoms have been colored light blue or light red based on the parent fragment.
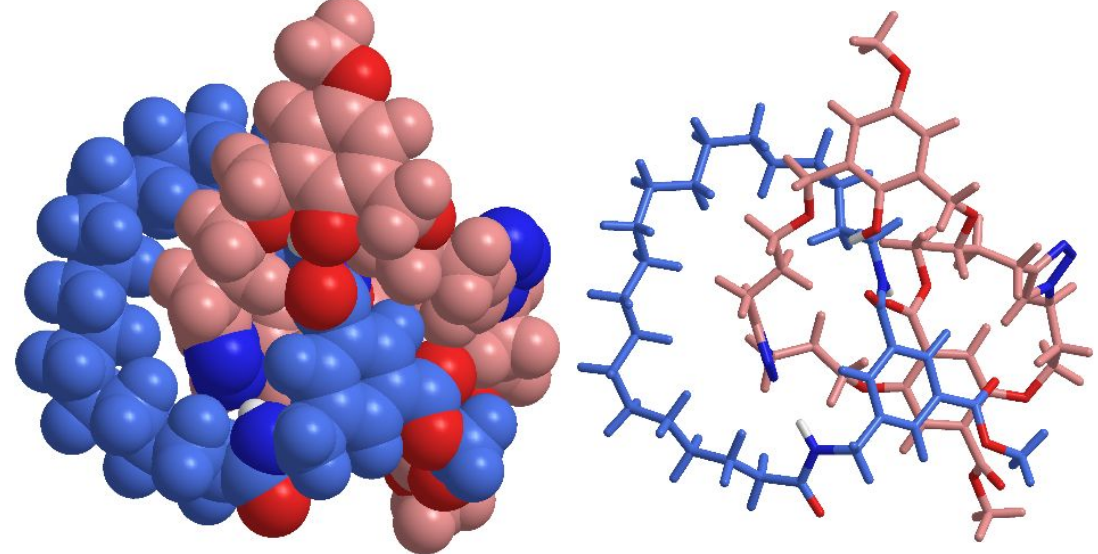

Figure S89. Top view of space filling (left) and tube (right) representations of the calculated structure of [2]catenane 2. $\mathrm{C}$ and $\mathrm{H}$ atoms have been colored light blue or light red based on the parent fragment.

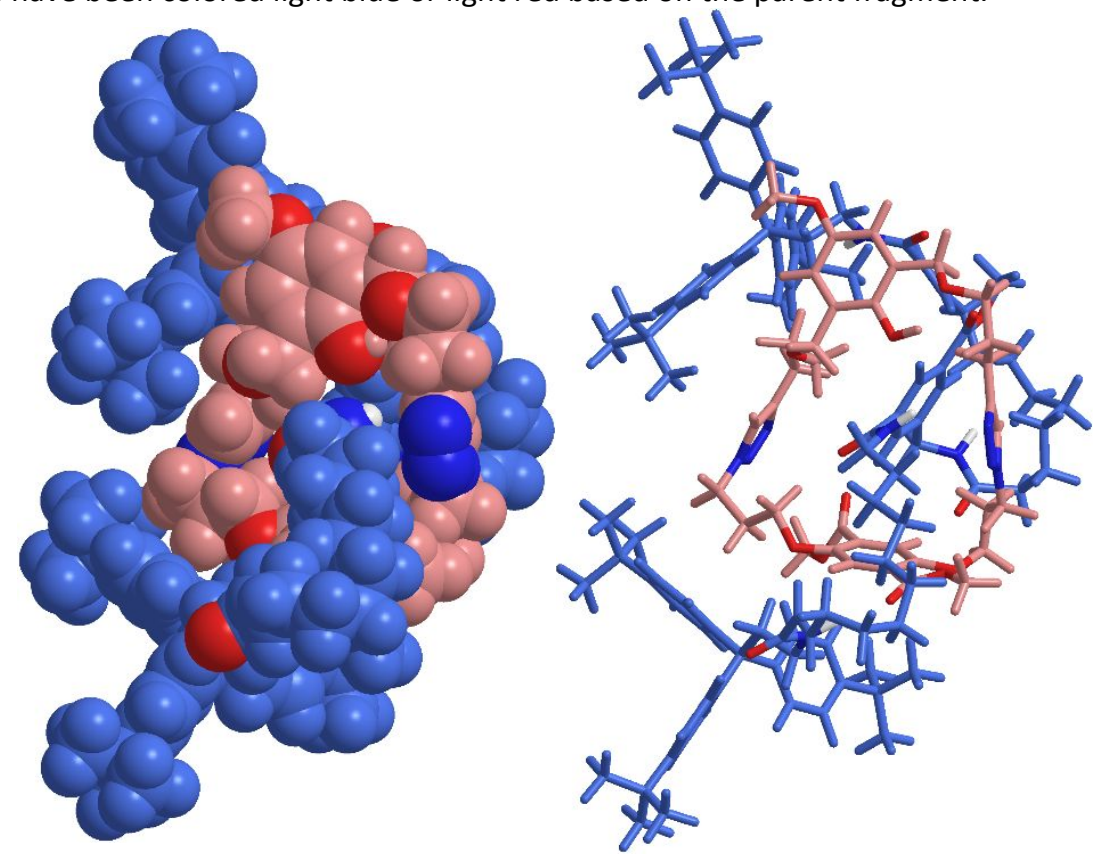

Figure 590. Front view of space filling (left) and tube (right) representations of the calculated structure of [2] rotaxane 3. $\mathrm{C}$ and $\mathrm{H}$ atoms have been colored light blue or light red based on the parent fragment. 


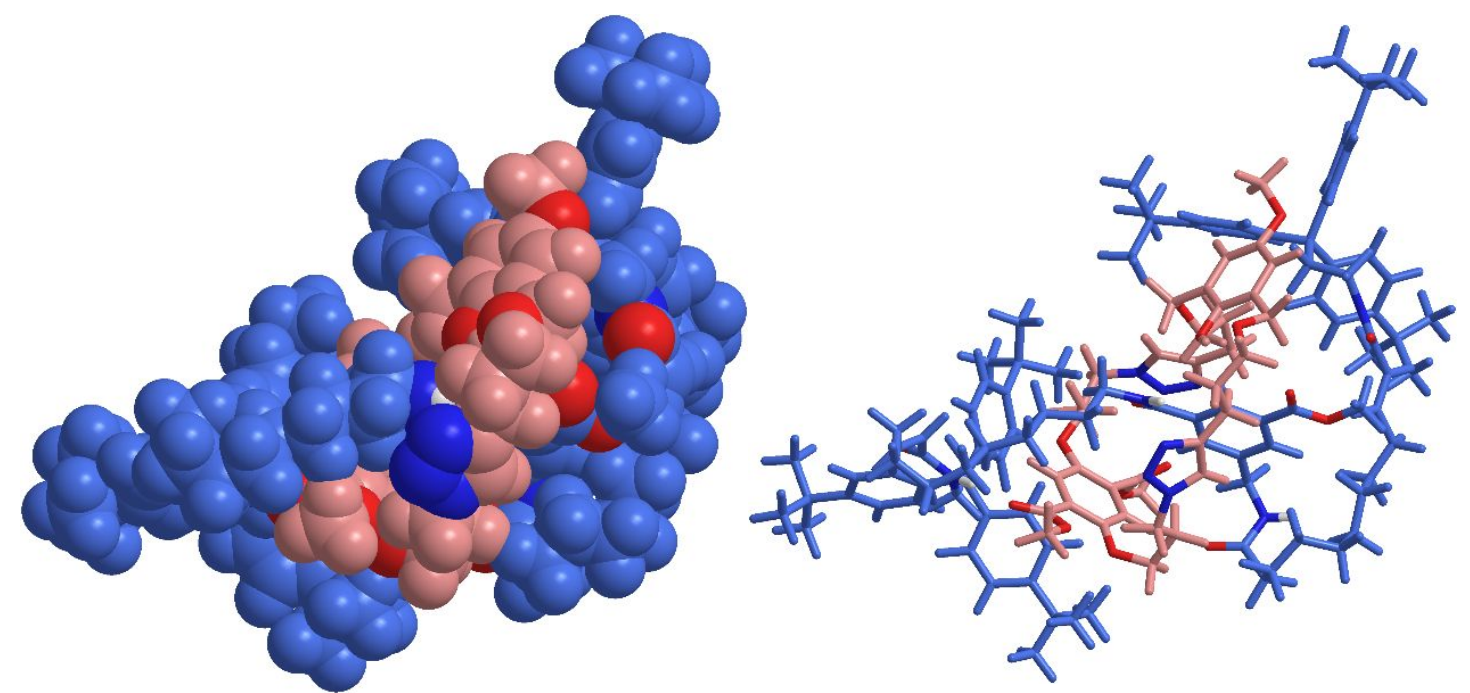

Figure S91. Side view of space filling (left) and tube (right) representations of the calculated structure of [2] rotaxane 3. $\mathrm{C}$ and $\mathrm{H}$ atoms have been colored light blue or light red based on the parent fragment.

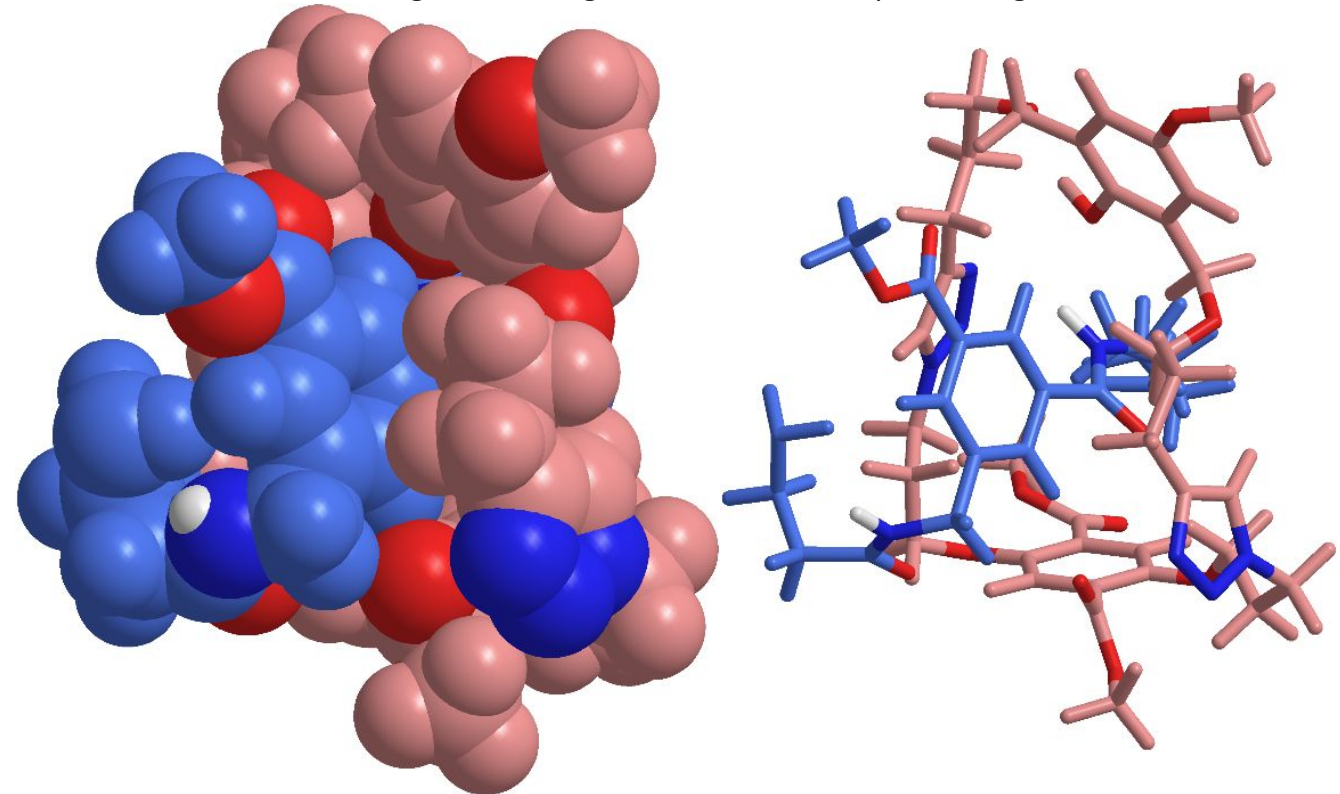

Figure S92. Front view of space filling (left) and tube (right) representations of the calculated structure of [2]rotaxane 3. $\mathrm{C}$ and $\mathrm{H}$ atoms have been colored light blue or light red based on the parent fragment. The stoppers and part of the thread have been omitted for clarity. 


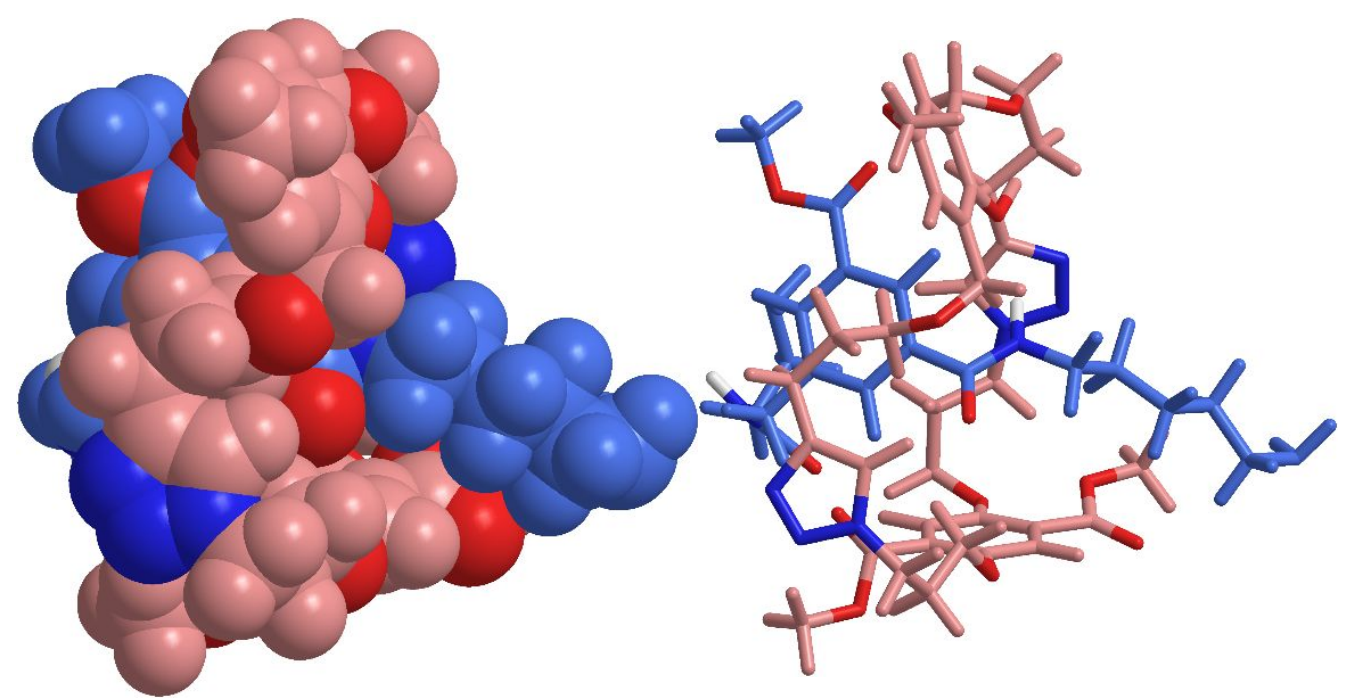

Figure 593. Side view of space filling (left) and tube (right) representations of the calculated structure of [2] rotaxane 3. $\mathrm{C}$ and $\mathrm{H}$ atoms have been colored light blue or light red based on the parent fragment. The stoppers and part of the thread have been omitted for clarity.

XYZ-coordinates of calculated structure of [2]catenane 2

\begin{tabular}{|c|c|c|c|}
\hline & & & \\
\hline & 1.686104 & -1.942203 & 3.05235 \\
\hline & 2.995408 & -1.491741 & 2.83918 \\
\hline & 3.217021 & -0.309339 & 2.11831 \\
\hline & 2.140365 & 0.421098 & 1.59340 \\
\hline & 0.838137 & -0.044069 & 1.79247 \\
\hline & 1.433665 & -3.193447 & 3.86132 \\
\hline & -0.349525 & 0.719729 & 1.28304 \\
\hline & -1.336107 & 0.940867 & 1.97554 \\
\hline & -0.254564 & 1.171767 & -0.02350 \\
\hline & 0.751419 & -4.223341 & 3.05042 \\
\hline & 1.329665 & -5.474508 & 2.83007 \\
\hline & -1.350530 & 1.942910 & -0.66408 \\
\hline & -1.768486 & 1.279194 & -1.98587 \\
\hline & 0.566435 & -6.398022 & 1.89994 \\
\hline & 2.387938 & -5.778905 & 3.36079 \\
\hline & -2.538529 & 2.250743 & -2.88841 \\
\hline & -3.977013 & 2.506701 & -2.41873 \\
\hline & -4.954048 & 1.431595 & -2.91205 \\
\hline & -6.393904 & 7967 & -2.48633 \\
\hline & -7.367518 & 0.597170 & -2.76913 \\
\hline & -7.070162 & -0.647671 & -1.92215 \\
\hline & -8.169512 & -1.706364 & -2.06559 \\
\hline & -0.915088 & -6.544025 & 2.26176 \\
\hline & -1.687245 & -7.327304 & 1.18930 \\
\hline c & -3.186691 & -7.010581 & 1.26660 \\
\hline & -3.985373 & -7.697729 & 0.15100 \\
\hline & -5.392333 & -7.098226 & 0.00692 \\
\hline & -5.359752 & -5.711683 & -0.65243 \\
\hline & -6.718706 & -5.005998 & -0.56251 \\
\hline & -6.708633 & -3.671538 & -1.31991 \\
\hline 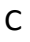 & -8.004244 & -2.882310 & -1.09352 \\
\hline & 4.586067 & 0.214005 & 1.89119 \\
\hline & 4.908633 & 1.105598 & 1.12620 \\
\hline & 5.492184 & -0.429979 & 2.68176 \\
\hline & 6.892480 & -0.046833 & 2.54915 \\
\hline
\end{tabular}




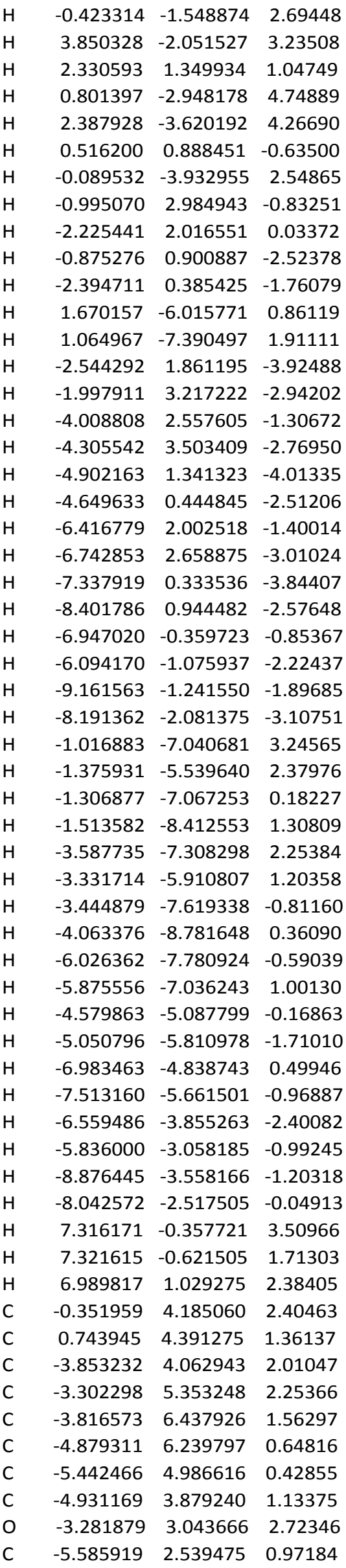




\begin{tabular}{|c|c|c|c|}
\hline & 2.189053 & 33 & \\
\hline & 4.499581 & 1.587442 & 81794 \\
\hline & 4.927523 & 0.198787 & 0 . \\
\hline & 3.672947 & -0.554524 & 0.46697 \\
\hline & 3.871218 & -2.072766 & 847 \\
\hline & 368069 & 0.103212 & -1.60688 \\
\hline & 4.193932 & -1.612247 & 997 \\
\hline & 4.046762 & -0.238511 & 930 \\
\hline & 3759 & 0.614147 & -2.0 \\
\hline & 6.510909 & -1.2 & -1 \\
\hline & 02 & 09 & -1 . \\
\hline & -0.905690 & 300 & 202 \\
\hline & 197 & 4.9 & 1.9 \\
\hline & 3.127270 & 4.989046 & 0.96 \\
\hline & 876 & 3.94 & \\
\hline & 4.727390 & 4.568641 & -0. \\
\hline & 5168 & 5.921872 & \\
\hline & 3.570213 & 6.186020 & 0.36701 \\
\hline & 175 & 3.9 & -1.4 \\
\hline & 6.708786 & 3.0 & -0. \\
\hline & 23 & 2.2 & -1. \\
\hline & 8918 & 0.82 & -1 . \\
\hline & 7785 & -2.7 & -0 . \\
\hline & 5204 & -2.8 & -1.4 \\
\hline & 7777 & -3.4 & -1 \\
\hline & 975 & -3.7 & \\
\hline & 6439 & -3.3 & 494 \\
\hline & 68 & -3.7 & 921 \\
\hline & 2980 & -2.6 & 93 \\
\hline & 46 & -2.5 & \\
\hline & 5887 & -2.5 & -2.2 \\
\hline & 085 & 03 & 89 \\
\hline & 45 & 762 & -0.5 \\
\hline c & 651 & 0.38 & -2.6 \\
\hline & 2755 & 38 & -2.2 \\
\hline $\mathrm{O}$ & 3.029268 & 925 & -3.72369 \\
\hline & 483 & 1.95 & -4.2 \\
\hline & 537 & -1.8 & $-0 . \varepsilon$ \\
\hline & 8.279522 & -1.7 & 174 \\
\hline c & 689 & -2.5 & -1.8 \\
\hline & 324 & 121 & 568 \\
\hline & 49 & 00 & \\
\hline & 512 & 14 & 16 \\
\hline & & 3.4 & 0.8 \\
\hline & 0.374704 & 5.10 & 0.59548 \\
\hline & 691 & 239 & 1.69692 \\
\hline $\mathrm{H}$ & 932 & 580 & -0.26689 \\
\hline & -3.400469 & 246 & 2.26287 \\
\hline $\mathrm{H}$ & -6.217433 & 2.472261 & 0.05513 \\
\hline & -6.186233 & 2.278916 & 82 \\
\hline $\mathrm{r}$ & -2.204522 & 6.538332 & 3.71525 \\
\hline & -2.238907 & 4.759369 & 4.05195 \\
\hline 11 & -5.230857 & -0.019531 & 1.93094 \\
\hline & -5.783191 & 0.037178 & 0.20343 \\
\hline 11 & -3.341586 & -0.184415 & -0.53293 \\
\hline & 045 & -0.284872 & 1.16098 \\
\hline 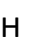 & -4.080826 & -2.445893 & 1.47567 \\
\hline $\mathrm{H}$ & -4.766591 & -2.356474 & -0.1649 \\
\hline
\end{tabular}




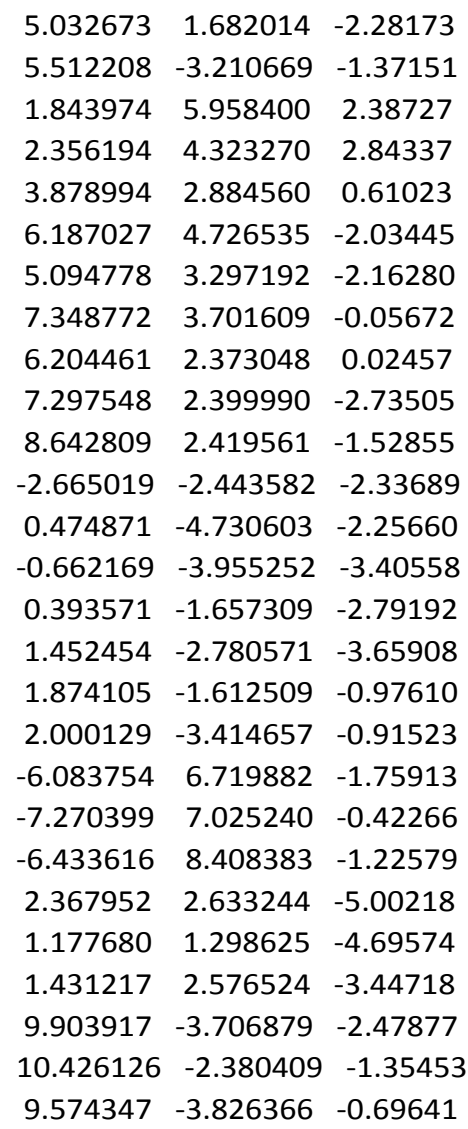

XYZ-coordinates of calculated structure of [2]rotaxane 3

$\begin{array}{llll}C & 2.784577 & -6.791147 & -4.84476 \\ C & 3.372452 & -5.563109 & -5.55303 \\ C & 2.823995 & -3.597213 & -0.61402 \\ C & 0.602196 & -2.031357 & -1.22077 \\ C & 1.446141 & -2.461580 & -2.25367 \\ C & 2.564013 & -3.243094 & -1.94631 \\ C & 1.984494 & -3.158320 & 0.42218 \\ C & 0.862316 & -2.386236 & 0.11046 \\ C & -0.109239 & -1.865575 & 1.13255 \\ C & 1.120050 & -2.078829 & -3.67876 \\ C & -0.170594 & -4.035917 & -4.55468 \\ C & -0.073247 & -5.376067 & -5.26073 \\ C & 0.287698 & -6.488341 & -4.26903 \\ C & 1.738448 & -6.462621 & -3.76978 \\ C & 4.438707 & -4.839878 & -4.71991 \\ O & -1.210981 & -3.629938 & -4.05153 \\ N & 0.996373 & -3.275747 & -4.54142 \\ C & 4.844209 & -3.503778 & -5.35884 \\ C & 6.247717 & -3.040387 & -4.94696 \\ \text { N } & -0.340307 & -2.652349 & 2.24400 \\ O & -0.698749 & -0.799144 & 0.96631 \\ C & -1.353211 & -2.260613 & 3.25923 \\ C & -2.311147 & -3.428565 & 3.53271 \\ C & -3.559189 & -2.947910 & 4.28065 \\ C & -4.453624 & -4.122934 & 4.70480 \\ C & -5.903729 & -3.663498 & 4.88552 \\ C & -6.825177 & -4.779542 & 5.39320 \\ C & -8.310259 & -4.402651 & 5.27204 \\ C & -8.842650 & -3.532307 & 6.41929\end{array}$




\begin{tabular}{|c|c|c|c|}
\hline & 520 & -2.10 & \\
\hline & 8.616025 & -1.328974 & 20340 \\
\hline & 7.707942 & -0.653564 & \\
\hline & 8.064280 & 0.174300 & 3.30781 \\
\hline & 9734 & -0.3 & 9427 \\
\hline & 8.676515 & 1.233178 & 3.3781 \\
\hline & -7.848375 & 0.391770 & 0.81983 \\
\hline & -6.998747 & 1.672442 & 0.7 \\
\hline & -6.418021 & 3.768481 & -0.53825 \\
\hline & -6.032515 & 4.296066 & -1.7 \\
\hline & -5.167016 & 5.388261 & -1.85539 \\
\hline & -4.659837 & 5.993185 & -0.6 \\
\hline & -5.062381 & 5.478230 & 0.54611 \\
\hline & -5.928246 & 4.384563 & 0.6 \\
\hline & -7.410380 & 1.727426 & -1.6 \\
\hline & -6.182891 & 1.287003 & -2.2 \\
\hline & -6.1 & 0.44 & -3.3 \\
\hline & -7.328827 & 0.000670 & -3.9 \\
\hline & 118 & 0.42 & \\
\hline & -8.590542 & 1.270737 & -2.28457 \\
\hline & 917 & $2.60 €$ & -0.4 \\
\hline & -8.767190 & 3.294345 & -0.21297 \\
\hline & 014 & 4.073 & -1.24762 \\
\hline & 10.527049 & 4.742368 & -1.0804 \\
\hline & 1.233666 & 4.65 & 0.1279 \\
\hline & 0.673703 & 3.8 & 1.1 \\
\hline & 9370 & 3.22 & 1.0 \\
\hline & 7847 & -2.72 & -3.4 \\
\hline & 7.383739 & -1.968482 & -3.0 \\
\hline & 7.569471 & -1.733171 & -1.5 \\
\hline & 7.760113 & -0.404220 & -1.2 \\
\hline & & -2.6 & \\
\hline & 05 & 0.00 & 0.1 \\
\hline & 7.0 & 1.05 & 0.7 \\
\hline & 56 & 3.4 & 0.9 \\
\hline C & 70 & 4.6 & \\
\hline & & 5.4 & 0.7 \\
\hline & 4.239234 & 5.14 & 1.90322 \\
\hline & 69 & 4.00 & 2.6 \\
\hline & 5.664657 & 3.184 & 2.16193 \\
\hline & 8.681544 & 2.977995 & 1.24325 \\
\hline & .227738 & 4.253978 & 1.00467 \\
\hline & 162 & 4.700801 & 1.71788 \\
\hline & 617 & 3.892246 & 2.69850 \\
\hline & 870 & 2.635809 & 2.95077 \\
\hline & 761 & 2.185403 & 2.23607 \\
\hline & .444199 & 2.540858 & 0.4 \\
\hline & 220 & 2.663056 & -1.0 \\
\hline C & .528716 & 2.638429 & -1.91474 \\
\hline & 948 & 2.673820 & -3.29792 \\
\hline c & 7.969750 & 2.731373 & -3.87710 \\
\hline & 9.078905 & 2.737761 & -3.02269 \\
\hline C & 8.920105 & 2.703722 & -1.63326 \\
\hline & 3.991419 & -4.442731 & -0.26803 \\
\hline $\mathrm{O}$ & 4.230282 & -4.975322 & 0.79672 \\
\hline & 4.805989 & -4.621632 & -1.3598 \\
\hline C & 6.077837 & -5.299795 & -1.1288 \\
\hline C & -3.752848 & 7.220646 & -0.8024 \\
\hline
\end{tabular}




\begin{tabular}{|c|c|c|c|}
\hline & 24 & 22 & \\
\hline & 2.931619 & 946 & 0.48357 \\
\hline & -4.656440 & 8.445833 & 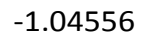 \\
\hline & -7.239029 & -0.945820 & -5.13538 \\
\hline & -6.752931 & -2.317215 & 2257 \\
\hline & -6.235178 & -0.382186 & -6.16326 \\
\hline & -8.596971 & -1.127097 & 103 \\
\hline & -12.554615 & 5.394782 & 0.35350 \\
\hline 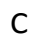 & 13.199217 & 5.847462 & -0 \\
\hline & 12.257549 & 6.634664 & 158 \\
\hline 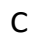 & 376 & 776 & 7980 \\
\hline & 3.052697 & 5.97 & 32 \\
\hline & 1.7 & 94 & \\
\hline & 2.988653 & 7.354454 & 267 \\
\hline & 181 & 6.19 & 753 \\
\hline & 12.151668 & 4.423995 & 3.47018 \\
\hline C & 098 & 5.019746 & 158 \\
\hline 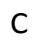 & 12.856628 & 3.319326 & 4.28054 \\
\hline C & 47 & 34 & 700 \\
\hline C & 370 & 2.73 & -5.4 \\
\hline c & & 3.6 & \\
\hline C & 7.9 & 1.275 & 274 \\
\hline c & 9.4 & 3.25 & 97 \\
\hline 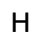 & 35 & -7.46 & -5 . \\
\hline $\mathrm{H}$ & 375 & -7.37 & 252 \\
\hline $\mathrm{H}$ & & -4.8 & 061 \\
\hline $\mathrm{H}$ & 02 & -5.8 & 670 \\
\hline 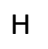 & & 42 & 794 \\
\hline $\mathrm{H}$ & 07 & -3.5 & -2 \\
\hline $\mathrm{H}$ & & 56 & \\
\hline $\mathrm{H}$ & 73 & -1.4 & -3.7 \\
\hline $\mathrm{H}$ & 7 & -1.4 & \\
\hline $\mathrm{H}$ & 32 & -5.5 & -5 \\
\hline $\mathrm{H}$ & 52 & -5.35 & -6 \\
\hline $\mathrm{H}$ & 690 & -6.4 & -3 \\
\hline $\mathrm{H}$ & 217 & -7.468247 & 366 \\
\hline $\mathrm{H}$ & 038 & -5.487625 & 743 \\
\hline 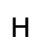 & 22 & -7.203322 & 1782 \\
\hline$\cdots$ & 4.078000 & -4.6 & 872 \\
\hline 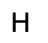 & 15 & -5.4 & 632 \\
\hline $\mathrm{H}$ & 43 & -3.6 & 879 \\
\hline $\mathrm{H}$ & 24 & -3.5 & -6 \\
\hline $\mathrm{H}$ & & & 590 \\
\hline $\mathrm{H}$ & & -3.82 & -5.2 \\
\hline $\mathrm{H}$ & 6.532249 & -2.160662 & -5.56431 \\
\hline $\mathrm{H}$ & & -3.5 & 2.4 \\
\hline $\mathrm{H}$ & -0.829115 & -1.95 & 4.19210 \\
\hline $\mathrm{H}$ & 9763 & -1.364744 & 2.89020 \\
\hline $\mathrm{H}$ & 071 & -3.911287 & 2.57998 \\
\hline $\mathrm{H}$ & & -4.2 & 17 \\
\hline . & -3.27 & -2.36 & 5.17355 \\
\hline 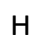 & -4.129958 & -2.252424 & 194 \\
\hline $\mathrm{H}$ & -4.413696 & -4.932268 & 3.93842 \\
\hline $\mathrm{H}$ & -4.066255 & -4.578350 & 5.63461 \\
\hline 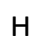 & -6.289372 & -3.273256 & 3.91644 \\
\hline $\mathrm{H}$ & & -2.806223 & 5.59145 \\
\hline $\mathrm{H}$ & -6.576994 & -5.027741 & 6.4422 \\
\hline $\mathrm{H}$ & -6.642748 & -5.706899 & 4.81652 \\
\hline
\end{tabular}




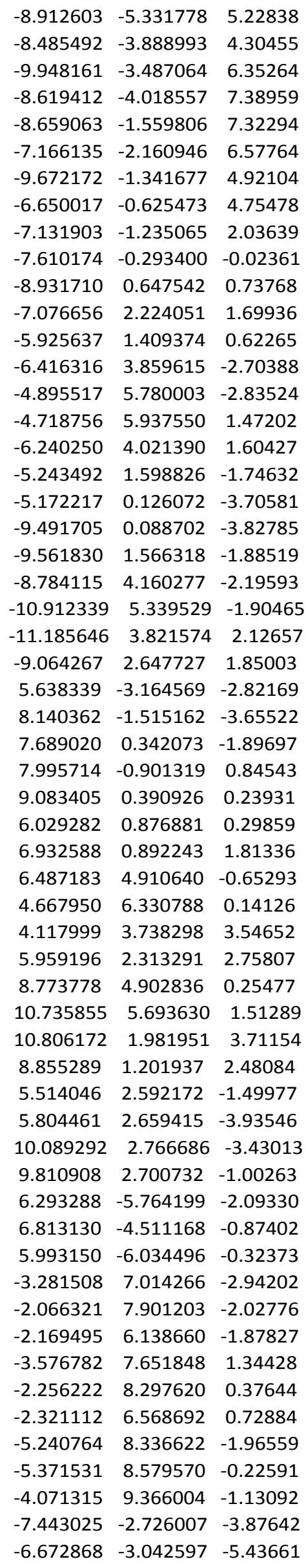




\begin{tabular}{|c|c|c|c|}
\hline & 3 & -2.2 & \\
\hline & -6.524825 & 0.624387 & -6.48257 \\
\hline & -6.182294 & -1.013503 & -7.05486 \\
\hline & -5.223693 & -0.316735 & -5.74830 \\
\hline & -9.342545 & -1.575527 & -5.17612 \\
\hline & -8.993665 & -0.169911 & -6.19565 \\
\hline & -8.504008 & -1.784969 & -6.71102 \\
\hline & -13.384865 & 4.995617 & -1.63433 \\
\hline & -14.160064 & 6.340713 & -0.79352 \\
\hline & -12.565184 & 6.560374 & -1.50880 \\
\hline & -11.8 & 128 & 2.18763 \\
\hline & -13.165423 & 7.210867 & 1.42014 \\
\hline & -11.539430 & 748 & 883 \\
\hline & -14.527820 & 4.959069 & 1.20365 \\
\hline & -13.714367 & 238 & 0.51949 \\
\hline & -13.202335 & 4.194306 & 2.07861 \\
\hline & 1.768922 & 4.200667 & 2.55008 \\
\hline & 0.876341 & 5.719724 & 2.40190 \\
\hline & 1.67 & 5.0 & 0.98775 \\
\hline & 3.903865 & 7.92 & 1.88774 \\
\hline & 2.15 & 7.9 & 2.0 \\
\hline & 2.846111 & 7.267334 & 0.63017 \\
\hline & 2.357271 & 6.84 & 4.28122 \\
\hline & 3.091036 & 5.25 & 4.47474 \\
\hline & 4.112884 & 6.66 & 091 \\
\hline & 981 & 41 & 629 \\
\hline & 14.079766 & 459 & 966 \\
\hline & 13.4 & & 194 \\
\hline & 12.199966 & 706 & 5.04504 \\
\hline & 13.7 & 92 & 19 \\
\hline & 13.198474 & 292 & 3.63357 \\
\hline & 12.478040 & 5.93 & 5.02280 \\
\hline & 11.177407 & 841 & 3.90115 \\
\hline & 10.915401 & 640 & 5.14026 \\
\hline & 7.082324 & 4.64 & -5.64686 \\
\hline & 7.167212 & 3.685655 & -7.12838 \\
\hline & 6.027960 & 3.244527 & -5.85910 \\
\hline & 8.717757 & 0.623785 & -5.44888 \\
\hline & 8.037827 & 1.207735 & -6.97185 \\
\hline & 6.974452 & 0.870992 & -5.60212 \\
\hline & 9.576828 & 3.29 & -6.93178 \\
\hline & 9.672232 & 4.275102 & -5.46688 \\
\hline & 10.315079 & 2.625616 & -5.48035 \\
\hline & 2.554360 & 0.384563 & 1.03247 \\
\hline & 3.409355 & 1.447040 & 0.34459 \\
\hline & 3.744373 & -2.25 & 3.65830 \\
\hline & 3.641469 & -0.855687 & 3.60133 \\
\hline & 4.801927 & -0.088368 & 3.73449 \\
\hline 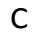 & 6.030011 & -0.730552 & 3.92398 \\
\hline & 6.141323 & -2.129057 & 3.96773 \\
\hline 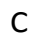 & 4.990100 & -2.906273 & 3.82001 \\
\hline & 2.572568 & -2.968853 & 3.52635 \\
\hline 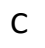 & 5.100713 & -4.402866 & 3.78329 \\
\hline 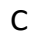 & 2.314735 & -0.175725 & 3.38972 \\
\hline 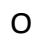 & 3.970672 & -4.915658 & 4.54955 \\
\hline 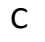 & 3.694586 & -6.328861 & 4.34298 \\
\hline C & 2.244524 & -6.461415 & 3.88513 \\
\hline & 2.057263 & -6.038734 & 2.42445 \\
\hline
\end{tabular}




\begin{tabular}{|c|c|c|c|}
\hline & 38 & 91 & \\
\hline & 3.927913 & -3.051380 & -1.18859 \\
\hline & 4.578597 & -2.575986 & 2567 \\
\hline & 4.461908 & -1.225251 & 30596 \\
\hline & 3.072786 & -0.833979 & -1.66710 \\
\hline & 3.176459 & -2.189641 & -1.99854 \\
\hline & 2.419502 & 0.863486 & 2.40112 \\
\hline & 3.040136 & 1.658308 & -1.12579 \\
\hline & 1.758468 & 2.382280 & -1.31074 \\
\hline & 0.757725 & 2.709810 & -0.3 \\
\hline & -0.2 & 57 & 2987 \\
\hline & 0.181415 & 5311 & 862 \\
\hline & 43 & 769 & -2 \\
\hline & -1.503146 & 3.914231 & -0.63894 \\
\hline & -2.635878 & 700 & $-0 . \varepsilon$ \\
\hline & -2.471310 & 1.662885 & 0.04801 \\
\hline & 4611 & 320 & -0 \\
\hline & 0.651418 & -6.136974 & 1.96481 \\
\hline & 235 & -6.0 & \\
\hline & -1.238333 & -6.1 & 325 \\
\hline & -1.5 & -6.3 & \\
\hline & -0.446686 & -6.3 & 798 \\
\hline & 731 & -6.1 & -0. \\
\hline & -1.918447 & -5.3 & -1 \\
\hline & -3.170992 & -5.07 & -2 \\
\hline & -4.146483 & -4.3 & \\
\hline c & 7.243364 & -0.0 & 4.0 \\
\hline & & & \\
\hline & -5.371166 & -3.4 & $0 . \varepsilon$ \\
\hline & & & \\
\hline & -5.00 & -4.7 & 0.8 \\
\hline & -5.64 & -5.6 & 35 \\
\hline & -2.263326 & 0.00 & -2.5 \\
\hline $\mathrm{O}$ & -1.061332 & -0.0 & -2 \\
\hline & -3.073710 & 0.823944 & -3 \\
\hline c & -2.419431 & 1.646708 & -4.3 \\
\hline & 3.026807 & -0.6 & \\
\hline & 1.525982 & 166 & 0.6 \\
\hline & 3.318685 & 552 & 0.9 \\
\hline & 204 & 1.1 & 0.4 \\
\hline & 79 & 05 & \\
\hline & 59 & -2.5 & 80 \\
\hline & 685 & 317 & 10 \\
\hline & 277 & -4.7 & 4.27092 \\
\hline & 5.044562 & -4.773751 & 2.73527 \\
\hline & 1.516289 & -0.889726 & 3.11394 \\
\hline $\mathrm{H}$ & 2.003796 & 0.393424 & 859 \\
\hline & 4.407198 & -6.775968 & 189 \\
\hline $\mathrm{H}$ & .846697 & -6.770051 & 5.34662 \\
\hline & .899495 & -7.5 & \\
\hline H & 1.583566 & -5.856889 & 4.54 \\
\hline & 2.426637 & -4.986317 & 2.28815 \\
\hline 11 & 2.715720 & -6.643932 & 1.76079 \\
\hline & -4.951812 & -0.819937 & 1.19868 \\
\hline 11 & -2.666769 & -2.577610 & -2.89391 \\
\hline & 43 & 2.227919 & -1.62693 \\
\hline 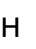 & 2.988660 & 0.682955 & -1.65864 \\
\hline $\mathrm{H}$ & 0.691241 & 2.506895 & 0.6701 \\
\hline
\end{tabular}




$\begin{array}{rrr}-1.751407 & 4.858818 & -1.20841 \\ -1.390543 & 4.203582 & 0.43083 \\ -3.609311 & 3.396547 & -0.57544 \\ -2.740416 & 2.613803 & -1.88728 \\ -1.613567 & 1.019331 & -0.22236 \\ -2.421317 & 1.890773 & 1.12875 \\ 0.651893 & -5.884899 & -0.27514 \\ -2.520995 & -7.253879 & -0.51932 \\ -3.235923 & -5.769970 & 0.21120 \\ -1.174617 & -5.904913 & -2.12248 \\ -1.430900 & -4.419791 & -1.18670 \\ -2.936102 & -4.480807 & -3.21165 \\ -3.748651 & -5.976204 & -2.57305 \\ 6.741188 & 1.970509 & 4.16182 \\ 6.718367 & 1.046903 & 5.72466 \\ 8.287976 & 1.394542 & 4.90155 \\ -6.698815 & -5.403252 & 1.88021 \\ -5.515435 & -6.634276 & 1.26217 \\ -5.092815 & -5.589171 & 2.69811 \\ -1.489582 & 2.083827 & -3.95641 \\ -2.216033 & 1.019339 & -5.22015 \\ -3.175519 & 2.410945 & -4.55770\end{array}$




\section{References}

1. A. Pegoli, X. She, D. Wifling, H. Hübner, G. Bernhardt, P. Gmeiner, \& M. Keller, J. Med. Chem., 2017, 60, 3314.

2. F. Miege, C. Meyer, \& J. Cossy, Angew. Chem. Int. Ed., 2011, 50, 5932.

3. C.J. Galvin, E.D. Bain, A. Henke, \& J. Genzer, Macromolecules, 2015, 48, 5677.

4. A.M. Ako, M.S. Alam, S. Mameri, Y. Lan, M. Hibert, M. Stocker, P. Müller, C.E. Anson, \& A.K. Powell, Eur. J. Inorg. Chem., 2012, 2012, 4131.

5. Q.H. Sodji, V. Patil, J.R. Kornacki, M. Mrksich, \& A.K. Oyelere, J. Med. Chem., 2013, 56, 9969.

6. L. Steemers, M.J. Wanner, A.W. Ehlers, H. Hiemstra, \& J.H. van Maarseveen, Org. Lett., 2017, 19, 2342.

7. L. Steemers, M.J. Wanner, M. Lutz, H. Hiemstra, \& J.H. van Maarseveen, Nat. Commun., 2017, 8, 15392.

8. J.J.P. Stewart, J Mol Model, 2007, 13, 1173.

9. M.J. Frisch, G.W. Trucks, J.R. Cheeseman, G. Scalmani, M. Caricato, H.P. Hratchian, X. Li, V. Barone, J. Bloino, G. Zheng, T. Vreven, J.A. Montgomery, G.A. Petersson, G.E. Scuseria, H.B. Schlegel, H. Nakatsuji, A.F. Izmaylov, R.L. Martin, J.L. Sonnenberg, J.E. Peralta, J.J. Heyd, E. Brothers, F. Ogliaro, M. Bearpark, M.A. Robb, B. Mennucci, K.N. Kudin, V.N. Staroverov, R. Kobayashi, J. Normand, A. Rendell, R. Gomperts, V.G. Zakrzewski, M. Hada, M. Ehara, K. Toyota, R. Fukuda, J. Hasegawa, M. Ishida, T. Nakajima, Y. Honda, O. Kitao, \& H. Nakai, Gaussian 09. 